TEXAS TECH UNIVERSITY

3 1295011949798 
$A m G-1522$

DEPARTMENT OF THE INTERIOR

Albert B. FALL, Secretary

United States Geological Survey George OTIS SMith, Director

Bulletin 728

\title{
THE OCCURRENCE AND USES OF PEAT IN THE UNITED STATES
}

BY

E. K. SOPER AND C. C. OSBON

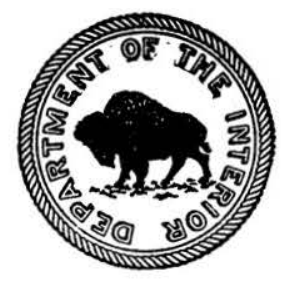

LIBRARY

TEXAS TECHNOLOGICAL COLLEGE

LUBBOCK, TEXAS

\author{
WASHINGTON \\ GOVERNMENT PRINTING OFFIOE \\ 1922
}


ADDITIONAL COPIES

OF THIS PUBLICATION MAY BE PROCURED FROM THE SUPERINTENDENT OF DOCUMENTS GOVERNMENT PRINTING OFFICE WASHINGTON, D. c.

$\Delta T$

50 CENTS PER COPY 


\section{CONTENTS.}




Properties of peat-Continued. $\quad$ Page.

Chemical composition-Continued.

Analyses-Continued.

Indiana _- 27

Iowa -

Maine_-_- 30

Massachusetts _. 34

Michigan _- 35

Minnesota _-_._- 37

New Hampshire

New Jersey

New York _. 45

Ohio _- 48

Pennsylvania___- 49

Rhode Island _. 49

Vermont_._. 49

Virginì and North Caroliva $\quad 50$

Wisconsin _. 51 .

Conclusions

Uses of peat._- 59

Agricultural uses _.. 59

Fertilizer -

General features _._. 59

Methods of preparation. 61

Peat soils - 62

General features 62

Soil treatment_____ 64

Stock feed _- 66

Absorbent and disinfectant___ 66

Fuel_- 66

Geñeral features.______ 66

Peat fuel in the United States___. 67

Mëthods of preparation 69

Cut peat._- 69

Machine peat__._. 69

Powdered peat_-_- 71

Peat briquets____ 71

Peat coke and charcoal____- 72

Producer-gas and by-products.____- 72

Peat and peat moss as antiseptics and medicaments

Antiseptic dressing - 73

Mud baths 73

Other uses_-_- 74

Packing material _._- 74

Paper, wood, and cloth_. 74

The peat industry in the United States

Production___- 74

Imports and exports_._____

Summary

Consumption____ 75

Distribution of peat plants_-_- 75

Producers of peat in the United States_- 76 
Page.

Commercial factors

Causes of failure-_. 76

Location _- 76

Quantity and quality

Size of deposit____ 77

Selection of samples. 78

Simple tests___ 78

Preparation of surface._. 79

Processes and machinery

Markets _- 80

Areas of sphagnum moss

Northwestern States________ 80

Northeastern States_______ 80

Other States______- 81

Marl and other limestone deposits associated with beds of peat_________ 82

General conditions_-_____ 82

Distribution of limestone by States____ 82

New England States___ 82

New York, New Jersey, and Pennsylvania_____ 82

Maryland, Delaware, Virginia, West Virginia, North Carolina, and South Carolina.

Tennessee, Mississippi, Alabama, Georgia, and Florida_______ 84

Wisconsin and Michigan._. 85

Illinois, Indiana, Ohio, and Kentucky 86

Minnesota _- 88

Iowa -

Louisiana _._._._- 91

Distribution of peat deposits and quantity of peat available_____-_ 91

General features_____ 91

Northern region

Minnesota _- 93

Wisconsin _- 94

Michigan _- 96

General features____- 96

Dickinson County 97

Eaton County

Houghton County

Iron County

Kalamazoo County-_-_. 99

Lapeer County

Luce County_-___- 100

Marquette County 100

Mecosta County

Muskegon County____ 101

Oakland County 101

St. Clair County

Shiawassee County

Washtenaw County 102

Iowa -

Illinois _- 104

General features_____- 104

Kane County 
Distribution of peat deposits and quantity of peat available-Continued. Page. Northern region-Continued.

Illinois-Continued.

Kankakee County

Lake County-_-_._- 105

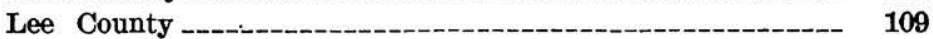

Mason and Tazewell counties_____- 109

Whiteside County_-_- 110

Indiana -._-_-_.-_ 112

General features_-_- 112

Allen County -_-_--_-_-_-_-_-_ 113

Dekalb County _._- 113

Elkhart County

Fulton County-_-_-_-_- 115

Jasper County_-_-_-_-_-_-_- 116

Kosciusko County

Marshall County

Noble County

Pulaski County

St. Joseph County

Starke County_-_-_._- 119

Steuben County

Ohio - -

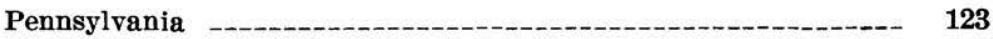

Columbia County _..-_- 123

Crawford County _._.

Philadelphia County _......-_ 124

Other counties_._-_.

New York ${ }^{i}$ Ot_.

General features_-_- 125

Cayuga County_-_-_-_-_-_- 126

Clinton County -.-_-_- 126

Dutchess County

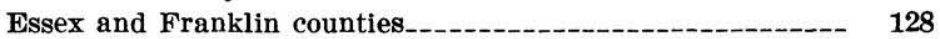

Genesee and Orleans counties_._- 133

Herkimer County-_-_-_-_-_- 135

Onondaga County

Orange County -_-_-_- 138

St. Lawrence County_-_-_-_-_-_-_-_-_-_-_-_-_-_-_- 140

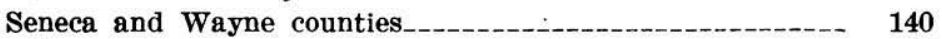

New Jersey _-_-_. 143

General features_-_-_- 143

Sussex County _._-

Warren County

Maine -_-_-_-_-_-_-_- 146

New Hampshire -...._- 150

General features_-_-_._- 150

Cheshire County

Hillsborough County

Rockingham County-_-_-_-_- 154

Other counties_._. 156

Vermont _.-.- 156

General features_. 
Distribution of peat deposits and quantity of peat available-Continued. Page. Northern region-Continued.

Vermont-Continued.

Addison County

Chittenden County 159

Franklin County_____ 160

Grand Isle County-____- 161

Rutland County

Massachusetts _._-___-_-___-_ 165

General features__-_._- 165

Berkshire County

Essex and Suffolk counties_._. 166

Middlesex County

Norfolk County _-_- 177

Plymouth County -..- 179

Worcester County-_-_ 181

Connecticut_-_-_-_-_-_-_-_-_-_- 183

General features_-_-_- 183

Fairfleld County -_-___- 184

Hartford County

Middlesex County

New Haven County

Rhode Island _. 188

Bristol County

Providence County -_-_-_-_-_ 189

Washington County _.._- 190

Other States__-_-_-_ 191

Atlantic coastal region

Virginia and North Carolina________-_ 191

General features_-_-_ 191

Arlington and Fairfax counties, Va

The Dismal Swamp district_-__- 192

Geography and geology

Flora_-___-_-_ 193

Properties_-_-_-_-_-_-_-_-_- 195

Associated marl_-_-_-_-_- 196

Distribution and quantity

Areas tested _.____ 197

Norfolk County, Va

Nansemond County, Va___________-_ 198

Currituck County, N. C_-__-_-_-_ 198

Camden County, N. C_-__- 199

Other counties_-__-_- 199

South Carolina_-__- 199

Georgia _-____-__-_- 199

Florida_-_-_-_- 199

General features_- 199

West coast region

West pine-hill region.-.- 200

West limestone region_-_-_- 200

Middle hammock region______- 200

Lime-sink region_-_- 200

Middle flatwood region. 200

Gulf hammock region.-_- 200 
Distribution of peat deposits and quantity of peat available-Continued. Page.

Atlantic coastal region-Continued.

Florida-Continued.

Lake region _-_-_-_-_-_- 200

East flatwood region

East coast region-_-_- 200

South flatwood region._-_. 201

Miami limestone region__-_-_-_._- 201

Coast prairie region_-_- 201

Key region _-_. 201

Other States_-_-_-_ 201

Other regions_._-_-_. 201

Gulf coast__._-_. 201

California _._-_._- 201

Oregon and Washington

Index _-_-_-_-_-_-_ 203

\section{ILLUSTRATIONS.}

Plate I. Map of the United States showing distribution of peat deposits and approximate location of plants that produced peat in 1918 In pocket.

II. Types of mature peat deposits: $A$, Filled-basin peat deposit; $B$, Built-up peat deposit formed on a relatively flat surface--

III. $A, B$, Peat-forming basins.

IV. Commercial products of peat: $A$, Excellent stand of corn raised upon reclaimed peat land at Big Island, Orange County, N. Y.; $B$, Dry hand-cut peat fuel and slane with which excavated.

v. Predominant types of peat deposits in northern Minnesota: $A$, Spruce-sphagnum bog; $B$, Tamarack swamp

VI. Map showing distribution of peat in northern Minnesota

VII. Map of Wisconsin showing distribution of undrained land.-.-.-

VIII. Map showing distribution of peat in the eastern part of the northern peninsula of Michigan.

IX. Map showing distribution of peat and muck in Lake County, Ill_-

$\mathrm{X}$. Map showing distribution of peat in Ohio

XI. The winning of a peat swamp: $A$, Native section of Cicero Swamp, Onondaga County, N. Y.; B, Reclaimed peat swamp in the Wallkill River valley near Big Island, Orange County, N. Y.

XII. Map showing distribution of peat in northern New Jersey

XIII. Map of Maine showing location of peat deposits

XIV. The evolution of a peat bog: A, Bristol Pond, Addison County, Vt.; $B$, Spruce-sphagnum bog on the shore of Bristol Pond.-

XV. $A$, Cross section of composite peat bed near New Haven, Conn.; $B$, Typical peat meadow near Lowell, Mass

XVI. Map of southeastern Virginia and northeastern North Carolina showing distribution of peat and location of test borings in the Dismal Swamp

XVII. Black gum and bald cypress plant association in the Dismal Swamp, Va

XVIII. Map of Florida showing regions containing peat deposits_-_--- 
Fraure 1. Diagram showing relation between the weight and water content of a ton of raw peat at different stages of evaporation.-

Page.

2. Map of Iowa showing area covered by the Wisconsin glacial drift and approximate location of deposits that have been tested for peat

3. Map of Cattail Valley, Whiteside County, Ill

4. Map of Wickham Marsh, Essex County, N. Y., showing approximate position of test borings for peat

5. Map of peat deposit southwest of Bloomingdale station, Essex and Franklin counties, N. Y., showing approximate position of test borings for peat

6. Map of peat bog south of Lake Clear, Franklin County, N. Y., showing approximate position of test borings for peat.--.-

7. Map of peat deposit northwest of Tupper Lake, Franklin County, N. Y., showing approximate position of test borings for peat-

8. Map of the eastern part of Oak Orchard Swamp, Genesee and Orleans counties, N. Y., showing approximate position of test borings for peat.

9. Map of part of Cicero Swamp, Onondaga County, N. Y., showing approximate position of test borings for peat.

10. Map of the "drowned lands" of Wallkill River valley, Orange County, N. Y., showing approximate position of test borings for peat.

11. Map of the northeastern part of Montezuma Marsh, Seneca and Wayne counties, N. Y., showing approximate position of test borings for peat

12. Map of the northwestern part of Montezuma Marsh, Seneca and Wayne counties, N. Y., showing approximate position of test borings for peat.

13. Map of peat deposit in Byram Township, Sussex County, N. J., showing approximate position of test borings for peat

14. Map of peat deposit along Pequest River, Warren County, N. $\mathbf{J}$

15. Map of peat deposits near East Jaffrey, Cheshire County, N. H., showing approximate position of test borings for peat

16. Map of peat deposits near Ponemah, Hillsborough County, N. H., showing approximate position of test borings for peat

17. Map of Spruce Swamp, Rockingham County, N. H., showing approximate position of test borings for peat

18. Map of peat bogs near Bristol Pond and Pond Brook, Addison County, vt., showing approximate position of test borings for peat.

19. Map of peat deposit southwest of Porter Point, Chittenden County, vt., showing approximate position of test borings for peat

20. Map showing swamps near South Hero and Grand Isle station, Grand Isle County, Vt

21. Map of bog near Isle La Motte station, Grand Isle County, Vt., showing approximate position of test borings for peat 
Frgure 22. Map of swamp and marsh near Alburg and Kelly Bay, Grand Isle County, Vt., showing approximate position of test borings for peat.

23. Map of salt marshes near Revere Beach, Essex and Suffolk counties, Mass., showing approximate position of test borings for peat.

Page.

24. Map of peat deposit near Mystic Pond, Essex County, Mass., showing approximate position of test borings for peat

25. Map of peat deposits near Littleton and North Littleton stations, Middlesex County, Mass., showing approximate position of test borings for peat.

26. Map of Tophet Swamp and adjoining bog, Middlesex County, Mass., showing approximate position of test borings for peat

27. Map of peat deposit near East Lexington, Middlesex County, Mass., showing approximate position of test borings for peat

28. Map of marshes adjoining Concord River, Middlesex County, Mass., showing approximate position of test borings for peat

29. Map of peat bog near Norwood, Norfolk County, Mass., showing approximate position of test borings for peat

30. Map of marsh adjoining Charles River west of Boston, Mass., showing approximate position of test borings for peat

31. Map of Great Swamp and marsh adjoining Quaboag River, Worcester County, Mass., showing approximate position of test borings for peat

32. Map of salt marsh adjoining Quinnipiac River, New Haven County, Conn 


\title{
THE OCCURRENCE AND USES OF PEAT IN THE UNITED STATES.
}

\author{
By E. K. Soper and C. C. Osbon. \\ INTRODUCTION. \\ SCOPE AND PURPOSE OF THE REPORT.
}

This report, which contains the results of studies undertaken during the World War, describes the peat deposits of the region lying east of the ninety-seventh meridian and north of an irregular line drawn eastward through the northern parts of Iowa, Illinois, Indiana, Ohio, Pennsylvania, and New Jersey, including approximately the area covered by the Wisconsin or last glacial drift; a relatively narrow strip of land extending 25 to 50 miles inland on the Atlantic coast from New Jersey to southern Florida and along the Gulf coast to the Mexican boundary; and small scattered areas in the Pacific Coast States. (See Pl. I.) These regions include practically all the valuable peat deposits in this country and nearly all the swamp land except the bayous and lowlands along the Mississippi, where peat can not form because the water contains too much sediment and the heavy rainfall is unevenly distributed throughout the year.

It has been known for many years that the United States contains large deposits of peat, but little detailed information concerning the quantity and quality of this peat or the uses for which it is best adapted has heretofore been available except some reports on the peat deposits of Maine and a few other States. This report is intended to show the method of formation, distribution, quantity, and quality of the peat in the United States, to indicate the uses for which it is best suited, to point out the possibilities offered by the commercial utilization of peat, and to serve as a guide for future investigations. It contains conclusions based on a study of the origin, occurrence, and distribution of peat in the areas considered, a general account of the uses of peat and peat moss for fertilizer, fuel, surgical dressings, and other purposes, and descriptions of the methods of measuring and testing peat deposits and of the processes of manufac- 
turing peat products; but as complete descriptions of machinery and manufacturing methods are given in other publications, references to which will be found in this report, the technical features of the work of peat production are treated only briefly.

\section{PERSONNEL AND ACKNOWLEDGMENTS.}

The geologic field work in Minnesota, Wisconsin, New York, the New England States, Pennsylvania, and New Jersey on which this bulletin is based was done-during the summers of 1914, 1915, and 1918 , mainly by E. K. Soper, who was assisted in Minnesota by Percy G. Cowin. Data concerning the occurrence of peat in Virginia and North Carolina and the commercial and economic conditions of the peat industry were gathered by C. C. Osbon in 1917, 1918, and 1919. The report was jointly written by Messrs. Soper and Osbon. Some of the information presented is based on the work of the late Charles A. Davis and of the geological surveys and other organizations of the States that contain peat deposits.

The work was done under the general supervision of David White, chief geologist of the United States Geological Survey, to whom the authors are especially indebted for many helpful suggestions. Acknowledgments are due to R. G. Butler for collecting samples of peat and related data in Illinois, Indiana, Ohio, and Michigan in the summer of 1918, to Prof. W. H. Emmons for the loan of field equipment and for other assistance, to Dr. George E. Nichols for valuable data concerning the occurrence of peat and peat moss in Massachusetts, to Dr. George H. Perkins for data concerning the bogs of Vermont, to E. A. Beals for information relating to certain peat deposits in Connecticut, to R. R. Hice and O. E. Jennings for data regarding peat and sphagnum in Pennsylvania, to Alfred Dachnowski for data respecting certain peat areas in Massachusetts, and to Frederick V. Coville and W. C. Alden for reading the entire report and offering valuable suggestions for its improvement. The aid given in the preparation of this report by the operators and engineers of peat plants throughout the country, by numerous State officials not mentioned above, and by others whose personal assistance and courtesy materially contributed to its value is most heartily acknowledged.

\section{PROSPECTS OF THE PEAT INDUSTRY.}

Peat is used in the United States principally in agriculture. Its value as a source of nitrogen for use in the fertilization of the soil seems to have been overlooked by many who are interested in the development of a domestic peat industry. Analyses of the peats of the United States show a content of nitrogen ranging from 1 to 4 per cent and averaging about 2 per cent, much of which can be ex- 
tracted from the peat as a by-product in producer-gas plants or by simple treatment can be made available for plant food without segregating it from the peat. However, a chemical analysis of raw peat is not a true test of its value as a source of nitrogen for agricultural use, for by proper inoculation with nitrifying organisms a substantial quantity of soluble nitrogen is gradually formed and released after the peat has been placed on the soil. Moreover, prepared peat, when added to some soils, either directly or as an ingredient of commercial fertilizer, improves their physical make-up.

Peat has been used commercially in the United States as a fertilizer since 1908, It has been mixed with potash or phosphate, limed, treated with nitrifying bacteria, and applied directly to the soil as a fertilizer, or it has been dried, screened, and used as a nitrogenous ingredient of commercial fertilizers. This subject is now attracting wide attention, and the use of peat in agriculture probably offers greater possibilities in this country than in any other. Raw and strongly acid peat is also used without nitrification in the cultivation of rhododendrons, orchids, blueberries, and other plants that require acid soil and are able to use nitrogen in organic form.

The use of peat and muck as crop soils is being rapidly extended in this country and abroad.

Peat has been used as fuel for centuries, notably in Russia, Denmark, Sweden, Ireland, Germany, and Holland. It was not used as fuel commercially in America before 1908, although immigxants familiar with its use as fuel in their native lands, who came here and settled in our northern States or migrated westward frequently used it for heating and cooking prior to that time. The early settlers in the New England States and some of the pioneers who settled on the prairies of Minnesota, Wisconsin, and northern Iowä before railroads were built and coal could be readily obtained thus made use of peat as fuel.

Although peat in the form of hand-cut blocks was produced as fuel by the early settlers in the United States, the first attempt to manufacture peat fuel commercially in this country was made in 1902, when a strike of the Pennsylvania miners caused a shortage of coal and directed attention to the peat deposits of the country as a source of fuel. Since then many experimental plants have been built, but few have reached the stage of commercial production. The most common causes of failure have been lack of sufficient capital, choice of improper methods and machinery, and injudicious location of the plants where coal was abundant or easily procured.

Although peat fuel has long been profitably produced and sold in Europe, where labor is cheap and coal is scarce, a peat-fuel industry of national proportions would probably not be successful in the 
United States so long as our vast reserve of coal remains available. However, the coal shortage of 1917 and 1918 showed that peat fuel may be profitably made and sold in regions where coal is scarce and expensive, where good peat is abundant, and where, on account of a cold climate and extensive manufacturing industries, large quantities of fuel are needed. Although the prospects for a commercially successful peat-fuel industry in the United States are now probably limited to regions remote from the coal fields, it seems that ultimately, when our reserve of high-grade coal is consumed, there will be a general demand for other fuel, and unless heat and power are obtained from other sources a large peat-fuel industry will be created in this country.

The other commercial uses of peat are numerous and varied. Many valuable by-products, similar to those obtained by distilling coal, may be produced from peat. In the manufacture of stock feed screened peat is used as an absorbent for the uncrystallized residues of beet and cane sugar refineries and as a corrective of intestinal disorders. Owing to the scarcity of raw materials in Europe, fibrous peat is there employed to some extent in making paper, cardboard, artificial wood, and cloth; and sphagnum or peat moss has been extensively used since 1914 as a substitute for absorbent medicated cotton in surgical dressings. In Europe and in the United States use has been made of especially prepared peat and peat moss for mud baths, packing material, and stable litter. A detailed discussion of some of these subjects is given on pages 59-74.

\section{DEFINITIONS.}

The terms "peat" and "muck" are often used interchangeably to designate either of those materials-a practice that is confusing and that should be discouraged. Peat is the partly carbonized organic residuum produced by an arrest in the decomposition of roots, trunks of trees, twigs, seeds, shrubs, mosses, and other vegetation covered or saturated with water. It contains a large proportion of the carbon of the original vegetable matter, and its vegetal structure is generally visible without the microscope. It is usually acidic, and it contains much less inorganic than organic matter. In fact, some pure peats contain less than 4 per cent of inorganic material. Muck is soil that contains a high percentage of uncarbonized organic matter; but, as the name is commonly applied to drained and oxidized areas of peat under cultivation, it is difficult to draw the line between peat and muck; peat may grade into muck and muck into peat. If the material will ignite and burn freely when dry it is usually considered peat. 


\section{ORIGIN OF PEAT.}

GHOLOGIC CONDITIONS FAVORABLE TO THE FORMATION OF PEAT.

Peat is formed under conditions favorable to the profuse growth of plants and to the escape of the plant débris from complete decomposition by bacterial and chemical action. Hence it is clear that the accumulation of this material is governed very largely by topography-that is, the configuration of the surface of the land-and by climate. If the land surface contains depressions, or flat or gently sloping, poorly drained areas in which water may collect and stand permanently, and if the temperature of the air and the soil is low in summer or the humidity of the air is high enough to prevent rapid evaporation, peat-forming plants will flourish.

Glaciation was the dominant factor in the distribution and origin of most of the peat deposits in the United States as well as of many in Canada and Europe. During the glacial epoch a succession of great ice sheets spread southward from Canada over New England, New York, the northern parts of New Jersey and Pennsylvania, and nearly all the region north of Ohio and Missouri rivers. As each sheet in turn melted away it left on the surface of the land irregular deposits of glacial drift consisting of clay or rock flour, sand, gravel, and boulders that had been carried by the moving ice and its attendant waters. In many places this drift so blocked the stream valleys as to form lakes. The unevenness in the thickness of the drift or its irregular settling produced many hollows, and the scouring action of the moving ice on solid rock produced still others.

The earlier ice sheets disappeared so long ago that streams have now drained most of the lands they covered, notably parts of $\mathrm{Ne}$ braska, Kansas, Iowa, Missouri, and Illinois. But though the last ice sheets, those of the Wisconsin stage, melted ages ago the streams have not yet had time to clear out and extend their valleys so as to drain the thousands of lakes and ponds and the hundreds of thousands of acres of marsh and swamp land in the region covered by the last glaciers. For this reason the peat bogs are confined largely to the area of the latest glacial drift, though basins in which peat may accumulate are also formed in limestone regions by solution.

The factors next in importance to glaciation and the kind of surface rock in forming deposits of peat are wave and stream action and coastal subsidence. Many peat deposits of salt-marsh and freshwater origin are seen in drowned valleys, where the coast has subsided and landlocked lagoons or deltas have been formed, and in flat, imperfectly drained areas farther inland. In some places saltmarsh peat overlies peat of fresh-water origin, indicating coastal subsidence. 
Abundant and well-distributed rainfall, high humidity, and a cool or moderate temperature are the most favorable climatic conditions for the formation of peat. These conditions are found in the region of the Great Lakes, in the New England States, and, in varying degrees of competency to form peat, in the Atlantic Coast States. If the climate is otherwise suitable, peat forms at some places in the Frigid Zones, but not extensively. In the Torrid Zone, although, owing to the high temperature, plant débris decays rapidly, despite the profuse plant growth peat accumulates slowly and therefore contaîns a high percentage of inorganic matter.

On account of the rapid run-off of surface waters in the Appalachian and Rocky Mountain regions little valuable peat is formed, and scanty rainfall produces the same result in the area between the Rocky Mountains and the eastern part of the Dakotas. Contrary to the general belief, there are few commercially valuable deposits of peat in the lower Mississippi Valley, perhaps because of the high temperature and the vast amount of alluvium carried by the Mississippi and its tributaries and deposited from time to time on its flood plain.

\section{CHARACTER OF PEAT-FORMING VEGETATION.}

As peat consists of partly decayed and disintegrated plant débris, the function performed by plants in the origin of peat is fundamental. Although hundreds of different plants have been identified in the bogs and swamps of this country and thousands of species have contributed material to form peat, comparatively few plants contribute the greater part of the vegetable débris that makes it. The most common of these plants are trees, heath shrubs, sedges and grasses, mosses, pondweeds, water lilies, reeds, cat-tails, algae, and ferns. In a favorable environment most of these plants multiply rapidly and soon predominate over their competitors; in fact, in some areas they grow so densely that man can penetrate them with diffculty, and an immense quantity of dead vegetation annually accumulates.

\section{CHEMICAL CHANGES IN PEAT-FORMING MATTER.}

One of the chief substances formed by plants during their growth is cellulose $\left(\mathrm{C}_{72} \mathrm{H}_{120} \mathrm{O}_{80}\right)$, which consists of carbon, hydrogen, and oxygen. These constituents are absorbed by the leaves from the atmosphere and by the roots from the soil. Cellulose, because of its complex composition, is an unstable compound and when attacked by fungi and bacteria decomposes rapidly. If at the end of the growing season the plant débris falls upon drained soil it is vigorously attacked by these microorganisms, and the carbon and hydrogen of the cellulose unite with the atmospheric oxygen and with 
each other, forming carbon dioxide, water, and marsh gas. In other words, if oxidation is unhampered, the organic matter will disappear in a relatively short time. If, however, the plant matter falls into water or upon soil saturated with moisture, it undergoes a change different from the decay suffered by exposed vegetation. The atmospheric oxygen is largely excluded, and as the activity of fungi and bacteria is controlled by the supply of air, upon which they depend for their existence, decay is slow, the plant débris becomes buried, and a large proportion of the fixed carbon is retained. The salient features in the production of peat $\left(\mathrm{C}_{62} \mathrm{H}_{72} \mathrm{O}_{24}\right)$ from cellulose $\left(\mathrm{C}_{72} \mathrm{H}_{120} \mathrm{O}_{80}\right)$ are the elimination of hydrogen and oxygen as water $\left(\mathrm{H}_{2} \mathrm{O}\right)$ and of carbon and oxygen as carbon dioxide $\left(\mathrm{CO}_{2}\right)$ and the generation of methane $\left(\mathrm{CH}_{4}\right)$. This is the process of carbonization.

If the surface conditions are unchanged, carbonization is largely arrested with the formation of peat, and the accumulation of organic matter may exist indefinitely as peat, unless the land is drained and decomposition begins again or unless the peat is deeply buried beneath superposed deposits, generally muds, sands, limestone, and other sedimentary beds, and subjected to pressure, accompanied by heat. Lignite, bituminous coal, anthracite, and graphite are succeeding stages in the process of carbonization of the buried vegetable débris. Most coals were once peats; most coal fields were formerly swamps, and the formation of peat in the bogs and swamps of this country to-day is an example of the first stage in the process of coal formation. Deposits essentially similar were laid down in many parts of the United States during the Carboniferous. Triassic, Cretaceous,'and Tertiary periods.

\section{CLASSIFICATION OF WET LANDS AND PEAT DEPOSITS.}

\section{TYPES OF UNDRAINED IAND.}

The terms "bog," "marsh," and "swamp" are often used interchangeably to indicate the same kind of undrained land surface. It is suggested that, for the sake of exactness, these terms should be used to designate the following types of undrained lands:

Bog.-A flat or gently sloping wet area devoid of trees, except in some places small scattered patches of tamarack or black spruce, and overgrown principally by sphagnum moss and heath shrubs or by grasses and sedges. The numerous sphagnum areas of Minnesota, Wisconsin, and the New England States belong to this class.

Marsh.-An open shallow basin or relatively flat area covered with water, devoid of trees, and overgrown by grasses, sedges, cattails, bulrushes, or reeds. The chief difference between bogs and 
marshes lies in the character of the living vegetation and the quantity of surface water. Marshes often adjoin lakes, rivers, and seacoasts and may contain either fresh or salt water. The Revere Marsh, near Boston, Mass., is a typical salt marsh, and the marshes adjoining Charles and Neponset rivers in Massachusetts are good examples of the fresh-water type.

Swamp.-A low, flat area covered or saturated with water and overgrown by trees, with or without an undergrowth of shrubs. The surface may be overgrown by a thick mat of vegetation, consisting of small plants, a condition found in many of the swamps of New England, or, as in some parts of Dismal Swamp, Va., it may be covered with water and comparatively free from small plant growth. Swamps are sometimes named from the trees that predominate in them, as spruce swamp, cedar swamp, gum swamp, and cypress swamp. In swamps containing standing water the contribution of sphagnum moss and heath shrubs to the débris from which peat. forms is small.

\section{KINDS OF PEAT DEPOSITS.}

TOPOGRAPHIC TYPES.

As classified according to topography, there are three general types of peat deposits - the filled basin, in which the peat accumulates in marshes, ponds, and lakes; the built-up deposit and its corollary, the climbing bog, in which the peat forms on flat or gently sloping moist areas not covered with waterimand the composite area consisting of built-up peat underlain by peat of the filled-basin type. Plate II illustrates the first two types. Although deposits of all three kinds are found throughout the peat regions, the filled-basin type predominates. Built-up peat forms on areas where the drainage is so greatly interrupted that the soil becomes permanently saturated with moisture. As shown by deep test borings, large areas now covered with built-up peat are underlain by fine-grained peat composed of the remains of aquatic plants, indicating that the built-up stage was preceded by a long period of subaqueous peat formation. The most extensive deposits of lake and built-up peat are in the Great Lakes region and the New England States; and the most extensive deposits of marsh peat are in the Atlantic Coast States. Large climbing bogs are found in Maine, and some are found in other New England States.

FLORAL TYPES.

As classified according to dominant plant growth, the eight common types of peat areas are as follows: (1) Pondweed basins, (2) 


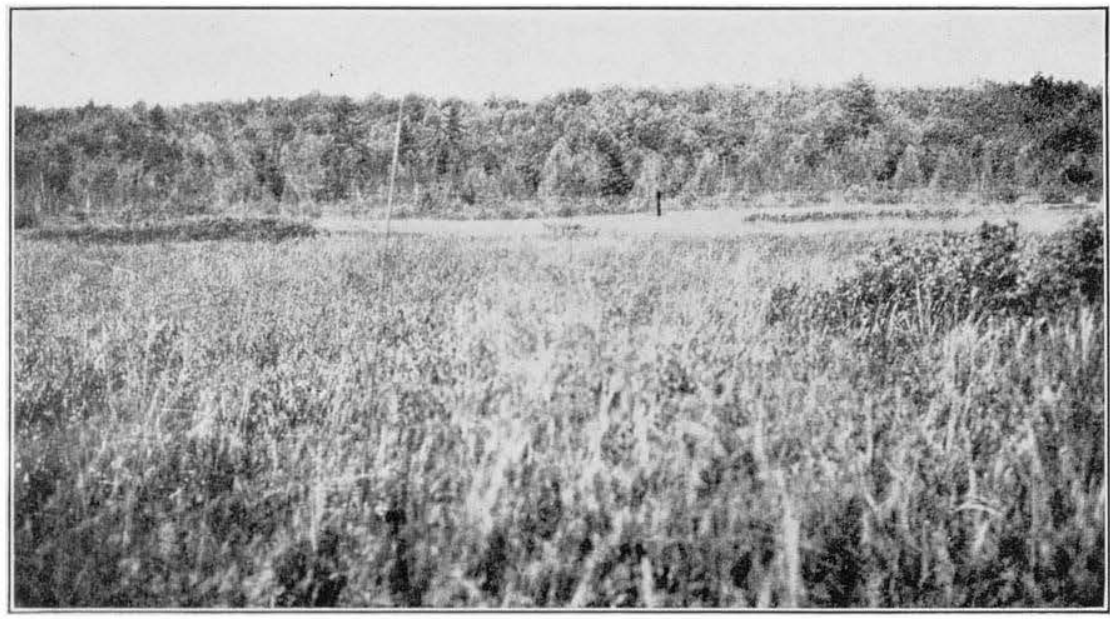

A. FILLED-BASIN PEAT DEPOSIT.

The old shore line ( 1 ) is visible.

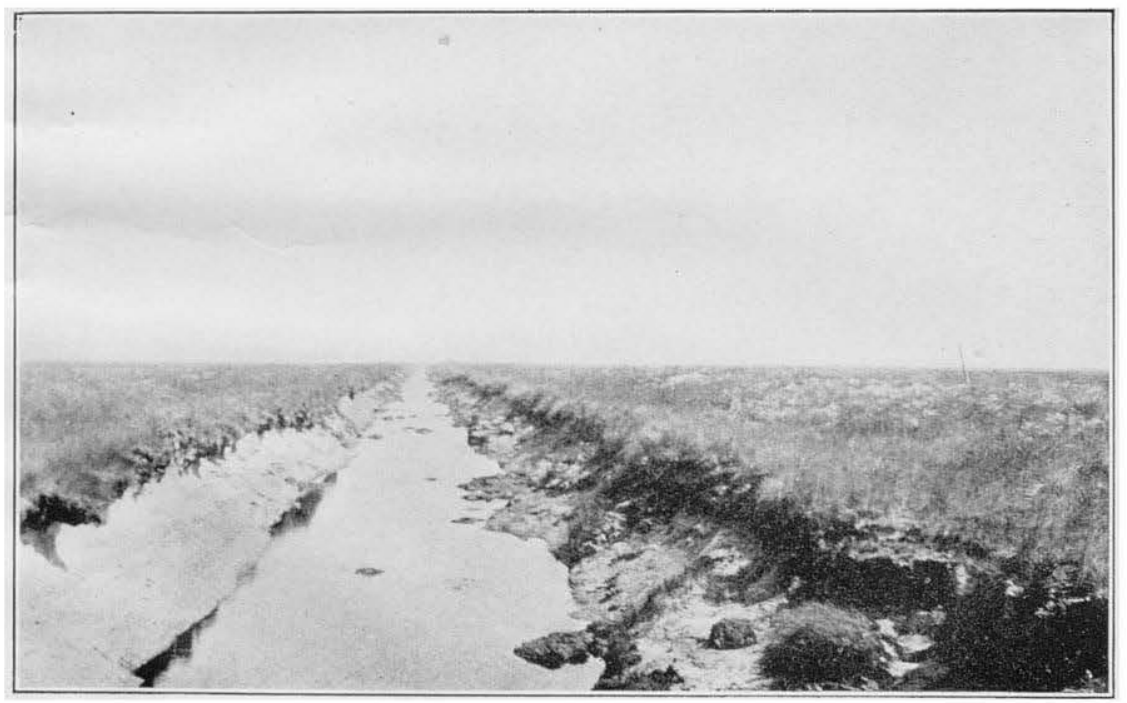

F. BUILT-UP PEAT DEPOSIT FORMED ON A RELATIVELY FLAT SURFACE.

Types of Mature Peat Deposits 

grass-sedge marshes, (3) sphagnum-heath bogs, (4) cedar swamps, (5) spruce swamps, (6) tamarack swamps, (7) gum swamps, and (8) cypress swamps.

\section{PEAT-FORMING FLORAS.}

FILLED BASINS.

Beginning at the center of basins filled with water and proceeding shoreward, the usual sequence of vegetation, depending to a large extent upon the depth of the water, is algae and stoneworts, pondweeds, water lilies, bulrushes, and amphibious sedges. (See Pl. III.) Sphagnum and heath shrubs sometimes grow on the marginal zones and are abundant on the quaking bogs of the Northern States. In the northern peat region a growth of tamarack and spruce is often found near the water, and in the Atlantic coastal area a zone of gum and cypress, but these trees are not large contributors to the filledbasin peat. Adjoining zones of vegetation usually overlap, and some plant zones may be absent. As the quantity of surface and underground water and the acidity or alkalinity of the underground water largely determine the flora of a region, and as the gradual accumulation of peat lessens the depth of the water in a peat-forming basin, the vegetal sequence mentioned gives a fairly reliable index to the general dominant plant associations that successively enter into the development of a filled-basin peat deposit.

The following stages of plant growth usually predominate in the order given in peat-forming basins that contain alkaline waters: (10) Ctopewort and water weed (Chara-Philotria association), (2) pondweed and water lily (Potamogeton-Nymphaea association), (3) rush and wild rice (Scirpus-Zizania association), (4) meadow sedge and grass (Carex association).

Some filled-basin peat deposits, however, because of the influence of fire, drought, and drainage, were not formed by the successive growth and decay of all these plant associations. Changes in the surface water from alkaline to acid also affect the composition of the floras. Fine-grained algal peat is usually found at the bottom of filled-basin deposits, indicating that they had at least a normal origin. This material is generally overlain by strata of weed, sedge, and grass peat. Algal peat is usually not found in shallow filledbasin deposits, showing that the second plant association took root in the shallow water soon after the basin was formed and prevented the dominance of algae and other cryptogamic plants. The mass of peat in some filled-basin deposits consists of fibrous material produced chiefly by the decomposition of sedges and grasses, indicating that the meadow stage predominated for a long time. If a filled- 
basin peat deposit is destroyed by fire, the first stage in the usual succession of plant growth is often resumed after the basin is again filled with water, but if subjected to drainage or drought the earlier stage in the succession of vegetation may, if like conditions prevail, be replaced by bog-heath plants.

\section{BUILT-UP BOGS.}

As built-up peat accumulates on level or gently sloping surfaces the plant associations that successively predominate in peat-forming basins do not materially enter into its formation. As shown by Plate III, the stages of plant growth that contribute to built-up peat are as follows: (1) Bog-heath (Andromeda-Ledum association); (2) tamarack-spruce (Larix-Ledum association) in the northern region; or (3) gum-cypress (Ny88a-Taxodium association) in parts of the Atlantic coastal region.

When built-up deposits are consumed by fire the process of peat formation again begins with the bog-heath stage, unless the area surrounding it is overgrown with trees, and then it is sometimes resumed with the more advanced stage. The permanent flooding of a built-up bog would convert it into a marsh, and if drained to the depth of a few feet the formation of peat in built-up bogs is usually stopped.

As built-up deposits are largely formed by heath shrubs and mosses, the peat usually consists of the remains of only a few plant forms and is therefore relatively homogeneous. Often the peat con: sists almost entirely of partly decayed sphagnum, especially in the coniferous forest region. Some built-up deposits are overgrown by plants of the same kind that formed the mass of the peat in them, indicating uniform climatic and topographic conditions for a long time. In many deposits, however, the floral development is more advanced and heath shrubs and trees predominate. Many extensive built-up bogs in the coniferous forest areas of the Northern States are in the heath or tree stage.

\section{COMPOSITE AREAS.}

As composite peat areas consist of filled-basin peat overlain by peat of built-up origin they can not be distinguished from built-up deposits by surface criteria. Their history can be ascertained only by numerous test borings and by careful study of the topography and of the plant remains. All the normal stages of plant development may enter into the formation of composite peat, and many of the deposits therefore contain successive strata of algal, rush, grass, sedges, sphagnum, shrub, and tree peat. 


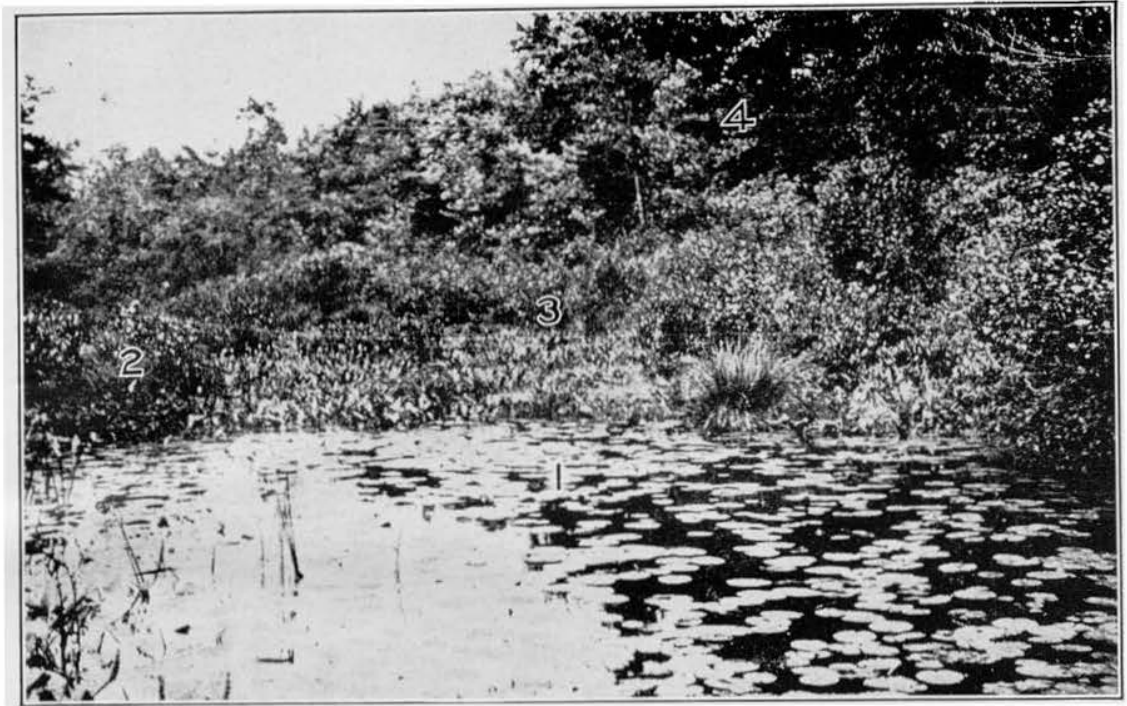

A. SUCCESSIVE ZONES OF GROWTH OF (1) PONDWEED AND WATER LILY; (2) SEDGE; (3) SHRUB; AND (4) FOREST TREES.

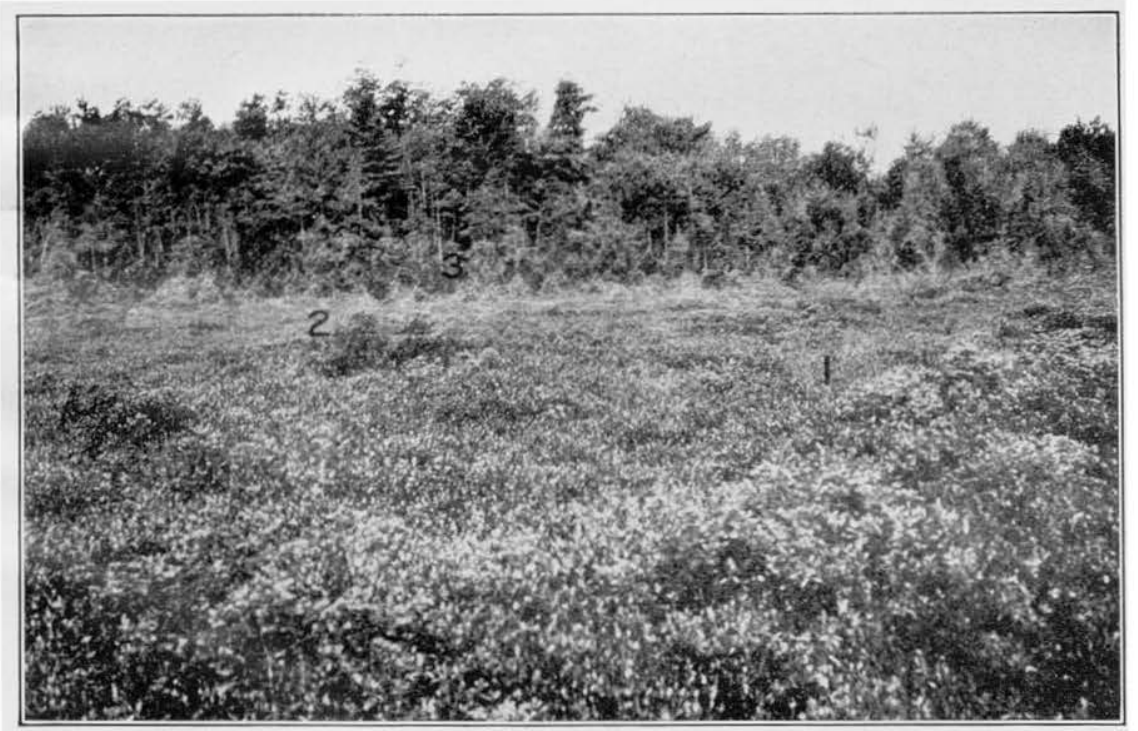

B. SUCCESSIVE ZONES OF GROWTH OF (1) HEATH GRASSES; (2) SHRUBS; AND (3) FOREST TREFS.

Peat-Forming Basins. 



\section{PROCESSES OF FORMATION.}

\section{FILIED BASINS.}

Fresh-water peat.-Most of the peat deposits of this country were formed by the accumulation of plant débris in ponds, fresh-water marshes, and lakes and are called filled-basin deposits. (See Pl. III.) The numerous peat-forming lakes and ponds in all developmental stages found in many parts of the Great Lakes and New England States afford ample evidence of the processes by which the other deposits formed. Test borings usually show that fine-grained peat produced by the decomposition of algae and other cryptogamic plants, the first to grow in deep water, constitutes the bottom layer of the deep deposits. Coincident with the formation of algal peat in the deep water, pondweeds, water lilies, sedges, and other plants that take root in shallow water established themselves on the margins of the basins. Thus the basins were gradualiy filled with plant débris from the bottom and from the shores. The formation of algal peat is relatively slow, but after the water becomes shallow enough for the growth of pondweeds and sedges in all parts of the basin the peat accumulates rapidly and soon fills the depression. When and where the surface of the deposit is raised to the general level of the surrounding country, herbs, sphagnum, and heath shrubs appear, and if moisture is abundant, the filled-basin process will be followed by the formation of built-up peat.

Salt-marsh peat.-Salt-marsh peat, though formed in practically the same manner as fresh-water peat, differs from it somewhat in character. Fow seed plants tolerate salt water, and the number of plant varieties found in salt marshes is therefore rather small. The most common types are salt-marsh grasses, rushes, and sedges. The entire vegetation of some of the New England salt marshes consists of one dominant and two or three subordinate species.

In some of the coastal marshes of New England salt-marsh peat is underlain by peat of fresh-water origin, indicating the subsidence of that part of the Atlantic coast. Bastin and Davis ${ }^{1}$ in discussing the origin of certain peat deposits on the coast of Maine said in substance:

Some persons think that these overlying strata of salt-water peat were formed in bays or inlets which had been cut off from the ocean by barrier beaches and to which salt water was subsequently admitted by wave or current action. This explanation, however, is not satisfactory, because the salt-water peat, which was formed from the remains of plants similar to those now growing in these marshes, plants that can not exist even a few inches above their present level, is 3 feet thick or more in many places. It therefore seems improbable

\footnotetext{
1 Bastin, E. S., and Davie, C. A., The peat deposits of Maine: U. s. Geol. Survey Bull.
} 876, pp. 20-21, 1809. 
that the salt marshes in which this material accumulated were made by the action of waves or currents. A more plausible explanation of the character of these deposits presupposes the gradual subsidence of a coast containing scattered fresh-water marshes in which peat was forming. As the sinking of the coast continued these marshes were filled with salt water, and peat formed from the decay of salt-water plants was deposited upon the fresh-water peat. If this theory is correct the thickness of the salt-water peat indicates that the coast has been sinking at the rate of about a foot a century. As the freshwater deposits are both underlain and overlain by salt-water peat in some places it seems that a slight uplift of the coast preceded the present period of subsidence.

\section{BUILT-UP BOGS.}

Peat deposits formed by the accumulation of plant matter on level or gently sloping surfaces are called built-up bogs. Plate II illustrates a typical built-up bog. Mosses, grasses, herbs, and heath shrubs contribute the dead vegetation, and the water, though it may never rise above the surface, is progressively elevated as the peat collects. Surface conditions are little changed from year to year, and hence built-up bogs are relatively homogeneous in structure.

\section{COMPOSITE AREAS.}

When a basin deposit is filled with peat to the level of the surrounding country it is mature, and if the moisture is sufficient the built-up process begins. Mosses, herbs, and heath shrubs displace the pondweeds, water lilies, sedges, and like plants, and thenceforth the deposit develops the same as if it had originated on a relatively level land surface not covered with peat. The result is a composite peat deposit. The peat may accumulate to a thickness of many feet above the former water level in the hasin. Composite areas are recognized by a marked change in the structure of the peat where the pondweeds, water lilies, and sedges were displaced by bog plants.

\section{RATE OF FORMATION.}

As the formation of peat depends upon many factors the rate of its accumulation varies widely from year to year. If the climate, topography, and vegetation are favorable, peat forms rapidly; but if one or more of these is relatively unfavorable, the rate of accumulation is retarded. Although most of the large deposits of peat in the United States have been examined, no definite evidence has yet been obtained to show the rate of their formation. Even under the most favorable conditions it is too slow to be measured by ordinary observation. Persons who have lived near peat-forming basins for half a century are unable to see the slightest change in the appearance or depth of the peat, although careful examination shows that it has been forming continuously. Dana ${ }^{2}$ gives the rate as 1 foot in 5 or 10

2 Dana, J. D., Manual of geology, 4th ed., p. 154, 1895. 
years, but this probably far exceeds the average rate of formation in the United States. The only reliable means of making even a rough estimate of the rate at which peat is formed seems to be to estimate the average thickness of some of the largest deposits in the Great Lakes States and the time that has elapsed since the Wisconsin stage of glaciation. As already stated, most of the peat deposits of the Great Lakes States originated in glacial lakes and ponds or on flat, poorly drained areas formed by topographic changes due to glaciation. By assuming that peat began to accumulate in certain typical lakes and ponds soon after the final recession of the ice sheet, that 10,000 to 30,000 years have elapsed since the close of Wisconsin glaciation, that the average thickness of these deposits is about 18 feet, and that the formation of the peat was uninterrupted, we may compute the average rate of accumulation per century at 0.72 to 2.16 inches. But as field study shows that fire, flood, and drought have interrupted the formation of peat in many of the large deposits these figures are only speculative, and as the water level in practically all peat areas varies with fluctuations in annual precipitation (and such variations materially affect the formation of peat) it is evident that the rate of formation in most peat deposits has not been uniform.

\section{PROPERTIES OF PEAT.}

\section{PHYSICAL COMPOSITION.}

GENERAL FEATURES.

Native peat consists of partly decayed vegetable matter, inorganic minerals, and water in varying proportions, the usual ratio being 10 per cent of solid matter to 90 per cent of water. In specific gravity it ranges from 0.1 to 1.06 and in weight from 7 to 65 pounds per cubic foot. Aside from its high water content, peat is extremely variable, and scarcely any two deposits contain material that is exactly similar in physical properties. This diversity is due to many causes, the most notable of which are the variety of plants from which the peat was formed, and differences in climate, in the ages of the deposits, in water level, and in the quantity of sediment deposited during the accumulation of the peat.

\section{TEXTURE.}

The texture of peat depends upon the kinds of plants from which it was formed and the physical conditions under which it accumulated. Peat formed from algae and mosses is fine grained and comparatively homogeneous, whereas peat produced by the decay of grasslike or woody plants is generally fibrous and poorly decomposed unless decay has progressed unusually far. Peat formed by the decomposition of shrubs and trees is generally woody in struc- 
ture. Dead vegetation of any kind that is exposed for long periods to the free action of fungi and bacteria becomes thoroughly disintegrated and fine in texture. Peat that accumulates in river valleys and lakes whose water contains much sediment is usually too impure and contains too much ash for commercial use.

The following classification ${ }^{3}$ of peats by physical characteristics includes all types found in the United States:

Turfy peat.-Consisting of slightly decomposed mosses and other peat-producing plants, having a yellow or yellowish-brown color, very soft, spongy, and elastic; specific gravity, 0.11 to 0.26 , the full English cubic foot weighing from 7 to 16 pounds.

Fibrous peat.-Unripe peat which is brown or black in color, less elastic than turfy peat, the fibers either of moss, grass, roots, leaves, or wood, distinguishable by the eye, but brittle and easily broken; specific gravity, 0.24 to 0.67 , the full cubic foot weighing, accordingly, from 15 to 42 pounds.

Earthy peat.-Nearly or altogether destitute of fibrous structure, drying to earthlike masses which break with more or less difficulty, giving lusterless surfaces of fracture; specific gravity, 0.41 to 0.90 , the full cubic foot weighing from 25 to 56 pounds.

Pitchy peat.-Dense; when dry, hard; often resisting the blows of a hammer, breaking with a smooth, sometimes lustrous fracture into sharp-angled pleces; specific gravity, 0.62 to 1.03 , the full cubic foot weighing from 38 to 65 pounds.

The peat deposits of Minnesota, Wisconsin, and Michigan were produced chiefly by the decomposition of mosses, sedges, grasses, heath shrubs, and trees, and their texture varies from fibrous in the upper layers to plastic in the lower. The deposits of Iowa, Illinois, Indiana, Ohio, Pennsylvania, New York, and New Jersey consist* largely of the remains of grasses, mosses, rushes, cat-tails, and reeds, and are somewhat similar in texture. On the whole, however, the peats of the second group, those of the southern Lake States, are more fibrous than those of the first, and, except in northern Indiana and northeastern Pennsylvania, are relatively free from the remains of sphagnum moss. Sphagnum peat is abundant in the bogs of northern Minnesota, Wisconsin, and Michigan and in Maine, and some is found in the other New England States and New York. Pondweeds, pond lilies, heath shrubs, rushes, cat-tails, and coniferous trees were also large contributors to the peats of Maine and of the other New England States. Grasses are the dominant form of plant life in the salt marshes on the New England coast, notably the genus Spartina, and grass peat is therefore abundant in this area. The peats of the Atlantic Coastal States south of New Jersey are very different in composition and texture from those of the Great Lakes region and the New England States. There is relatively little sphagnum in the Atlantic coastal region, and both coniferous and deciduous trees con-

3 Johnson, 8. W., Peat and Its uses, pp. 95-96, New York, 1866. 
tributed a large proportion of the vegetable débris from which the peat was formed.

COLOR.

Peat ranges in color from light yellow through various shades of brown to jet black, the color representing in a measure the degree of decomposition. Peat that is new or that has been well protected from the air is usually light yellow or brown; well-decomposed humified peat is jet black. Green peat, produced by the decomposition of algae and related aquatic plants, is found at the bottom of some filled-basin deposits. On drying in the air most peats become brighter in color, except the very light varieties, which usually change to dark brown or black after being macerated and dried. Peat that is red, gray, or white in spots or feels very gritty when crushed between the teeth contains too much inorganic matter for commercial use as a fuel.

\section{WATER-HOLDING CAPACITY.}

The affinity of peat for moisture is proverbial. In fact, as previously explained, peat can not form unless the plant débris is saturated or covered with water. The peat in most deposits contains about 90 per cent of moisture, which is held both mechanically and chemically in the plant cells and intercellular spaces. In other words, a short ton of typical raw peat consists of about 200 pounds of solid matter to 1,800 pounds of water. The reduction of this high content of moisture is the paramount necessity in the commercial utilization of peat. Many attempts have been made to remove the excess moisture by compression, but it resists the strongest pressure obtainable and can be materially reduced economically only by evaporation.

Relation between solid and liquid constituents of a ton of typical raw peat as its water content is progressively reduced from 90 to 10 per cent. ${ }^{a}$

\begin{tabular}{|c|c|c|c|c|c|}
\hline $\begin{array}{l}\text { Per cent } \\
\text { of water } \\
\text { in peat. }\end{array}$ & $\begin{array}{l}\text { Quantity } \\
\text { of water } \\
\text { eliminated } \\
\text { for each } \\
\text { reduction of } \\
10 \text { per cent } \\
\text { (pounds). }\end{array}$ & $\begin{array}{l}\text { Cumulative } \\
\text { quantities } \\
\text { of water } \\
\text { eliminated } \\
\text { (pounds). }\end{array}$ & $\begin{array}{l}\text { Weight of } \\
\text { peat aft er } \\
\text { each } 10 \\
\text { per cent } \\
\text { reduction } \\
\text { of water } \\
\text { content } \\
\text { (pounds). }\end{array}$ & $\begin{array}{l}\text { Quantity } \\
\text { of water } \\
\text { (pounds). }\end{array}$ & $\begin{array}{l}\text { Quantity } \\
\text { of solid } \\
\text { matter } \\
\text { (pounds). }\end{array}$ \\
\hline $\begin{array}{l}90 \\
80 \\
70 \\
60 \\
50 \\
40 \\
30 \\
20 \\
10\end{array}$ & $\begin{array}{r}1,000.0 \\
333.3 \\
166.7 \\
100.0 \\
66.7 \\
47.6 \\
35.7 \\
27.8\end{array}$ & $\begin{array}{l}1,000.0 \\
1,333.3 \\
1,500.0 \\
1,600.0 \\
1,666.7 \\
1,714.3 \\
1,750.0 \\
1,777.8\end{array}$ & $\begin{array}{r}2,000.0 \\
1,000.0 \\
666.7 \\
500.0 \\
400.0 \\
333.3 \\
285.7 \\
250.0 \\
222.2\end{array}$ & $\begin{array}{r}1,800.0 \\
800.0 \\
466.7 \\
300.0 \\
200.0 \\
133.3 \\
85.7 \\
50.0 \\
22.2\end{array}$ & $\begin{array}{l}200 \\
200 \\
200 \\
200 \\
200 \\
200 \\
200 \\
200 \\
200\end{array}$ \\
\hline
\end{tabular}

a Davis, C. A., Uses of peat for fuel and other purposes: Bur. Mines Bull. 16, p. $110,1911$. 
The accompanying diagram (fig. 1), which is based on the foregoing table, shows graphically the relation between the weight and water content of raw peat.

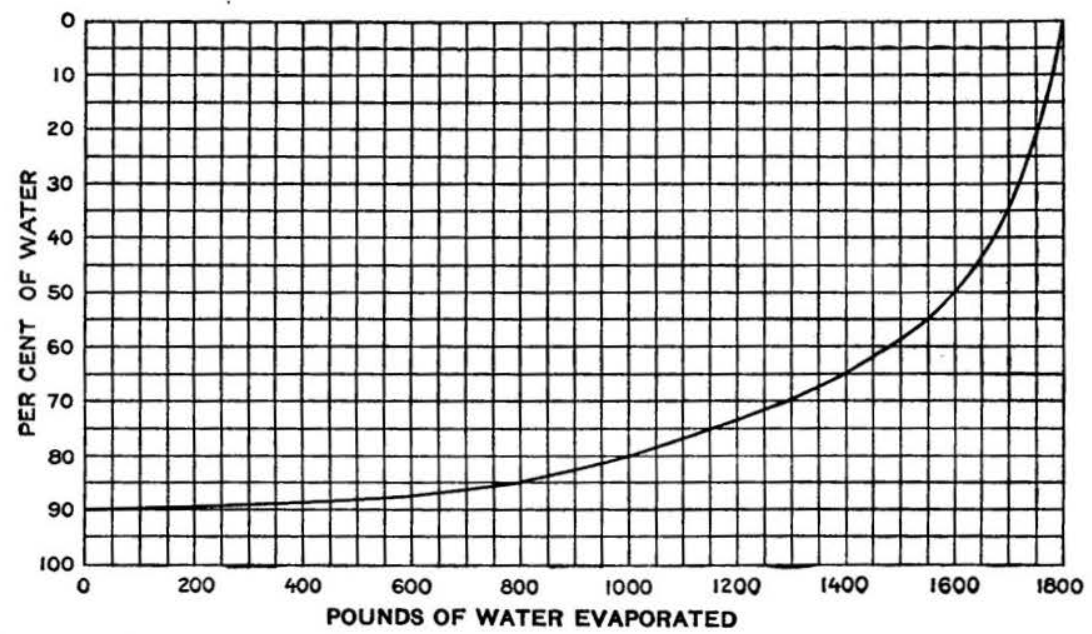

FIGURm 1.-Diagram showing relation between the weight and water content of a ton of raw peat at different stages of evaporation.

\section{CHEMTCAL, COMPOSITION.}

\section{GENERAL FEATURES.}

A detailed study of the chemical properties of more than 500 samples of peat taken from deposits in different parts of the peat regions of this country leads to the following conclusions:

Peat consists of carbon, hydrogen, oxygen, and relatively. smuall quantities of nitrogen. Although the exact atomic relations of its principal elements are not known and probably are not constant, the formula $\mathrm{C}_{62}^{\prime} \mathrm{H}_{72} \mathrm{O}_{24}$ is typical. The composition of peat is illustrated by the following analysis (ash and moisture omitted) :

Composition of peat.

Carbon

59.50

Hydrogen

5.50

Oxygen

$33.00^{\circ}$

Nitrogen

2. 00

100.00

The quantity of "fixed" or "free" carbon generally ranges from 10 to 60 per cent, the remainder being combined with other elements. Volatile matter usually ranges from 25 to 70 per cent and moisture from 15 to 30 per cent in air-dried peat. As the volume of oxygen is relatively high, good peat ignites readily and burns freely, leaving 
little unconsumed residue. Sulphur usually ranges from 0.2 to 0.6 per cent and nitrogen from 1 to 4 per cent, the average for nitrogen being about 2 per cent.

The ash in native peat, which renders it more or less impure, constitutes from 3 to 30 per cent of its dry weight and is traceable either to the plant cells or to the mineral matter carried in suspension or solution by the water in which the peat formed. The inorganic impurities of peat consist of silica, alumina, iron oxide, magnesia, lime, soda, potash, sulphuric acid, chlorine, and phosphoric acid. If the ash content exceeds 8 per cent, it is due to the mineral matter in the water that covered the peat during formation, and it usually consists of silica in the form of sand or silt or of alumina and silica in the form of clay. Mineral constituents other than silica and alumina in excess of 8 per cent are not common in peat and where found may be traced to the local ground and surface waters. The ash content of the best peats in the United States ranges from about 6 to 12 per cent, though many of the largest deposits in the Great Lakes area contain 15 per cent.

The following table shows the composition of the ash in three samples of typical New England peat ${ }^{4}$ :

Analyses of the ash in Connecticut peat.

\begin{tabular}{|c|c|c|c|}
\hline Inorganic impurities. & 1 & 2 & 3 \\
\hline \multirow[t]{2}{*}{ 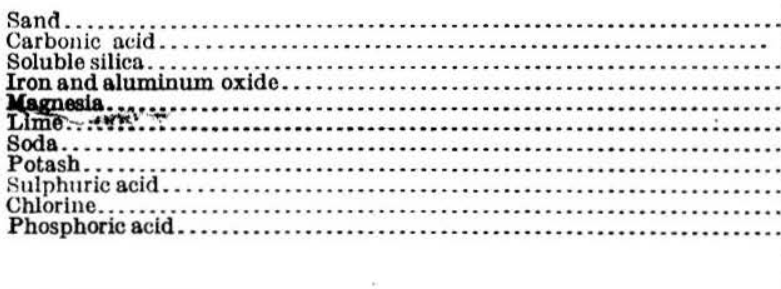 } & $\begin{array}{r}12.11 \\
19.60 \\
8.23 \\
5.17 \\
6.06 \\
41.39 \\
.58 \\
.69 \\
5.52 \\
.15 \\
.50\end{array}$ & \begin{tabular}{r|}
15.04 \\
22.28 \\
1.40 \\
9.08 \\
4.20 \\
35.59 \\
.00 \\
.80 \\
10.41 \\
.43 \\
.77
\end{tabular} & $\begin{array}{r}67.01 \\
15.59 \\
1.05 \\
6.60 \\
\text { Tr8ce. } \\
3.46 \\
4.04 \\
.70 \\
1.55\end{array}$ \\
\hline & 100.00 & 100.00 & 100.00 \\
\hline
\end{tabular}

If the inorganic impurities of decayed vegetation are much in excess of 30 per cent the material should be classed as muck rather than peat.

\section{VALUE OF PEAT AS A FERTIUIZER.}

The value of peat in soil fertilization lies in its content of nitrogen and humus and in the beneficial mechanical effect it produces on certain lands. Black, well-decomposed peats are the most satisfactory ordinary fertilizers, for they are generally heavier and more compact and contain more nitrogen and less fibrous material than the brown peats. Raw, strongly acid peats, however, should be used in fertilizing soil intended for the growth of acid-tolerant crops.

‘Johnson, S. W., Peat and its uses, pp. 47-49, New York, 1866. 
VALUE OF PEAT AS A FUHL.

The value of peat as fuel is dependent upon many factors, chief of which are the degree of decomposition, heating value, and ash content. Coarse-textured, fibrous peat is inferior for fuel to black, compact, thoroughly decomposed peat, except kinds that contain a very large proportion of ash. The heating value of good moisturefree peat, which ranges from 7,000 to 10,000 British thermal units per pound, is determined chiefly by its content of fixed carbon and ash. Though the ash is inert, it displaces an equal volume of combustible matter and absorbs heat in maintaining its temperature at the same degree as the accompanying carbon. Salt-marsh peat generally contains a large quantity of sodium chloride and other inorganic minerals, and is therefore of little value as fuel. The maximum quantity of ash that is usually considered allowable in peat for commercial use as fuel has been placed between 20 and 25 per cent, but if it exceeds 20 per cent of the total dry weight the peat is scarcely worth the labor of production. Though peat containing from 10 to 12 per cent of ash is good, in Ireland peat is not considered first-class fuel if the ash content exceeds 5 per cent.

The following table shows the calorific value of peat as used commercially compared with other fuels:

Comparative calorific value of peat and other fuels.

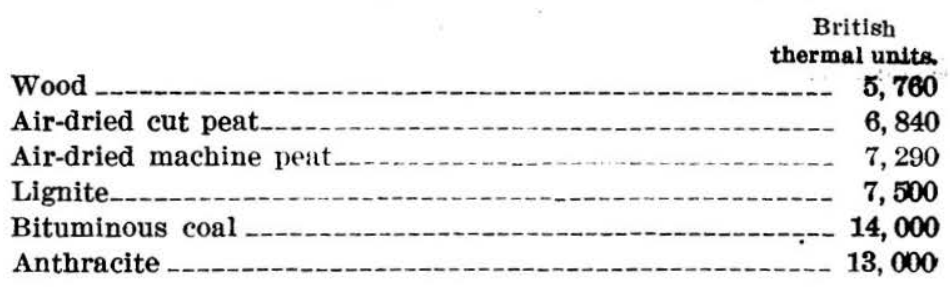

In calorific value a ton of machine peat is equal to about 1.3 tons of wood, 0.5 ton of bituminous coal, and 0.6 ton of anthracite.

ANALYTICAL METHODS.

The chemical composition of peat, like that of coal, is usually determined by proximate and ultimate analyses. Percentages of moisture, volatile matter, fixed carbon, and ash are shown by the proximate analysis, and those of hydrogen, carbon, nitrogen, oxygen, sulphur, and ash by the standard ultimate analysis. The calorific value of the peat, both as received in the laboratory and moisture free, is then determined in calories, from which the value in British thermal units is calculated. The heating value of peat of any moisture content up to 30 per cent may be ascertained by deducting for each per cent of moisture 1 per cent from the calorific value determined as of water-free peat. A full description of the methods of making 
chemical and calorimetric analyses of peat is given in Technical Paper 8 and Bulletin 16 of the United States Bureau of Mines.

The analyses of samples taken during the progress of field work for this report were made under the supervision of $\mathrm{H}$. M. Cooper, of the United States Bureau of Mines. The samples were air-dried to about 50 per cent moisture in the field and sent to the laboratory. in canvas bags.

\section{ANALYSES.}

The following tables give a large number of analyses of moisturefree peat made for this report, as well as of raw samples shipped to the laboratory in air-tight containers and tested in their native condition. Many of the tables were taken from Bureau of Mines Bulletin 16 and from reports issued by State geological surveys and other organizations.

\section{CONNECTICUT.}

Analyses of moisture-free peat and muck.

[H. M. Cooper, Buresu of Mines, snalyst.]

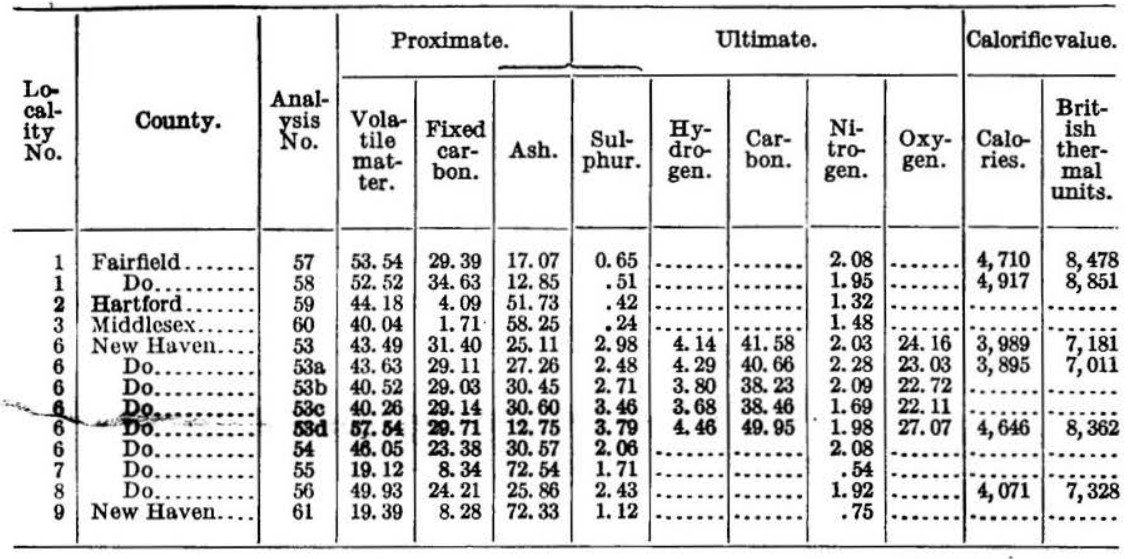

Analyses of moisture-free peat.

[Furnished by E. A. Beals.]

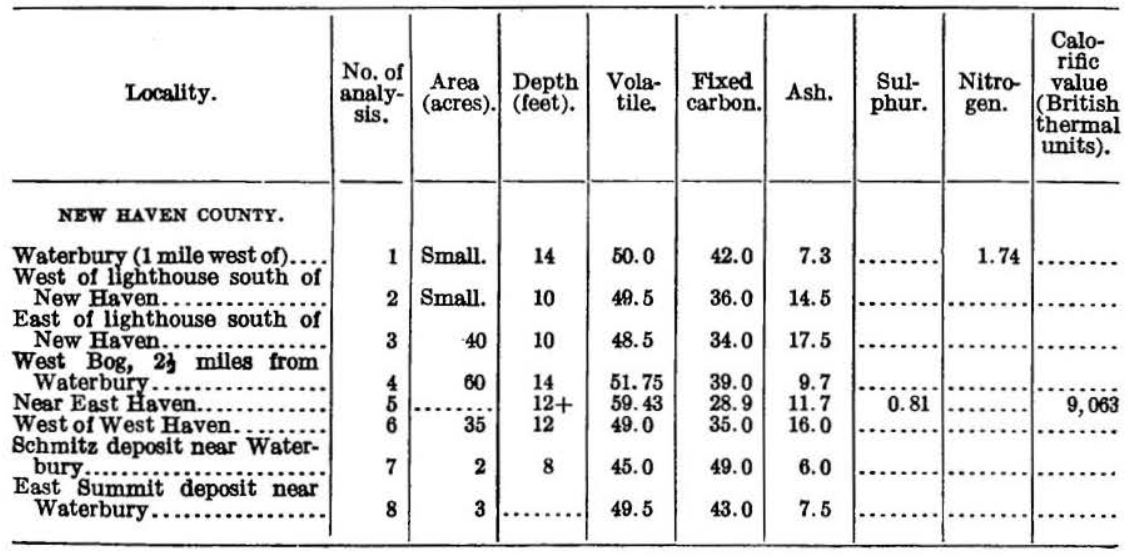


Analyses of peat and muck. $a$

[F. M. Stanton, U. S. Geological Survey, analyst.]

\begin{tabular}{|c|c|c|c|c|c|c|c|c|c|c|c|c|c|c|}
\hline \multirow[b]{2}{*}{ Locality. } & \multirow{2}{*}{$\begin{array}{c}\text { Labora- } \\
\text { tory } \\
\text { No. }\end{array}$} & \multirow{2}{*}{$\begin{array}{c}\text { Condi- } \\
\text { tion of } \\
\text { sam- } \\
\text { ple. }\end{array}$} & \multicolumn{4}{|c|}{ Proximate. } & \multicolumn{5}{|c|}{ Ultimste. } & \multirow{2}{*}{$\begin{array}{l}\text { Air- } \\
\text { drying } \\
\text { loss. }\end{array}$} & \multicolumn{2}{|c|}{ Calorifle value. } \\
\hline & & & $\begin{array}{l}\text { Mois- } \\
\text { ture. }\end{array}$ & $\begin{array}{c}\text { Vola- } \\
\text { tile } \\
\text { matter. }\end{array}$ & $\begin{array}{c}\text { Fixed } \\
\text { carbon. }\end{array}$ & Ash. & $\begin{array}{l}\text { Sul- } \\
\text { phur. }\end{array}$ & $\begin{array}{c}\text { Hydro- } \\
\text { gen. }\end{array}$ & $\begin{array}{l}\text { Car- } \\
\text { bon. }\end{array}$ & $\begin{array}{l}\text { Nitro- } \\
\text { gen. }\end{array}$ & $\begin{array}{l}\text { Oxy- } \\
\text { gen. }\end{array}$ & & $\begin{array}{c}\text { Calo- } \\
\text { ries }\end{array}$ & \begin{tabular}{|c} 
British \\
thermal \\
units.
\end{tabular} \\
\hline \multicolumn{15}{|l|}{ FAIRFIELD COUNTY. } \\
\hline \multirow{3}{*}{ 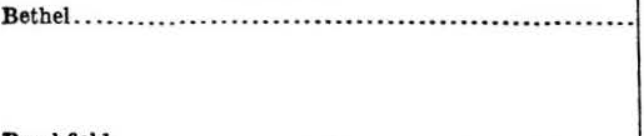 } & 6195 & 1 & 89.03 & $\begin{array}{r}5.98 \\
6.10\end{array}$ & 2.65 & 2. 22 & $: 11$ & & & & & 88.30 & $\begin{array}{r}4,566 \\
481\end{array}$ & $\begin{array}{r}8,219 \\
860\end{array}$ \\
\hline & 6196 & $\begin{array}{l}\mathbf{2} \\
1\end{array}$ & $\dddot{88.24}$ & $\begin{array}{r}55.61 \\
4.30\end{array}$ & $\begin{array}{r}24.15 \\
1.34\end{array}$ & $\begin{array}{r}20.24 \\
6.12\end{array}$ & $\begin{array}{r}1.00 \\
.12\end{array}$ & & & & & $\dddot{87.60}$ & $\begin{array}{r}4,385 \\
300\end{array}$ & $\begin{array}{r}7,893 \\
540\end{array}$ \\
\hline & 6182 & $\begin{array}{l}2 \\
1\end{array}$ & 59.0i & $\begin{array}{l}36.56 \\
24.71\end{array}$ & $\begin{array}{l}11.40 \\
12.25\end{array}$ & $\begin{array}{r}52.04 \\
4.03\end{array}$ & $\begin{array}{r}1.02 \\
.30\end{array}$ & $(\cdots \cdots \cdots \cdot$ & & & & & 2,551 & $\begin{array}{l}4,592 \\
3,681\end{array}$ \\
\hline \multirow{2}{*}{ 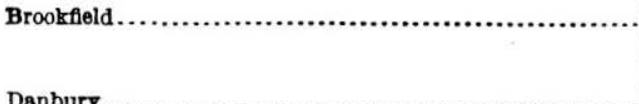 } & 6183 & $\begin{array}{l}2 \\
1\end{array}$ & $\because 31.43$ & $\begin{array}{l}60.28 \\
27.27\end{array}$ & $\begin{array}{l}29.89 \\
11.06\end{array}$ & $\begin{array}{r}9.83 \\
10.24\end{array}$ & $\begin{array}{l}.73 \\
.53\end{array}$ & $\cdots \cdots \cdot$ & & & . & $\dddot{40 \%}$ & $\begin{array}{l}4,989 \\
2,121\end{array}$ & $\begin{array}{l}8,980 \\
3,818\end{array}$ \\
\hline & & 2 & $0 \times 1.20$ & 56.15 & 22.77 & 21. 08 & 1.09 & & & & & 20.70 & 4,367 & 7,861 \\
\hline \multirow{2}{*}{ 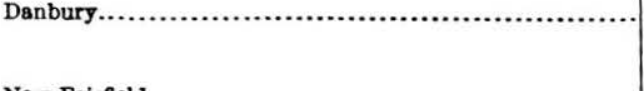 } & 6188 & $\begin{array}{l}1 \\
2\end{array}$ & 93.83 & $\begin{array}{r}3.79 \\
61.43\end{array}$ & $\begin{array}{r}1.87 \\
30.30\end{array}$ & 8.27 & $\begin{array}{l}.03 \\
.49\end{array}$ & $\cdots \cdots$ & $\cdots \cdots$ & $\cdots$ & $\cdots . .$. & 93.30 & $\begin{array}{r}302 \\
4.895\end{array}$ & 8,811 \\
\hline & 6189 & 1 & 90.31 & 3.79 & 1.27 & 4. 63 & .08 & $\cdots$ & .......... & $\ldots$ & ….... & 89.10 & 284 & 511 \\
\hline \multirow{8}{*}{ New Fairfeld } & 6203 & $\begin{array}{l}2 \\
1\end{array}$ & 9.63 & $\begin{array}{l}39.11 \\
54.65\end{array}$ & $\begin{array}{l}13.11 \\
27.79\end{array}$ & $\begin{array}{r}47.78 \\
7.93\end{array}$ & $\begin{array}{l}.83 \\
.46\end{array}$ & $\cdots$ & & & ……... & .00 & $\begin{array}{l}2,931 \\
4,367\end{array}$ & $\begin{array}{l}5,276 \\
7,861\end{array}$ \\
\hline & 6204 & $\begin{array}{l}2 \\
1\end{array}$ & 9.42 & $\begin{array}{l}60.47 \\
46.23\end{array}$ & $\begin{array}{l}30.75 \\
23.87\end{array}$ & $\begin{array}{r}8.78 \\
20.48\end{array}$ & $\begin{array}{r}.51 \\
.38\end{array}$ & & & & & $\cdots: 00$ & $\begin{array}{l}4,832 \\
3,819\end{array}$ & $\begin{array}{l}8,698 \\
6,874\end{array}$ \\
\hline & 6205 & $\begin{array}{l}\tilde{2} \\
1\end{array}$ & $\dddot{24} 90$ & $\begin{array}{l}51.04 \\
42.27\end{array}$ & $\begin{array}{l}26.35 \\
18.93\end{array}$ & $\begin{array}{l}22.61 \\
13.90\end{array}$ & $\begin{array}{r}.42 \\
.84\end{array}$ & & & & …......... & ig 20 & $\begin{array}{l}4,216 \\
3,542\end{array}$ & $\begin{array}{l}7,589 \\
6,376\end{array}$ \\
\hline & & 2 & & 56. 28 & 25.21 & 18. 51 & 1.12 & ……... & & & & & 4,716 & 8,489 \\
\hline & 6200 & $\begin{array}{l}1 \\
2\end{array}$ & 87.90 & $\begin{array}{r}7.24 \\
59.83\end{array}$ & $\begin{array}{r}4.02 \\
33.23\end{array}$ & $\begin{array}{r}.84 \\
6.94\end{array}$ & $\begin{array}{r}.04 \\
33\end{array}$ & $\ldots \ldots \ldots$ & .......... & ..... & ............. & 87.10 & $\begin{array}{r}600 \\
4\end{array}$ & 1,080 \\
\hline & 6201 & 1 & 91.23 & 5.15 & 2. 98 & $\therefore 64$ & .03 & ……... & ......... & $\cdots \cdots$ & ....... & 90.80 & 421 & \\
\hline & 6202 & $\begin{array}{l}2 \\
1\end{array}$ & $\dddot{82} 115$ & $\begin{array}{l}58.72 \\
11.52\end{array}$ & $\begin{array}{r}33.98 \\
5.49\end{array}$ & $\begin{array}{r}7.30 \\
.84\end{array}$ & $\begin{array}{l}.34 \\
.08\end{array}$ & & & & …... & 81.10 & $\begin{array}{r}4,800 \\
909\end{array}$ & $\begin{array}{l}8,640 \\
1,638\end{array}$ \\
\hline & 6190 & $\begin{array}{l}2 \\
1\end{array}$ & $\cdots 9.32$ & $\begin{array}{l}64.54 \\
22.47\end{array}$ & $\begin{array}{r}30.75 \\
9.02\end{array}$ & $\begin{array}{r}4.71 \\
59.19\end{array}$ & $\begin{array}{r}.45 \\
.66\end{array}$ & …........ & …….... & ……..... & …....... & 5.00 & $\begin{array}{l}5,092 \\
1,725\end{array}$ & $\begin{array}{l}9,166 \\
3,105\end{array}$ \\
\hline \multirow{5}{*}{ 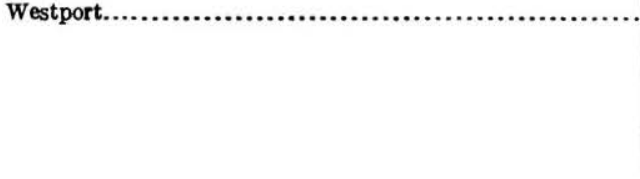 } & & 2 & . & 24.78 & 9.95 & 65.27 & .73 & ........... & 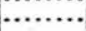 & ……… & 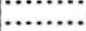 & 0.00 & 1,902 & $.3,424$ \\
\hline & 6191 & $\begin{array}{l}1 \\
2\end{array}$ & $\begin{array}{r}12.70 \\
\end{array}$ & $\begin{array}{l}55.61 \\
63.70\end{array}$ & $\begin{array}{l}27.57 \\
31.58\end{array}$ & $\begin{array}{l}4.12 \\
4.72\end{array}$ & .24 & $\cdots \ldots \ldots$ & . $\cdots \cdots \cdots$ & ............. & …........ & 5.80 & $\begin{array}{l}4,772 \\
5,466\end{array}$ & $\begin{array}{l}8,590 \\
9.839\end{array}$ \\
\hline & 6192 & & 5.58 & 32.14 & 10. 75 & 51.53 & .40 & …......... & …....... & ........ & (1) & .00 & 2,361 & 4,250 \\
\hline & 6198 & \begin{tabular}{l|l}
2 \\
1
\end{tabular} & 19.69 & $\begin{array}{l}34.04 \\
48.83\end{array}$ & $\begin{array}{l}11.38 \\
28.25\end{array}$ & $\begin{array}{r}54.58 \\
3.23\end{array}$ & $\begin{array}{r}.42 \\
.19\end{array}$ & …........ & …, & ……. & ㅈ..…" & ii.o0 & $\begin{array}{l}2,501 \\
4,273\end{array}$ & $\begin{array}{l}4,502 \\
7,691\end{array}$ \\
\hline & & 21 & & 60.80 & 35.18 & 4.02 & .24 & & & & & & 5,321 & 9,578 \\
\hline
\end{tabular}




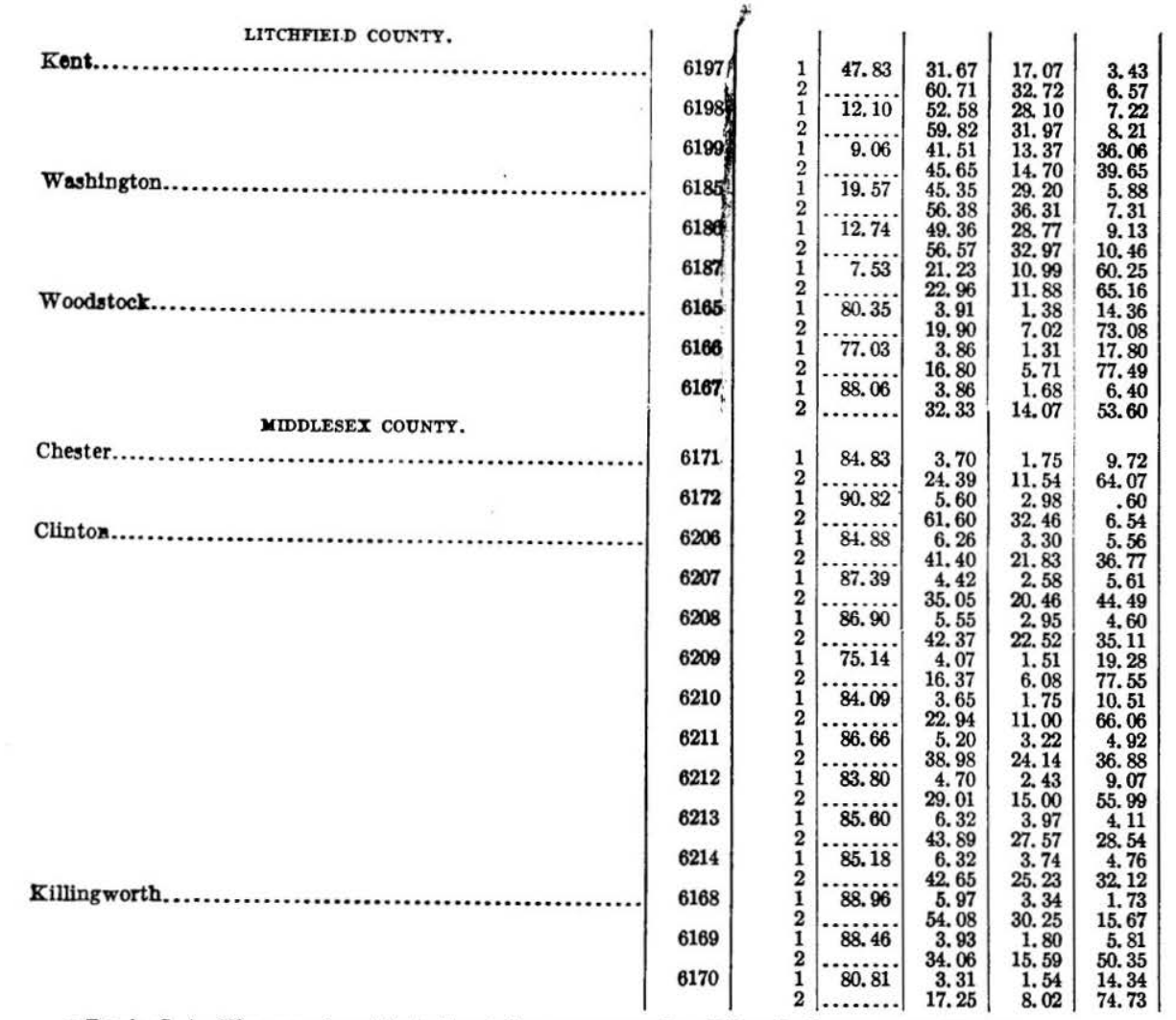

a Davis, C. A., The uses of peat for fuel and other purposes: Bur. Mines Bull. 16,pp 186-189,1911.

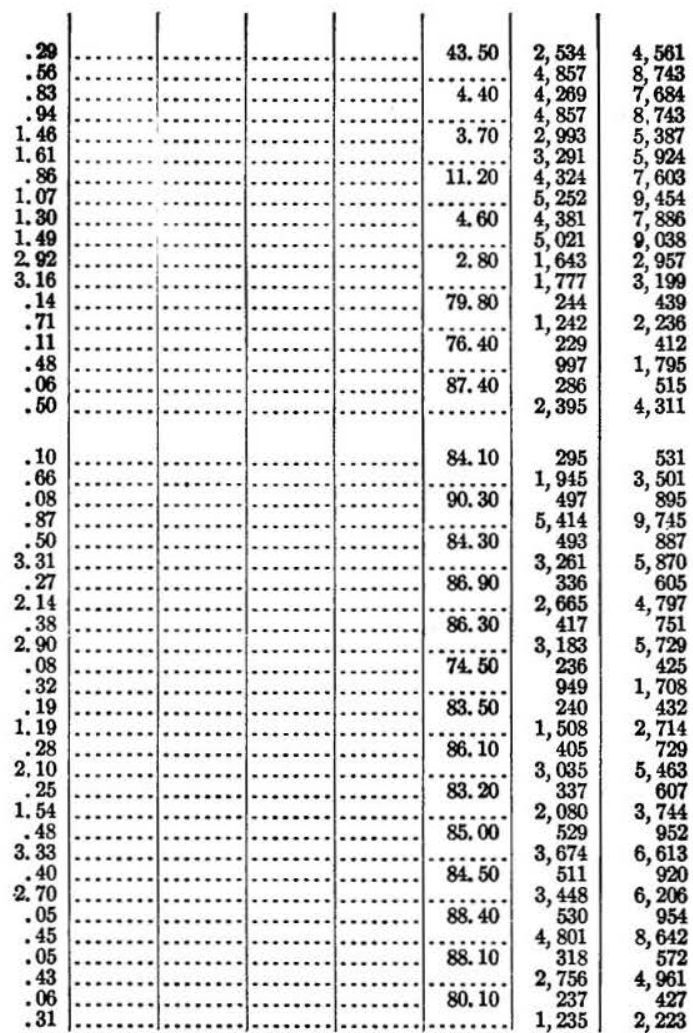

b 1, Native peat. 2, Moisture-free peat. 
Analyses of peat and muck-Continued.

\begin{tabular}{|c|c|c|c|c|c|c|c|c|c|c|c|c|c|c|}
\hline \multirow[b]{2}{*}{ Locality. } & \multirow{2}{*}{$\begin{array}{c}\text { Labora- } \\
\text { tory } \\
\text { No. }\end{array}$} & \multirow{2}{*}{$\begin{array}{l}\text { Condi- } \\
\text { tion of } \\
\text { sam- } \\
\text { ple. }\end{array}$} & \multicolumn{4}{|c|}{ Proximate. } & \multicolumn{5}{|c|}{ Ultimate. } & \multirow[b]{2}{*}{$\begin{array}{l}\text { Air- } \\
\text { drying } \\
\text { loss. }\end{array}$} & \multicolumn{2}{|c|}{ Calorific value. } \\
\hline & & & $\begin{array}{l}\text { Mois- } \\
\text { ture. }\end{array}$ & $\begin{array}{c}\text { Vola- } \\
\text { tile } \\
\text { matter. }\end{array}$ & $\begin{array}{l}\text { Fixed } \\
\text { carbon. }\end{array}$ & Ash. & $\begin{array}{l}\text { Sul- } \\
\text { phur. }\end{array}$ & $\begin{array}{c}\text { Hydro- } \\
\text { gen. }\end{array}$ & $\begin{array}{l}\text { Car- } \\
\text { bon. }\end{array}$ & $\begin{array}{l}\text { Nitro- } \\
\text { gen. }\end{array}$ & $\begin{array}{l}\text { Oxy- } \\
\text { gen. }\end{array}$ & & $\begin{array}{l}\text { Calo- } \\
\text { ries. }\end{array}$ & $\begin{array}{c}\text { British } \\
\text { thermal } \\
\text { units. }\end{array}$ \\
\hline NEW HAVEN COUNTY. & & & & & & & . & & & & & & & \\
\hline \multirow{2}{*}{ 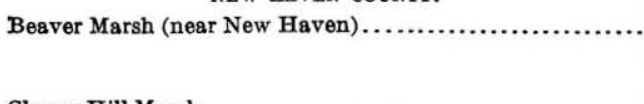 } & 6155 & $\frac{1}{2}$ & 91.20 & $\begin{array}{r}6.61 \\
75.11\end{array}$ & $\begin{array}{r}1.84 \\
20.91\end{array}$ & $\begin{array}{l}0.35 \\
3.98\end{array}$ & 0.02 & $\begin{array}{r}10.59 \\
5.23\end{array}$ & $\begin{array}{r}5.00 \\
56.82\end{array}$ & 0.13 & $\begin{array}{l}83.91 \\
32.26\end{array}$ & 90.10 & $\begin{array}{r}472 \\
364\end{array}$ & 850 \\
\hline & 6156 & 1 & 93.10 & 4.14 & 2.13 & .62 & .03 & 10.70 & 3.69 & .15 & 84.80 & 92.30 & 0,304 & $\begin{array}{r}9,655 \\
637\end{array}$ \\
\hline Cherry Hill Marsh........ & 6157 & 1 & $\dddot{86.98}$ & $\begin{array}{r}60.00 \\
4.91\end{array}$ & 2.21 & 9. 90 & $\begin{array}{r}.43 \\
.14\end{array}$ & $\begin{array}{r}5.22 \\
10.12\end{array}$ & $\begin{array}{r}53.48 \\
4.13\end{array}$ & $\begin{array}{r}2.17 \\
.25\end{array}$ & $\begin{array}{l}29.57 \\
79.46\end{array}$ & 85.70 & $\begin{array}{r}5,130 \\
408\end{array}$ & $\begin{array}{r}9,234 \\
734\end{array}$ \\
\hline \multirow{5}{*}{ 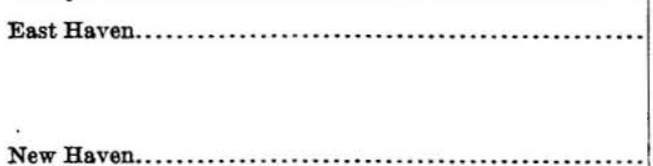 } & 6159 & 1 & 87.41 & $\begin{array}{r}37.71 \\
6.66\end{array}$ & $\begin{array}{r}16.98 \\
4.06\end{array}$ & $\begin{array}{r}45.31 \\
1.87\end{array}$ & $\begin{array}{r}1.08 \\
.15\end{array}$ & 3.53 & 31.72 & 1.92 & $\begin{array}{c}16.44 \\
\ldots \ldots \ldots\end{array}$ & 78.00 & $\begin{array}{r}3,134 \\
602\end{array}$ & $\begin{array}{l}5,641 \\
1,084\end{array}$ \\
\hline & 6160 & $\begin{array}{l}2 \\
1\end{array}$ & $\begin{array}{l}52.90 \\
90.09\end{array}$ & $\begin{array}{r}32.25 \\
5.89\end{array}$ & $\begin{array}{r}14.85 \\
2.86\end{array}$ & $\begin{array}{l}1.19 \\
1.16\end{array}$ & .08 & $\cdot$ & & & & 89.20 & $\begin{array}{r}4,782 \\
499\end{array}$ & $\begin{array}{r}8,608 \\
898\end{array}$ \\
\hline & & $\begin{array}{l}2 \\
1\end{array}$ & 84.69 & $\begin{array}{r}59.43 \\
4.66\end{array}$ & $\begin{array}{r}28.86 \\
1.63\end{array}$ & $\begin{array}{r}11.71 \\
9.02\end{array}$ & $\begin{array}{l}.81 \\
.28\end{array}$ & & & & & $\because 30$ & 5,035 & 9,063 \\
\hline & 6161 & $\frac{1}{2}$ & 84.69 & $\begin{array}{r}4.66 \\
30.44\end{array}$ & $\begin{array}{r}1.63 \\
10.64\end{array}$ & $\begin{array}{r}9.02 \\
58.92\end{array}$ & 1. 83 & & & & & 83.80 & $\begin{array}{r}351 \\
2,293\end{array}$ & $\begin{array}{r}632 \\
4,127\end{array}$ \\
\hline & 6158 & $\begin{array}{l}1 \\
2\end{array}$ & 83.91 & $\begin{array}{r}4.88 \\
30.33\end{array}$ & $\begin{array}{r}2.76 \\
17.15\end{array}$ & $\begin{array}{r}8.45 \\
52.52\end{array}$ & $\begin{array}{r}.41 \\
2.55\end{array}$ & & & & …….... & 83.40 & $\begin{array}{r}390 \\
2,424\end{array}$ & $\begin{array}{r}702 \\
4.363\end{array}$ \\
\hline New Haven................................................... & 6184 & $\begin{array}{l}1 \\
2\end{array}$ & 87.77 & $\begin{array}{r}4.36 \\
35.65\end{array}$ & $\begin{array}{r}2.54 \\
20.77\end{array}$ & $\begin{array}{r}5.33 \\
43.58\end{array}$ & $\begin{array}{r}.37 \\
3.03\end{array}$ & …. & ……... & a.......... & a........... & 87.50 & $\begin{array}{l}2,424 \\
296\end{array}$ & $\begin{array}{r}4,305 \\
533 \\
4,56\end{array}$ \\
\hline \multirow{8}{*}{ 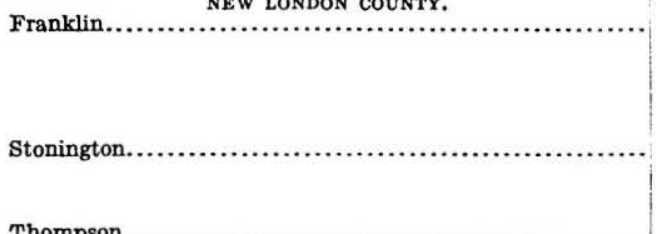 } & & & …....... & & & & & & & & …... & ............ & & \\
\hline & 6173 & $\mathbf{1}^{\prime}$ & 92.98 & $\begin{array}{r}4.29 \\
.61 .11\end{array}$ & $\begin{array}{r}2.31 \\
32.91\end{array}$ & $\begin{array}{r}.42 \\
5.98\end{array}$ & $\begin{array}{l}.03 \\
.43\end{array}$ & & & & & 92.50 & $\begin{array}{r}361 \\
5,142\end{array}$ & $\begin{array}{r}650 \\
9.256\end{array}$ \\
\hline & 6174 & $\begin{array}{l}1 \\
2\end{array}$ & 87.82 & $\begin{array}{r}5.16 \\
42.36\end{array}$ & $\begin{array}{r}1.59 \\
13.06\end{array}$ & $\begin{array}{r}5.43 \\
44.58\end{array}$ & .08 & ….... & .......... & ......... & {$[\cdots . . . \cdots . .$.} & 87.20 & 370 & 666 \\
\hline & 6175 & 1 & 87.23 & 4.13 & $\begin{array}{r}10.00 \\
2.12\end{array}$ & 6.52 & $\begin{array}{r}.00 \\
.15 \\
\end{array}$ & & & & ?... & 86.40 & $\begin{array}{r}3,458 \\
338\end{array}$ & $\begin{array}{l}0,4008 \\
608\end{array}$ \\
\hline & 6180 & & $\dddot{88}$ & $\begin{array}{r}32.34 \\
6.03\end{array}$ & $\begin{array}{r}16.60 \\
3.00\end{array}$ & $\begin{array}{r}51.06 \\
2.42\end{array}$ & $\begin{array}{r}1.17 \\
.09\end{array}$ & & & & $\cdots$ & $\because 7$ & 2,647 & 4,765 \\
\hline & 6180 & $\begin{array}{l}1 \\
2\end{array}$ & $\begin{array}{r}88.55 \\
\ldots .6 .\end{array}$ & $\begin{array}{r}6.03 \\
52.66\end{array}$ & $\begin{array}{r}3.00 \\
26.20\end{array}$ & $\begin{array}{r}2.42 \\
21.14\end{array}$ & $\begin{array}{l}.09 \\
.79\end{array}$ & ........ & …' & . & ….... & 87.90 & $\begin{array}{r}523 \\
4,568\end{array}$ & $\begin{array}{r}941 \\
8,222\end{array}$ \\
\hline & 6181 & 1 & 87.87 & 7. 42 & 3. 83 & .88 & .05 & $\ldots$. & ........... & …….... & …......... & 87.20 & 674 & 1,213 \\
\hline & 6162 & $\frac{2}{1}$ & 9225 & $\begin{array}{r}61.17 \\
4.51\end{array}$ & $\begin{array}{r}31.58 \\
2.52\end{array}$ & $\begin{array}{r}7.25 \\
.72\end{array}$ & $\begin{array}{r}.41 \\
.03\end{array}$ & $\mid \cdots \cdots \cdots$ & ………. & …........ & a......... & 91.60 & $\begin{array}{r}5,556 \\
394\end{array}$ & $\begin{array}{r}10,001 \\
709\end{array}$ \\
\hline \multirow{9}{*}{ 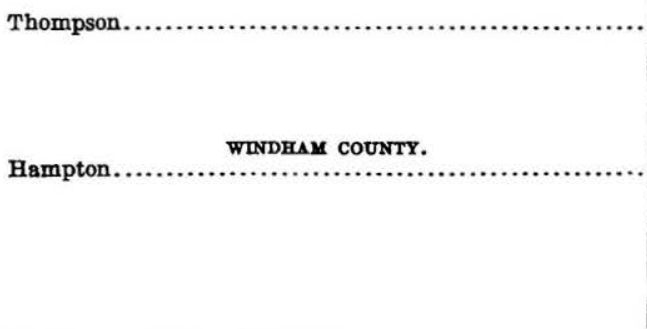 } & 6163 & ${ }_{1}^{2}$ & 85.66 & $\begin{array}{r}58.19 \\
8.52\end{array}$ & $\begin{array}{r}32.52 \\
4.54\end{array}$ & $\begin{array}{l}9.29 \\
1.28\end{array}$ & $\begin{array}{r}1.03 \\
.10\end{array}$ & & 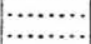 & 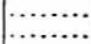 & 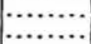 & 85.00 & $\begin{array}{r}5,084 \\
768\end{array}$ & $\begin{array}{l}9,151 \\
1,382\end{array}$ \\
\hline & 6164 & $\begin{array}{l}2 \\
1\end{array}$ & 88.64 & $\begin{array}{r}59.41 \\
4.27\end{array}$ & $\begin{array}{r}31.66 \\
2.00\end{array}$ & $\begin{array}{l}8.93 \\
5.09\end{array}$ & .70 & & …....... & $\ldots \ldots \ldots$ & …........ & $\dddot{88.30}$ & $\begin{array}{r}5,356 \\
336\end{array}$ & 9,641 \\
\hline & & 2 & & 37.59 & 17.60 & 44.81 & .96 & & & $\cdots \cdots \cdots$ & $\cdots \cdots \cdots$ & & 2,958 & 5,324 \\
\hline & & 1 & 88.52 & 3.33 & 1.20 & 6.95 & .05 & & & & & 87.80 & 245 & 441 \\
\hline & 6178 & $\begin{array}{l}2 \\
1\end{array}$ & 91.10 & $\begin{array}{r}29.01 \\
5.19\end{array}$ & $\begin{array}{r}10.45 \\
2.99\end{array}$ & $\begin{array}{r}60.54 \\
.72\end{array}$ & .07 & r.s.ina. & ........ & .... & ;......... & 90,40 & $\begin{array}{r}2,134 \\
446\end{array}$ & $\begin{array}{l}3,841 \\
803\end{array}$ \\
\hline & 6177 & $\tilde{2}$ & (1) & 58. 31 & 33. 60 & 8.09 & .79 & …….... & …........ & $\cdots \cdots$ & …....... & & 5,011 & 9,020 \\
\hline & 6178 & & 91.60 & $\begin{array}{r}4.95 \\
58.93\end{array}$ & $\begin{array}{r}2.78 \\
33.09\end{array}$ & $\begin{array}{r}.67 \\
7.98\end{array}$ & $: .06$ & …..... & …..... & …...... & …...... & 80.90 & 5.095 & 9771 \\
\hline & & & 91.02 & 5. 37 & 3. 00 & .61 & .11 & ........... & :.......... & .............. & '............. & 90.30 & 455 & 819 \\
\hline & 6179 & 2 & & 59.80 & 33.41 & 6.79 & 1.22 & $\mid . . . . .$. & ......... & .......... & ......... & & 5,067 & 9,121 \\
\hline
\end{tabular}


FLORIDA.

Analyses of peat and muck. $a$

象

[F. M. Stanton, U. S. Geological Survey, analyst.]

\begin{tabular}{|c|c|c|c|c|c|c|c|c|c|c|c|c|c|c|}
\hline \multirow[b]{2}{*}{ Locality. } & \multirow{2}{*}{$\begin{array}{c}\text { Labora- } \\
\text { tory. } \\
\text { No. }\end{array}$} & \multirow{2}{*}{$\mid \begin{array}{c}\text { Condi- } \\
\text { tion of } \\
\text { sam- } \\
\text { ple.b }\end{array}$} & \multicolumn{4}{|c|}{ Proximate. } & \multicolumn{5}{|c|}{ - Ultimate. } & \multirow{2}{*}{$\begin{array}{l}\text { Air- } \\
\text { drying } \\
\text { loss. }\end{array}$} & \multicolumn{2}{|c|}{ Calorific value. } \\
\hline & & & $\begin{array}{l}\text { Mois- } \\
\text { ture. }\end{array}$ & $\begin{array}{c}\text { Volar } \\
\text { tile } \\
\text { matter. }\end{array}$ & $\begin{array}{c}\text { Fixed } \\
\text { carbon. }\end{array}$ & Ash. & $\begin{array}{c}\text { Sul- } \\
\text { phur. }\end{array}$ & $\begin{array}{c}\text { Hydro- } \\
\text { gen. }\end{array}$ & $\begin{array}{l}\text { Car- } \\
\text { bon. }\end{array}$ & $\begin{array}{l}\text { Nitro- } \\
\text { gen. }\end{array}$ & $\begin{array}{l}\text { Oxy- } \\
\text { gen. }\end{array}$ & & $\begin{array}{l}\text { Calo- } \\
\text { ries. }\end{array}$ & $\begin{array}{c}\text { British } \\
\text { thermal } \\
\text { units. }\end{array}$ \\
\hline CTRRUS COUNTY. & & & & & & & & & & & & & & \\
\hline Inverness, half a mile northeast of (T.19 S., R. 20 E.) .......... & $\begin{array}{l}\text { c } 7205 \\
\text { d } 7204\end{array}$ & $\begin{array}{l}1 \\
1 \\
2\end{array}$ & $\begin{array}{r}89.49 \\
28.14 \\
\cdots\end{array}$ & 60.17 & 27.46 & $\begin{array}{r}8.89 \\
12.37\end{array}$ & $\begin{array}{l}0.72 \\
1.00\end{array}$ & & & & & $\begin{array}{r}88.60 \\
21.40 \\
\cdots . . . . . .\end{array}$ & $\begin{array}{l}3,725 \\
5,184\end{array}$ & 90,7305 \\
\hline 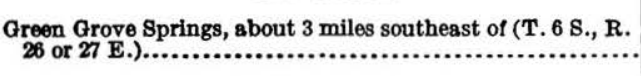 & $d 7106$ & 1 & 9. 59 & 6.31 & .67 & 83.43 & .08 & & & 0.12 & & 6.50 & & ........... \\
\hline DADE COUNTY. & & & $\cdots \cdots$ & & & & & & & & & & & \\
\hline Everglades, south edge of...................... & d 7560 & $\begin{array}{l}1 \\
2\end{array}$ & $\begin{array}{c}41.92 \\
\ldots \ldots . . .\end{array}$ & $\begin{array}{r}6.63 \\
11.42\end{array}$ & $\begin{array}{l}22.38 \\
38.53\end{array}$ & $\begin{array}{l}29.07 \\
50.05\end{array}$ & .05 & & & & & 40.90 & $\begin{array}{l}388 \\
668\end{array}$ & $\begin{array}{r}698 \\
1,202\end{array}$ \\
\hline Fort Lauderdale, about 10 miles west of...... & $\begin{array}{l}\text { c } 7638 \\
\text { d } 7637\end{array}$ & $\begin{array}{l}1 \\
1 \\
2\end{array}$ & 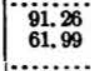 & $\begin{array}{l}20.010 \\
52.88\end{array}$ & $\because i 1 . \%$ & $\begin{array}{r}6.04 \\
15.89\end{array}$ & .16 & …...... & (a.......... & (n......... & $\cdots \cdots \cdots \cdot$ & $\begin{array}{l}90.40 \\
57.90 \\
\ldots \ldots\end{array}$ & $\begin{array}{r}7,7,746 \\
4,594\end{array}$ & $\begin{array}{l}3,143 \\
8,269\end{array}$ \\
\hline Miami, $1 \frac{1}{1}$ miles northwest of (sec. 35, T. 53 S., R. 41 E.)........ & $\begin{array}{l}\text { c } 7604 \\
\text { d } 7603\end{array}$ & $\begin{array}{l}1 \\
1 \\
2\end{array}$ & $\begin{array}{l}92.75 \\
66.59\end{array}$ & ii. 52 & 26.01 & 39.20 & 2.66 & & & $\cdots \cdots$ & 64.30 & i..90 & $\mid \begin{array}{ll}n+\cdots \\
\cdots \cdots \cdots\end{array}$ & a....... \\
\hline Miami, 5 miles west of (T. 53 S., R. 41 E.)........... & $\begin{array}{l}\text { c } 7802 \\
\text { d } 7601\end{array}$ & $\begin{array}{l}1 \\
1 \\
2\end{array}$ & $\begin{array}{l}91.63 \\
76.31 \\
\ldots . . .\end{array}$ & $\because$ ii. & $\begin{aligned} 7.13 \\
34.46\end{aligned}$ & "i. 1.91 & $\frac{.13}{.63}$ & & & (n........ & (n.......... & $\begin{array}{r}90.90 \\
77.40 \\
\cdots .7 .7\end{array}$ & i,, 384 & $\begin{array}{l}2,00 j \\
9,691\end{array}$ \\
\hline $\begin{array}{l}\text { Paradise Key, northwest of................................... } \\
\text { DE soTo counTY. }\end{array}$ & с 7559 & $\overline{1}$ & 89.41 & $\cdots \cdots$ & …...... & & & & & & $\cdots \cdots$ & 88.70 & $\cdots \cdots \cdots$ & \\
\hline $\begin{array}{c}\text { Nocatee, west of.......................... } \\
\text { DUVAL counTY. }\end{array}$ & $\begin{array}{l}\text { c } 7320 \\
\text { d } 7321\end{array}$ & $\begin{array}{l}1 \\
1 \\
2\end{array}$ & $\begin{array}{r}87.99 \\
14.17 \\
\cdots \cdots\end{array}$ & $\begin{array}{l}51.32 \\
59.79\end{array}$ & 30.50 & 9. 33 & .31 & & & ….... & ;........ & $\begin{array}{r}87.20 \\
5.90 \\
2 . \cdots\end{array}$ & $\begin{array}{r}4,489 \\
5,230\end{array}$ & $\dddot{8}, \dddot{0} 0 \ddot{0}$ \\
\hline 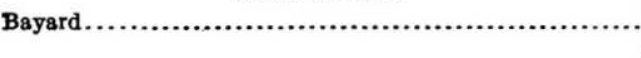 & 6028 & $\begin{array}{l}1 \\
2\end{array}$ & $\begin{aligned} 73.10 \\
. .2 \ldots . . .\end{aligned}$ & $\begin{array}{l}14.00 \\
52.04\end{array}$ & $\begin{array}{r}8.05 \\
29.93\end{array}$ & $\begin{array}{r}4.85 \\
18.03\end{array}$ & $\begin{array}{l}1.06 \\
3.94\end{array}$ & $\begin{array}{l}9.27 \\
4.28\end{array}$ & $\begin{array}{l}13.37 \\
49.70\end{array}$ & $\begin{array}{r}.53 \\
1.97\end{array}$ & $\begin{array}{l}70.92 \\
22.08\end{array}$ & $\begin{array}{l}69.90 \\
\cdots \cdots . . .\end{array}$ & \begin{tabular}{|l|}
1,283 \\
4,770
\end{tabular} & $\begin{array}{l}2,309 \\
8,586\end{array}$ \\
\hline
\end{tabular}


Analyses of peat and muck-Continued.

\begin{tabular}{|c|c|c|c|c|c|c|c|c|c|c|c|c|c|c|}
\hline \multirow[b]{2}{*}{ c } & \multirow{2}{*}{\begin{tabular}{|c} 
Labora- \\
tory \\
No.
\end{tabular}} & \multirow{2}{*}{$\begin{array}{c}\text { Condi- } \\
\text { tion of } \\
\text { sam- } \\
\text { ple. }\end{array}$} & \multicolumn{4}{|c|}{ Proximate. } & \multicolumn{5}{|c|}{ Ultimate. } & \multirow{2}{*}{$\begin{array}{l}\text { Air- } \\
\text { drying } \\
\text { loss. }\end{array}$} & \multicolumn{2}{|c|}{ Calorifie value. } \\
\hline & & & $\begin{array}{l}\text { Mois- } \\
\text { ture. }\end{array}$ & $\begin{array}{c}\text { Vola- } \\
\text { tile } \\
\text { matter. }\end{array}$ & $\begin{array}{l}\text { Fixed } \\
\text { carbon. }\end{array}$ & Ash. & $\begin{array}{c}\text { Sul- } \\
\text { phur. }\end{array}$ & $\begin{array}{c}\text { Hydro- } \\
\text { gen. }\end{array}$ & $\begin{array}{l}\text { Car- } \\
\text { bon. }\end{array}$ & $\begin{array}{l}\text { Nitro- } \\
\text { gen. }\end{array}$ & $\begin{array}{l}\text { Oxy- } \\
\text { gen. }\end{array}$ & & $\begin{array}{l}\text { Calo- } \\
\text { ries. }\end{array}$ & \begin{tabular}{|} 
British \\
thermma \\
units.s.
\end{tabular} \\
\hline 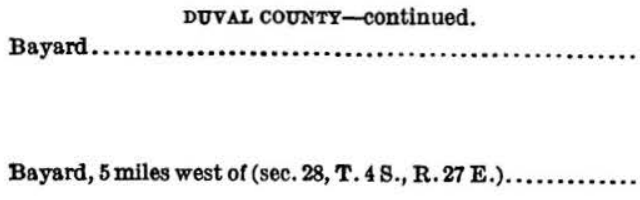 & $\begin{array}{r}6027 \\
6030 \\
6029 \\
a 7115 \\
b 7114\end{array}$ & $\begin{array}{l}1 \\
2 \\
1 \\
2 \\
1 \\
2 \\
1\end{array}$ & \begin{tabular}{c}
73.56 \\
775.20 \\
\hdashline $7 i .14$ \\
\hdashline$\% 9.27$
\end{tabular} & \begin{tabular}{l}
14.44 \\
54.61 \\
13.00 \\
52.61 \\
13.98 \\
48.44 \\
\hdashline \\
.7 .7$.
\end{tabular} & $\begin{array}{r}8.11 \\
30.68 \\
7.60 \\
30.76 \\
7.47 \\
25.88 \\
\hdashline 7.9\end{array}$ & $\begin{array}{r}3.89 \\
14.71 \\
4.11 \\
17.63 \\
7.41 \\
25.68 \\
\cdots \\
\cdots\end{array}$ & $\begin{array}{l}1.08 \\
4.08 \\
1.02 \\
4.13 \\
1.05 \\
3.64\end{array}$ & $\begin{array}{l}9.34 \\
4.43 \\
8.42 \\
4.25 \\
8.96 \\
3.67\end{array}$ & $\begin{array}{l}13.84 \\
52.34 \\
12.70 \\
51.40 \\
13.21 \\
45.77\end{array}$ & $\begin{array}{r}0.51 \\
1.93 \\
-48 \\
1.94 \\
1.48 \\
1.66\end{array}$ & $\begin{array}{l}71.34 \\
22.51 \\
72.27 \\
21.65 \\
68.89 \\
19.58\end{array}$ & $\begin{array}{r}67.40 \\
770.80 \\
\hdashline 30.20\end{array}$ & $\begin{array}{l}1,295 \\
4,898 \\
1,195 \\
4,836 \\
1,248 \\
4,324\end{array}$ & $\begin{array}{l}2,331 \\
8,816 \\
2,151 \\
8,705 \\
2,246 \\
7,785 \\
.\end{array}$ \\
\hline HERNANDO COUNTY. & $\begin{array}{l}b 7114 \\
b 7104\end{array}$ & $\begin{array}{l}1 \\
2 \\
1 \\
2\end{array}$ & $\begin{array}{r}50.18 \\
\cdots 34.82 \\
\cdots \cdots\end{array}$ & $\begin{array}{l}27.10 \\
54.41 \\
36.63 \\
56.20\end{array}$ & $\begin{array}{l}15.86 \\
31.84 \\
21.11 \\
32.39\end{array}$ & $\begin{array}{r}6.85 \\
13.75 \\
7.44 \\
11.41\end{array}$ & $\begin{array}{l}1.38 \\
2.77 \\
2.04 \\
3.13\end{array}$ & |c...... & 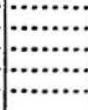 & $\begin{array}{r}1.94 \\
1.89 \\
1.69 \\
2.59\end{array}$ & 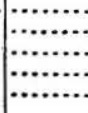 & $\begin{array}{r}89.00 \\
45.50 \\
-28.00\end{array}$ & $\begin{array}{r}3,517 \\
5,053 \\
3,279 \\
5,031\end{array}$ & $\begin{array}{l}4,531 \\
9,096 \\
5,902 \\
9,056\end{array}$ \\
\hline Brooksville, 2 miles southeast of (T. 22 S., R. 19 E.) ............ & $\cdot \mid \begin{array}{l}a \\
b\end{array} 7262$ & $\begin{array}{l}1 \\
\frac{1}{2}\end{array}$ & $\begin{aligned} 70.04 \\
64.75 \\
\cdots \cdots\end{aligned}$ & $\begin{array}{l}3.30 \\
23.55\end{array}$ & $\begin{array}{ll}4.58 \\
12.98\end{array}$ & $\begin{array}{ll}20.37 \\
63.46\end{array}$ & 7.05 & & a........ & & & $\begin{array}{l}69.10 \\
63.30\end{array}$ & $\begin{array}{l}0.659 \\
1,870\end{array}$ & $1,1,186$ \\
\hline LAKE COUNTY. & b 7202 & $\frac{1}{2}$ & 18.12 & $\begin{array}{l}36.19 \\
56.41\end{array}$ & 28.08 & 15. 72 & $\begin{array}{l}.53 \\
.65\end{array}$ & (…..... & ….... & & ….. & 9.80 & $\begin{array}{l}4,404 \\
5,867\end{array}$ & 70,651 \\
\hline Clermont, 2 miles northwest of (sec. 14, T. 22 S., R. 25 E.).. & - $\begin{array}{l}\text { a } 7633 \\
\text { b } 7632 \\
\text { a } 7635\end{array}$ & $\begin{array}{l}1 \\
1 \\
2\end{array}$ & $\begin{array}{l}95.00 \\
79.68\end{array}$ & $\begin{array}{l}13.77 \\
67.80\end{array}$ & $\begin{array}{l}6.23 \\
30.67\end{array}$ & $1.3 \mathrm{i}$ & $\begin{array}{l}7.08 \\
.39\end{array}$ & & & & & $\begin{array}{l}94.60 \\
78.00\end{array}$ & $\because, 2,226$ & 70,865 \\
\hline Eldorado, 1 mile northeast of (T. 19 S., R. $25 \mathrm{E}$ & & $\begin{array}{l}1 \\
2 \\
1\end{array}$ & 82.12 & $\begin{array}{l}11.75 \\
65.72\end{array}$ & $\begin{array}{r}5.72 \\
31.99\end{array}$ & 2.29 & .05 & & & & & 80.40 & $\because, 070$ & $\begin{aligned} 10,886 \\
10,548\end{aligned}$ \\
\hline & & $\begin{array}{l}1 \\
2 \\
1\end{array}$ & 71.35 & $\begin{array}{l}13.39 \\
43.25\end{array}$ & $\begin{array}{l}5.00 \\
17.45\end{array}$ & 39. 30 & $: .17$ & & & & & 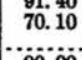 & $\frac{1,0 i i}{3,529}$ & $\begin{aligned} 1,8200 \\
6,352\end{aligned}$ \\
\hline Eustis, 3 miles north of (T. 18 S., R. 26 E.).. & b 7257 & 1 & 72.9 & 66.32 & $\begin{aligned} 7.76 \\
28.63\end{aligned}$ & 5.05 & .08 & & & ....... & & $\begin{array}{ll}71.40 \\
71.0\end{array}$ & $\begin{array}{lll}7,43 i \\
5,279\end{array}$ & $\begin{aligned} 2 \\
2,5756 \\
8,502\end{aligned}$ \\
\hline Leesburg, just east of (T. 19 S., R. 24 E.).. & a 7258 & & \begin{aligned} 5.59 \\
\hdashline 0.0\end{aligned} & 11. 71 & $\begin{array}{l}6.12 \\
6.48\end{array}$ & $\begin{array}{l}77.18 \\
81.75\end{array}$ & .07 & ?........ & (a.......... & 1.85 & & $\begin{aligned} 5.10 \\
3.70\end{aligned}$ & ...... & \\
\hline & b 7259 & & & $\begin{array}{r}7.60 \\
48.69\end{array}$ & $\begin{array}{r}2.57 \\
16.46\end{array}$ & $\begin{array}{r}5.44 \\
34.85\end{array}$ & $\begin{array}{r}.19 \\
1.21\end{array}$ & …..... & $\mid \cdots \ldots .$. & $\begin{array}{r}.41 \\
2.62\end{array}$ & ....... & 83.50 & $\begin{array}{r}587 \\
3,760\end{array}$ & 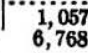 \\
\hline
\end{tabular}




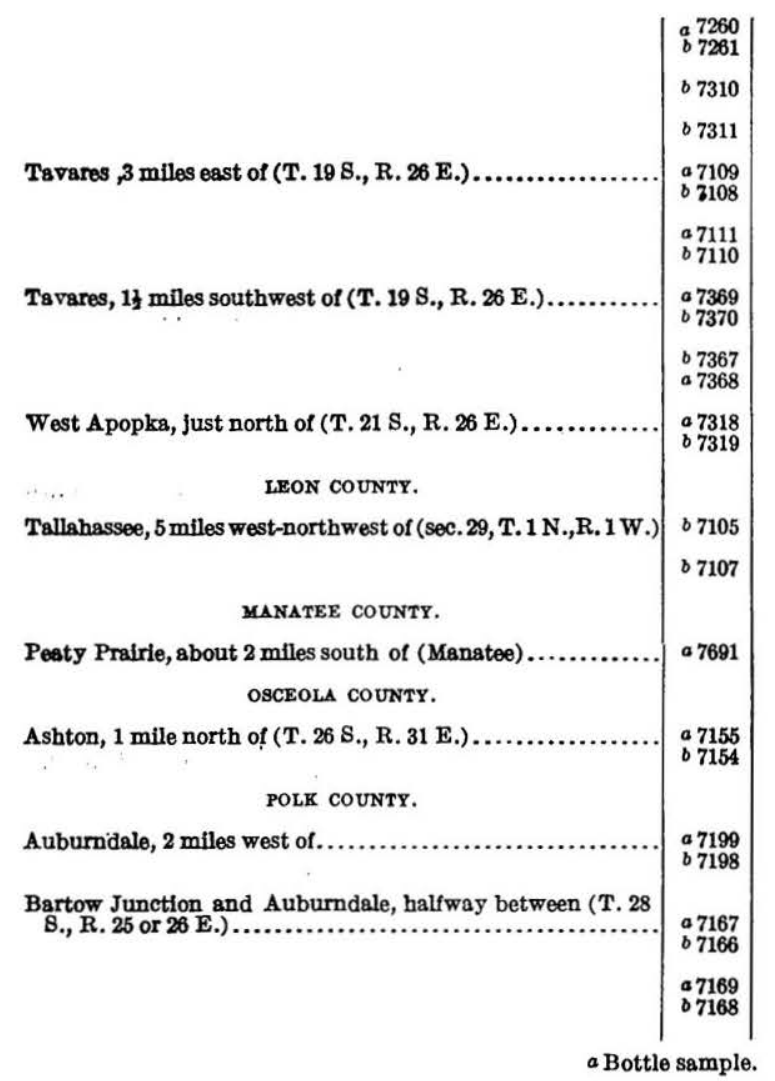

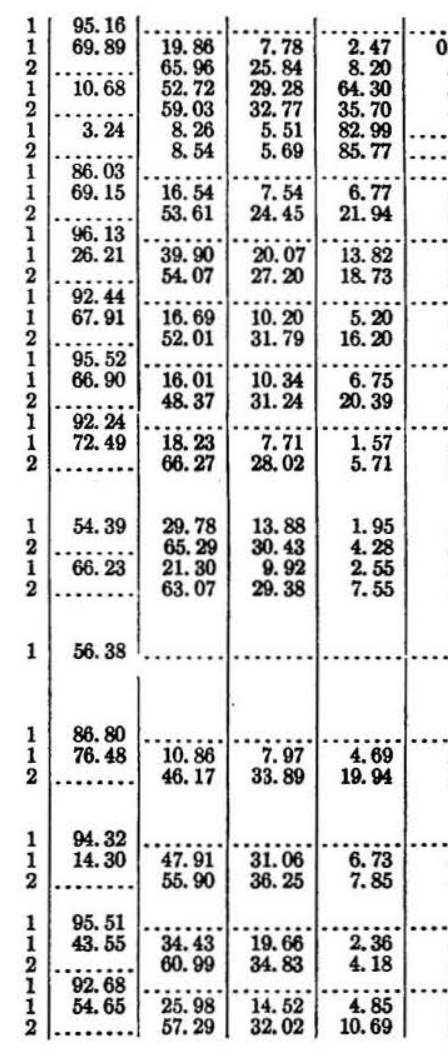

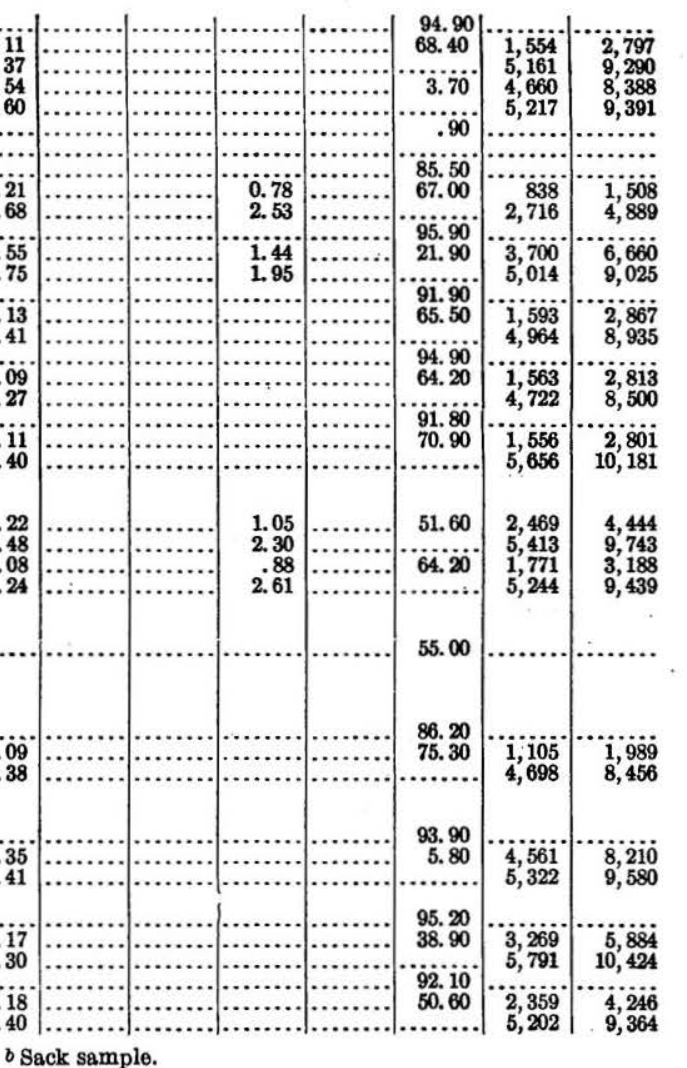

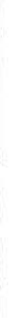


Analyses of peat and muck-Continued.

\begin{tabular}{|c|c|c|c|c|c|c|c|c|c|c|c|c|c|c|}
\hline \multirow[b]{2}{*}{ Locality. } & \multirow{2}{*}{$\begin{array}{c}\text { Labora- } \\
\text { tory } \\
\text { No. }\end{array}$} & \multirow{2}{*}{$\begin{array}{c}\text { Condi- } \\
\text { tion of } \\
\text { sam- } \\
\text { ple. }\end{array}$} & \multicolumn{4}{|c|}{ Proximate. } & \multicolumn{5}{|c|}{ Ultimate. } & \multirow{2}{*}{$\begin{array}{c}\text { Air- } \\
\text { drying } \\
\text { loss. }\end{array}$} & \multicolumn{2}{|c|}{ Calorifle value. } \\
\hline & & & $\begin{array}{l}\text { Mois- } \\
\text { ture. }\end{array}$ & $\mid \begin{array}{c}\text { Vole- } \\
\text { tlle } \\
\text { matter. }\end{array}$ & $\begin{array}{c}\text { Fyxed } \\
\text { carbon. }\end{array}$ & Ash. & $\begin{array}{c}\text { Sul- } \\
\text { phur. }\end{array}$ & $\begin{array}{c}\text { Hydro- } \\
\text { gen. }\end{array}$ & $\begin{array}{l}\text { Car- } \\
\text { bon. }\end{array}$ & $\begin{array}{c}\text { Nitro- } \\
\text { gen. }\end{array}$ & $\begin{array}{l}\text { Oxy- } \\
\text { gen. }\end{array}$ & & $\begin{array}{l}\text { Calo- } \\
\text { ries. }\end{array}$ & $\begin{array}{c}\text { British } \\
\text { thermal } \\
\text { units. }\end{array}$ \\
\hline POLK coUNTY-continued. & & & & & & & & & & & & & & \\
\hline Florence Villa, half a mile north of (T. 28 S., R. 26 E.)...... & $\begin{array}{l}a 7165 \\
b 7164\end{array}$ & $\begin{array}{l}1 \\
1\end{array}$ & $\begin{array}{l}83.82 \\
38.79\end{array}$ & 23.42 & is. $2 \mathrm{i}$ & 22.58 & 0.17 & & & & & $\begin{array}{l}82.70 \\
33.70\end{array}$ & $\dddot{2}, 163$ & $\dddot{3}, 3093$ \\
\hline Lakeland, 2 miles east of (T. 28 S., R. 24 E.).... & $\begin{array}{l}a 7201 \\
b 7200\end{array}$ & $\begin{array}{l}1 \\
1\end{array}$ & $\begin{array}{ll}90.40 \\
13.37\end{array}$ & 30.07 & $\dddot{28.9} 9$ & 2.64 & $\cdots .20$ & & & & & 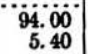 & $\dddot{5}, 0,072$ & $\cdots, 130$ \\
\hline PUTNAM COUNTY. & & 2 & & 63.57 & 33.38 & 3.05 & .39 & & & & & & 5,855 & 10,539 \\
\hline Palatka, 1 mille south of (T. 10 S., R. 27 E.)....... & $\begin{array}{l}\text { a } 7151 \\
\text { b } 7150\end{array}$ & $\begin{array}{l}1 \\
1 \\
2\end{array}$ & $\begin{array}{l}90.85 \\
80.78\end{array}$ & 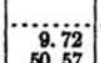 & 6. 32 & 3.18 & $7.40^{\circ}$ & & & & & $\begin{array}{l}90.30 \\
79.40\end{array}$ & 923 & 1,601 \\
\hline & $\begin{array}{l}\text { a } 7153 \\
\text { b } 7152\end{array}$ & 1 & 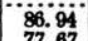 & iii & & 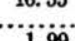 & & & & & & 86.30 & 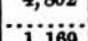 & הקוטה \\
\hline SANTA ROBA COUNTY. & & $\overline{2}$ & & 52.80 & 38.29 & 8.91 & .94 & $\ldots$ & & & & & 5,235 & 9,423 \\
\hline Miltan, near (T. 1 N., R. 28 W.).......... & $a 7021$ & 1 & 71.63 & & & & & & & & & 70.70 & & \\
\hline & b7022 & $\frac{1}{2}$ & 58. 14 & $\begin{array}{r}5.63 \\
13.45\end{array}$ & $\begin{array}{l}2.85 \\
6.81\end{array}$ & $\begin{array}{l}33.38 \\
79.74\end{array}-10-10$ & .35 & & & & $\cdots$ & 56.50 & 1.44 & $\frac{1,087}{2,597}$ \\
\hline & $\begin{array}{ll}: 7023 \\
07024\end{array}$ & 1 & $\begin{array}{l}83.50 \\
689\end{array}$ & 6. & $3.70^{\circ}$ & 2i & .60 & & & & …...... & $\begin{array}{l}82.80 \\
67.80\end{array}$ & $\cdots$ & 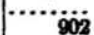 \\
\hline SUMTER COUNTY. & & & & 20.44 & 11.90 & 67.66 & 2. 12 & & & & & & 1,610 & 2,898 \\
\hline Lake Panasoffikee, south end of.. & a 7097 & 1 & 86.30 & & & & & & & & & 85.20 & & \\
\hline & $<7693$ & 1 & 93.60 & & & & & & & & ........ & $\begin{array}{r}92.30 \\
92.80\end{array}$ & & …..... \\
\hline
\end{tabular}


IIIrvors.

Analyses of moisture-free peat and muck.

[H. M. Cooper, Bureau of Mines, anslyst.]

\begin{tabular}{|c|c|c|c|c|c|c|c|c|c|}
\hline \multirow[b]{2}{*}{ County. } & \multirow{2}{*}{$\begin{array}{c}\text { Local- } \\
\text { ity } \\
\text { No. }\end{array}$} & \multirow[b]{2}{*}{$\begin{array}{c}\text { Ansly- } \\
\text { sis } \\
\text { No. }\end{array}$} & \multicolumn{3}{|c|}{ Proximste. } & \multicolumn{2}{|c|}{ Ultimate. } & \multicolumn{2}{|c|}{ Calorific value. } \\
\hline & & & $\begin{array}{c}\text { Vola- } \\
\text { tile } \\
\text { matter. }\end{array}$ & $\begin{array}{l}\text { Fixed } \\
\text { carbon. }\end{array}$ & Ash. & $\begin{array}{c}\text { Sul- } \\
\text { phur. }\end{array}$ & $\begin{array}{c}\text { Nitro- } \\
\text { gen. }\end{array}$ & $\begin{array}{l}\text { Calo- } \\
\text { ries. }\end{array}$ & \begin{tabular}{|c} 
British \\
thermsl \\
units.
\end{tabular} \\
\hline 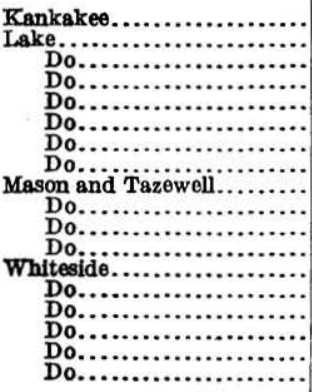 & $\begin{array}{r}2 \\
3 \\
4 \\
5 \\
6 \\
7 \\
8 \\
9 \\
13 \\
14 \\
14 \\
14 \\
15 \\
15 \\
15 \\
16 \\
16 \\
16\end{array}$ & $\begin{array}{l}317 \\
300 \\
301 \\
302 \\
303 \\
304 \\
305 \\
306 \\
313 \\
314 \\
315 \\
316 \\
307 \\
308 \\
309 \\
310 \\
311 \\
312\end{array}$ & $\begin{array}{l}41.19 \\
61.20 \\
33.27 \\
57.47 \\
67.63 \\
34.57 \\
68.15 \\
58.67 \\
63.99 \\
34.83 \\
62.70 \\
37.17 \\
65.65 \\
63.18 \\
61.51 \\
62.01 \\
50.52 \\
32.20\end{array}$ & $\begin{array}{r}29.22 \\
23.66 \\
19.33 \\
20.79 \\
23.25 \\
23.46 \\
11.24 \\
30.72 \\
9.44 \\
26.05 \\
2.96 \\
27.48 \\
6.37 \\
23.04 \\
23.86 \\
21.36 \\
6.48 \\
17.78\end{array}$ & $\begin{array}{l}29.59 \\
15.14 \\
47.40 \\
21.74 \\
91.12 \\
41.97 \\
20.61 \\
10.61 \\
26.57 \\
39.12 \\
34.34 \\
35.35 \\
27.98 \\
13.78 \\
14.63 \\
16.63 \\
43.00 \\
50.02\end{array}$ & $\begin{array}{r}2.39 \\
1.14 \\
2.26 \\
2.43 \\
.52 \\
.67 \\
1.20 \\
.66 \\
1.21 \\
.38 \\
.30 \\
.41 \\
.36 \\
.27 \\
.27 \\
.49 \\
.39 \\
.56\end{array}$ & $\begin{array}{l}3.03 \\
3.84 \\
2.18 \\
2.85 \\
2.52 \\
2.45 \\
3.18 \\
2.88 \\
2.24 \\
1.92 \\
2.97 \\
2.42 \\
2.68 \\
3.23 \\
3.30 \\
2.52 \\
2.05 \\
1.48\end{array}$ & 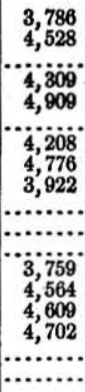 & 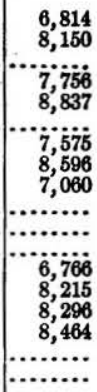 \\
\hline
\end{tabular}

INDIANA.

Analyses of moisture-free peat.

[H. M. Cooper, Bureau of Mines, analyst.]

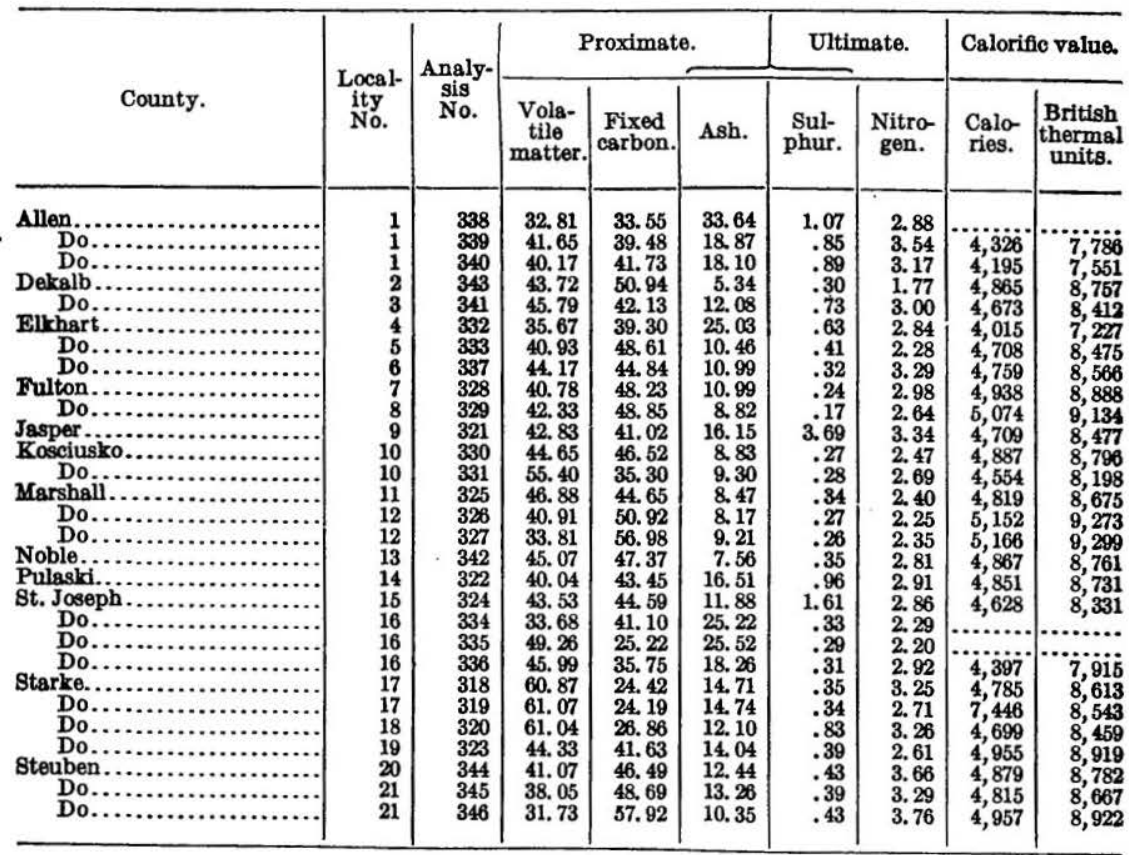


PEAT, IN THEE UNITED STATES.

\section{Analyses of air-dried peat.}

[R. E. Lyons, University of Indiana, anslyst.]

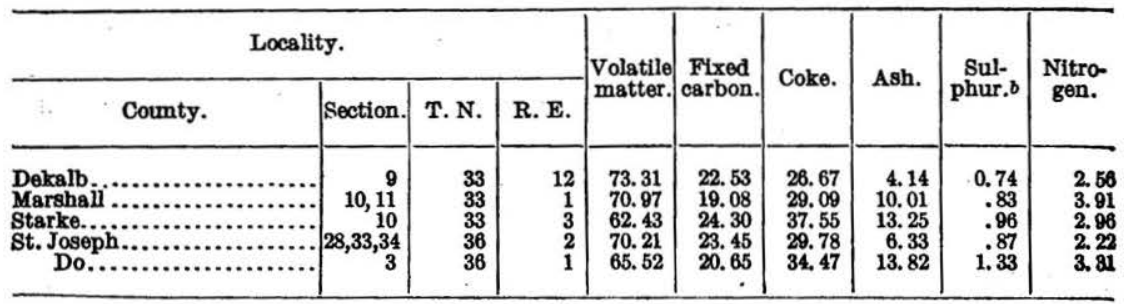

a Taylor, A. E., The pest deposits of northern Indians: Indians Dept. Geology and Nat. Res. Thirtyfirst Ann. Rept., p. 112, 1906.

o Thesamples were oven iried before the sulphur content wss determined.

Fuel value of peat oven-dried at $105^{\circ} \mathrm{C.} .^{a}$

[R. E. Lyons, University of Indians, analyst.]

\begin{tabular}{|c|c|c|c|c|c|c|}
\hline \multicolumn{4}{|l|}{ Locality. } & \multirow{2}{*}{$\begin{array}{c}\text { Sample } \\
\text { No. }\end{array}$} & \multicolumn{2}{|c|}{ Calorific value. } \\
\hline County. & Section. & T. N. & R. & & Calories. & $\begin{array}{c}\text { British } \\
\text { thermal } \\
\text { units. }\end{array}$ \\
\hline 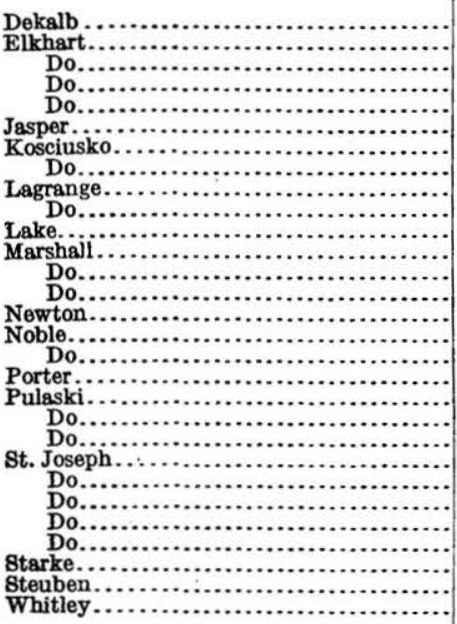 & $\begin{array}{r}9 \\
4 \\
10,11 \\
26,27 \\
18 \\
12,13,14 \\
11,12,13 \\
32,33 \\
2,11,12 \\
4,9 \\
34,35,36 \\
10,11 \\
1 \\
10 \\
32,33 \\
28,29 \\
18 \\
1,2,3 \\
7,9 \\
7,8,9 \\
3,9,10,11 \\
28,33,34 \\
3 \\
11,12 \\
16 \\
20 \\
10 \\
34 \\
30\end{array}$ & $\begin{array}{l}33 \\
36 \\
36 \\
35 \\
38 \\
30 \\
31 \\
33 \\
36 \\
37 \\
35 \\
33 \\
34 \\
34 \\
31 \\
33 \\
33 \\
37 \\
31 \\
31 \\
31 \\
36 \\
36 \\
37 \\
37 \\
37 \\
32 \\
37 \\
31\end{array}$ & $\begin{array}{rl}12 & \mathrm{E} . \\
5 & \mathrm{E} . \\
6 & \mathrm{E} . \\
5 & \mathrm{E} . \\
6 & \mathrm{E} . \\
6 & \mathrm{~W} . \\
6 & \mathrm{E} . \\
6 & \mathrm{E} . \\
8 & \mathrm{E} . \\
9 & \mathrm{E} . \\
9 & \mathrm{~W} . \\
1 & \mathrm{E} . \\
2 & \mathrm{E} . \\
1 & \mathrm{E} . \\
8 & \mathrm{~W} . \\
9 & \mathrm{E} . \\
11 & \mathrm{E} . \\
5 & \mathrm{~W} . \\
1 & \mathrm{~W} . \\
3 & \mathrm{~W} . \\
4 & \mathrm{~W} . \\
2 & \mathrm{E} . \\
1 & \mathrm{E} . \\
1 & \mathrm{E} . \\
2 & \mathrm{E} . \\
2 & \mathrm{E} . \\
3 & \mathrm{E} . \\
12 & \mathrm{E} . \\
10 & \mathrm{E} .\end{array}$ & $\begin{array}{r}1 \\
10 \\
11 \\
12 \\
13 \\
27 \\
8 \\
9 \\
3 \\
4 \\
29 \\
19 \\
20 \\
21 \\
28 \\
5 \\
6 \\
26 \\
23 \\
24 \\
14 \\
15 \\
16 \\
17 \\
18 \\
22 \\
2 \\
7\end{array}$ & $\begin{array}{l}5,685 \\
4,799 \\
4,006 \\
4,229 \\
5,349 \\
4,596 \\
5,398 \\
3,405 \\
4,730 \\
4,958 \\
4,851 \\
5,526 \\
4,721 \\
5,815 \\
5,019 \\
5,742 \\
5,121 \\
3,131 \\
5,430 \\
5,036 \\
4,707 \\
5,467 \\
5,013 \\
4,724 \\
4,584 \\
4,718 \\
5,503 \\
5,234 \\
2,523\end{array}$ & $\begin{array}{r}10,233 \\
8,638 \\
7,211 \\
7,613 \\
9,629 \\
8,273 \\
9,716 \\
6,126 \\
8,511 \\
8,924 \\
8,731 \\
9,946 \\
8,498 \\
10,466 \\
9,036 \\
10,336 \\
9,217 \\
5,635 \\
9,775 \\
9,065 \\
9,065 \\
9,940 \\
9,024 \\
8,504 \\
8,236 \\
8,491 \\
9,906 \\
9,423 \\
4,542\end{array}$ \\
\hline
\end{tabular}

a Taylor, A. E., op. cit., p. 111. 
PROPERTIES OF PEAT.

IOWA.

Analyses of air-dried peat. ${ }^{\circ}$

\begin{tabular}{|c|c|c|c|c|c|c|c|}
\hline \multicolumn{2}{|c|}{ Locslity. } & \multirow[b]{2}{*}{$\begin{array}{l}\text { Labora- } \\
\text { tory No. }\end{array}$} & \multicolumn{4}{|c|}{ Proximate. } & \multirow{2}{*}{$\begin{array}{c}\text { Calorific } \\
\text { value } \\
\text { (British } \\
\text { thermsl } \\
\text { units). }\end{array}$} \\
\hline County. & Township. & & Moisture. & $\begin{array}{l}\text { Volstile } \\
\text { and com- } \\
\text { bustible } \\
\text { matter. }\end{array}$ & $\begin{array}{c}\text { Fixed } \\
\text { carbon. }\end{array}$ & Ash. & \\
\hline 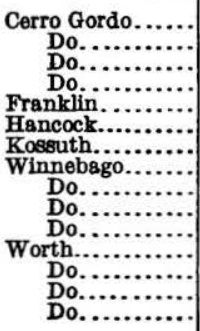 & 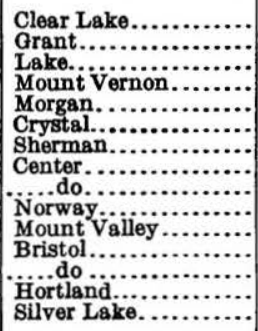 & $\begin{array}{l}594 \\
595 \\
598 \\
596 \\
354 \\
647 \\
636 \\
514 \\
499 \\
597 \\
563 \\
489 \\
490 \\
533 \\
492\end{array}$ & $\begin{array}{r}7.30 \\
7.97 \\
11.81 \\
7.99 \\
8.66 \\
7.55 \\
10.51 \\
10.07 \\
10.85 \\
11.91 \\
5.89 \\
19.74 \\
19.61 \\
11.27 \\
18.26\end{array}$ & $\begin{array}{l}50.71 \\
52.94 \\
45.03 \\
52.61 \\
52.47 \\
54.57 \\
51.61 \\
55.10 \\
50.71 \\
50.23 \\
63.59 \\
49.60 \\
48.07 \\
51.18 \\
49.57\end{array}$ & $\begin{array}{l}17.10 \\
16.60 \\
23.51 \\
15.47 \\
15.38 \\
15.67 \\
13.80 \\
14.65 \\
13.29 \\
17.02 \\
16.39 \\
12.18 \\
13.49 \\
13.32 \\
13.03\end{array}$ & $\begin{array}{l}24.89 \\
22.49 \\
19.66 \\
23.93 \\
23.49 \\
22.21 \\
24.09 \\
20.18 \\
25.15 \\
20.84 \\
14.13 \\
18.48 \\
18.83 \\
24.23 \\
19.14\end{array}$ & $\begin{array}{l}6,452 \\
7,129 \\
6,125 \\
6,904 \\
7,699 \\
7,278 \\
6,811 \\
7,418 \\
7,169 \\
6,966 \\
8,431 \\
6,624 \\
6,764 \\
6,951 \\
6,889\end{array}$ \\
\hline
\end{tabular}

a Beyer, S. W., Peat deposits in Iowa: Iowa Geol. Survey, vol. 19, pp. 725-730, 1908. 
Analyses of peat and muck.a

[F. M. Stanton, U. 8. Geological Survey, analyst.]

\begin{tabular}{|c|c|c|c|c|c|c|c|c|c|c|c|c|c|c|}
\hline \multirow{2}{*}{ Locality. } & \multirow{2}{*}{$\begin{array}{c}\text { Labora } \\
\text { tory. } \\
\text { No. }\end{array}$} & \multirow{2}{*}{$\begin{array}{l}\text { Condi- } \\
\text { tion of } \\
\text { sam. } \\
\text { ple. }\end{array}$} & \multicolumn{3}{|c|}{ Proximate. } & \multirow[b]{2}{*}{ Ash. } & \multirow[b]{2}{*}{$\begin{array}{c}\text { Sul- } \\
\text { phur. }\end{array}$} & \multicolumn{2}{|c|}{ Ultimate. } & & \multirow{2}{*}{$\begin{array}{l}\text { Air- } \\
\text { drying } \\
\text { loss. }\end{array}$} & \multicolumn{2}{|c|}{ Calorifle value. } \\
\hline & & & $\begin{array}{l}\text { Mois- } \\
\text { ture. }\end{array}$ & $\begin{array}{c}\text { Vola- } \\
\text { tile } \\
\text { matter. }\end{array}$ & $\begin{array}{l}\text { Fixed } \\
\text { carbon. }\end{array}$ & & & $\begin{array}{c}\text { Hydro- } \\
\text { gen. }\end{array}$ & $\begin{array}{l}\text { Car- } \\
\text { bon. }\end{array}$ & $\begin{array}{c}\text { Nitro- } \\
\text { gen. }\end{array}$ & $\begin{array}{l}\text { Oxy- } \\
\text { gen. }\end{array}$ & & Calories. & $\begin{array}{l}\text { British } \\
\text { thermal } \\
\text { units. }\end{array}$ \\
\hline$\triangle N D R O S C O G G N$ COUNTY. & & & & & & & & & & & & & & \\
\hline Greene,near............................... & 5895 & 1 & 86. 52 & 7.55 & 4.22 & 1.71 & 0.08 & & & 0.24 & & 85.00 & 655 & 1,179 \\
\hline Lewiston, 2 miles east of (Garcelon Bog)... & 5863 & 1 & $\dddot{82.7 i}$ & $\begin{aligned} 56.01 \\
\ldots \ldots \ldots . .\end{aligned}$ & $\begin{array}{r}31.30 \\
\cdots \cdots \cdots\end{array}$ & $\begin{array}{r}12.69 \\
1.16\end{array}$ & $\begin{array}{l}.59 \\
.02\end{array}$ & $\cdots \cdot$ & -........... & $\begin{array}{l}1.78 \\
.20\end{array}$ & & $\dddot{80.80}$ & $\begin{array}{r}4,859 \\
892\end{array}$ & $\begin{array}{l}8,746 \\
1,606\end{array}$ \\
\hline Lewiston, 2 miles east of.. & 5897 & 1 & 36.98 & 7.0 .04 & 4.40 & $\begin{array}{l}6.71 \\
.78\end{array}$ & .02 & $\cdots . .$. & …........ & $\begin{array}{l}1.16 \\
.13\end{array}$ & $\cdots$ & $\ddot{84} \ddot{20}$ & $\begin{array}{r}5,159 \\
682\end{array}$ & $\begin{array}{l}9,286 \\
1,228\end{array}$ \\
\hline & 5894 & 1 & 85.91 & 8.43 & 4. 37 & 1. 29 & .07 & $\mid \cdots . . . . . .6$ & $\cdots \cdots . . . \cdot$ & 1.03 & & $\dddot{84} .60$ & 5, 238 & $\begin{array}{l}9,428 \\
1,287\end{array}$ \\
\hline Lowiston, 2 miles east of (machine peat) $\ldots \ldots \ldots \ldots \ldots \ldots \ldots$ & 5865 & $\begin{array}{l}2 \\
1\end{array}$ & 65.39 & $\begin{aligned} 59.83 \\
\ldots \ldots \ldots\end{aligned}$ & $\begin{array}{c}31.01 \\
\ldots \ldots \ldots\end{array}$ & $\begin{array}{l}9.16 \\
1.55\end{array}$ & .50 & ............ & …........ & $\begin{array}{r}1.63 \\
.46\end{array}$ & $\cdots$ & $\dddot{60.40}$ & $\begin{array}{l}5,057 \\
1,127\end{array}$ & 9,020 \\
\hline Lewiston, 4 miles east of (bog south of No Name Pond)....... & 5864 & $\stackrel{2}{1}$ & 87.55 & $\cdots \cdots$ & ........ & $\begin{array}{r}4.48 \\
.67\end{array}$ & .49 & $\cdots$ & $\cdots \cdots$ & $\begin{array}{r}1.33 \\
.14\end{array}$ & $\cdots$ & $\ddot{86.10}$ & $\begin{array}{r}3,256 \\
610\end{array}$ & $\begin{array}{l}5,861 \\
1,098\end{array}$ \\
\hline Lewiston, 4 miles east of . ................. & 5866 & .2 & $\dddot{88 . ~} \dddot{67}$ & 3.83 & 2.95 & $\begin{array}{r}5.38 \\
2.55\end{array}$ & .02 & $\mid \cdots . . . . . .$. & -.......... & $\begin{array}{l}1.12 \\
.13\end{array}$ & $\cdots \cdots .$. & $\because 77,60$ & $\begin{array}{r}4,900 \\
460\end{array}$ & 8,8200 \\
\hline & 5896 & 常 & $\dddot{85.99}$ & 81.46 & $\begin{array}{r}20.03 \\
3.99\end{array}$ & $\begin{array}{r}22.51 \\
2.51\end{array}$ & .05 & $|\cdots \cdots \cdots \cdot|$ & (n.......... & $\begin{array}{r}1.15 \\
.20\end{array}$ & (n......... & 3480 & $\begin{array}{r}4,060 \\
634\end{array}$ & $\begin{array}{l}7,308 \\
1,141\end{array}$ \\
\hline AROOSTOOK COUNTY. & & & n........ & & & & & ... & & & & & & 7,864 \\
\hline Crystal, 2 miles southwest of ............. & 5912 & 1 & 85.02 & ......... & …...... & 1.41 & .04 & & & .23 & & 83.61 & 763 & 1,373 \\
\hline Houlton, 1 mile southwest of........... & 5913 & 1 & $\dddot{87.47}$ & ........... & …........ & .75 & .02 & $\mid \cdots . . . \cdots$ & $\cdots \ldots . . . \cdot$ & 16 & ....... & $86.30^{\circ}$ & 678 & $\begin{array}{l}9,167 \\
1,220\end{array}$ \\
\hline Sherman, near...... & 5914 & 1 & 86. 18 & 8.27 & 3.98 & $\begin{array}{l}5.99 \\
1.57\end{array}$ & .10 & 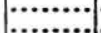 & …....... & 1.28 & …...... & $\ddot{82} 80^{\circ}$ & 5, 411 & $\begin{array}{l}9,740 \\
1,294\end{array}$ \\
\hline CUMBERLAND COUNTY. & & & $\cdots \cdot$ & 59.84 & 28.80 & 11.36 & .72 & ......... & $\cdots \cdots$ & 2.03 & & & 5,203 & 9,365 \\
\hline Cape Elizabeth, near... & 5861 & 1 & 85.87 & 8.12 & 4.25 & 1.76 & .14 & .......... & ........... & .26 & 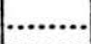 & 84.00 & 701 & 1,262 \\
\hline & 5995 & 1 & 84. 44 & 8.93 & 4.95 & 1.68 & .17 & .......... & ....... & & & $\dddot{82} 00$ & & \\
\hline Cumberland Mills, 5 by miles north of ... & 5844 & 1 & 91.00 & $\begin{array}{r}5.39 \\
5.41\end{array}$ & $\begin{array}{l}31.81 \\
2.60\end{array}$ & $\begin{array}{r}10.80 \\
.99\end{array}$ & .09 & $\left|\begin{array}{ll}n \\
\cdots \cdots \cdots\end{array}\right|$ & …........ & or & (............ & 89.20 & 419 & $77_{4}$ \\
\hline & 5836 & $\begin{array}{l}2 \\
1 \\
2\end{array}$ & 89.59 & $\begin{array}{r}6.42 \\
61.67\end{array}$ & $\begin{array}{r}3.27 \\
31.41\end{array}$ & $\begin{array}{r}.72 \\
6.92\end{array}$ & $\begin{array}{l}.11 \\
.01 \\
.10\end{array}$ & $\ldots . .$. & ….......... & .78 & (…....... & 88.30 & $\begin{array}{r}4,656 \\
521 \\
5,005\end{array}$ & $\begin{array}{l}8,381 \\
938 \\
9,000\end{array}$ \\
\hline
\end{tabular}




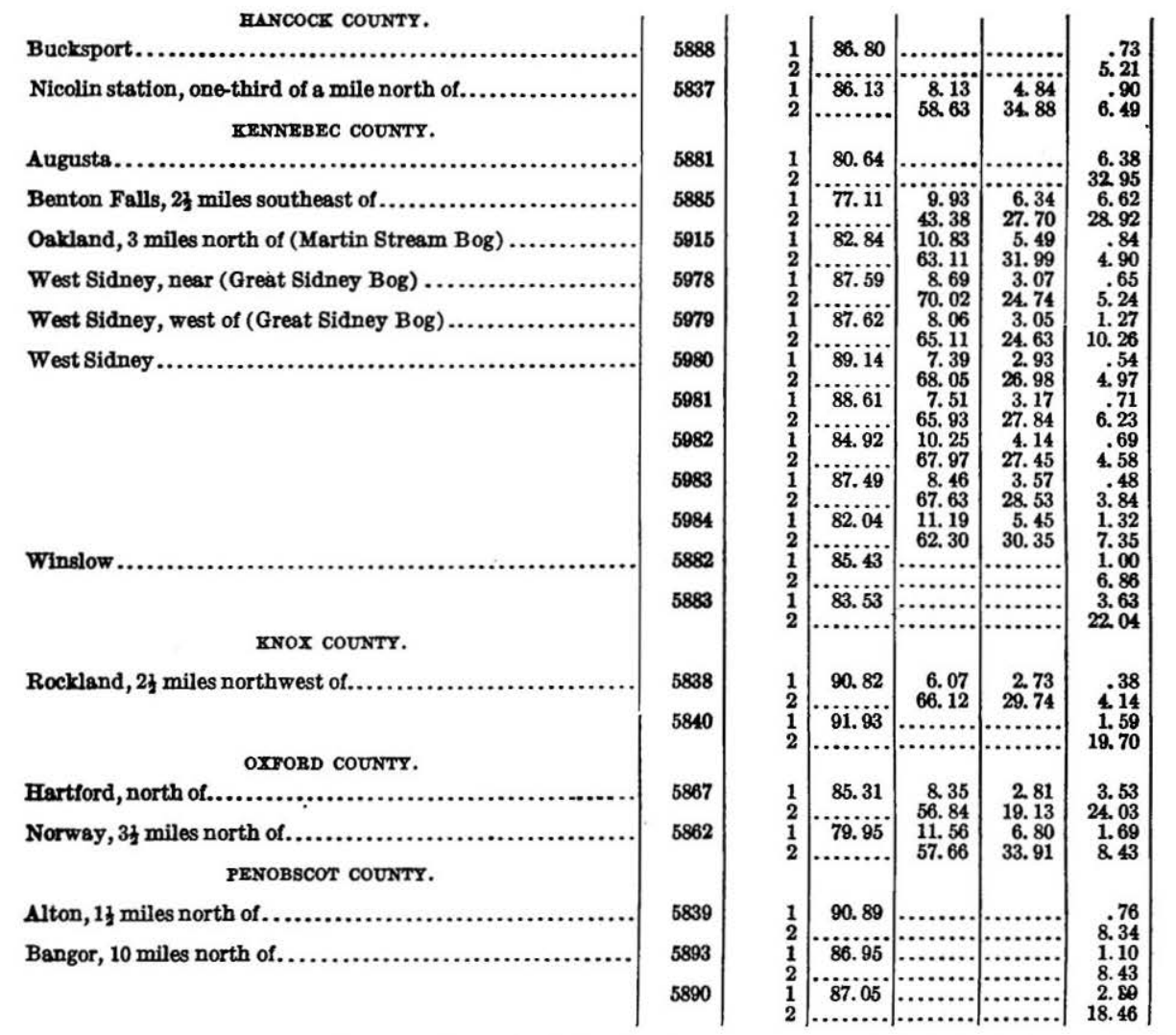

a Davis, C. A., The uses of peat for fuel and other purposes: Bur. Mines Bull. 16, pp. 192-196, 1911.

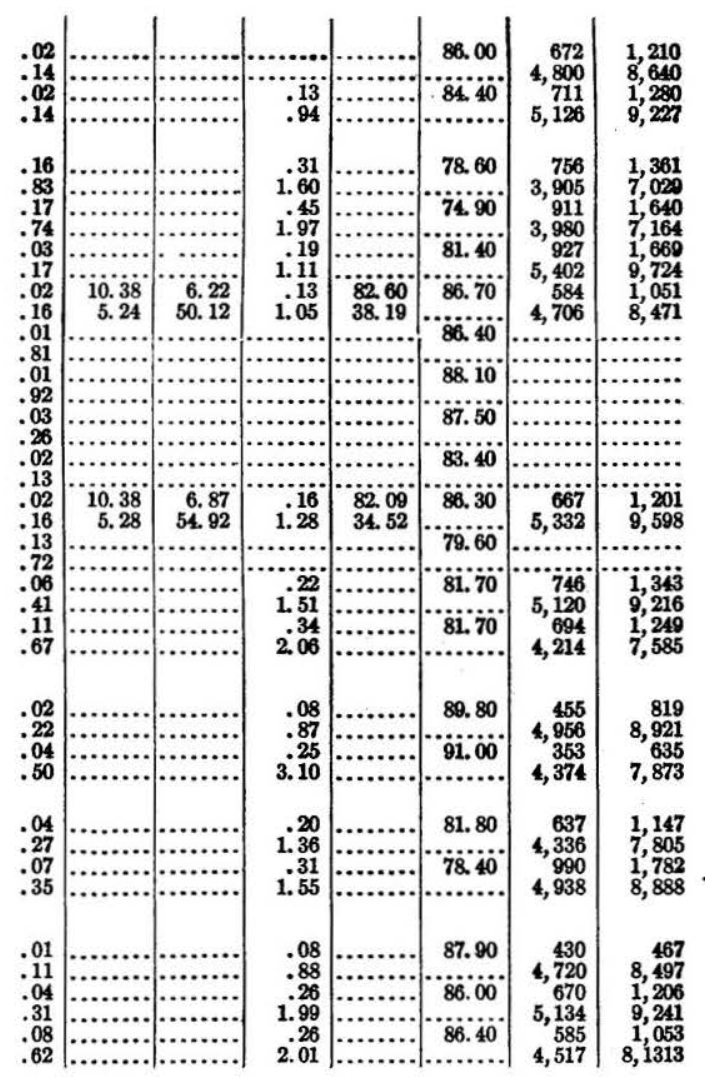

b 1, Native peat. 2, Moisture-free peat. 


\begin{tabular}{|c|c|c|c|c|c|c|c|c|c|c|c|c|c|c|}
\hline \multirow[b]{2}{*}{ Locality. } & \multirow{2}{*}{$\begin{array}{c}\text { Labora- } \\
\text { tory. } \\
\text { No. }\end{array}$} & \multirow{2}{*}{$\begin{array}{c}\text { Condi- } \\
\text { tion of } \\
\text { sam- } \\
\text { ple. }\end{array}$} & \multicolumn{4}{|c|}{ Proximate. } & \multicolumn{5}{|c|}{ Ultimate. } & \multirow{2}{*}{$\begin{array}{l}\text { Air- } \\
\text { drying } \\
\text { losis. }\end{array}$} & \multicolumn{2}{|c|}{ Calorific value. } \\
\hline & & & $\begin{array}{l}\text { Mois- } \\
\text { ture. }\end{array}$ & $\left|\begin{array}{c}\text { Volar } \\
\text { tile } \\
\text { matter. }\end{array}\right|$ & Fixed & Ash. & $\begin{array}{c}\text { Sul- } \\
\text { phur. }\end{array}$ & $\begin{array}{c}\text { Hydro- } \\
\text { gen. }\end{array}$ & $\begin{array}{l}\text { Car- } \\
\text { bon. }\end{array}$ & $\begin{array}{c}\text { Nitro- } \\
\text { gen. }\end{array}$ & $\begin{array}{l}\text { Oxy- } \\
\text { gen. }\end{array}$ & & $\begin{array}{l}\text { Calo- } \\
\text { ries. }\end{array}$ & \begin{tabular}{|l} 
British \\
thermal \\
units.
\end{tabular} \\
\hline 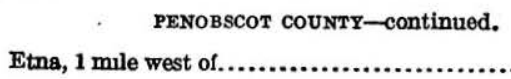 & 5891 & & 84.68 & & & & & & & & & & & \\
\hline Hermon Center, west of.......................... & 5902 & $\begin{array}{l}2 \\
1\end{array}$ & 86. & :...... & & $\begin{array}{r}8.68 \\
1.50\end{array}$ & $\begin{array}{l}.65 \\
.07\end{array}$ & & & $\begin{array}{r}2.28 \\
.29\end{array}$ & & $\because 850$ & $\begin{array}{l}5,085 \\
-678\end{array}$ & $\begin{array}{l}9,153 \\
1,220\end{array}$ \\
\hline & 5892 & 1 & 88.23 & .............. & …....... & 1.15 & .06 .06 & 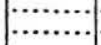 & ....... & $\begin{array}{l}2.14 \\
.25\end{array}$ & …... & 87.00 & 5,015 & $\begin{array}{l}9,027 \\
1,057\end{array}$ \\
\hline Hermon Pond, southwest of .................. & 5916 & 1 & 89.01 & 6.3 & 3.43 & $\begin{array}{r}9.77 \\
.63\end{array}$ & .51 & & $\cdots .$. & $\begin{array}{r}2.12 \\
.23\end{array}$ & & $\because 36.80$ & 4,987 & $\begin{array}{l}8,977 \\
1,058\end{array}$ \\
\hline Newport, three-fourths of a mile east of... & 5889 & $\begin{array}{l}2 \\
1\end{array}$ & 80.00 & $\mid \begin{array}{r}63.08 \\
\ldots . . . . .6\end{array}$ & 31.21 & $\begin{array}{l}5.73 \\
1.09\end{array}$ & $\begin{array}{l}.36 \\
.02\end{array}$ & . & ....... & $\begin{array}{l}2.09 \\
.17\end{array}$ & & 84.80 & $\begin{array}{r}5,350 \\
736\end{array}$ & $\begin{array}{l}9,630 \\
1,325\end{array}$ \\
\hline Spragues, near............................. & 5841 & 1 & 88.34 & …...... & ….... & .84 & .02 & & ........ & 1.18 & $\ldots$. & 85.00 & $\begin{array}{l}5,108 \\
597\end{array}$ & $\begin{array}{l}9,194 \\
1,075\end{array}$ \\
\hline SOMERSET COUNTY. & & & & & & & & & & & & & 5,120 & 9,216 \\
\hline Oakland, 4 miles north of............... & 5884 & 1 & 83.64 & & & .80 & .03 & & 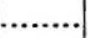 & .21 & $\ldots$ & 82.20 & 872 & 1,570 \\
\hline & 5880 & 1 & 83.66 & ........... & …....... & $\begin{array}{l}4.08 \\
4.09\end{array}$ & .11 & & ........ & $\begin{array}{l}1.28 \\
.26\end{array}$ & ․ & $82.30^{\circ}$ & 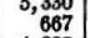 & $\begin{array}{l}9,594 \\
1,201\end{array}$ \\
\hline Pittsfield, 1 mile southeast of ............ & 5994 & & 90.08 & 6.41 & 2.10 & .61 & .01 & 10.58 & 4.41 & $\begin{array}{r}1.09 \\
.09\end{array}$ & 84. & 90.20 & $\begin{array}{lll}4,462 \\
412\end{array}$ & $\begin{array}{l}7,348 \\
742\end{array}$ \\
\hline & 5886 & 1 & $89.80^{\circ}$ & & & .64 & .02 & & & .10 & & 89.10 & , 4018 & $\begin{array}{lll}8,132 \\
9225\end{array}$ \\
\hline 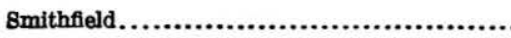 & 5887 & ${ }_{1}^{2}$ & 86.06 & & ........ & 6. 62 & .04 & & & .99 & & 35.20 & $\begin{array}{c}5,064 \\
813\end{array}$ & 9,115 \\
\hline WASHINGTON COUNTY. & & 2 & & & & 4.45 & .29 & & $\cdots$ & 1.36 & & & 5,832 & 10,498 \\
\hline$\Delta$ yers Junction, half a mile east of........ & 5933 & 1 & 85.22 & 8.86 & 4.72 & 1.20 & .07 & & & & & 83.80 & & 1,444 \\
\hline Ayers Junction, 13 miles southwest of .......... & 5904 & 1 & $\because 87.61$ & & 31.93 & 8.12 & .02 & & & iii & & $86.40^{\circ}$ & $\begin{array}{l}5.433 \\
657\end{array}$ & 9,779 \\
\hline & 5903 & & $88.2 \mathrm{i}^{\circ}$ & & …..... & $\begin{array}{c}0.60 \\
.66\end{array}$ & .02 & $\ldots$ & ....... & .10 & . & 87.10 & 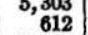 & $\begin{array}{l}y, 500 \\
1,102\end{array}$ \\
\hline 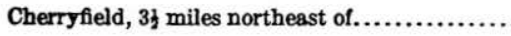 & 5909 & 1 & $\dddot{\theta} 1 \dddot{2} \dddot{2}$ & & (n...... & $.84^{\circ}$ & .01 & & $\cdots . .$. & .85 & $\cdots$ & 90.50 & $\begin{array}{l}5,191 \\
598\end{array}$ & 9,346 \\
\hline East Machias, 7 miles north of. & 5906 & $\underline{1}$ & "0i. & & …....... & .45 & .01 & & ........ & .80 & …....... & "थi. & 年, 403 & 725 \\
\hline
\end{tabular}


Falls Point, 1 mile northwest of..

Forest, northwest of

Jonesboro.

Shoppers Point, $1 \frac{1}{2}$ miles northeast of.

Southern Inlet, east of.

South Lubec, three-fourths of a mile southeast of.

Vanceboro, 2 miles west of.

Whitneyville, 2 miles west of.

YORK COUNTY.

Kittery (Cutts Island, salt marsh)

Do...............

Do.

Kittery Point (salt marsh). Do......................

Rosemary Junction (salt marsh)

Rosemary Junction (Rosemary Swamp)

$$
\text { Do. }
$$

Do...
Do...
5976

\section{7}

5908

5911

5907

5905

5975

5842

5843

5910

$a 6552$
$b 6553$

a 6556

$a$
$b$
$b$ 6555

a 6565

b 6566

a 6567
b 6568

\begin{tabular}{|c|c|c|c|c|c|c|c|c|c|c|c|c|}
\hline & 89.76 & 7.10 & 2.94 & 20 & 2 & & & & & 89.00 & & 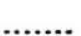 \\
\hline & 91.51 & $\begin{array}{r}69.34 \\
5.50\end{array}$ & $\begin{array}{r}28.71 \\
2.63\end{array}$ & $\begin{array}{r}1.95 \\
.36\end{array}$ & $\begin{array}{l}.20 \\
.02\end{array}$ & & & & & 90.90 & & ..... \\
\hline & 9004 & 64.78 & 30.98 & & .24 & & & & & & & \\
\hline & 90.04 & & & $\begin{array}{r}.57 \\
5.72\end{array}$ & .02 & & & .12 & & 89.10 & $\begin{array}{r}520 \\
5\end{array}$ & 936 \\
\hline & 90.92 & $\cdots \cdot$ & $\cdots . .$. & .48 & .02 & & & .07 & & 90.00 & $\begin{array}{r}0,241 \\
448\end{array}$ & 9,398 \\
\hline & 92.87 & ....... & ;............. & $\begin{array}{r}5.29 \\
.55\end{array}$ & .02 & & & $\begin{array}{l}.77 \\
.05\end{array}$ & & 92.30 & $\begin{array}{l}4,934 \\
331\end{array}$ & $\begin{array}{l}881 \\
596\end{array}$ \\
\hline & 89.62 & $\ldots \ldots$ & ... & $\begin{array}{r}7.71 \\
.43\end{array}$ & $\begin{array}{l}.28 \\
.02\end{array}$ & & & .70 & & 08 & 4,642 & 8,3 \\
\hline & 89.02 & & & 4.14 & .19 & & & .08 & & 88.70 & $\begin{array}{r}519 \\
5,000\end{array}$ & 934 \\
\hline & 88.41 & 7.72 & 3.43 & .44 & .03 & 10.44 & 6.06 & .09 & 82.94 & $\dddot{87.50}$ & 574 & 33 \\
\hline & 91.55 & 66.61 & & $\begin{array}{r}3.80 \\
.68\end{array}$ & .28 & & & $\begin{array}{r}.78 \\
.06\end{array}$ & & 8980 & $\begin{array}{l}4,953 \\
400\end{array}$ & 5 \\
\hline & & & & 8.05 & .12 & & & .71 & & & 4,734 & \\
\hline & 89.65 & $\begin{array}{r}6.33 \\
61.16\end{array}$ & $\begin{array}{r}3.15 \\
30.43\end{array}$ & $\begin{array}{r}.87 \\
8.41\end{array}$ & .02 & ….......... & $\cdots \cdots$ & .13 & $\cdots \cdots$ & 87.60 & 536 & 965 \\
\hline & 90.28 & ........... & & $\begin{array}{r}0.71 \\
.52 \\
\end{array}$ & .02 & & & .07 & & 89.20 & $\begin{array}{r}0,178 \\
478\end{array}$ & $\begin{array}{l}{ }^{3}, 322 \\
860\end{array}$ \\
\hline & & $\cdots$ & $\ldots . . . . . .$. & 5.35 & & & & .72 & & $\ldots \ldots . . .$. & 4,918 & 8,852 \\
\hline & & & & & & & & & & 88.30 & & \\
\hline & & 33.18 & 19.89 & 38.76 & 1.69 & & & & & 3.60 & 2,576 & 74637 \\
\hline & 80.13 & & & & & & & & & 79000 & & \\
\hline & 9.06 & 27.17 & 11. 20 & 52.57 & 1.99 & & & $\cdots \cdots$ & & 5.10 & 1,836 & 3,305 \\
\hline & 87.51 & & & & 2.19 & $\cdots$ & & & & & & \\
\hline & 13.50 & 49.06 & 25.40 & 12.04 & $\because 794$ & & & 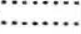 & & $\begin{array}{r}5.10 \\
\end{array}$ & 4,066 & 7,319 \\
\hline & & & & & 2.24 & & & & & 85. & & \\
\hline & 14.26 & 41.20 & 22.00 & 22.54 & 1.32 & .... & & $\cdots$ & $\ldots$ & 7.30 & $3,34 i$ & $\dddot{6}, 374$ \\
\hline & $88.1 i$ & & & & & & & & & 87. & 4,130 & 7,4 \\
\hline & & & & & 1. & & & & & 5.00 & 2,632 & 4,7 \\
\hline & & & & 6.97 & 1.46 & & & & & & 2,955 & 319 \\
\hline
\end{tabular}

b Sack sample. 
MASSAOEUSETTS.

Analyses of moisture-free peat and muck.

[H. M. Cooper, Bureau of Mines, analyst.]

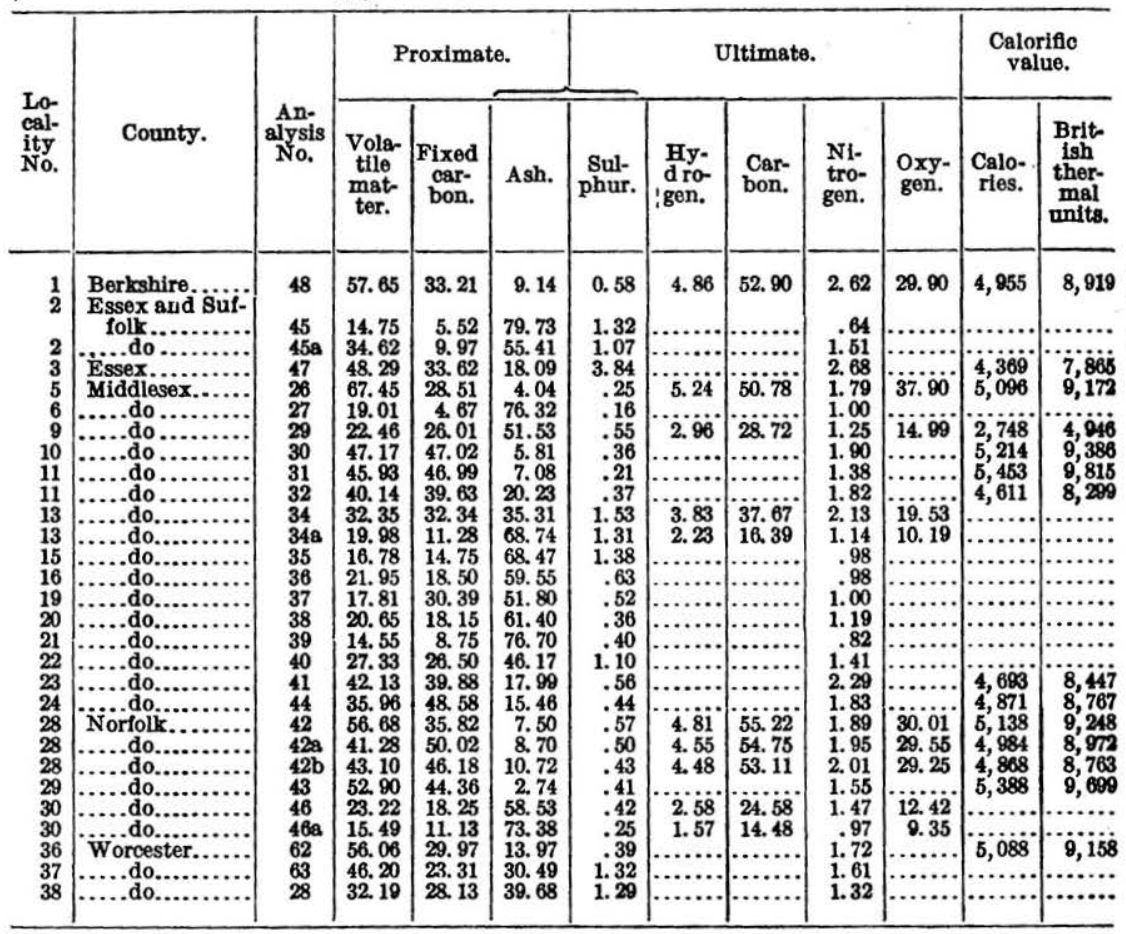

Analyses of peat. ${ }^{a}$

[F. M. Stanton, U. S. Geological Survey, analyst.

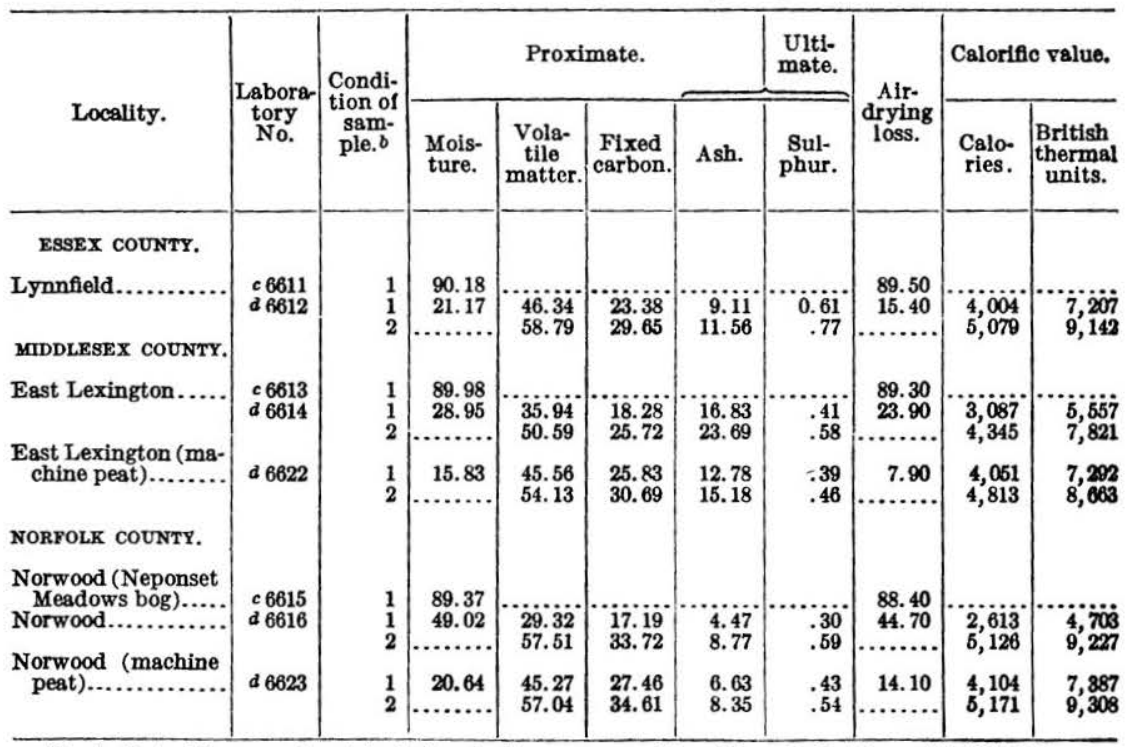

a Davis, C. A., The uses of peat for fuel and other purposes: Bur. Mines Bull. 16, pp. 196-197, 1911. b1, Native peat. 2, Moisture-free peat. c Bottle sample. d Back sample. 
MIOHIGAN.

Analyses of moisture-free peat and muck.

[H. M. Cooper, Bureau of Mines, analyst.]

\begin{tabular}{|c|c|c|c|c|c|c|c|c|c|}
\hline \multirow{2}{*}{$\begin{array}{c}\text { Local- } \\
\text { ity } \\
\text { No. }\end{array}$} & \multirow[b]{2}{*}{ County. } & \multirow{2}{*}{$\begin{array}{c}\text { Ansly- } \\
\text { sis } \\
\text { No. }\end{array}$} & \multicolumn{3}{|c|}{ Proximate. } & \multicolumn{2}{|c|}{ Ultimate. } & \multicolumn{2}{|c|}{ Calorific value. } \\
\hline & & & $\begin{array}{c}\text { Vola- } \\
\text { tile } \\
\text { matter. }\end{array}$ & $\begin{array}{l}\text { Fixed } \\
\text { carbon. }\end{array}$ & Ash. & $\begin{array}{l}\text { Sul- } \\
\text { phur. }\end{array}$ & $\begin{array}{l}\text { Nitro- } \\
\text { gen. }\end{array}$ & $\begin{array}{l}\text { Calo- } \\
\text { ries. }\end{array}$ & $\begin{array}{c}\text { British } \\
\text { thermal } \\
\text { units. }\end{array}$ \\
\hline $\begin{array}{r}1 \\
2 \\
2 \\
3 \\
3 \\
3 \\
4 \\
5 \\
6 \\
7 \\
8 \\
9 \\
10 \\
10 \\
10 \\
11 \\
12 \\
13 \\
14 \\
15 \\
15 \\
15 \\
15 \\
16 \\
17 \\
18 \\
18 \\
18 \\
19 \\
19 \\
19 \\
20 \\
20 \\
20\end{array}$ & 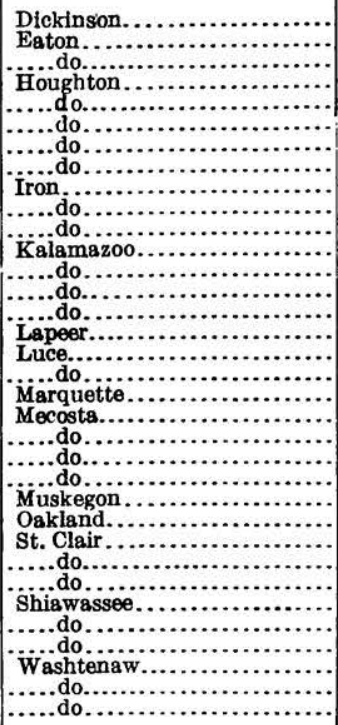 & $\begin{array}{l}383 \\
351 \\
352 \\
372 \\
373 \\
375 \\
378 \\
379 \\
380 \\
381 \\
382 \\
347 \\
348 \\
349 \\
350 \\
357 \\
369 \\
370 \\
371 \\
365 \\
366 \\
367 \\
368 \\
364 \\
356 \\
358 \\
359 \\
360 \\
361 \\
362 \\
363 \\
353 \\
354 \\
355\end{array}$ & $\begin{array}{l}54.39 \\
36.66 \\
43.82 \\
39.68 \\
39.28 \\
45.18 \\
55.83 \\
55.34 \\
49.47 \\
51.91 \\
41.08 \\
35.92 \\
34.51 \\
35.91 \\
37.16 \\
46.48 \\
52.62 \\
55.21 \\
52,84 \\
64.97 \\
42.26 \\
54.71 \\
57.61 \\
23.84 \\
50.46 \\
55.77 \\
55.73 \\
54.77 \\
52.50 \\
52.34 \\
56.35 \\
35.24 \\
41.37 \\
28.57\end{array}$ & $\begin{array}{r}28.20 \\
49.18 \\
43.56 \\
19.64 \\
18.69 \\
25.24 \\
31.85 \\
30.54 \\
22.98 \\
27.13 \\
17.37 \\
51.37 \\
49.50 \\
51.24 \\
52.17 \\
37.59 \\
28.16 \\
23.68 \\
31.46 \\
3.90 \\
10.94 \\
18.34 \\
16.77 \\
17.84 \\
31.00 \\
42.23 \\
42.66 \\
41.96 \\
37.53 \\
37.30 \\
30.01 \\
59.83 \\
54.10 \\
61.46\end{array}$ & $\begin{array}{r}17.41 \\
14.16 \\
12.62 \\
40.68 \\
42.03 \\
29.58 \\
12.32 \\
14.12 \\
27.55 \\
20.96 \\
41.55 \\
12.71 \\
15.99 \\
12.85 \\
10.67 \\
15.93 \\
19.22 \\
21.11 \\
15.70 \\
31.13 \\
46.80 \\
26.95 \\
25.62 \\
58.32 \\
18.54 \\
2.00 \\
1.61 \\
3.27 \\
9.97 \\
10.36 \\
13.64 \\
4.93 \\
4.53 \\
9.97\end{array}$ & $\begin{array}{r}1.33 \\
.49 \\
.50 \\
.36 \\
.32 \\
.33 \\
.27 \\
.61 \\
.38 \\
.56 \\
.61 \\
.46 \\
.60 \\
.61 \\
.72 \\
1.75 \\
.90 \\
.60 \\
.35 \\
2.39 \\
.48 \\
.41 \\
2.18 \\
.24 \\
.75 \\
.27 \\
.25 \\
.22 \\
.39 \\
.43 \\
.57 \\
.20 \\
.20 \\
.23\end{array}$ & $\begin{array}{l}1.89 \\
2.83 \\
3.00 \\
1.44 \\
1.99 \\
1.94 \\
1.96 \\
2.53 \\
2.64 \\
1.99 \\
2.20 \\
3.58 \\
3.14 \\
2.82 \\
3.16 \\
2.24 \\
1.86 \\
1.76 \\
1.97 \\
1.42 \\
1.97 \\
1.97 \\
1.69 \\
1.57 \\
3.22 \\
.90 \\
.93 \\
1.43 \\
3.51 \\
3.39 \\
3.25 \\
2.30 \\
1.96 \\
2.55\end{array}$ & $\begin{array}{r}4,293 \\
4,348 \\
4,481 \\
\ldots \ldots \ldots . \\
\ldots \ldots \ldots \\
\ldots 4,725 \\
\ldots \ldots \ldots \ldots \\
4,083 \\
4,608 \\
\ldots 4,557 \\
4,345 \\
4,579 \\
4,773 \\
4,553 \\
4,369 \\
3,943 \\
4,434 \\
\ldots \ldots \ldots\end{array}$ & 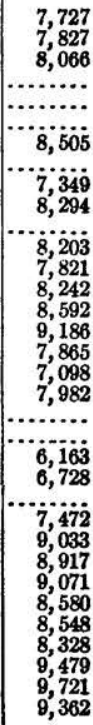 \\
\hline
\end{tabular}


[F. M. Stanton, U. S. Geological Survey, analyst.]

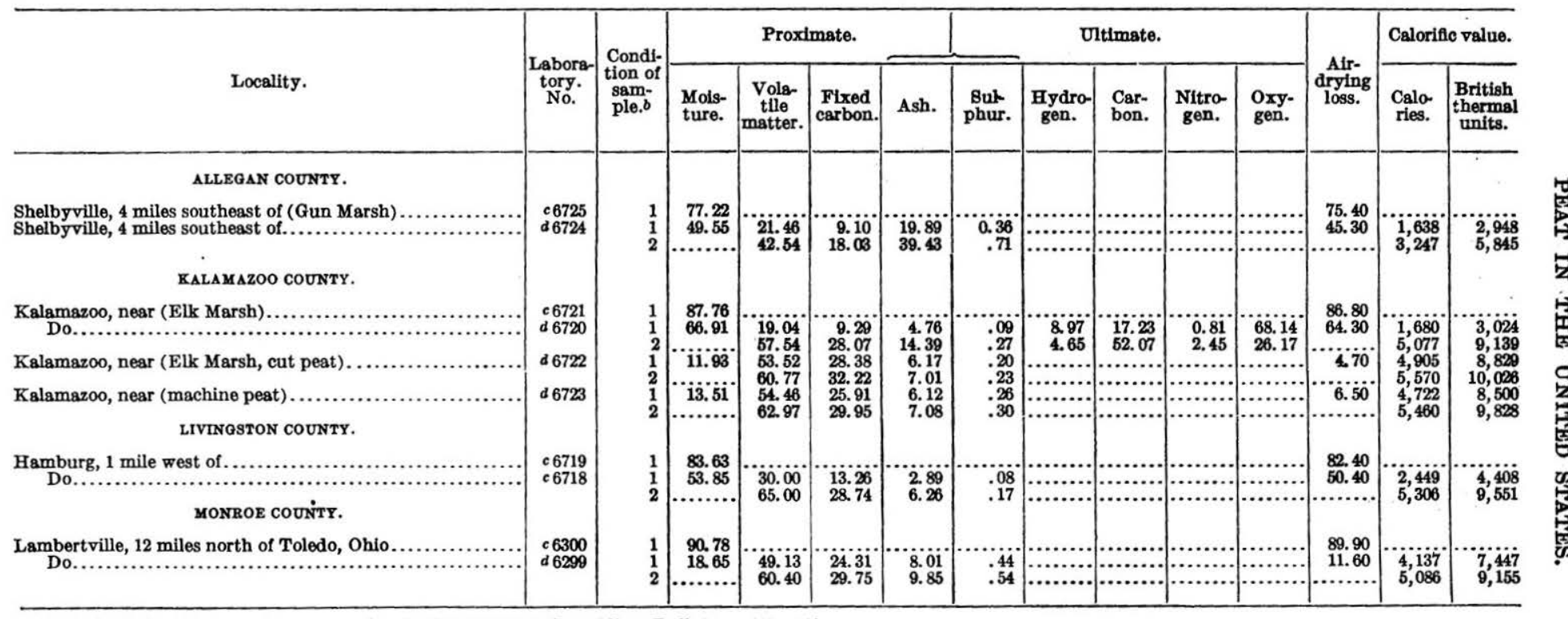

a Davis, C. A., The uses of peat for fuel and other purposes: Bur. Mines Bull. 16, p. 197, 1911. 
MINRESOTA.

Analyses of peat and muck.a

[U. S. Bureau of Mines.]

\begin{tabular}{|c|c|c|c|c|c|c|c|c|c|c|c|c|c|}
\hline \multirow{2}{*}{ County. } & \multirow{2}{*}{$\begin{array}{c}\text { Analysis } \\
\text { No. }\end{array}$} & \multirow{2}{*}{$\begin{array}{c}\text { Locality } \\
\text { No. }\end{array}$} & \multirow{2}{*}{$\begin{array}{l}\text { Moisture } \\
\text { as } \\
\text { received. }\end{array}$} & \multicolumn{3}{|c|}{ Proximate. } & \multicolumn{5}{|c|}{ Ultimate. } & \multicolumn{2}{|c|}{$\begin{array}{l}\text { Calorific value (Brit- } \\
\text { ish thermal units). }\end{array}$} \\
\hline & & & & $\begin{array}{l}\text { Volatile } \\
\text { matter. }\end{array}$ & $\begin{array}{c}\text { Fixed } \\
\text { carbon. }\end{array}$ & Ash. & Sulphur. & Nitrogen. & $\begin{array}{c}\text { Hydro- } \\
\text { gen. }\end{array}$ & Carbon. & Oxygen. & $\begin{array}{c}\text { Moisture } \\
\text { free. }\end{array}$ & $\begin{array}{l}\text { Moisture } \\
\text { and ash } \\
\text { free. }\end{array}$ \\
\hline \multirow{16}{*}{ 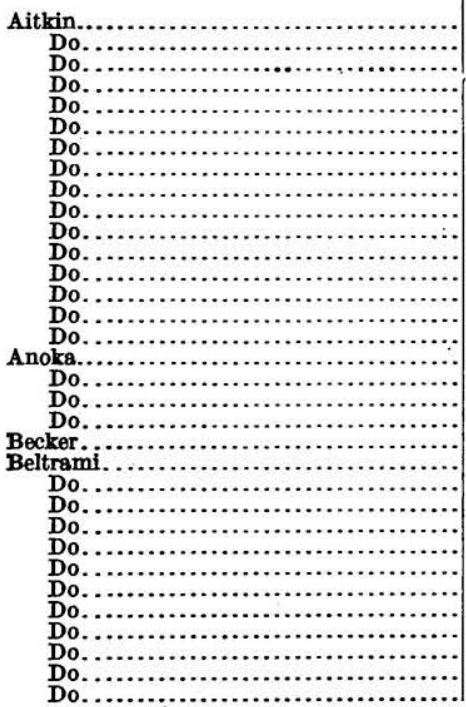 } & \multirow{16}{*}{$\begin{array}{r}1 \\
2 \\
3 \\
4 \\
5 \\
6 \\
7 \\
8 \\
9 \\
10 \\
11 \\
12 \\
13 \\
14 \\
15 \\
16 \\
17 \\
18 \\
19 \\
20 \\
21 \\
22 \\
23 \\
24 \\
25 \\
26 \\
27 \\
28 \\
29 \\
30 \\
31 \\
32 \\
33\end{array}$} & \multirow{16}{*}{$\begin{array}{r}17 \\
54 \\
153 \\
152 \\
154 \\
155 \\
156 \\
157 \\
158 \\
159 \\
160 \\
161 \\
162 \\
163 \\
164 \\
226 \\
142 \\
143 \\
144 \\
145 \\
141 \\
15 \\
197 \\
16 \\
194 \\
195 \\
196 \\
103 \\
104 \\
105 \\
106 \\
107 \\
108\end{array}$} & \multirow[b]{2}{*}{$\begin{array}{r}10.85 \\
7.75 \\
7.85 \\
7.30 \\
6.60 \\
7.95 \\
8.15 \\
7.30\end{array}$} & \multirow[b]{2}{*}{\begin{tabular}{l}
54.44 \\
\hdashline 9.70 \\
69.34 \\
67.85 \\
50.80 \\
67.30 \\
69.79 \\
68.93
\end{tabular}} & $\begin{array}{c}19.05 \\
\ldots \ldots . .\end{array}$ & $\begin{array}{c}26.51 \\
\ldots \ldots .\end{array}$ & $\begin{array}{c}0.31 \\
\ldots \ldots .\end{array}$ & $\begin{array}{c}2.61 \\
\ldots \ldots \ldots\end{array}$ & & & & $\begin{array}{c}8,134 \\
\ldots \ldots . .\end{array}$ & 10,165 \\
\hline & & & & & $\begin{array}{l}\because 23.36 \\
21.87 \\
20.98 \\
14.03 \\
18.09 \\
21.17 \\
23.73\end{array}$ & $\begin{array}{r}6.94 \\
8.79 \\
11.17 \\
35.17 \\
14.61 \\
9.04 \\
7.34\end{array}$ & $\begin{array}{r}.20 \\
.27 \\
.18 \\
. .78 \\
.18 \\
.16 \\
.20\end{array}$ & $\begin{array}{l}\ldots .01 \\
2.20 \\
2.16 \\
2.01 \\
2.23 \\
1.69 \\
1.81\end{array}$ & 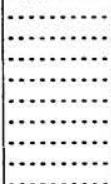 & …….... & 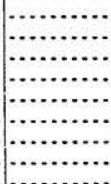 & \begin{tabular}{r}
$\dddot{9}, 537$ \\
9,156 \\
9,435 \\
\hdashline 8,518 \\
8,610 \\
9,469
\end{tabular} & $\begin{array}{r}10,098 \\
10,038 \\
10,621 \\
\cdots, 975 \\
9,975 \\
9,466 \\
10,219\end{array}$ \\
\hline & & & 7.50 & $\begin{array}{c}\dddot{6} .62 \\
\ldots \ldots \ldots\end{array}$ & 21.19 & ii.19 & .18 & 1.92 & & & & $\dddot{8}, \overline{517}$ & 90,590 \\
\hline & & & $\begin{array}{l}7.95 \\
8.90\end{array}$ & $\begin{array}{l}70.95 \\
67.62\end{array}$ & $\begin{array}{l}18.79 \\
23.10\end{array}$ & $\begin{array}{r}20.26 \\
9.28\end{array}$ &. .200 & $\begin{array}{l}\dddot{2.040} \\
2.06\end{array}$ & & & & $\begin{array}{l}7,7,00 \\
8,912\end{array}$ & $\begin{array}{r}100,7 i 3 \\
9,824\end{array}$ \\
\hline & & & $\begin{array}{r}10.40 \\
7.85\end{array}$ & 68.53 & $\begin{array}{l}21.15 \\
19.48\end{array}$ & 10.32 & .28 & 2.21 & $\cdots$ & $\cdots$ & & $\begin{array}{l}9,090 \\
9,593\end{array}$ & 10,136 \\
\hline & & & 8.35 & 72.94 & $\begin{array}{l}14.40 \\
14.89\end{array}$ & 12.17 & .27 & $\begin{array}{l}2.80 \\
2.87\end{array}$ & & (a.............. & & 8,583 & 9,772 \\
\hline & & & $\begin{array}{l}9.40 \\
8.70\end{array}$ & $\begin{array}{l}62.86 \\
59.09\end{array}$ & $\begin{array}{l}20.53 \\
15.77\end{array}$ & $\begin{array}{l}16.61 \\
25.14\end{array}$ & $\begin{array}{r}.30 \\
.30\end{array}$ & $\begin{array}{l}2.96 \\
2.88\end{array}$ & (…… & …… & & $\begin{array}{l}8,062 \\
7,456\end{array}$ & $\begin{array}{l}9,668 \\
9,960\end{array}$ \\
\hline & & & 9.00 & 61.43 & 17. 75 & 20.82 & .27 & 2.78 & ................ & $\cdots$ & ...................... & 7,976 & 10,073 \\
\hline & & & $\begin{array}{l}10.45 \\
11.18\end{array}$ & $\begin{array}{l}62.54 \\
60.63\end{array}$ & $\begin{array}{l}15.80 \\
23.33\end{array}$ & $\begin{array}{l}21.66 \\
16.04\end{array}$ & $\begin{array}{r}.95 \\
.41\end{array}$ & $\begin{array}{l}2.27 \\
1.73\end{array}$ & & & & $\begin{array}{l}9,741 \\
8,084\end{array}$ & $\begin{array}{r}12,434 \\
9,628\end{array}$ \\
\hline & & & 11.25 & 63.15 & 20.46 & 16.39 & .33 & 1.51 & …......... & .............. & an. & 7,554 & 9,035 \\
\hline & & & $\begin{array}{l}11.60 \\
11.50\end{array}$ & $\begin{array}{l}53.77 \\
64.97\end{array}$ & $\begin{array}{l}18.37 \\
22.88\end{array}$ & $\begin{array}{l}27.86 \\
12.15\end{array}$ & .24 & $\begin{array}{l}1.54 \\
2.82\end{array}$ & …… & .............. & 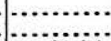 & $\begin{array}{l}6,406 \\
8,429\end{array}$ & $\begin{array}{l}8,880 \\
9,595\end{array}$ \\
\hline & & & $\begin{array}{l}12.10 \\
12.05\end{array}$ & 63.14 & 19.85 & 17.01 & $\begin{array}{r}.53 \\
.25\end{array}$ & 1.97 & 4.56 & 46.19 & 29.74 & 7,610 & ק̈ \\
\hline & & & 8.95 & 71.99 & 12.63 & 15. 38 & 1.24 & 2.49 & $\mid$ & ……............ & [............... & 10,906 & 12,8 \\
\hline & & & $\begin{array}{l}8.25 \\
7.80\end{array}$ & $\begin{array}{l}61.12 \\
59.82\end{array}$ & $\begin{array}{l}18.23 \\
13.93\end{array}$ & $\begin{array}{l}20.65 \\
26.25\end{array}$ & $\begin{array}{l}.52 \\
.84\end{array}$ & $\begin{array}{l}2.21 \\
1.98\end{array}$ & …… & ……, & $\ldots \ldots \ldots$ & $\begin{array}{l}7,716 \\
7,072\end{array}$ & $\begin{array}{l}9,724 \\
9,589\end{array}$ \\
\hline & & & 6.15 & 43.95 & 10.34 & 45.71 & $\because \because$ & 1.60 & ............. & & & $\cdots$ & 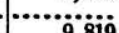 \\
\hline & & & $\begin{array}{l}7.90 \\
7.60\end{array}$ & $\begin{array}{l}59.59 \\
51.01\end{array}$ & $\begin{array}{l}18.86 \\
15.44\end{array}$ & $\begin{array}{l}21.55 \\
33.55\end{array}$ & 1.01 & $\begin{array}{l}2.31 \\
1.84\end{array}$ & & & & 7,703 & 9,8 \\
\hline
\end{tabular}


Analyses of peat and muck-Continued.

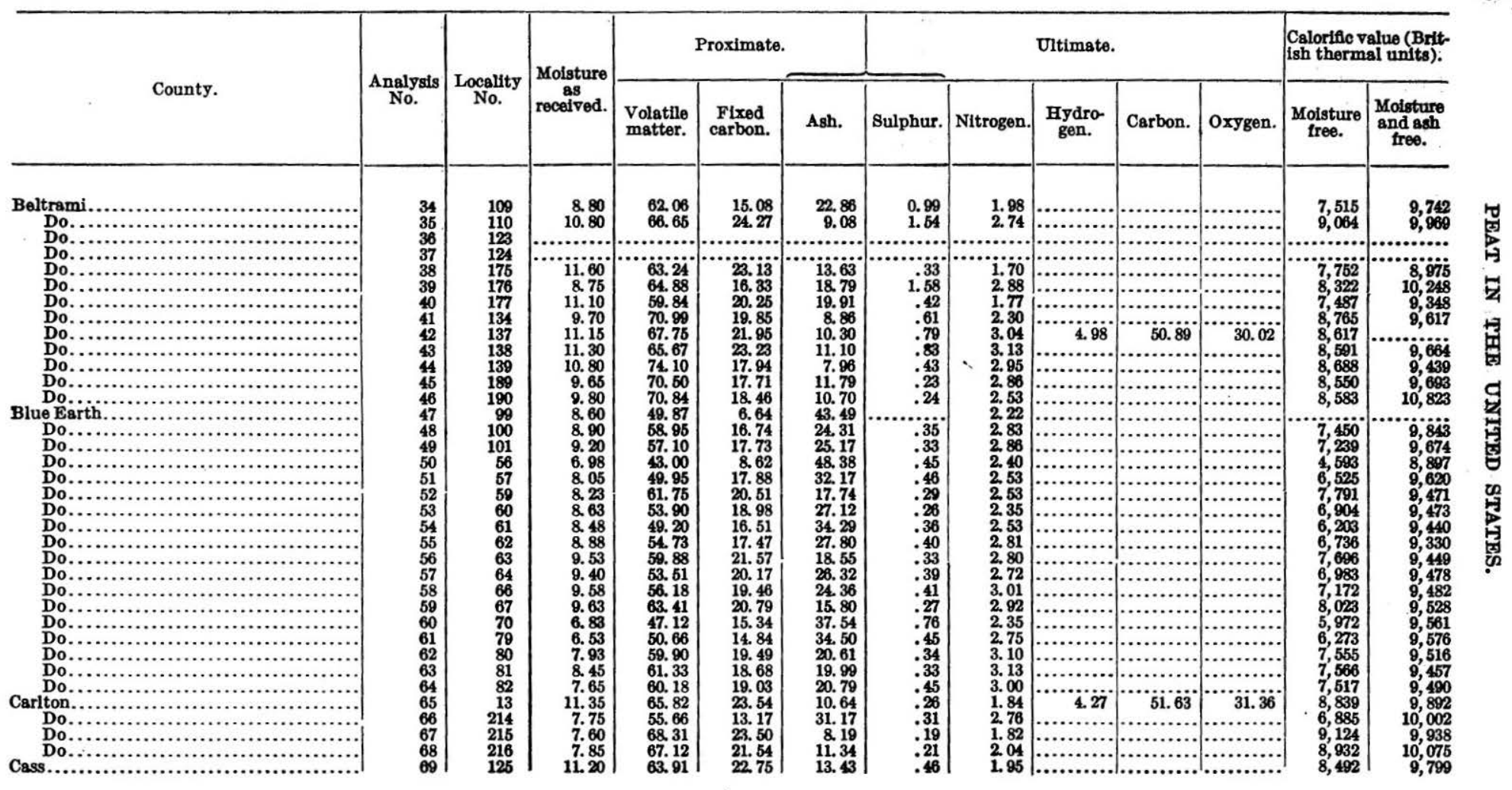




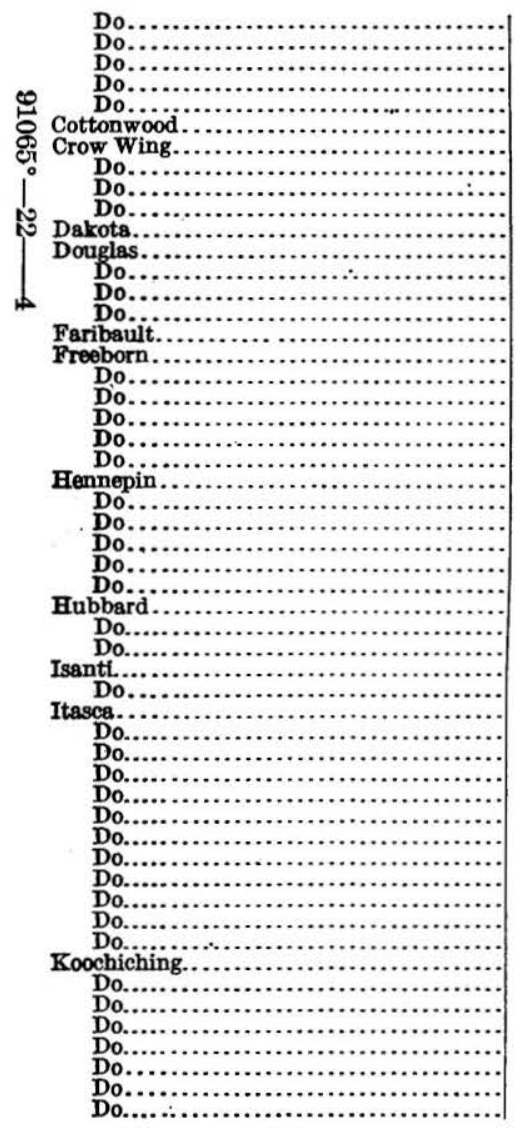

\begin{tabular}{|c|c|c|c|c|c|c|}
\hline & $\begin{array}{r}150 \\
151 \\
166 \\
167 \\
111 \\
42 \\
43 \\
55\end{array}$ & $\begin{array}{r}8.10 \\
8.45 \\
10.70 \\
9.45 \\
7.10 \\
10.15 \\
9.78 \\
8.75\end{array}$ & $\begin{array}{l}49.51 \\
69.58 \\
66.01 \\
73.72 \\
41.60 \\
64.75 \\
65.51\end{array}$ & $\begin{array}{r}15.67 \\
20.81 \\
18.59 \\
18.55 \\
3.34 \\
24.45 \\
24.20\end{array}$ & $\begin{array}{r}34.82 \\
9.61 \\
15.40 \\
7.73 \\
55.06 \\
10.80 \\
10.29\end{array}$ & י.219 \\
\hline & $\begin{array}{l}165 \\
253 \\
130\end{array}$ & $\begin{array}{l}7.80 \\
9.00 \\
9.10\end{array}$ & $\begin{array}{l}61.23 \\
68.02 \\
59.74\end{array}$ & $\begin{array}{l}17.5 \mathrm{i} \\
19.40 \\
11.71\end{array}$ & $\begin{array}{l}21.26 \\
12.58 \\
128.55\end{array}$ & i. 13 \\
\hline & $\begin{array}{l}\mathbf{1 3 1} \\
132 \\
133\end{array}$ & $\begin{array}{l}10.80 \\
10.05 \\
11.15\end{array}$ & $\begin{array}{l}64.07 \\
57.14 \\
66.63\end{array}$ & $\begin{array}{l}18.78 \\
17.57 \\
16.94\end{array}$ & $\begin{array}{l}17.15 \\
25.29 \\
16.43\end{array}$ & .38 \\
\hline & $\begin{array}{l}102 \\
94 \\
948\end{array}$ & $\begin{array}{l}7.40 \\
7.05 \\
7.55\end{array}$ & $\begin{array}{l}46.14 \\
41.90 \\
41.81\end{array}$ & $\begin{array}{l}4.08 \\
9.90 \\
9.62\end{array}$ & $\begin{array}{l}49.78 \\
48.20 \\
48.57\end{array}$ & "ij \\
\hline & $\begin{array}{l}94 \mathrm{~b} \\
95 \\
96 \\
97\end{array}$ & $\begin{array}{l}5.55 \\
8.45 \\
6.50 \\
9.25\end{array}$ & $\begin{array}{l}26.20 \\
42.85 \\
34.22 \\
52.29\end{array}$ & $\begin{array}{r}4.93 \\
8.16 \\
5.94 \\
51.94\end{array}$ & $\begin{array}{l}68.87 \\
489 \\
59.84 \\
33.74\end{array}$ & \\
\hline & $\begin{array}{l}246 \\
247\end{array}$ & $\begin{array}{l}8.50 \\
8.55 \\
8.60\end{array}$ & $\begin{array}{l}62.00 \\
71.01\end{array}$ & $\begin{array}{l}17.66 \\
17.67\end{array}$ & $\begin{array}{r}20.34 \\
\times \quad 11.32 \\
\end{array}$ & $\because 6 \mathrm{i}$ \\
\hline & 24 & $\begin{array}{r}9.05 \\
10.10\end{array}$ & $\begin{array}{l}67 . \\
72 . \\
67\end{array}$ & $\begin{array}{l}15.29 \\
15.97 \\
110.95\end{array}$ & $\begin{array}{l}16.82 \\
12.01 \\
12\end{array}$ & $\begin{array}{l}.21 \\
.46\end{array}$ \\
\hline & & $\begin{array}{r}9.10 \\
9.10 \\
12.70\end{array}$ & $\begin{array}{l}7.0 . \\
72 .\end{array}$ & 15.79 & $\begin{array}{l}11.32 \\
11.55 \\
21.36\end{array}$ & $\begin{array}{r}.61 \\
.36 \\
.19\end{array}$ \\
\hline & & $\begin{array}{l}9.30 \\
\end{array}$ & & $\begin{array}{l}21.00 \\
21.45\end{array}$ & $\begin{array}{l}14.66 \\
14.60\end{array}$ & $\begin{array}{l}.156 \\
.56\end{array}$ \\
\hline & 244 & 8.40 & $\begin{array}{l}60 . \\
68 .\end{array}$ & 20 . & $\begin{array}{r}9.88 \\
10.48\end{array}$ & .28 \\
\hline & & $\begin{array}{l}7.70 \\
8.35\end{array}$ & ${ }_{60}^{73}$ & 20 & $\begin{array}{r}6.28 \\
19.91\end{array}$ & $\begin{array}{l}.25 \\
.23\end{array}$ \\
\hline & & 8. & & & 13.05 & .24 \\
\hline & & 9 & & & 9.98 & .23 \\
\hline & & 10 & & 17. & $\begin{array}{l}10.88 \\
13.31\end{array}$ & 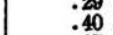 \\
\hline & & 11. & & & & 17 \\
\hline & & & & & $\begin{array}{l}21.18 \\
11.5\end{array}$ & .20 \\
\hline & & & & & & .21 \\
\hline & & & & & $\begin{array}{r}11.24 \\
9.24\end{array}$ & .260 \\
\hline & & & & & & .58 \\
\hline & 12 & $\begin{array}{r}7.80 \\
11.05\end{array}$ & & & $\begin{array}{l}\begin{array}{l}6.48 \\
13.88\end{array}\end{array}$ & 1.33 \\
\hline & & 10.15 & & & 8.79 & .75 \\
\hline & & 11. & & & 11. & .24 \\
\hline & & 8. & & & 11. & .21 \\
\hline & & 9.95 & 68.68 & 22.21 & 9.16 & .22 \\
\hline
\end{tabular}

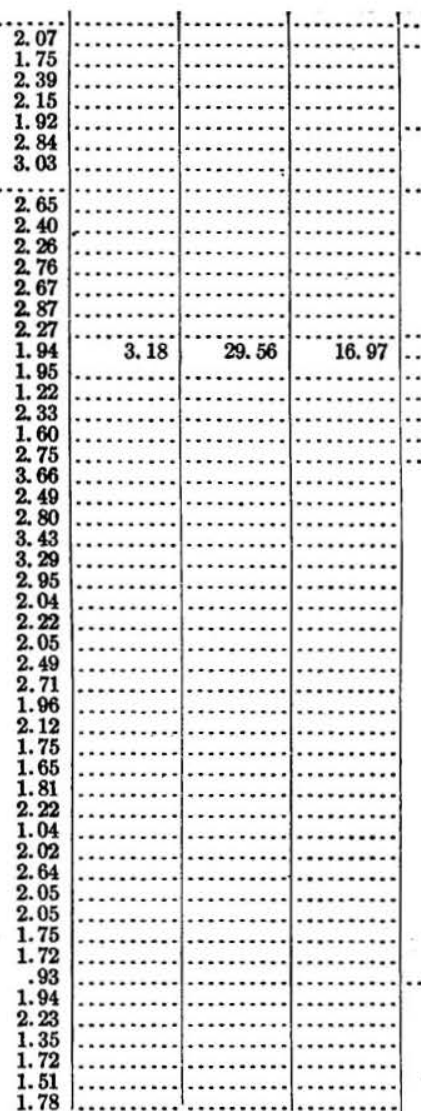

\begin{tabular}{|c|c|}
\hline $\begin{array}{r}8,427 \\
7,923 \\
9,042\end{array}$ & $\begin{array}{l}9,323 \\
9,365 \\
9,800\end{array}$ \\
\hline$\dddot{8} 8,859$ & $\dddot{9} 97,589$ \\
\hline 7,456 & $\dddot{9}, \dddot{3} 350$ \\
\hline $\begin{array}{l}7,909 \\
7,173 \\
8,170\end{array}$ & $\begin{array}{l}9,609 \\
9,601 \\
9,752\end{array}$ \\
\hline & $\cdots$ \\
\hline & 羊 \\
\hline 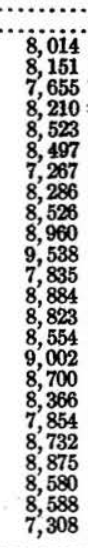 & 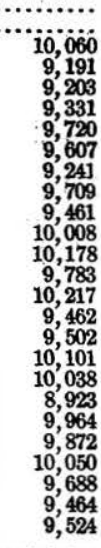 \\
\hline $\begin{array}{l}8,192 \\
8,640 \\
7,753 \\
8,146 \\
8,394 \\
8,406\end{array}$ & $\begin{array}{l}\dddot{9}, 5,3 i 2 \\
9,473 \\
8,739 \\
9,217 \\
9,507 \\
9,254\end{array}$ \\
\hline
\end{tabular}


Analyses of peat and muck-Continued.

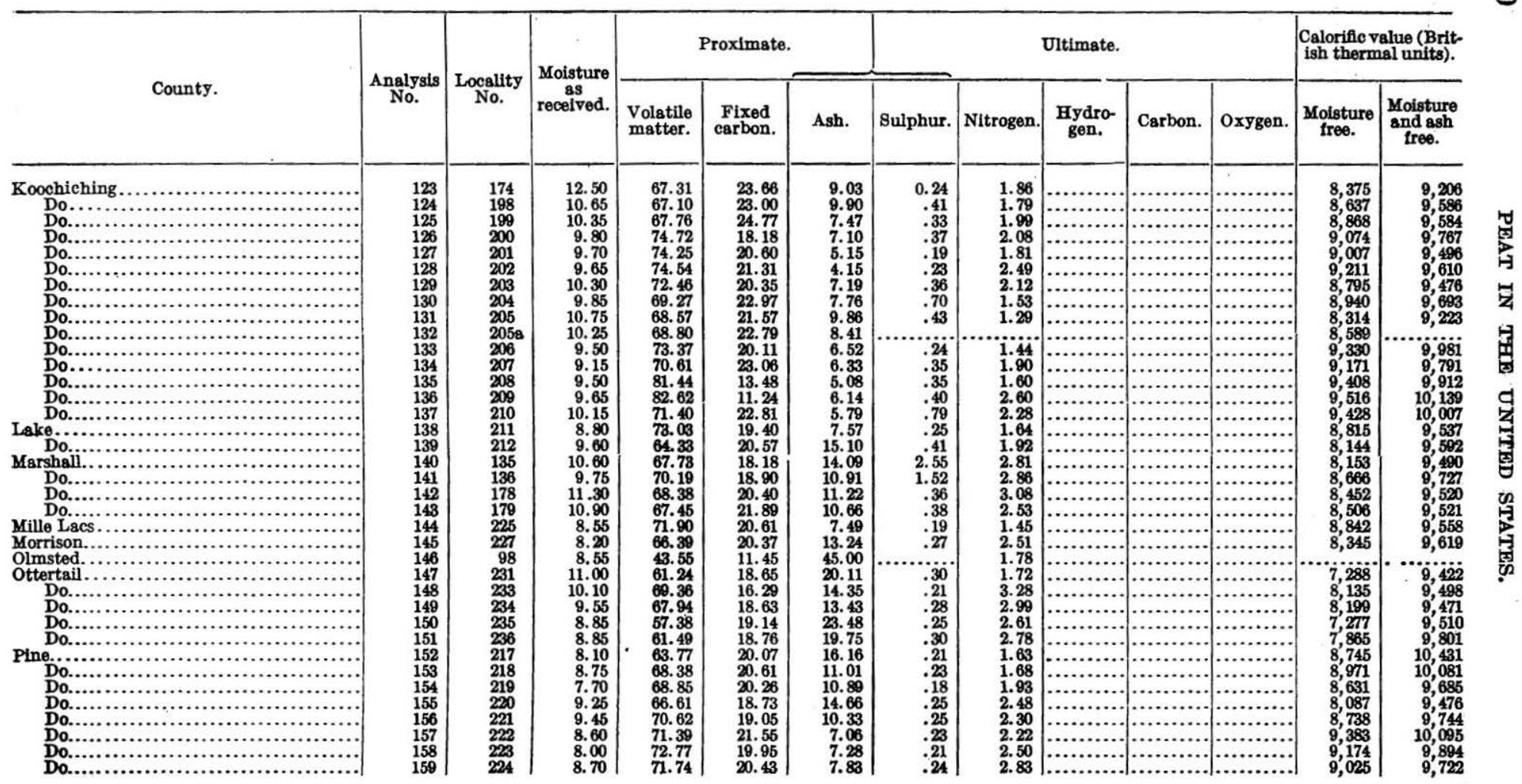




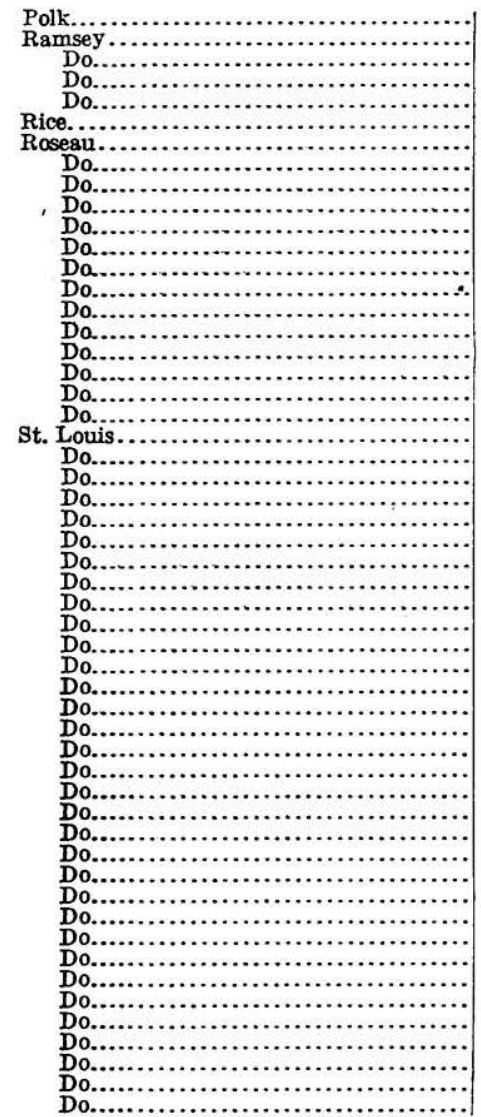


Analyses of peat and muck-Continued.

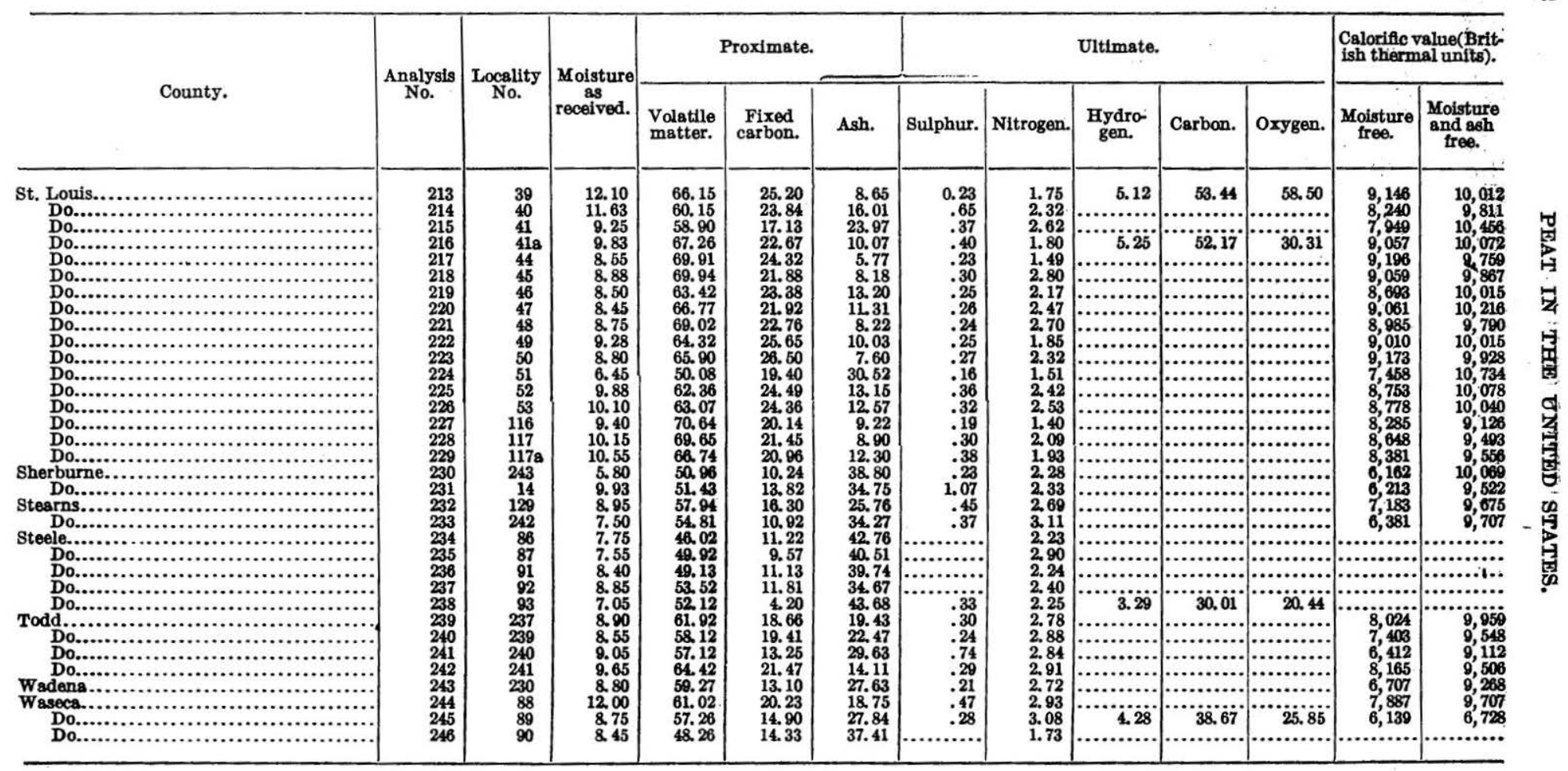


NEW HAMPGEMRE.

Analyses of moisture-free peat and muck.

[H. M. Cooper, Bureau of Mines, analyst.]

\begin{tabular}{|c|c|c|c|c|c|c|c|c|c|c|c|c|}
\hline \multirow{2}{*}{$\begin{array}{l}\text { Local- } \\
\text { ity } \\
\text { No. }\end{array}$} & \multirow[b]{2}{*}{ County. } & \multirow{2}{*}{$\begin{array}{l}\text { Bam- } \\
\text { ple } \\
\text { and } \\
\text { anal- } \\
\text { ysls } \\
\text { No. }\end{array}$} & \multicolumn{3}{|c|}{ Proximate. } & \multicolumn{5}{|c|}{ Ultimate. } & \multicolumn{2}{|c|}{ Calorific value. } \\
\hline & & & $\begin{array}{l}\text { Vola- } \\
\text { tile } \\
\text { mat- } \\
\text { ter. }\end{array}$ & $\begin{array}{c}\text { Fixed } \\
\text { car- } \\
\text { bon. }\end{array}$ & Ash. & $\begin{array}{l}\text { Sul- } \\
\text { phur. }\end{array}$ & $\begin{array}{c}\text { Hydro- } \\
\text { gen. }\end{array}$ & $\begin{array}{l}\text { Car- } \\
\text { bon. }\end{array}$ & $\begin{array}{l}\text { Nitro- } \\
\text { gen. }\end{array}$ & $\begin{array}{l}\text { Oxy- } \\
\text { gen. }\end{array}$ & $\begin{array}{l}\text { Calo- } \\
\text { ries. }\end{array}$ & $\begin{array}{c}\text { British } \\
\text { ther- } \\
\text { mal } \\
\text { units. }\end{array}$ \\
\hline $\begin{array}{r}1 \\
2 \\
5 \\
6 \\
7 \\
8 \\
10 \\
16\end{array}$ & 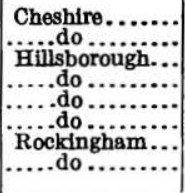 & $\begin{array}{l}7 \\
8 \\
3 \\
4 \\
5 \\
6 \\
1 \\
2\end{array}$ & $\begin{array}{l}35.18 \\
51.02 \\
44.36 \\
28.41 \\
35.00 \\
43.58 \\
17.59 \\
68.50\end{array}$ & $\begin{array}{r}49.88 \\
45.00 \\
50.28 \\
34.17 \\
33.25 \\
35.54 \\
9.38 \\
26.40\end{array}$ & $\begin{array}{r}14.94 \\
3.98 \\
5.36 \\
37.42 \\
31.75 \\
20.88 \\
73.03 \\
5.10\end{array}$ & $\begin{array}{r}0.29 \\
.16 \\
.20 \\
.34 \\
.32 \\
.31 \\
1.54 \\
.37\end{array}$ & 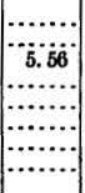 & 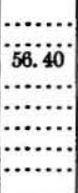 & $\begin{array}{l}1.60 \\
1.76 \\
2.03 \\
1.44 \\
1.97 \\
1.82 \\
1.10 \\
1.69\end{array}$ & 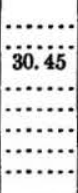 & \begin{tabular}{c}
4,654 \\
5,333 \\
5,444 \\
\hdashline$\ldots \ldots$ \\
\hdashline 4,109 \\
\hdashline 5,648
\end{tabular} & $\begin{array}{r}8,377 \\
9,599 \\
9,800 \\
\cdots \ldots . . \\
\hdashline 7,396 \\
\hdashline 10,166\end{array}$ \\
\hline
\end{tabular}

Analyses of peat and muck.

[F. M. Stanton, U. S. Geological Survey, analyst.]

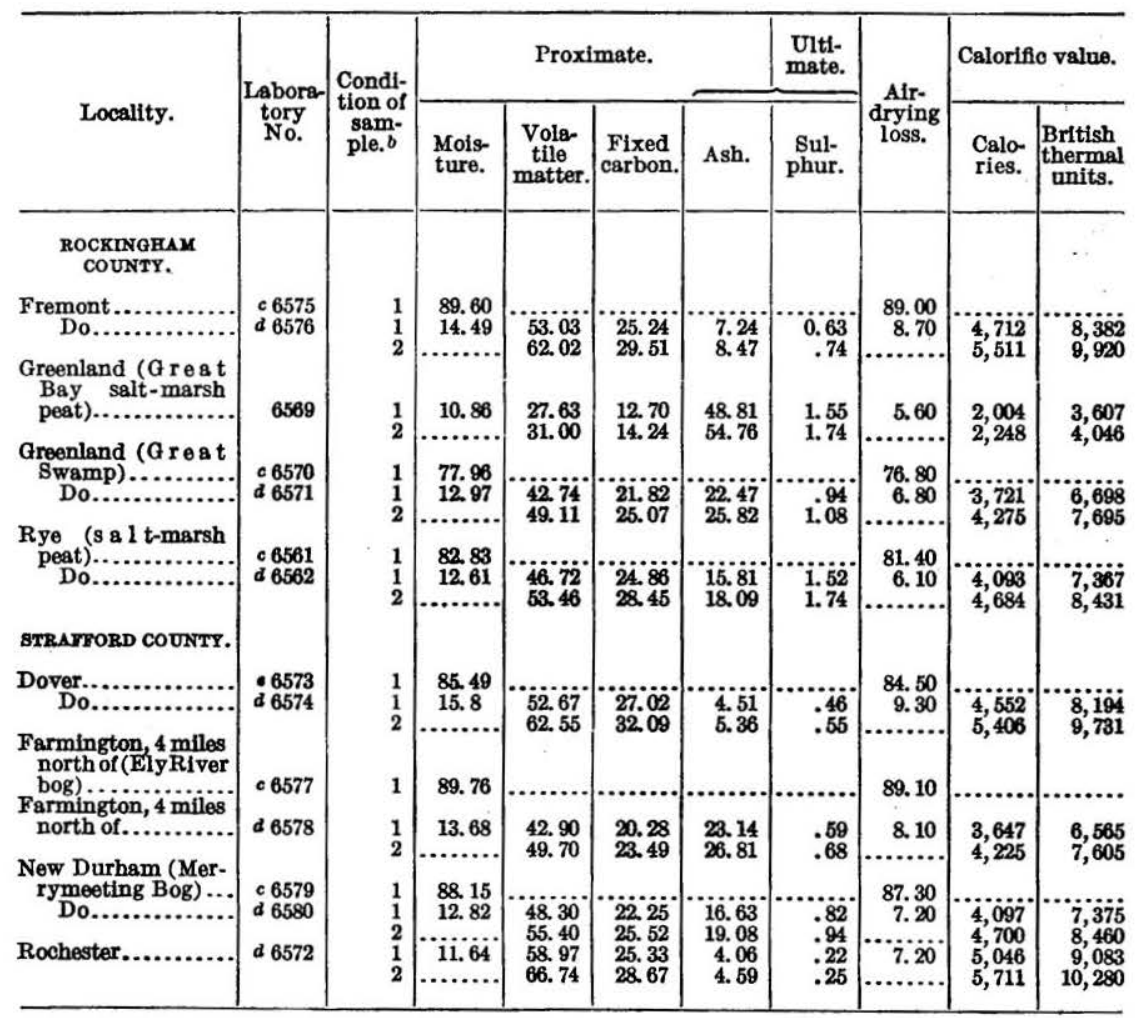

a Dayis, C. A., The uses of pest for fuel and other purposes: Bur. Mines Bull. 16, pp. 197-198, 1911. b 1, Native peat. 2, Moisture-free peat.

c Bottle sample.

d Sack sample. 
NEW JERSEY.

Analyses of moisture-free peat.

[H. M. Cooper, Bureau of Mines, analyst.]

\begin{tabular}{|c|c|c|c|c|c|c|c|c|}
\hline \multirow[b]{2}{*}{, } & \multirow{2}{*}{$\begin{array}{c}\text { Sample } \\
\text { and } \\
\text { analysis } \\
\text { No. }\end{array}$} & \multicolumn{3}{|c|}{ Proximate. } & \multicolumn{2}{|c|}{ Ultimate. } & \multicolumn{2}{|c|}{ Calorifle value. } \\
\hline & & $\begin{array}{l}\text { Volatile } \\
\text { matter. }\end{array}$ & $\begin{array}{l}\text { Fixed } \\
\text { carbon. }\end{array}$ & Ash. & Sulphur. & Nitrogen. & Calories. & $\begin{array}{l}\text { British } \\
\text { thermal } \\
\text { units. }\end{array}$ \\
\hline SUSSEX COUNTY. & & & & & & & & \\
\hline 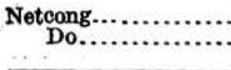 & $\mathbf{1}$ & $\begin{array}{l}57.76 \\
55.51\end{array}$ & $\begin{array}{l}33.84 \\
37.44\end{array}$ & $\begin{array}{l}8.90 \\
7.05\end{array}$ & $\begin{array}{r}0.44 \\
.49\end{array}$ & $\begin{array}{l}1.87 \\
2.88\end{array}$ & $\begin{array}{r}5,068 \\
5,038\end{array}$ & $\begin{array}{l}9,122 \\
9,068\end{array}$ \\
\hline
\end{tabular}

Analyses of high-grade air-dried peat."

\begin{tabular}{|c|c|c|c|c|c|c|}
\hline Locality (nearest town). & $\begin{array}{c}\text { Locality } \\
\text { number } \\
\text { (P1. } \\
\text { XII). }\end{array}$ & $\begin{array}{c}\text { Sample } \\
\text { and } \\
\text { analysis } \\
\text { No. }\end{array}$ & Ash. & Nitrogen. & Calories. & Coke. \\
\hline 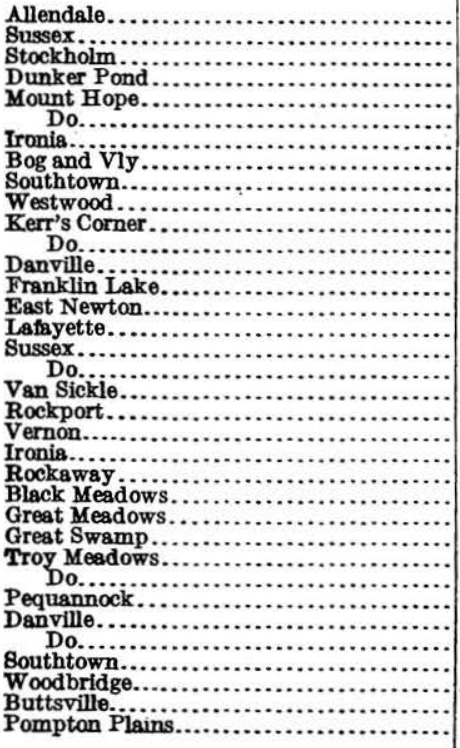 & $\begin{array}{r}5 \\
20 \\
26 \\
26 \\
29 \\
29 \\
32 \\
47 \\
58 \\
4 \\
58 \\
58 \\
56 \\
63 \\
12 \\
15 \\
19 \\
25 \\
22 \\
22 \\
23 \\
32 \\
33 \\
37 \\
37 \\
38 \\
41 \\
41 \\
45 \\
56 \\
56 \\
58\end{array}$ & $\begin{array}{r}3 \\
44 \\
58 \\
59 \\
68 \\
70 \\
72 \\
102 \\
112 \\
115 \\
117 \\
118 \\
119 \\
130 \\
15 \\
22 \\
34 \\
46 \\
47 \\
48 \\
54 \\
73 \\
75 \\
83 \\
84 \\
89 \\
92 \\
93 \\
98 \\
110 \\
111 \\
116 \\
125 \\
128 \\
\end{array}$ & $\begin{array}{r}5.04 \\
7.27 \\
5.75 \\
6.24 \\
5.14 \\
9.47 \\
9.34 \\
7.28 \\
6.54 \\
7.30 \\
8.01 \\
7.72 \\
8.62 \\
8.34 \\
10.34 \\
10.45 \\
11.38 \\
14.59 \\
13.04 \\
11.36 \\
14.40 \\
14.26 \\
10.54 \\
12.43 \\
10.84 \\
13.86 \\
10.60 \\
13.44 \\
12.69 \\
11.04 \\
13.31 \\
13.42 \\
10.40 \\
10.41 \\
13.64\end{array}$ & $\begin{array}{l}2.17 \\
2.10 \\
2.10 \\
2.11 \\
1.27 \\
1.87 \\
1.17 \\
2.16 \\
1.62 \\
2.11 \\
2.35 \\
1.59 \\
2.45 \\
2.03 \\
2.24 \\
2.83 \\
2.34 \\
2.13 \\
2.25 \\
2.02 \\
2.73 \\
1.30 \\
2.11 \\
2.05 \\
1.88 \\
2.07 \\
1.46 \\
1.98 \\
1.61 \\
1.98 \\
1.96 \\
2.52 \\
2.62 \\
2.65 \\
2.11\end{array}$ & $\begin{array}{l}5,876 \\
4,589 \\
5,284 \\
5,265 \\
5,645 \\
5,159 \\
5,488 \\
5,378 \\
5,098 \\
5,203 \\
5,120 \\
4,982 \\
4,953 \\
5,521 \\
5,187 \\
5,004 \\
4,946 \\
4,878 \\
4,986 \\
4,908 \\
4,707 \\
5,193 \\
5,057 \\
4,791 \\
4,885 \\
4,947 \\
5,272 \\
4,901 \\
4,789 \\
5,234 \\
4,562 \\
4,795 \\
4,926 \\
4,863 \\
4,767\end{array}$ & $\begin{array}{l}29.20 \\
36.11 \\
35.31 \\
34.30 \\
43.85 \\
38.76 \\
37.64 \\
37.08 \\
34.30 \\
33.51 \\
32.62 \\
36.70 \\
33.14 \\
36.28 \\
33.09 \\
34.91 \\
37.95 \\
39.77 \\
40.01 \\
37.53 \\
36.92 \\
41.78 \\
32.25 \\
38.81 \\
37.45 \\
40.09 \\
37.80 \\
38.96 \\
39.15 \\
36.90 \\
40.26 \\
36.83 \\
34.40 \\
34.51 \\
41.55\end{array}$ \\
\hline
\end{tabular}

a Parmelee, C. W.; and McCourt, W. E., A report on the peat deposits of northern New Jersey: New Jersey Geol. Survey Ann. Rept. for 1905, p.261, 1905. 
Analyses of low-grade air-dried peat and muck."

\begin{tabular}{|c|c|c|c|c|c|}
\hline Locality (nearest town). & $\begin{array}{l}\text { Locality } \\
\text { No. } \\
\text { (Pl. XII). }\end{array}$ & $\begin{array}{c}\text { Sample } \\
\text { and } \\
\text { analysis } \\
\text { No. }\end{array}$ & Ash. & Calories. & Coke. \\
\hline 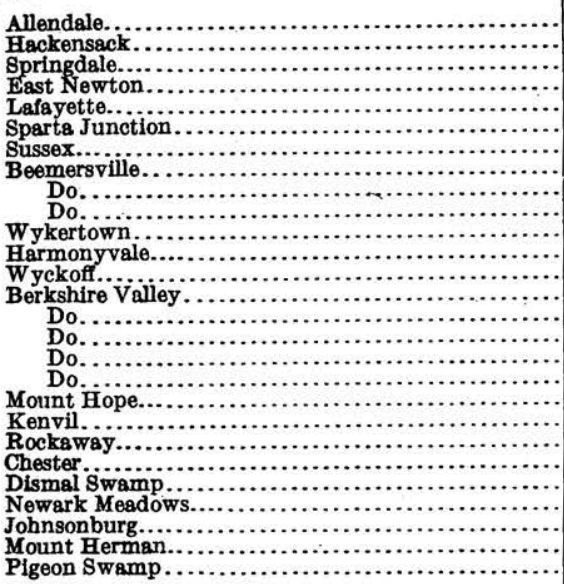 & $\begin{array}{r}5 \\
9 \\
10 \\
12 \\
15 \\
15 \\
19 \\
21 \\
21 \\
21 \\
21 \\
25 \\
27 \\
27 \\
28 \\
28 \\
28 \\
28 \\
29 \\
31 \\
33 \\
32 \\
50 \\
53 \\
57 \\
60 \\
62\end{array}$ & $\begin{array}{r}2 \\
9 \\
11 \\
16 \\
19 \\
23 \\
31 \\
35 \\
36 \\
40 \\
42 \\
57 \\
60 \\
61 \\
63 \\
64 \\
65 \\
66 \\
67 \\
71 \\
76 \\
94 \\
105 \\
108 \\
120 \\
124 \\
126\end{array}$ & $\begin{array}{l}18.89 \\
20.74 \\
24.43 \\
23.78 \\
23.29 \\
20.88 \\
20.11 \\
17.23 \\
20.82 \\
23.03 \\
22.81 \\
23.25 \\
24.70 \\
18.21 \\
24.95 \\
23.24 \\
20.10 \\
24.38 \\
16.44 \\
19.27 \\
16.46 \\
17.61 \\
20.43 \\
20.06 \\
15.82 \\
16.71 \\
20.40\end{array}$ & $\begin{array}{l}4,388 \\
4,312 \\
4,041 \\
4,212 \\
4,092 \\
4,026 \\
4,165 \\
4,814 \\
4,199 \\
4,212 \\
4,377 \\
4,027 \\
4,049 \\
4,387 \\
4,276 \\
4,456 \\
4,800 \\
4,300 \\
5,117 \\
4,285 \\
5,049 \\
4,575 \\
4,335 \\
4,347 \\
4,317 \\
4,568 \\
4,539\end{array}$ & \begin{tabular}{r}
41.57 \\
46.75 \\
\hdashline 43.73 \\
44.85 \\
40.20 \\
41.90 \\
41.40 \\
39.38 \\
44.35 \\
44.18 \\
42.60 \\
45.90 \\
46.26 \\
47.10 \\
46.01 \\
41.95 \\
45.20 \\
42.65 \\
42.94 \\
41.10 \\
42.29 \\
42.35 \\
43.55 \\
36.35 \\
36.46 \\
37.25
\end{tabular} \\
\hline
\end{tabular}

a Parmelee, C. W., and McCourt, W. E., A report on the peat deposits of northern New Jersey: New Jersey Geol. Survey Ann. Rept. for 1905, p. 262, 1905.

\section{MEW YORK.}

Analyses of moisture-free peat and muck.

[H. M. Cooper, Bureau of Mines, analyst.]

\begin{tabular}{|c|c|c|c|c|c|c|c|c|c|c|c|c|}
\hline \multirow{2}{*}{$\begin{array}{l}\text { Lo- } \\
\text { cal- } \\
\text { ity } \\
\text { No. }\end{array}$} & \multirow[b]{2}{*}{ County. } & \multirow{2}{*}{$\begin{array}{c}\text { Sam- } \\
\text { ple } \\
\text { and } \\
\text { angl- } \\
\text { ysis } \\
\text { No. }\end{array}$} & \multicolumn{3}{|c|}{ Proximate. } & \multicolumn{5}{|c|}{ Ultimate. } & \multicolumn{2}{|c|}{$\begin{array}{c}\text { Calorific } \\
\text { value. }\end{array}$} \\
\hline & & & $\begin{array}{l}\text { Vola- } \\
\text { tile } \\
\text { mat- } \\
\text { ter. }\end{array}$ & $\begin{array}{c}\text { Fixed } \\
\text { car- } \\
\text { bon. }\end{array}$ & Ash. & $\begin{array}{c}\text { Sul- } \\
\text { phur. }\end{array}$ & $\begin{array}{l}\text { Hy- } \\
\text { dro- } \\
\text { gen. }\end{array}$ & $\begin{array}{l}\text { Car- } \\
\text { bon. }\end{array}$ & $\begin{array}{l}\text { Nitro- } \\
\text { gen. }\end{array}$ & $\begin{array}{l}\text { Oxy- } \\
\text { gen. }\end{array}$ & $\begin{array}{l}\text { Calo- } \\
\text { ries. }\end{array}$ & $\begin{array}{c}\text { British } \\
\text { ther- } \\
\text { mal } \\
\text { units. }\end{array}$ \\
\hline 2 & Clinton & 18 & 43.45 & 32.78 & 23.77 & 0.31 & & & 2.53 & & 3,701 & 6,662 \\
\hline $\begin{array}{l}3 \\
5\end{array}$ & Dutche & $\begin{array}{l}19 \\
74\end{array}$ & $\begin{array}{l}33.68 \\
59.22\end{array}$ & $\begin{array}{l}26.65 \\
33.39\end{array}$ & $\begin{array}{r}39.67 \\
7.39\end{array}$ & $\begin{array}{l}.28 \\
.44\end{array}$ & 4.78 & 45.41 & $\begin{array}{l}1.86 \\
1.72\end{array}$ & 40.26 & 5,106 & 9,190 \\
\hline $\mathbf{5}$ & ….do. & 748 & 60.74 & 35.50 & 3. 76 & .22 & 5.16 & 54.97 & 1.57 & 34.32 & 5,324 & 9,583 \\
\hline $\begin{array}{l}9 \\
9\end{array}$ & Essex.... & 20 & $\begin{array}{l}36.04 \\
45.06\end{array}$ & $\begin{array}{l}53.55 \\
48.07\end{array}$ & $\begin{array}{r}10.41 \\
6.87\end{array}$ & $\begin{array}{r}.74 \\
36\end{array}$ & ....... & ........ & $\begin{array}{l}3.70 \\
3.00\end{array}$ & ........ & $\begin{array}{l}4,748 \\
4,828\end{array}$ & 8,547 \\
\hline 10 & Essex & & & & & & & & & & & \\
\hline 10 & Franklin.. & 22 & $\begin{array}{l}\begin{array}{l}11.40 \\
45.55\end{array} \\
\end{array}$ & 43.30 & $\begin{array}{l}0.04 \\
11.15\end{array}$ & $\begin{array}{l}.00 \\
.36\end{array}$ & & & 1.70 & & & $\begin{array}{l}9,125 \\
8,752\end{array}$ \\
\hline 13 & .....do... & 23 & 57.67 & 37.3 & 4.94 & .22 & ...... & .......... & 2.34 & .......... & 5,534 & 9,962 \\
\hline $\begin{array}{l}15 \\
15\end{array}$ & …..do. & 24 & 43.08 & 47.8 & 9.10 & .41 & & ........ & 2.37 & $\ldots .$. & 5,094 & 9,169 \\
\hline 16 & Genesee and & & $40.3^{2}>$ & 41. & 11.56 & .00 & & & 2.00 & & & 8,874 \\
\hline & Orle & 69 & 63.72 & 21.91 & 14.37 & 1.43 & & & 3.01 & & 4,402 & 7,923 \\
\hline $\begin{array}{l}16 \\
24\end{array}$ & Önondo..... & $\begin{array}{l}70 \\
64\end{array}$ & $\begin{array}{l}59.76 \\
61.59\end{array}$ & $\begin{array}{l}26.43 \\
31.07\end{array}$ & $\begin{array}{r}13.81 \\
7.34\end{array}$ & $\begin{array}{r}1.45 \\
.39\end{array}$ & $\begin{array}{l}3.56 \\
4.74\end{array}$ & $\begin{array}{l}46.94 \\
54.07\end{array}$ & $\begin{array}{l}2.87 \\
2.02\end{array}$ & $\begin{array}{l}31.37 \\
31.44\end{array}$ & $\begin{array}{l}4,489 \\
5,142\end{array}$ & $\begin{array}{l}8,080 \\
9,256\end{array}$ \\
\hline 24 & do & 65 & 62.01 & 30.14 & 7.85 & .44 & & ......... & 2.16 & & 4,976 & 8,957 \\
\hline $\begin{array}{l}25 \\
26\end{array}$ & $\ldots$..d & 66 & & 29.8 & 9.98 & 1.45 & $\ldots$. & …..... & & …..... & 4,743 & 8,537 \\
\hline 27 & .....do.. & 72 & 59.87 & 26.45 & 13.68 & $\begin{array}{r}.87 \\
1.48\end{array}$ & 4.74 & 49.131 & 2.89 & 27.70 & $\begin{array}{l}4,401 \\
4,651\end{array}$ & 8,372 \\
\hline $\begin{array}{l}27 \\
30\end{array}$ & seneca & 73 & & 22.21 & 23. & 1.69 & 4. 27 & 35.85 & 2.46 & 32.53 & 4,147 & 7,465 \\
\hline 31 & Wa & 67 & $\begin{array}{l}64.50 \\
50\end{array}$ & 2.68 & 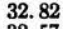 & 1.38 & & & & & & \\
\hline & & & & & & 2.10 & & & 2.59 & & & \\
\hline
\end{tabular}


Analyses of peat and muck.a

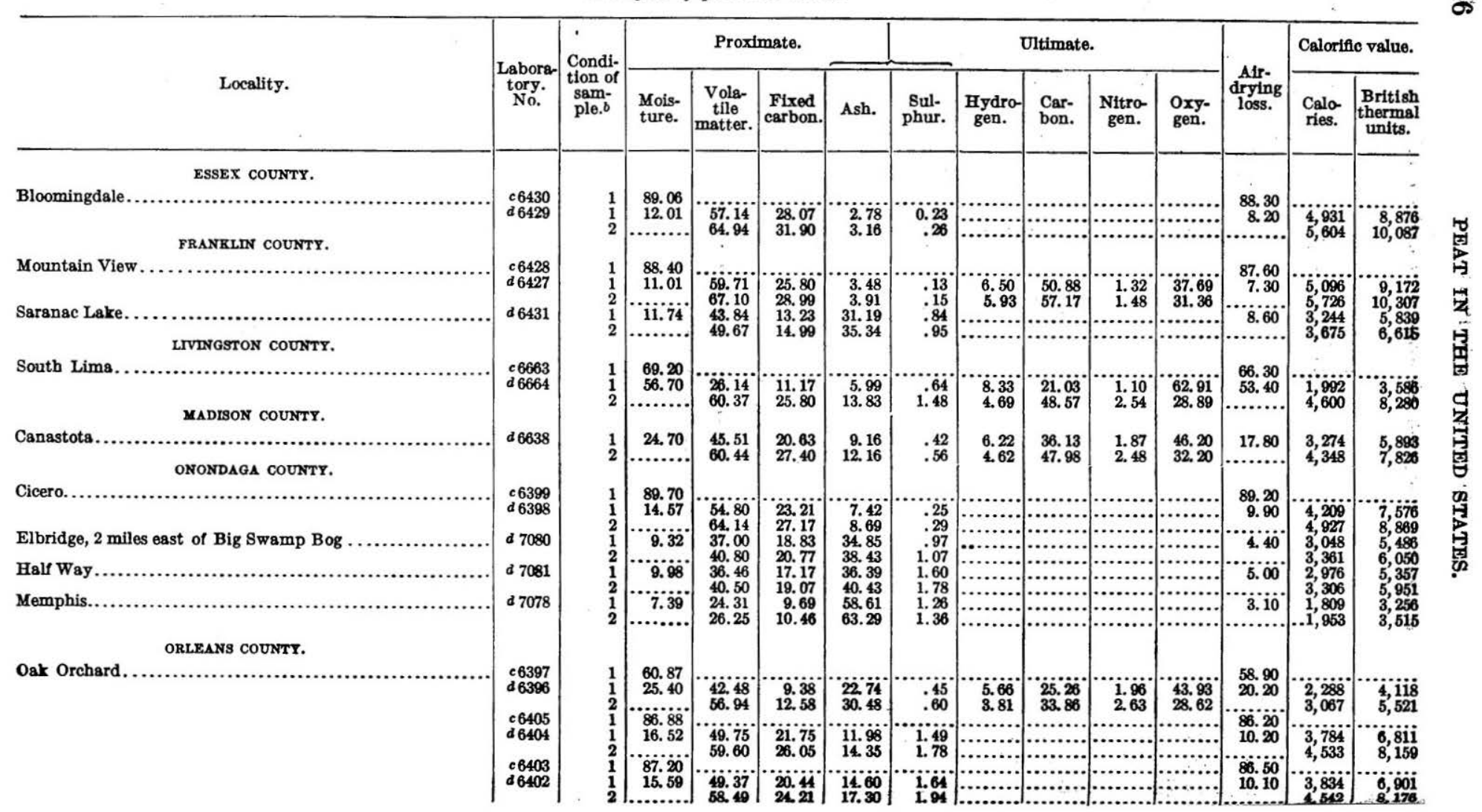


OSWEGO COUNTY.

Fulton, 7 miles east of

Hastings, 4 miles west of Central Square.

Palermo.

West Monroe.

ST. LAWRENCE COUNTY.

Ogdensburg, near Black Lake Bog (machine peat)

Ogdensburg, near Black Lake Bog (peat from barge)

Ogdensburg, near Black Lake Bog (machine peat) .

Ogdensburg, near Black Lake Bog (briquetted peat)

STEUBEN COUNTY.

Arkport.

$c 7083$
$d 7082$

\begin{tabular}{|c|c|c|c|c|c|c|c|c|c|c|c|c|c|}
\hline c6636 & 1 & 93. 96 & & & & & & & & & 93.50 & \multirow{4}{*}{$\begin{array}{l}2,280 \\
5,029 \\
2,557 \\
2,750\end{array}$} & \multirow{4}{*}{$\begin{array}{l}4,100 \\
9,052 \\
4,603 \\
4,950\end{array}$} \\
\hline d 6637 & $\frac{1}{2}$ & 54.66 & 29.15 & 12.44 & 3. 75 & .17 & & …… & ……… & ......... & $\begin{array}{c}51.60 \\
\cdots \cdots \cdots\end{array}$ & & \\
\hline d 7079 & 1 & 7.03 & 31.60 & 17.46 & 43. 91 & .74 & $\ldots . . . .$. & ….......... & …….. & ; & \multirow[t]{2}{*}{2.80} & & \\
\hline c6634 & 2 & 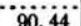 & & & & & $\cdots \cdots$ & & & & & & \\
\hline d 6635 & 1 & 74.78 & 15. 29 & 7.47 & 2.46 & - 16 & & & & & $\begin{array}{l}89.40 \\
72.40\end{array}$ & 1,237 & \multirow{2}{*}{$\begin{array}{l}2,297 \\
8,829\end{array}$} \\
\hline $\begin{array}{l}c 6401 \\
d 6400\end{array}$ & 1 & 92.27 & & & & & & & & & \multirow{2}{*}{$\begin{array}{l}91.90 \\
12.10\end{array}$} & & \\
\hline & $\frac{1}{2}$ & $\begin{array}{r}18.45 \\
.\end{array}$ & $\begin{array}{l}44.55 \\
54.63\end{array}$ & $\begin{array}{l}24.55 \\
30.10\end{array}$ & $\begin{array}{l}12.45 \\
15.27\end{array}$ & $\begin{array}{r}.75 \\
.92\end{array}$ & $\begin{array}{l}5.95 \\
4.78\end{array}$ & $\begin{array}{l}41.63 \\
51.05\end{array}$ & $\begin{array}{l}1.71 \\
2.10\end{array}$ & $\begin{array}{l}37.51 \\
25.88\end{array}$ & & $\begin{array}{l}3,994 \\
4,897\end{array}$ & $\begin{array}{r}7,189 \\
8,815\end{array}$ \\
\hline $\begin{array}{l}c 6393 \\
d 6392\end{array}$ & 1 & 11.54 & & & & & & & & & \multirow{2}{*}{$\begin{array}{l}6.20 \\
5.00\end{array}$} & & \multirow{7}{*}{$\begin{array}{r}7,015 \\
7,971 \\
6,853 \\
8,237 \\
6,709 \\
8,050 \\
6,860 \\
8,203\end{array}$} \\
\hline$d 6392$ & $\frac{1}{2}$ & 12.00 & $\begin{array}{l}49.43 \\
56.17\end{array}$ & $\begin{array}{r}21.59 \\
24.53\end{array}$ & $\begin{array}{l}16.98 \\
19.30\end{array}$ & $\begin{array}{r}.91 \\
.03\end{array}$ & $\cdots$ & $\cdots \cdots \cdot$ & …..... & & & $\begin{array}{l}3,897 \\
4428\end{array}$ & \\
\hline d 6436 & $\frac{1}{2}$ & 16.81 & 46.39 & 21.67 & 15.13 & .90 & . & ; & ; & $\cdots . . .$. & 8.90 & 3,807 & \\
\hline d6394 & 1 & 16.67 & 45.81 & 21.47 & $\begin{array}{l}16.05 \\
16.18\end{array}$ & $\begin{array}{l}.00 \\
.82\end{array}$ & .... & $\cdots$ & & & \multirow{2}{*}{8.10} & 3.727 & \\
\hline & 2 & & 54. 97 & 25.77 & 19. 26 & .98 & & & & & & 4,472 & \\
\hline d 6395 & 1 & 16. 37 & 47.44 & 20.40 & 15. 79 & 1.06 & .... & $\cdots$ & & & 9.10 & 3,811 & \\
\hline & 2 & ......... & 56.73 & 24.39 & 18.88 & 1.27 & & & & & & 4,557 & \\
\hline $\begin{array}{l}\text { c 7083 } \\
\text { d 7082 }\end{array}$ & 1 & 87.45 & & & & & & & & & \multirow{3}{*}{$\begin{array}{r}86.60 \\
7.00 \\
\ldots \ldots . .\end{array}$} & & \\
\hline d 7082 & 1 & 13. 18 & 43. 33 & 20.50 & 22.99 & 1. 38 & ...... & ...... & & & & 3,493 & 6,287 \\
\hline & & & & & & & & & & & & & \\
\hline
\end{tabular}

a Davis, C. A., The uses of peat for fuel and other purposes: Bur. Mines Bull. 16 .pp. 198-199, 1911. b 1, Native peat; 2, Moisture-iree peat. 
OFIO.

Analyses of moisture-free peat and muck."

[Bureau of Mines.]

\begin{tabular}{|c|c|c|c|c|c|c|c|c|c|c|}
\hline County. & $\begin{array}{c}\text { De- } \\
\text { posit } \\
\text { No. }\end{array}$ & $\begin{array}{l}\text { Labora- } \\
\text { tory No. }\end{array}$ & $\begin{array}{l}\text { Mois- } \\
\text { ture. }\end{array}$ & $\begin{array}{l}\text { Volatile } \\
\text { matter. }\end{array}$ & $\begin{array}{c}\text { Fixed } \\
\text { carbon. }\end{array}$ & Ash. & & $\begin{array}{l}\text { Sul- } \\
\text { phur. }\end{array}$ & Iron. & $\begin{array}{l}\text { Calori- } \\
\text { flo } \\
\text { value } \\
\text { (British } \\
\text { thermsl } \\
\text { units). }\end{array}$ \\
\hline 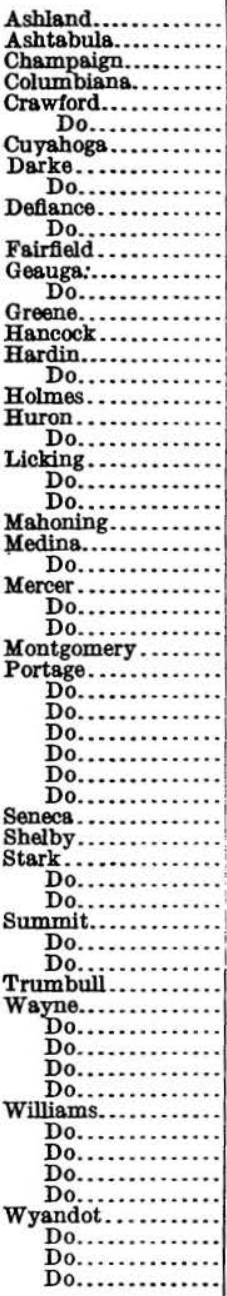 & \begin{tabular}{|r|}
1 \\
2 \\
3 \\
4 \\
5 \\
6 \\
7 \\
8 \\
9 \\
10 \\
11 \\
12 \\
13 \\
14 \\
15 \\
16 \\
17 \\
18 \\
19 \\
20 \\
21 \\
22 \\
23 \\
24 \\
25 \\
26 \\
27 \\
28 \\
29 \\
30 \\
31 \\
32 \\
33 \\
34 \\
35 \\
36 \\
37 \\
38 \\
39 \\
40 \\
41 \\
42 \\
43 \\
44 \\
45 \\
46 \\
47 \\
48 \\
49 \\
50 \\
51 \\
52 \\
53 \\
54 \\
55 \\
56 \\
58 \\
61 \\
\end{tabular} & \begin{tabular}{|l}
$73-11122$ \\
$44-10773$ \\
$60-11008$ \\
$24-$ \\
$64-11014$ \\
$89-12477$ \\
$49-10778$ \\
$88-12476$ \\
$87-12475$ \\
$82-11348$ \\
$83-11349$ \\
$66-11115$ \\
$49-10778$ \\
$51-10798$ \\
$85-12473$ \\
$38-10687$ \\
$61-11010$ \\
$62-11012$ \\
$72-11121$ \\
$42-10733$ \\
$64-11014$ \\
$43-10772$ \\
$40-10709$ \\
$41-10710$ \\
$50-10797$ \\
$70-11119$ \\
$71-11120$ \\
$81-11347$ \\
$80-11321$ \\
$79-11320$ \\
$84-12472$ \\
$55-10814$ \\
$52-10806$ \\
$53-10805$ \\
$56-10815$ \\
$47-10777$ \\
$46-11007$ \\
$54-10813$ \\
$38-10687$ \\
$86-12474$ \\
$74-11147$ \\
$75-11148$ \\
$76-11149$ \\
$59-10832$ \\
$58-10830$ \\
$57-10829$ \\
$45-10774$ \\
$67-11116$ \\
$68-11117$ \\
$69-11118$ \\
$77-11318$ \\
$78-11319$ \\
$33-13180$ \\
$34-13181$ \\
$63-11046$ \\
$90-13182$ \\
$65-11047$ \\
$35-10684$ \\
$36-10685$ \\
$37-10686$ \\
$39-10688$
\end{tabular} & $\begin{array}{r}7.14 \\
9.08 \\
10.73 \\
10.96 \\
9.24 \\
13.53 \\
9.01 \\
12.72 \\
16.24 \\
8.58 \\
8.97 \\
7.58 \\
9.01 \\
10.11 \\
15.56 \\
11.02 \\
10.85 \\
10.81 \\
6.96 \\
8.77 \\
13.53 \\
9.90 \\
7.87 \\
9.23 \\
10.01 \\
7.53 \\
7.67 \\
8.72 \\
9.28 \\
10.23 \\
7.02 \\
8.65 \\
11.25 \\
8.41 \\
9.36 \\
10.40 \\
9.12 \\
8.98 \\
11.02 \\
16.28 \\
10.43 \\
8.82 \\
8.01 \\
11.35 \\
10.54 \\
8.91 \\
8.38 \\
7.00 \\
10.35 \\
9.85 \\
8.84 \\
7.44 \\
7.60 \\
7.27 \\
6.99 \\
10.12 \\
10.41 \\
9.09 \\
8.38 \\
12 \\
\end{array}$ & 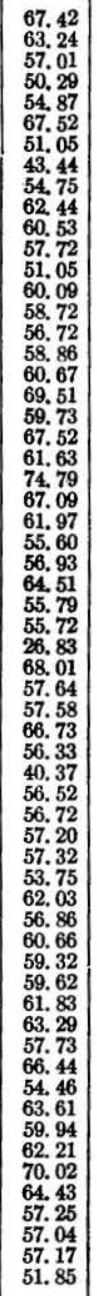 & 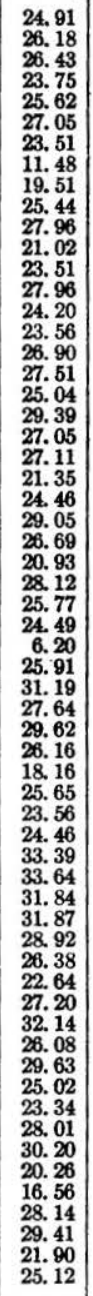 & 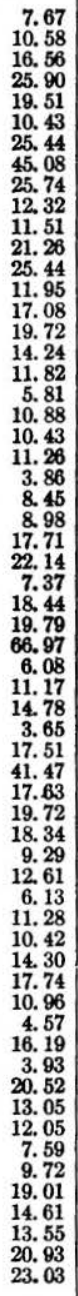 & $\begin{array}{l}2.39 \\
2.45 \\
2.56 \\
2.42 \\
2.70 \\
3.23 \\
2.13 \\
2.30 \\
3.05 \\
2.58 \\
2.90 \\
3.25 \\
2.13 \\
2.24 \\
3.60 \\
3.48 \\
3.37 \\
3.41 \\
2.38 \\
2.84 \\
3.23 \\
2.21 \\
2.52 \\
1.01 \\
2.66 \\
2.58 \\
2.55 \\
2.77 \\
3.19 \\
2.97 \\
.93 \\
3.01 \\
2.33 \\
1.62 \\
1.66 \\
2.46 \\
1.72 \\
2.21 \\
3.48 \\
2.66 \\
2.94 \\
2.79 \\
3.39 \\
2.72 \\
2.88 \\
2.34 \\
2.73 \\
2.97 \\
2.22 \\
2.59 \\
2.30 \\
2.41 \\
2.63 \\
2.93 \\
2.03 \\
3.36 \\
2.43 \\
2.98 \\
2.84 \\
2.92 \\
2.16\end{array}$ & 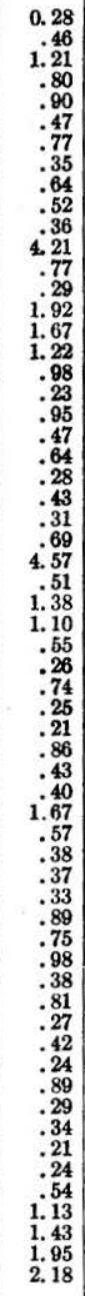 & 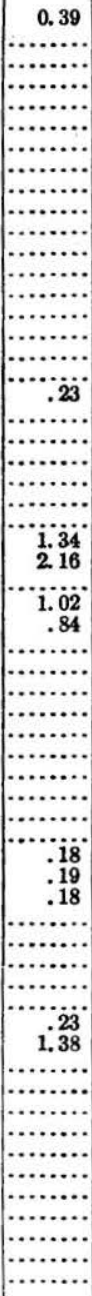 & $\begin{array}{l}9,070 \\
8,966 \\
7,567 \\
7,520 \\
7,448 \\
8,683 \\
7,504 \\
4,637 \\
6,797 \\
8,420 \\
8,566 \\
7,603 \\
7,504 \\
8,741 \\
8,248 \\
7,749 \\
7,916 \\
7,996 \\
9,571 \\
8,914 \\
8,683 \\
8,122 \\
8,338 \\
8,626 \\
8,737 \\
8,168 \\
7,132 \\
8,915 \\
7,781 \\
7,763 \\
2,914 \\
9,443 \\
8,564 \\
8,996 \\
9,736 \\
8,190 \\
5,121 \\
8,402 \\
7,749 \\
7,675 \\
7,794 \\
8,498 \\
8,842 \\
8,861 \\
8,703 \\
8,372 \\
8,147 \\
8,608 \\
9,569 \\
8,280 \\
9,148 \\
7,875 \\
8,345 \\
8,532 \\
9,389 \\
8,914 \\
8,905 \\
8,378 \\
8,482 \\
7,682 \\
7,666\end{array}$ \\
\hline
\end{tabular}

a Dachnowskd, Alfred, Peat deposits of Ohio, their origin, formation, and uses: Ohio Geol. Survey Bull. 16, 4th ser., pp.366-367, 1912 . 
PENNBYIVANIA.

Analyses of moisture-free peat.

[H. M. Cooper, Bureau of Mines, analyst.]

\begin{tabular}{|c|c|c|c|c|c|c|c|c|c|}
\hline \multirow[b]{2}{*}{ County. } & \multirow{2}{*}{$\begin{array}{c}\text { Local- } \\
\text { ity } \\
\text { No. }\end{array}$} & \multirow[b]{2}{*}{$\begin{array}{l}\text { Analy- } \\
\text { sis No. }\end{array}$} & \multicolumn{3}{|c|}{ Proximate. } & \multicolumn{2}{|c|}{ Ultimate. } & \multicolumn{2}{|c|}{ Calorifle value. } \\
\hline & & & $\begin{array}{c}\text { Vola- } \\
\text { tile } \\
\text { matter. }\end{array}$ & $\begin{array}{l}\text { Fixed } \\
\text { carbon. }\end{array}$ & Ash. & $\begin{array}{l}\text { Nitro- } \\
\text { gen. }\end{array}$ & $\begin{array}{l}\text { Sul- } \\
\text { phur. }\end{array}$ & $\begin{array}{l}\text { Calo- } \\
\text { ries. }\end{array}$ & $\begin{array}{l}\text { British } \\
\text { thermal } \\
\text { units. }\end{array}$ \\
\hline 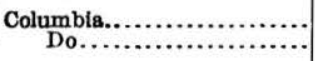 & $\begin{array}{l}1 \\
2\end{array}$ & $\begin{array}{l}1 \\
2\end{array}$ & $\begin{array}{l}45.84 \\
52.92\end{array}$ & $\begin{array}{l}28.99 \\
19.29\end{array}$ & $\begin{array}{l}25.17 \\
27.79\end{array}$ & $\begin{array}{l}2.03 \\
2.07\end{array}$ & $\begin{array}{l}0.61 \\
1.49\end{array}$ & $\begin{array}{l}4,257 \\
4,041\end{array}$ & $\begin{array}{l}7,662 \\
7,274\end{array}$ \\
\hline
\end{tabular}

RHODE ISLAND.

Analyses of moisture-free peat and muck.

[H. M. Cooper, Bureau of Mines, analyst.]

\begin{tabular}{|c|c|c|c|c|c|c|c|c|c|c|}
\hline \multirow{2}{*}{$\begin{array}{l}\text { Local- } \\
\text { ity } \\
\text { No. }\end{array}$} & \multirow[b]{2}{*}{ County. } & \multirow{2}{*}{$\begin{array}{c}\text { Ansly- } \\
\text { sis and } \\
\text { sample } \\
\text { No. }\end{array}$} & \multicolumn{3}{|c|}{ Proximate. } & \multicolumn{3}{|c|}{ Ultimate. } & \multicolumn{2}{|c|}{ Calorific value. } \\
\hline & & & $\begin{array}{c}\text { Vola- } \\
\text { tile } \\
\text { matter. }\end{array}$ & $\begin{array}{c}\text { Fixed } \\
\text { carbon. }\end{array}$ & Ash. & $\begin{array}{l}\text { Sul- } \\
\text { phur. }\end{array}$ & $\begin{array}{c}\text { Nitro- } \\
\text { gen. }\end{array}$ & $\begin{array}{l}\text { Oxy- } \\
\text { gen. }\end{array}$ & $\begin{array}{l}\text { Calo- } \\
\text { ries. }\end{array}$ & $\begin{array}{l}\text { British } \\
\text { thermal } \\
\text { units. }\end{array}$ \\
\hline $\begin{array}{l}1 \\
2 \\
4 \\
5\end{array}$ & 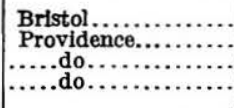 & $\begin{array}{l}52 \\
49 \\
51 \\
50\end{array}$ & $\begin{array}{l}42.43 \\
45.19 \\
40.65 \\
44.49\end{array}$ & $\begin{array}{l}19.82 \\
31.11 \\
21.85 \\
34.64\end{array}$ & $\begin{array}{l}37.75 \\
23.70 \\
37.50 \\
20.87\end{array}$ & $\begin{array}{r}1.01 \\
.30 \\
.54 \\
.44\end{array}$ & $\begin{array}{l}1.51 \\
1.56 \\
1.59 \\
1.77\end{array}$ & $\begin{array}{l}\cdots \\
\cdots \cdots \\
\cdots \cdots \\
\cdots\end{array}$ & $\begin{array}{r}\ddot{4}, 298 \\
\hdashline, 612\end{array}$ & $\begin{array}{r}7,737 \\
8,301\end{array}$ \\
\hline
\end{tabular}

VERMONT.

Analyses of moisture-free peat and muck.

[H. M. Cooper, Bureau of Mines, anslyst.]

\begin{tabular}{|c|c|c|c|c|c|c|c|c|c|c|c|c|}
\hline \multirow{2}{*}{$\begin{array}{c}\text { Lo- } \\
\text { cali- } \\
\text { ty } \\
\text { No. }\end{array}$} & \multirow[b]{2}{*}{ County. } & \multirow{2}{*}{$\begin{array}{c}\text { Sam- } \\
\text { ple } \\
\text { and } \\
\text { analy- } \\
\text { sis } \\
\text { No. }\end{array}$} & \multicolumn{3}{|c|}{ Proximste. } & \multicolumn{5}{|c|}{ Ultimate. } & \multicolumn{2}{|c|}{ Calorific value. } \\
\hline & & & $\begin{array}{l}\text { Vola- } \\
\text { tille } \\
\text { mat- } \\
\text { ter. }\end{array}$ & $\begin{array}{c}\text { Fixed } \\
\text { car- } \\
\text { bon. }\end{array}$ & Ash. & $\begin{array}{l}\text { Sul- } \\
\text { phur. }\end{array}$ & $\begin{array}{l}\text { Hy- } \\
\text { dro- } \\
\text { gen. }\end{array}$ & $\begin{array}{l}\text { Car- } \\
\text { bon. }\end{array}$ & $\begin{array}{l}\text { Nitro- } \\
\text { gen. }\end{array}$ & $\begin{array}{l}\text { Oxy- } \\
\text { gen. }\end{array}$ & $\begin{array}{l}\text { Calo- } \\
\text { ries. }\end{array}$ & $\begin{array}{c}\text { British } \\
\text { ther- } \\
\text { mal } \\
\text { units. }\end{array}$ \\
\hline $\begin{array}{r}1 \\
1 \\
2 \\
3 \\
3 \\
4 \\
8 \\
9 \\
14 \\
16 \\
19\end{array}$ & 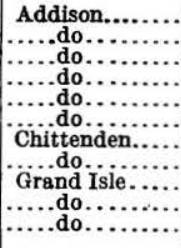 & $\begin{array}{l}9 \\
9 \mathrm{a} \\
10 \\
11 \\
11 \mathrm{a} \\
12 \\
13 \\
14 \\
15 \\
16 \\
17\end{array}$ & $\begin{array}{l}40.06 \\
48.70 \\
37.37 \\
56.39 \\
38.75 \\
48.71 \\
42.01 \\
25.26 \\
34.47 \\
48.70 \\
36.01\end{array}$ & $\begin{array}{l}42.08 \\
10.07 \\
14.02 \\
35.53 \\
21.05 \\
40.25 \\
45.02 \\
37.36 \\
21.38 \\
45.19 \\
52.53\end{array}$ & $\begin{array}{r}17.86 \\
41.23 \\
48.61 \\
8.28 \\
40.20 \\
11.04 \\
12.97 \\
37.38 \\
44.15 \\
6.11 \\
11.46\end{array}$ & $\begin{array}{r}0.75 \\
.56 \\
1.19 \\
.56 \\
.65 \\
.57 \\
.39 \\
.43 \\
.22 \\
.28 \\
1.04\end{array}$ & $\begin{array}{r} \\
\cdots \\
\cdots \\
\cdots \\
\cdots\end{array}$ & 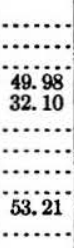 & $\begin{array}{l}2.37 \\
1.68 \\
1.81 \\
2.65 \\
2.24 \\
2.73 \\
2.18 \\
2.78 \\
1.68 \\
1.90 \\
2.44\end{array}$ & 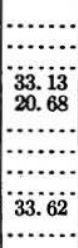 & \begin{tabular}{r}
4,318 \\
$\ldots \ldots \ldots$ \\
$4,77 i$ \\
\hdashline 4,689 \\
4,704 \\
$\ldots \ldots \ldots$ \\
\hdashline, 944 \\
4,781
\end{tabular} & 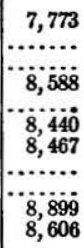 \\
\hline
\end{tabular}


Analyses showing average composition of moisture-free peat.

\begin{tabular}{|c|c|c|c|c|c|c|c|c|c|}
\hline \multirow[b]{2}{*}{ * } & \multirow{2}{*}{$\begin{array}{l}\text { No. of } \\
\text { samples } \\
\text { repre- } \\
\text { sented. }\end{array}$} & \multicolumn{2}{|c|}{ Proximate. } & \multicolumn{4}{|c|}{ Ultimate. } & \multicolumn{2}{|c|}{ Calorific value. } \\
\hline & & $\begin{array}{c}\text { Volatile } \\
\text { mat- } \\
\text { ter. }\end{array}$ & Ash. & $\begin{array}{c}\text { Hydro- } \\
\text { gen. }\end{array}$ & Carbon. & $\begin{array}{l}\text { Nitro- } \\
\text { gen. }\end{array}$ & $\begin{array}{c}\text { Oxygen } \\
\text { and } \\
\text { sul- } \\
\text { phur. }\end{array}$ & $\begin{array}{l}\text { Calo- } \\
\text { ries. }\end{array}$ & $\begin{array}{c}\text { British } \\
\text { thermal } \\
\text { units. }\end{array}$ \\
\hline 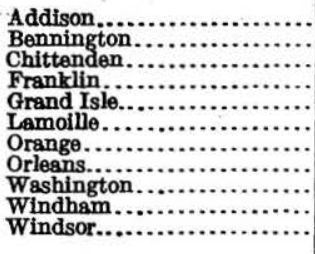 & $\begin{array}{r}14 \\
13 \\
24 \\
15 \\
36 \\
8 \\
5 \\
5 \\
3 \\
6 \\
9\end{array}$ & $\begin{array}{l}88.22 \\
86.50 \\
92.96 \\
91.97 \\
88.22 \\
90.03 \\
84.84 \\
90.48 \\
92.17 \\
92.26 \\
94.75\end{array}$ & $\begin{array}{r}11.78 \\
13.50 \\
7.04 \\
8.03 \\
11.78 \\
9.97 \\
15.16 \\
9.52 \\
7.83 \\
6.74 \\
5.25\end{array}$ & $\begin{array}{l}4.30 \\
4.36 \\
4.87 \\
4.52 \\
4.65 \\
4.09 \\
4.41 \\
4.42 \\
5.24 \\
4.92 \\
5.06\end{array}$ & $\begin{array}{l}47.91 \\
46.78 \\
52.04 \\
50.23 \\
45.87 \\
48.83 \\
45.56 \\
49.82 \\
49.84 \\
50.32 \\
52.39\end{array}$ & $\begin{array}{l}2.62 \\
2.29 \\
1.85 \\
1.89 \\
2.70 \\
2.13 \\
2.78 \\
2.33 \\
2.70 \\
1.88 \\
2.07\end{array}$ & $\begin{array}{l}33.39 \\
33.07 \\
34.20 \\
35.33 \\
35.00 \\
34.98 \\
32.09 \\
33.91 \\
34.69 \\
36.14 \\
35.23\end{array}$ & $\begin{array}{l}4,302 \\
4,248 \\
4,832 \\
4,530 \\
4,206 \\
4,272 \\
4,183 \\
4,493 \\
4,743 \\
4,651 \\
4,891 \\
\end{array}$ & $\begin{array}{l}7,740 \\
7,630 \\
8,690 \\
8,18 \\
7,580 \\
7,690 \\
7,520 \\
8,090 \\
8,520 \\
8,360 \\
8,800\end{array}$ \\
\hline General average....... & $\ldots \ldots \ldots$ & 90.09 & 9.91 & 4.61 & 48.71 & 2.29 & 34.48 & 4,452 & 8,073 \\
\hline
\end{tabular}

a Vermant Agr. Exper. Sta. Bull. 165, p. 219, 1912.

\section{VIRGINIA AND NORTH CAROIINA.}

Analyses of peat and muck.

[H. M. Cooper, Bureau of Mines, analyst.]

\begin{tabular}{|c|c|c|c|c|c|c|c|c|c|c|c|}
\hline \multirow{2}{*}{ Locality. } & \multirow{2}{*}{$\begin{array}{l}\text { Sam- } \\
\text { ple } \\
\text { No. }\end{array}$} & \multirow{2}{*}{ Hole. } & \multirow{2}{*}{$\begin{array}{c}\text { Condi- } \\
\text { tion } \\
\text { of ssm- } \\
\text { ple.a }\end{array}$} & \multicolumn{3}{|c|}{ Proximate. } & \multicolumn{3}{|c|}{ Ultimate. } & \multicolumn{2}{|c|}{ Calorifle value. } \\
\hline & & & & $\begin{array}{l}\text { Mois- } \\
\text { ture. }\end{array}$ & $\begin{array}{c}\text { Volstile } \\
\text { matter. }\end{array}$ & $\begin{array}{l}\text { Fixed } \\
\text { carbon. }\end{array}$ & Ash. & $\begin{array}{l}\text { Sul- } \\
\text { phur. }\end{array}$ & $\begin{array}{l}\text { Nitro- } \\
\text { gen. }\end{array}$ & $\begin{array}{l}\text { Calo- } \\
\text { ries. }\end{array}$ & $\begin{array}{c}\text { British } \\
\text { thermal } \\
\text { units. }\end{array}$ \\
\hline VIRGINIA. & & & & & & & & & & & \\
\hline Norfolk County..... & 1 & A & 1 & 14.01 & $\begin{array}{l}45.42 \\
52.82\end{array}$ & $\begin{array}{l}33.92 \\
39.46\end{array}$ & $\begin{array}{l}6.64 \\
7.72\end{array}$ & 0.31 & 1.73 & $\begin{array}{l}4,690 \\
5,454\end{array}$ & $\begin{array}{l}8,442 \\
9,818\end{array}$ \\
\hline Do.... & 2 & $\mathbf{A}$ & $\begin{array}{l}1 \\
2\end{array}$ & $\begin{array}{l}32.93 \\
\ldots \ldots \ldots\end{array}$ & $\begin{array}{l}42.42 \\
63.25\end{array}$ & $\begin{array}{l}19.62 \\
29.25\end{array}$ & $\begin{array}{l}5.03 \\
7.50\end{array}$ & .23 & $\begin{array}{l}1.38 \\
2.06\end{array}$ & $\begin{array}{l}3,655 \\
5,449\end{array}$ & $\begin{array}{l}6,578 \\
9,808\end{array}$ \\
\hline Do.... & 3 & B & $\begin{array}{l}1 \\
2\end{array}$ & 17.93 & $\begin{array}{l}46.57 \\
56.74\end{array}$ & $\begin{array}{l}20.15 \\
24.56\end{array}$ & $\begin{array}{l}15.35 \\
18.70\end{array}$ & .19 & $\begin{array}{l}1.31 \\
1.60\end{array}$ & $\begin{array}{l}4,052 \\
4,937\end{array}$ & $\begin{array}{l}7,293 \\
8.898\end{array}$ \\
\hline Do..... & 4 & B & 1 & 18.88 & 47. 55 & 18. 49 & 15. 08 & .19 & 1.27 & 4,021 & 7,238 \\
\hline Do... & 5 & D & 1 & 9.38 & $\begin{array}{l}48.05 \\
53.02\end{array}$ & 18.72 & $\begin{array}{l}\text { 23. } 85 \\
23.32\end{array}$ & .28 & $\begin{array}{l}1.67 \\
1.84\end{array}$ & $\begin{array}{l}3,881 \\
4,282\end{array}$ & 6,985 \\
\hline $\begin{array}{l}\text { Nansemond County. } \\
\text { NORTH CAROLINA. }\end{array}$ & 6 & $\mathbf{F}$ & $\begin{array}{l}\tilde{1} \\
2\end{array}$ & 6.43 & $\begin{array}{l}30.32 \\
32.40\end{array}$ & $\begin{array}{l}10.92 \\
11.67\end{array}$ & $\begin{array}{l}52.33 \\
55.93\end{array}$ & .25 & $\begin{array}{l}1.07 \\
1.14\end{array}$ & ........... & ……..... \\
\hline Currituck County... & 7 & $\mathbf{J}$ & $\begin{array}{l}1 \\
2\end{array}$ & $\begin{array}{r}8.23 \\
\cdots . . . .\end{array}$ & $\begin{array}{l}52.05 \\
56.72\end{array}$ & $\begin{array}{l}33.54 \\
36.55\end{array}$ & $\begin{array}{l}6.18 \\
6.73\end{array}$ & .26 & $\begin{array}{l}1.60 \\
1.74\end{array}$ & $\begin{array}{l}5,163 \\
5,628\end{array}$ & $\begin{array}{r}9,294 \\
10,127\end{array}$ \\
\hline
\end{tabular}

a 1, As received in the laboratory. 2, Moisture free.

Analyses of peat from Pasquotank County, N. C. ${ }^{a}$

[F. M. Stanton, U. S. Geol. Survey, analyst.]

\begin{tabular}{|c|c|c|c|c|c|c|c|c|c|c|c|c|c|}
\hline \multirow{2}{*}{$\begin{array}{l}\text { Lab- } \\
\text { ora- } \\
\text { tory } \\
\text { No. }\end{array}$} & \multirow{2}{*}{$\begin{array}{c}\text { Condi- } \\
\text { tion } \\
\text { of } \\
\text { sam- } \\
\text { ple.b }\end{array}$} & \multicolumn{4}{|c|}{ Proximate. } & \multicolumn{5}{|c|}{ Ultimate. } & \multirow{2}{*}{$\begin{array}{l}\text { Air- } \\
\text { drying } \\
\text { loss. }\end{array}$} & \multicolumn{2}{|c|}{ Calorific value. } \\
\hline & & $\begin{array}{l}\text { Mois- } \\
\text { ture. }\end{array}$ & $\begin{array}{l}\text { Vola- } \\
\text { tile } \\
\text { mat- } \\
\text { ter. }\end{array}$ & $\begin{array}{c}\text { Fixed } \\
\text { car- } \\
\text { bon. }\end{array}$ & Ash. & $\begin{array}{c}\text { Sul- } \\
\text { phur. }\end{array}$ & $\begin{array}{c}\text { Hydro- } \\
\text { gen. }\end{array}$ & $\begin{array}{l}\text { Car- } \\
\text { bon. }\end{array}$ & $\begin{array}{l}\text { Nitro- } \\
\text { gen. }\end{array}$ & $\begin{array}{l}\text { Oxy- } \\
\text { gen. }\end{array}$ & & $\begin{array}{l}\text { Calo- } \\
\text { ries. }\end{array}$ & $\begin{array}{c}\text { British } \\
\text { thermal } \\
\text { units. }\end{array}$ \\
\hline $\begin{array}{c}\text { c } 5585 \\
\ldots \ldots\end{array}$ & $\begin{array}{l}1 \\
2\end{array}$ & $\begin{array}{r}85.12 \\
\ldots \ldots\end{array}$ & $\begin{array}{r}7.72 \\
51.88\end{array}$ & $\begin{array}{r}4.29 \\
28.83\end{array}$ & $\begin{array}{r}2.87 \\
19.29\end{array}$ & $\begin{array}{l}0.33 \\
2.22\end{array}$ & $\begin{array}{r}10.06 \\
4.03\end{array}$ & $\begin{array}{r}7.06 \\
47.45\end{array}$ & $\begin{array}{l}0.25 \\
1.68\end{array}$ & $\begin{array}{l}79.43 \\
25.33\end{array}$ & $\begin{array}{c}83.20 \\
\ldots \ldots\end{array}$ & $\begin{array}{r}682 \\
4,583\end{array}$ & $\begin{array}{l}1,228 \\
8,249\end{array}$ \\
\hline
\end{tabular}

a Davis, C. A., The uses of peat for fuel and other purposes: Bur. Mines Bull. 16, p. 199, 1911.

$b$ 1, Native peat. 2, Moisture-free peat. 
PROPERTIES OF PEAT.

wrsoorsin.

Analyses of peat and muck. ${ }^{a}$

[Survey of 1903.]

\begin{tabular}{|c|c|c|c|c|c|c|c|c|c|c|}
\hline \multirow{2}{*}{$\begin{array}{l}\text { Location (nearest } \\
\text { town or city). }\end{array}$} & \multirow{2}{*}{$\begin{array}{l}\text { Deposit } \\
\text { No. }\end{array}$} & \multirow{2}{*}{$\begin{array}{c}\text { Sample } \\
\text { No. }\end{array}$} & \multirow{2}{*}{$\begin{array}{c}\begin{array}{c}\text { As re- } \\
\text { ceived. }\end{array} \\
\begin{array}{l}\text { Mois- } \\
\text { ture. }\end{array}\end{array}$} & \multicolumn{4}{|c|}{ Moisture-free. } & \multicolumn{3}{|c|}{ Combustible contents. } \\
\hline & & & & $\begin{array}{l}\text { Volatile } \\
\text { matter. }\end{array}$ & $\begin{array}{l}\text { Fixed } \\
\text { carbon. }\end{array}$ & Ash. & $\begin{array}{c}\text { British } \\
\text { thermsl } \\
\text { units. }\end{array}$ & $\begin{array}{l}\text { Volatile } \\
\text { matter. }\end{array}$ & $\begin{array}{l}\text { Fixed } \\
\text { carbon. }\end{array}$ & $\begin{array}{c}\text { British } \\
\text { thermal } \\
\text { units. }\end{array}$ \\
\hline $\begin{array}{r}\text { Stoughton. } \\
\text { Do.... } \\
\text { Do.... } \\
\text { Do.... } \\
\text { Do.... } \\
\text { Whitewste } \\
\text { Do..... } \\
\text { Do.... } \\
\text { Do.... } \\
\text { Do.... } \\
\text { Do.... } \\
\text { Do..... } \\
\text { Do..... } \\
\text { Do..... } \\
\text { Do.... } \\
\text { Lake Beula } \\
\text { Do..... } \\
\text { Do.... }\end{array}$ & $\begin{array}{l}1 \\
1 \\
1 \\
1 \\
1 \\
2 \\
2 \\
2 \\
2 \\
2 \\
3 \\
3 \\
3 \\
4 \\
4 \\
5 \\
5 \\
5\end{array}$ & $\begin{array}{l}1 \\
2 \\
3 \\
4 \\
5 \\
1 \\
2 \\
3 \\
4 \\
5 \\
1 \\
2 \\
3 \\
1 \\
2 \\
1 \\
2 \\
3\end{array}$ & $\begin{array}{r}69.7 \\
65.2 \\
87.0 \\
74.4 \\
85.3 \\
69.4 \\
85.5 \\
82.8 \\
b 33.4 \\
b 14.0 \\
83.0 \\
82.9 \\
80.7 \\
81.1 \\
80.7 \\
b 58.5 \\
b 14.9 \\
83.5\end{array}$ & $\begin{array}{l}63.3 \\
63.1 \\
62.4 \\
59.3 \\
63.3 \\
38.4 \\
60.4 \\
58.2 \\
57.4 \\
59.7 \\
52.2 \\
62.0 \\
57.2 \\
55.7 \\
53.1 \\
58.5 \\
56.7 \\
59.3\end{array}$ & $\begin{array}{l}25.3 \\
24.5 \\
26.5 \\
20.5 \\
23.6 \\
16.9 \\
27.8 \\
26.6 \\
25.4 \\
29.4 \\
28.3 \\
28.7 \\
27.0 \\
25.1 \\
32.3 \\
28.6 \\
30.9 \\
27.5\end{array}$ & $\begin{array}{r}11.4 \\
12.4 \\
11.1 \\
20.2 \\
13.1 \\
44.7 \\
11.8 \\
15.2 \\
17.2 \\
10.9 \\
19.5 \\
9.3 \\
15.8 \\
19.2 \\
14.6 \\
12.9 \\
12.4 \\
13.2\end{array}$ & $\begin{array}{l}9,320 \\
8,980 \\
8,929 \\
6,190 \\
8,570 \\
6,040 \\
9,440 \\
9,040 \\
9,370 \\
8,600 \\
8,060 \\
8,370 \\
8,600 \\
7,920 \\
8,490 \\
8,280 \\
8,870 \\
8,870\end{array}$ & $\begin{array}{l}71.5 \\
72.0 \\
70.4 \\
74.4 \\
73.9 \\
69.5 \\
68.5 \\
68.7 \\
69.4 \\
67.0 \\
64.9 \\
68.4 \\
68.0 \\
69.0 \\
62.3 \\
67.3 \\
64.7 \\
68.1\end{array}$ & $\begin{array}{l}28.5 \\
28.0 \\
29.8 \\
25.7 \\
26.1 \\
30.6 \\
31.6 \\
31.4 \\
30.7 \\
33.0 \\
35.2 \\
31.6 \\
32.0 \\
31.1 \\
37.8 \\
32.8 \\
35.2 \\
31.9\end{array}$ & $\begin{array}{r}10,500 \\
10,260 \\
10,000 \\
7,760 \\
9,880 \\
10,900 \\
10,700 \\
10,650 \\
11,300 \\
9,695 \\
10,000 \\
9,250 \\
10,200 \\
9,800 \\
9,900 \\
9,500 \\
10,100 \\
10,200\end{array}$ \\
\hline Dousman & $\begin{array}{l}6 \\
7\end{array}$ & 1 & $\cdots$ & (2) & & ........ & .......... & $\cdots \cdots \cdot$ & ….. & .......... \\
\hline enbeulai & 8 & 1 & 88.2 & e4. 5 & 29.0 & 6.5 & 9,060 & 69.0 & 31.0 & 9,700 \\
\hline $\begin{array}{l}\text { Do.. } \\
\text { Do.. }\end{array}$ & $\begin{array}{l}8 \\
8\end{array}$ & $\begin{array}{l}2 \\
3\end{array}$ & $\begin{array}{l}89.4 \\
90.5\end{array}$ & $\begin{array}{l}68.7 \\
67.2\end{array}$ & $\begin{array}{l}25.7 \\
25.3\end{array}$ & $\begin{array}{l}5.6 \\
7.5\end{array}$ & $\begin{array}{r}10,600 \\
9,500\end{array}$ & $\begin{array}{l}72.9 \\
72.7\end{array}$ & & $\begin{array}{l}11,200 \\
10,300\end{array}$ \\
\hline & 8 & 4 & 90.6 & 63.8 & 24.8 & 1i. 4 & & 72.1 & & 9,600 \\
\hline Do... & 8 & 5 & 89.4 & 65.3 & 23.6 & 11.1 & & 73.5 & 26.5 & \\
\hline $\begin{array}{l}\text { Medins... } \\
\text { Do... }\end{array}$ & $\begin{array}{l}9 \\
9\end{array}$ & $\frac{1}{2}$ & $\begin{array}{l}83.8 \\
69.1\end{array}$ & $\begin{array}{r}63.8 \\
43.4\end{array}$ & $\begin{array}{l}28.6 \\
14.2\end{array}$ & $\begin{array}{r}7.6 \\
42.4\end{array}$ & $\begin{array}{l}8,700 \\
3,700\end{array}$ & $\begin{array}{l}69.0 \\
75.3\end{array}$ & 24.7 & $\begin{array}{l}9,450 \\
6,300\end{array}$ \\
\hline & 9 & 3 & 88.8 & 59.8 & 29.4 & 10.8 & 8,400 & 67.1 & & 9,500 \\
\hline & 9 & 4 & 83.4 & 61.7 & 30. & 8. & & 67. & & 10,600 \\
\hline & 9 & & 83.5 & 56.2 & & 14. & & & & \\
\hline Do... & 9 & 6 & 80.0 & 62.6 & 19. & 18. & 7, & & & \\
\hline ond du & 10 & 1 & 83.1 & 61.8 & 26. & 11. & & 69. & & 9,9 \\
\hline & 10 & $\overline{2}$ & 81.4 & 60. & & 15. & & & & 10,4 \\
\hline & 10 & 3 & 79.5 & 43. 7 & & & & & & 6, \\
\hline & 10 & 4 & 80.1 & 55.4 & 27.5 & 17. & & & & \\
\hline & 10 & 5 & 84.0 & 61.1 & 29. & 9. & & 68 & & \\
\hline & 10 & 6 & 88.9 & 70.5 & 21. & & 10 & & & 11,100 \\
\hline hester.. & 11 & 1 & 79.0 & 55. & & 16.3 & & & & 8,770 \\
\hline & 11 & 2 & & & & & & & & 10,600 \\
\hline & 11 & 3 & 78.6 & & 25. & 21. & & & & \\
\hline & 11 & 3 & 77.9 & 47.9 & 23. & 28. & 6,7 & & & 9,4 \\
\hline & 11 & 5 & & 58.1 & & & & & & 9,0 \\
\hline Iendots. & 12 & 1 & & 64. & & 10. & & & & \\
\hline & 12 & 2 & & 60 . & 25. & & & & & \\
\hline & 12 & $\overline{3}$ & & & & & & & & \\
\hline & 12 & 4 & 85.8 & 56.9 & 25.9 & 17. 2 & & 68. & 31 & 9,500 \\
\hline & 12 & 5 & 85. & 54. & 31. & 14. 2 & & & & 9,200 \\
\hline Do.... & 12 & 6 & 88. & 63.1 & 27. & 9.3 & & & & 10,100 \\
\hline irkesan. & 13 & & & 60.8 & 30.1 & 9.1 & 9,500 & 67.1 & 32.9 & 10,400 \\
\hline & 13 & & 67.0 & 45.9 & & 47.0 & & & & \\
\hline $\begin{array}{l}\text { arshall. } \\
\text { inelande }\end{array}$ & 14 & 1 & $\begin{array}{l}b 18.6 \\
\end{array}$ & 60.9 & $\begin{array}{r}27.9 \\
28\end{array}$ & 11. 2 & $\begin{array}{r}9,000 \\
\end{array}$ & 68. 8 & 31.4 & 10,200 \\
\hline Do.................. & $\begin{array}{l}10 \\
15\end{array}$ & $\frac{1}{2}$ & $\begin{array}{l}89.3 \\
91.4\end{array}$ & $\begin{array}{l}66.2 \\
66.3\end{array}$ & $\begin{array}{l}28.3 \\
27.3\end{array}$ & $\begin{array}{l}5.5 \\
6.4\end{array}$ & & & & 10,300 \\
\hline & 15 & 3 & 89.4 & 71.3 & 22.8 & & & & & \\
\hline & 15 & 4 & 90.7 & 66.0 & 28.5 & 5.5 & 9,960 & 69.9 & 30.1 & 10,500 \\
\hline Do.. & 16 & 1 & ..... & . & & & & & andent & .......... \\
\hline & & & & & & & & & & \\
\hline
\end{tabular}

a Huels, F. W., The peat resources of Wisconsin: Wisconsin Geol. and Nat. Hist. Survey Bull. 45, Econ. Series 20, pp. 149-151, 1915 .

o Partly air-dried before the moisture contentwas determined. 
[Survey of 1908.]

\begin{tabular}{|c|c|c|c|c|c|c|c|c|c|c|c|c|c|c|c|c|c|c|c|}
\hline \multirow{2}{*}{$\begin{array}{l}\text { Location (nearest } \\
\text { town or city). }\end{array}$} & \multirow{2}{*}{$\begin{array}{l}\text { Deposit } \\
\text { No. }\end{array}$} & \multirow{2}{*}{\begin{tabular}{|l} 
Air- \\
drying \\
loss.
\end{tabular}} & \multicolumn{6}{|c|}{ Air-dried. } & \multicolumn{6}{|c|}{ As recelved. } & \multicolumn{5}{|c|}{ Moisture-free. } \\
\hline & & & $\begin{array}{l}\text { Mois- } \\
\text { ture. }\end{array}$ & $\begin{array}{l}\text { Volatile } \\
\text { matter. }\end{array}$ & $\begin{array}{l}\text { Fixed } \\
\text { carbon. }\end{array}$ & Ash. & $\mid \begin{array}{c}\text { British } \\
\text { thermal } \\
\text { units. }\end{array}$ & $\begin{array}{l}\text { Sul- } \\
\text { phur. }\end{array}$ & $\begin{array}{l}\text { Mois- } \\
\text { ture. }\end{array}$ & $\begin{array}{c}\text { Volatlle } \\
\text { matter. }\end{array}$ & $\begin{array}{l}\text { Fixed } \\
\text { carbon. }\end{array}$ & Ash. & $\mid \begin{array}{c}\text { British } \\
\text { thermal } \\
\text { units. }\end{array}$ & $\begin{array}{c}\text { Sul- } \\
\text { phur. }\end{array}$ & $\mid \begin{array}{l}\text { Volatile } \\
\text { mstter. }\end{array}$ & $\begin{array}{l}\text { Fixed } \\
\text { carbon. }\end{array}$ & Ash. & $\mid \begin{array}{c}\text { British } \\
\text { thermal } \\
\text { units. }\end{array}$ & $\begin{array}{c}\text { Sul- } \\
\text { phur. }\end{array}$ \\
\hline \multirow{2}{*}{$\begin{array}{r}\text { Madison........... } \\
\text { Do............ } \\
\text { Do............ } \\
\text { Do............. }\end{array}$} & \multirow{8}{*}{\begin{tabular}{|l|}
301 \\
301 \\
302 \\
302 \\
303 \\
303 \\
303 \\
303 \\
304 \\
304 \\
304 \\
305 \\
305 \\
306 \\
306 \\
307 \\
308 \\
308 \\
309 \\
309
\end{tabular}} & \multirow{8}{*}{$\begin{array}{l}61.50 \\
82.00 \\
68.50 \\
84.30 \\
73.80 \\
85.00 \\
6.60 \\
19.40 \\
85.60 \\
13.00 \\
94.90 \\
16.40 \\
83.60 \\
28.40 \\
20.40 \\
86.90 \\
. \cdots \cdots\end{array}$} & \multirow{8}{*}{$\begin{array}{r}9.26 \\
8.60 \\
8.99 \\
6.92 \\
11.99 \\
7.72 \\
9.95 \\
6.95 \\
6.90 \\
6.62 \\
7.64 \\
7.70 \\
7.74 \\
6.82 \\
7.79 \\
9.25 \\
8.22\end{array}$} & 47.18 & 17.89 & 25.67 & 6,250 & 0.42 & \multirow{5}{*}{$\begin{array}{r}65.07 \\
a 83.55 \\
71.33 \\
a 85.39 \\
76.94 \\
a 86.16 \\
15.89 \\
25.00 \\
a 86.59 \\
18.76 \\
a 95.29\end{array}$} & 18.16 & 6.89 & 9.88 & 2,407 & 0.16 & 51.99 & 19.73 & 28.28 & 6,890 & 0.45 \\
\hline & & & & 50.82 & 21.42 & 18.77 & 6,943 & .38 & & - 16.01 & 6.75 & 3.91 & $\dddot{2}, 197$ & iiz & 55.84 & 23.55 & 20.61 & 7,628 & .42 \\
\hline $\begin{array}{r}\text { Fond du Lac...... } \\
\text { Do................... }\end{array}$ & & & & 51.03 & 21.75 & 15.23 & 7,169 & 96 & & 13.37 & 3.70 & 3.90 & “i, & iij & 57.98 & 24.72 & iï. 30 & $\dddot{8}, \mathrm{i} i 0^{\circ}$ & 范 \\
\hline \multirow[t]{2}{*}{ 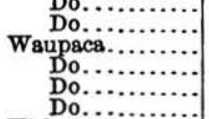 } & & & & $\begin{array}{l}48.10 \\
54.23\end{array}$ & $\begin{array}{l}25.14 \\
23.44\end{array}$ & $\begin{array}{l}10.77 \\
15.38\end{array}$ & $\begin{array}{l}7,468 \\
7,614 \\
7,614\end{array}$ & 7.79 & & $\begin{array}{r}44.96 \\
43.71\end{array}$ & $\begin{array}{l}23.49 \\
18.89\end{array}$ & $\begin{array}{l}15.60 \\
12.40\end{array}$ & $\begin{array}{l}\dddot{6}, 90750 \\
0,130\end{array}$ & $\begin{array}{r}7.74 \\
.30\end{array}$ & $\begin{array}{l}-13.45 \\
58.29\end{array}$ & 25.18 & $\begin{array}{l}10.60 \\
16.53\end{array}$ & $\begin{array}{l}7,203 \\
8,181\end{array}$ & $\because .88$ \\
\hline & & & & 65.31 & 13.63 & 24.44 & 6,970 & & & "78. ï & iii. 86 & 21.20 & $\dddot{6}, \ddot{0}, \ddot{*}$ & $\cdots \cdots$ & 59.23 & iii. $60^{\circ}$ & 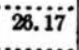 & 7,465 & \\
\hline \multirow{3}{*}{ 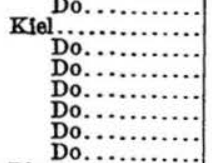 } & & & & 48.38 & 16.00 & 27.92 & 6,107 & ii.si & $\begin{array}{r}22.84 \\
a 84.82\end{array}$ & 40.45 & 13.37 & 23.34 & $\dddot{5}, \mathrm{iö}$ & i. 20 & 52.42 & ii. 33 & 30.25 & 6,619 & i. 63 \\
\hline & & & & $\begin{array}{l}53.30 \\
53.04\end{array}$ & $\begin{array}{l}20.42 \\
20.85\end{array}$ & $\begin{array}{l}19.46 \\
18.32\end{array}$ & $\begin{array}{c}\dddot{7}, 256 \\
7,119 \\
\ldots \ldots \ldots\end{array}$ & $\begin{array}{r}1.313 \\
.82\end{array}$ & $\begin{array}{r}33.82 \\
2680 \\
a 88.11\end{array}$ & $\dddot{37.45}$ & $\begin{array}{l}75.34 \\
16.60\end{array}$ & $\begin{array}{l}13.39 \\
14.58\end{array}$ & $5,7,395$ & i. 08 & 56.52 & 22.69 & $\begin{array}{l}20.88 \\
10.88\end{array}$ & $7,7,787$ & i. \\
\hline & & & & $\because 38.07$ & 23. 21 & iio. & 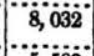 & 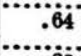 & $\begin{array}{r}20.43 \\
091.06\end{array}$ & 70.35 & 20.12 & 9. & "̈, & .550 & 63.28 & 25.28 & iii. 44 & 8,753 & $\dddot{60}$ \\
\hline \multirow{2}{*}{ 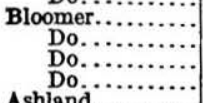 } & \multirow{9}{*}{$\begin{array}{l}310 \\
310 \\
311 \\
311 \\
312 \\
312 \\
313 \\
313 \\
314 \\
314 \\
315 \\
315 \\
316 \\
316 \\
317 \\
317 \\
318 \\
318\end{array}$} & \multirow{9}{*}{$\begin{array}{l}90.30 \\
72.90 \\
80.10 \\
75.80 \\
82.20 \\
45.30 \\
61.30 \\
9.00 \\
90.80 \\
11.30 \\
91.10 \\
4.90 \\
92.50 \\
9.30 \\
90.50 \\
7.80 \\
88.50 \\
10.10 \\
93.70\end{array}$} & $\begin{array}{r}11.76 \\
9.15\end{array}$ & $41.80^{\circ}$ & i5. $30^{\circ}$ & 31. ii & 3,503 & 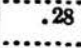 & $\begin{array}{r}76.09 \\
a 81.92\end{array}$ & iii.33 & 4.114 & $\because \dddot{40}$ & $i, 490^{\circ}$ & $\because 08$ & 47.39 & iï.3i & 35.30 & $\dddot{6}, 233$ & $\because 33$ \\
\hline & & & $\begin{array}{r}10.35 \\
7.67\end{array}$ & 49.26 & 24.74 & 15.65 & 7,020 & $2 \mathrm{ii}$ & $\begin{array}{r}78.32 \\
a 83.57\end{array}$ & ii. 92 & 3.90 & $3.79^{\circ}$ & "i, & .05 & 54 & $27.60^{\circ}$ & iit. 47 & 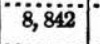 & .23 \\
\hline 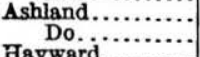 & & & $\begin{array}{l}6.82 \\
4.57\end{array}$ & $\begin{array}{l}26.69 \\
22.60\end{array}$ & $\begin{array}{l}6.38 \\
5.64\end{array}$ & $\begin{array}{l}60 . \mathrm{ii} \\
67.19\end{array}$ & $\begin{array}{r}2,984 \\
\ldots \ldots\end{array}$ & 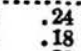 & $\begin{array}{r}49.03 \\
063.07\end{array}$ & $\begin{array}{r}7 i .60 \\
8.75\end{array}$ & $\begin{array}{l}{ }_{3.19}^{3.49} \\
2.18\end{array}$ & $\begin{array}{l}32.88 \\
26.00\end{array}$ & $\because i, 633$ & $\because: 07$ & $\begin{array}{l}28.64 \\
23.69\end{array}$ & $\begin{array}{l}76.05 \\
5.91\end{array}$ & $\begin{array}{l}64.951 \\
70.40\end{array}$ & $\dddot{3}, 200$ & $\because .28$ \\
\hline ywa & & & $\begin{array}{r}10.21 \\
6.66\end{array}$ & 50.77 & 25.06 & $\begin{array}{l}13.96 \\
\ldots \ldots\end{array}$ & 7,866 & .28 & $\begin{array}{r}18.29 \\
a 91.41\end{array}$ & 46.20 & 22.81 & $\begin{array}{l}12.70 \\
100\end{array}$ & $\dddot{7}, \mathrm{i} ; \mathrm{i}$ & .24 & 56.54 & 27.92 & 15.54 & $876 \mathrm{i}^{\circ}$ & .29 \\
\hline & & & $\begin{array}{l}7.71 \\
3.93\end{array}$ & $\begin{array}{l}51.13 \\
\ldots \ldots \ldots\end{array}$ & 20.07 & 21.09 & 7,4 iii & .39 & $\begin{array}{l}18.14 \\
a 91.45\end{array}$ & 45.35 & 17.80 & $18.7 \mathrm{i}$ & $6,577^{\circ}$ & .35 & 55.40 & 21.74 & 22.80 & $\dddot{8}, 028$ & 73 \\
\hline $\begin{array}{l}\text { ew Auburn... } \\
\text { Do.......... }\end{array}$ & & & $\begin{array}{l}7.66 \\
4.95\end{array}$ & 53.55 & 25.48 & i3.3i & 20 & 20 & $\begin{array}{l}12.18 \\
a 92.87\end{array}$ & 30.93 & 2403 & $12.66^{\circ}$ & $7,810^{\circ}$ & "ii & 57.99 & $\dddot{27.59}$ & ii. 42 & $\dddot{8}, 905$ & .20 \\
\hline $\begin{array}{l}\text { meron............ } \\
\text { Do. }\end{array}$ & & & $\begin{array}{l}5.21 \\
7.22\end{array}$ & 60.08 & 26.78 & 7.93 & $\dddot{8}, 7 i 0^{\circ}$ & .28 & $\begin{array}{r}14.03 \\
a 91.19\end{array}$ & 54.40 & 2420 & 7.10 & 7,906 & $.25^{\circ}$ & 63.38 & 28.26 & 8.36 & 9,196 & .20 \\
\hline odysmith .............. & & & $\begin{array}{l}3.85 \\
5.31\end{array}$ & 5400 & 20.98 & $2 i .17$ & 7,640 & .24 & $\begin{array}{r}11.35 \\
a 89.11\end{array}$ & $40.79^{\circ}$ & 19.34 & iig. 52 & $\dddot{7} \dddot{7}, 0$ osi & 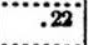 & 30.16 & $2 i .82$ & $\dddot{2} 20$ & $\dddot{7}, 932$ & $\because \ddot{20}$ \\
\hline $\begin{array}{r}\text { Heafford Junction } \\
\text { Do.................. }\end{array}$ & & & $\begin{array}{l}4.07 \\
5.08\end{array}$ & 53.63 & 20.44 & 21.86 & 7,722 & .25 & $\begin{array}{r}13.76 \\
a 94,02\end{array}$ & 48. $2 \mathrm{ii}$ & 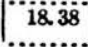 & 19.65 & 6,943 & .21 & 35.90 & 2ii.3ï & 22.79 & $\dddot{8} \dddot{8}, 05 i$ & $\because \ddot{2 A}$ \\
\hline
\end{tabular}




\begin{tabular}{|c|c|c|c|c|c|c|c|c|c|c|c|c|c|c|c|c|c|c|c|}
\hline 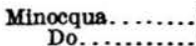 & $\begin{array}{l}319 \\
319\end{array}$ & $\begin{array}{l}15.10 \\
83.20\end{array}$ & $\begin{array}{l}5.72 \\
4.12\end{array}$ & 50.08 & 20.82 & 23.38 & 7,456 & .28 & $\begin{array}{r}19.96 \\
a 83.89\end{array}$ & 42.52 & 17.67 & 19.85 & 6,331 & .24 & 53.12 & 22.08 & 24.80 & 7,909 & 30 \\
\hline $\mathrm{D}$ & $\begin{array}{l}519 \\
320 \\
320\end{array}$ & 9.20 & $\begin{array}{l}4.12 \\
4.58\end{array}$ & 51.92 & 18. 82 & 24.68 & 7,236 & .50 & $\begin{array}{r}13.89 \\
0.96 \\
\end{array}$ & 47.14 & 17.09 & 22.41 & 6,570 & .45 & $544 i$ & 19.72 & 25.87 & $7, \overline{5} 83$ & .58 \\
\hline c du Flan & 321 & 10.80 & $\begin{array}{l}2.60 \\
5.62\end{array}$ & 53.52 & 22.84 & 20.96 & $\dddot{8}, 417$ & .16 & 13.19 & 47.47 & 20.37 & 18.70 & 7,508 & .14 & $54.90^{\circ}$ & 23.47 & 21.54 & 8,649 & ii \\
\hline owell...... & $\begin{array}{l}321 \\
322\end{array}$ & $\begin{array}{l}83.70 \\
11.40\end{array}$ & $\begin{array}{l}5.02 \\
6.56\end{array}$ & $\dddot{53} .04$ & $\dddot{25.32}$ & 10.08 & 7,627 & .89 & $\begin{array}{r}a 84.52 \\
17.21\end{array}$ & 46.99 & 22.44 & 13. 36 & $\dddot{6}, 757$ & .79 & 56.76 & $\dddot{27} .10$ & ï.ïi & $\dddot{8}, \mathrm{i} 6 \mathrm{i}$ & نे \\
\hline Do & 322 & $\begin{array}{r}87.50 \\
9.00\end{array}$ & $\begin{array}{l}6.29 \\
4.62\end{array}$ & 58.33 & 25.76 & iii. 29 & 8,723 & .22 & 13. & 58.08 & 23.45 & 10.27 & 7,938 & .20 & 61.15 & 27.02 & ii. 83 & $\dddot{9}, 146$ & $\dddot{203}$ \\
\hline ark Fail & $\begin{array}{l}323 \\
324 \\
204\end{array}$ & $\begin{array}{r}91.80 \\
8.00\end{array}$ & $\begin{array}{l}6.3 \\
3 .\end{array}$ & 42.81 & 18.95 & 34.25 & $\dddot{6}, 291$ & .30 & 11. & 39.39 & 17.43 & $31.3 i$ & 75,787 & .28 & 44.59 & 19.74 & 35.67 & 6,552 & .32 \\
\hline ewau & 325 & 6.10 & $\begin{array}{l}5.96 \\
8.96\end{array}$ & 53.94 & 23.60 & 13.50 & 7,290 & .47 & 14. & 50.65 & 22.16 & 12.68 & 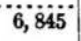 & .44 & 39.25 & 25,92 & 14.83 & 8,006 & .51 \\
\hline lgoms & 325 & & $\begin{array}{l}7.88 \\
7.51\end{array}$ & 38.52 & 15.19 & 38.78 & 3,445 & .26 & 10.75 & 37.17 & 14.66 & 37.42 & 5,254 & .25 & 41.65 & 16.42 & 41. 93 & $\dddot{5}, \ddot{886}$ & .28 \\
\hline turge & $\begin{array}{l}326 \\
327\end{array}$ & 67. & $\begin{array}{l}7.63 \\
9.01 \\
9\end{array}$ & 54.73 & 16.20 & 20.06 & 7,186 & .77 & & 51.28 & 15.18 & $18.80^{\circ}$ & 6,734 & .72 & 60.15 & 17.80 & 22.05 & 7,898 & "84 \\
\hline $\begin{array}{l}\text { De } \\
\text { eshti } \\
\text { embi }\end{array}$ & $\begin{array}{l}327 \\
328 \\
329\end{array}$ & $\begin{array}{r}91.40 \\
75.10 \\
6.80\end{array}$ & $\begin{array}{l}8.77 \\
5.05 \\
9.25\end{array}$ & $\begin{array}{r}33.29 \\
50.60\end{array}$ & $\begin{array}{l}18.69 \\
23.15\end{array}$ & $\begin{array}{l}32.95 \\
17.00\end{array}$ & $\begin{array}{l}6,012 \\
7,112\end{array}$ & $\begin{array}{r}.66 \\
1.51\end{array}$ & $\begin{array}{r}a 92.15 \\
76.36 \\
15.42\end{array}$ & $\begin{array}{l}10.78 \\
47.16\end{array}$ & $\begin{array}{r}4.66 \\
21.58\end{array}$ & $\begin{array}{r}8.20 \\
15.84\end{array}$ & $\begin{array}{l}1,498 \\
6,628\end{array}$ & $\begin{array}{l}.16 \\
1.41\end{array}$ & $\begin{array}{l}45.60 \\
55.75\end{array}$ & $\begin{array}{l}19.71 \\
25.52\end{array}$ & $\begin{array}{l}34.69 \\
18.73\end{array}$ & $\begin{array}{l}\dddot{6}, 334 \\
7,835\end{array}$ & $\begin{array}{l}0.68 \\
1.67\end{array}$ \\
\hline$\underset{\text { ager }}{\mathrm{D}}$ & $\begin{array}{l}329 \\
330\end{array}$ & $\begin{array}{l}82.80 \\
10.30\end{array}$ & $\begin{array}{l}6.80 \\
5.71\end{array}$ & “ & $\dddot{26.13}$ & 8.97 & $\dddot{8}, \dddot{856}$ & .21 & 7 & 53.09 & 23.44 & 8.05 & 7,943 & .19 & 62.77 & 27.71 & 9.52 & $\dddot{9}, \dddot{39} i^{\circ}$ & $\dddot{2} 20$ \\
\hline Do. & $\begin{array}{l}330 \\
331\end{array}$ & $\begin{array}{l}86.4 \\
11.2\end{array}$ & $\begin{array}{l}5.7 \\
5.2\end{array}$ & 60.14 & 2406 & 10.59 & 8,869 & .26 & $\begin{array}{r}a 87.18 \\
15.83\end{array}$ & 53.40 & 21.37 & 9. 40 & 7,875 & .23 & 63.44 & 25.39 & 11.17 & $\dddot{9}, \dddot{3} 30$ & $\ddot{2} \ddot{27}$ \\
\hline & 331 & & 5.1 & & & & & & a86. & & & & & & & & & & \\
\hline $\begin{array}{l}\text { itige } \\
\text { punt }\end{array}$ & $\begin{array}{l}332 \\
333\end{array}$ & $\begin{array}{l}78 . \\
12 .\end{array}$ & $\begin{array}{l}7.6 \\
7.9\end{array}$ & $\begin{array}{l}43.04 \\
54.46\end{array}$ & $\begin{array}{l}19.47 \\
25.47\end{array}$ & $\begin{array}{l}29.82 \\
12.12\end{array}$ & $\begin{array}{l}5,870 \\
7,191\end{array}$ & $\begin{array}{l}.60 \\
.69\end{array}$ & $\begin{array}{l}80.24 \\
19.82\end{array}$ & $\begin{array}{r}9.21 \\
47.43\end{array}$ & 4.17 & $\begin{array}{r}6.38 \\
10.56\end{array}$ & $\begin{array}{l}1,256 \\
6,264\end{array}$ & .13 & 59.15 & $\begin{array}{l}21.10 \\
27.68\end{array}$ & $\begin{array}{l}32.29 \\
13.17\end{array}$ & $\begin{array}{l}6,358 \\
7,812\end{array}$ & $\begin{array}{l}.66 \\
.75\end{array}$ \\
\hline & 333 & & 7.24 & & & & & & $a 87.57$ & & & & & & & & & & \\
\hline
\end{tabular}

a Shipped to the laboratory in air-tight containers. 
Analyses of peat and muck. ${ }^{a}$

[F. M. Stanton, U. S. Geological Survey, anslyst.]

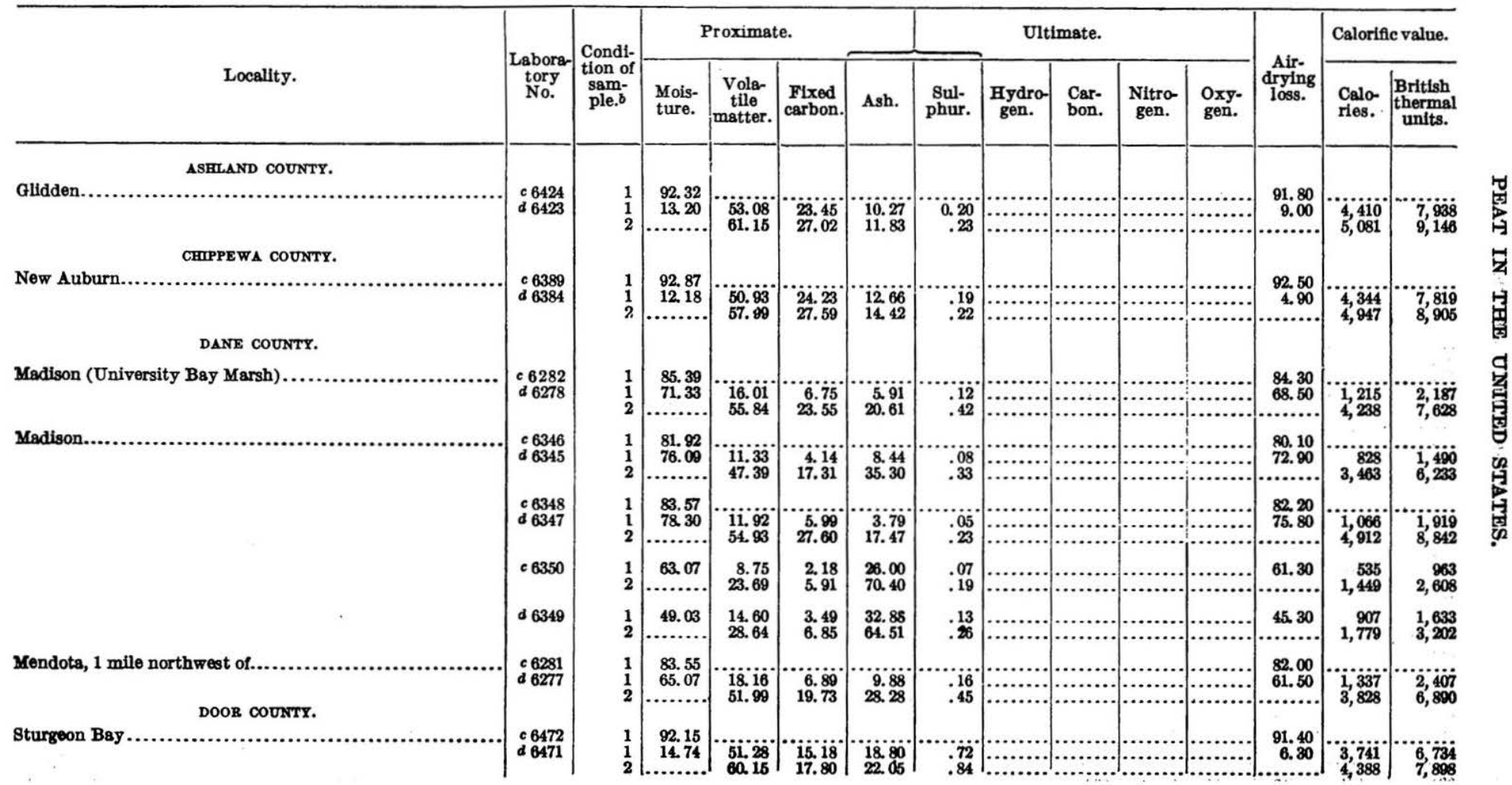




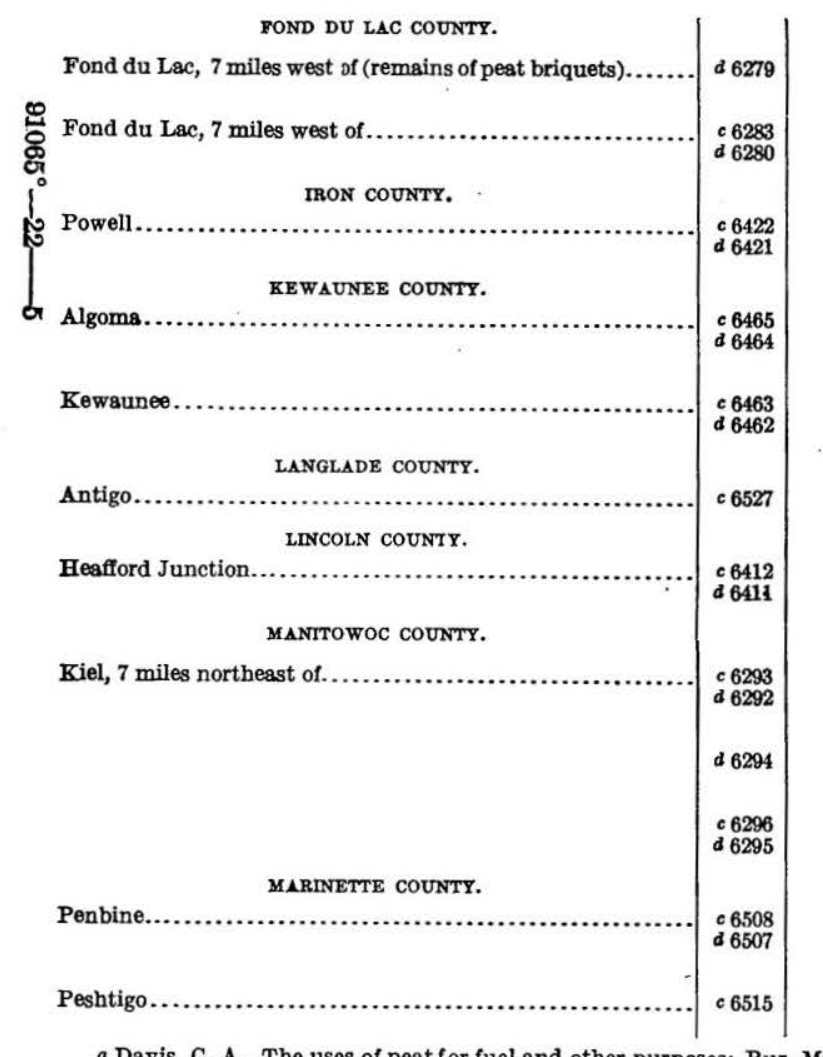

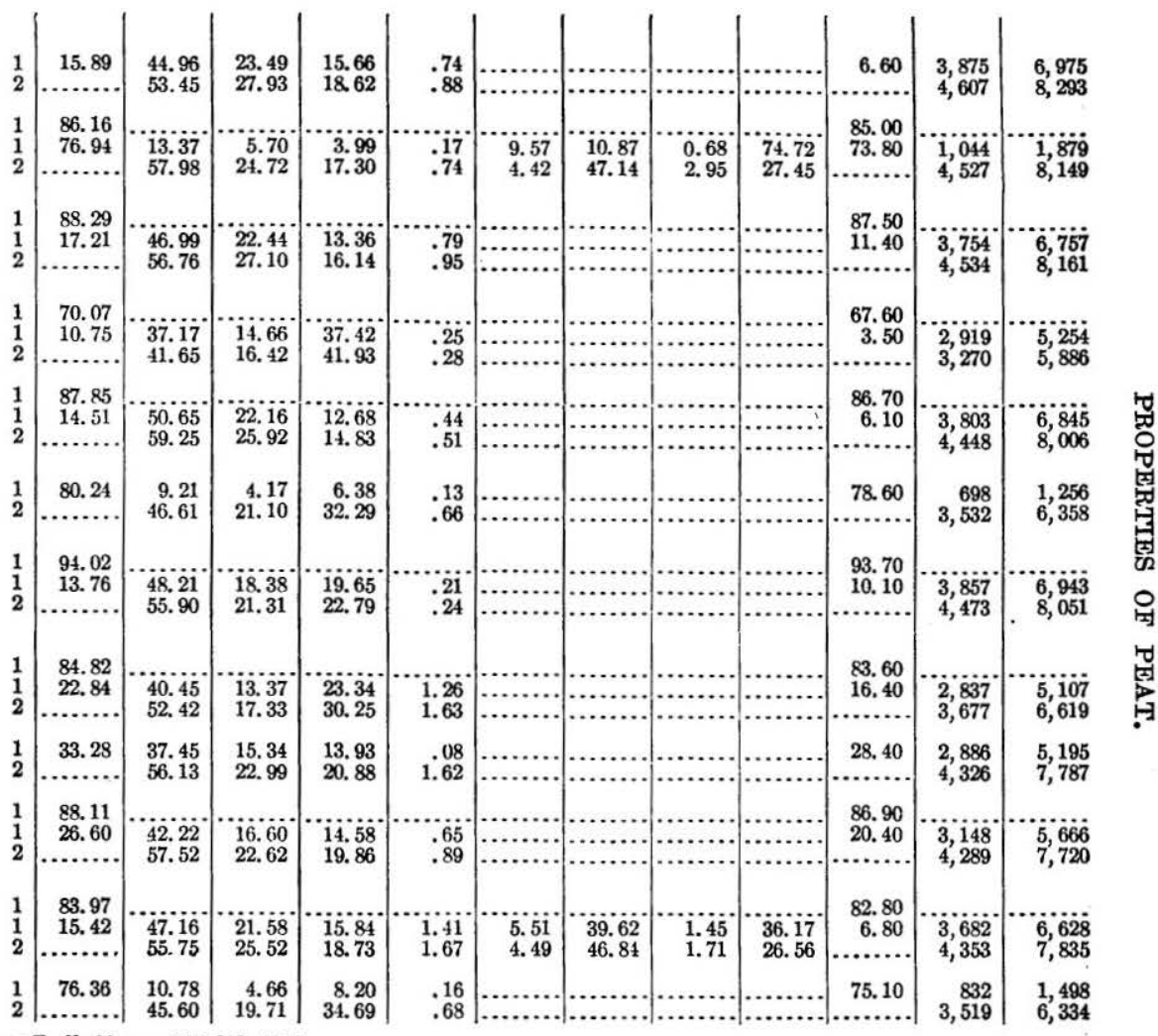

$a$ Davis, C. A., The uses of peat for fuel and other purposes:
$b 1$, Native peat. 2, Moisture-free peat. 
Analyses of peat and muck-Continued.

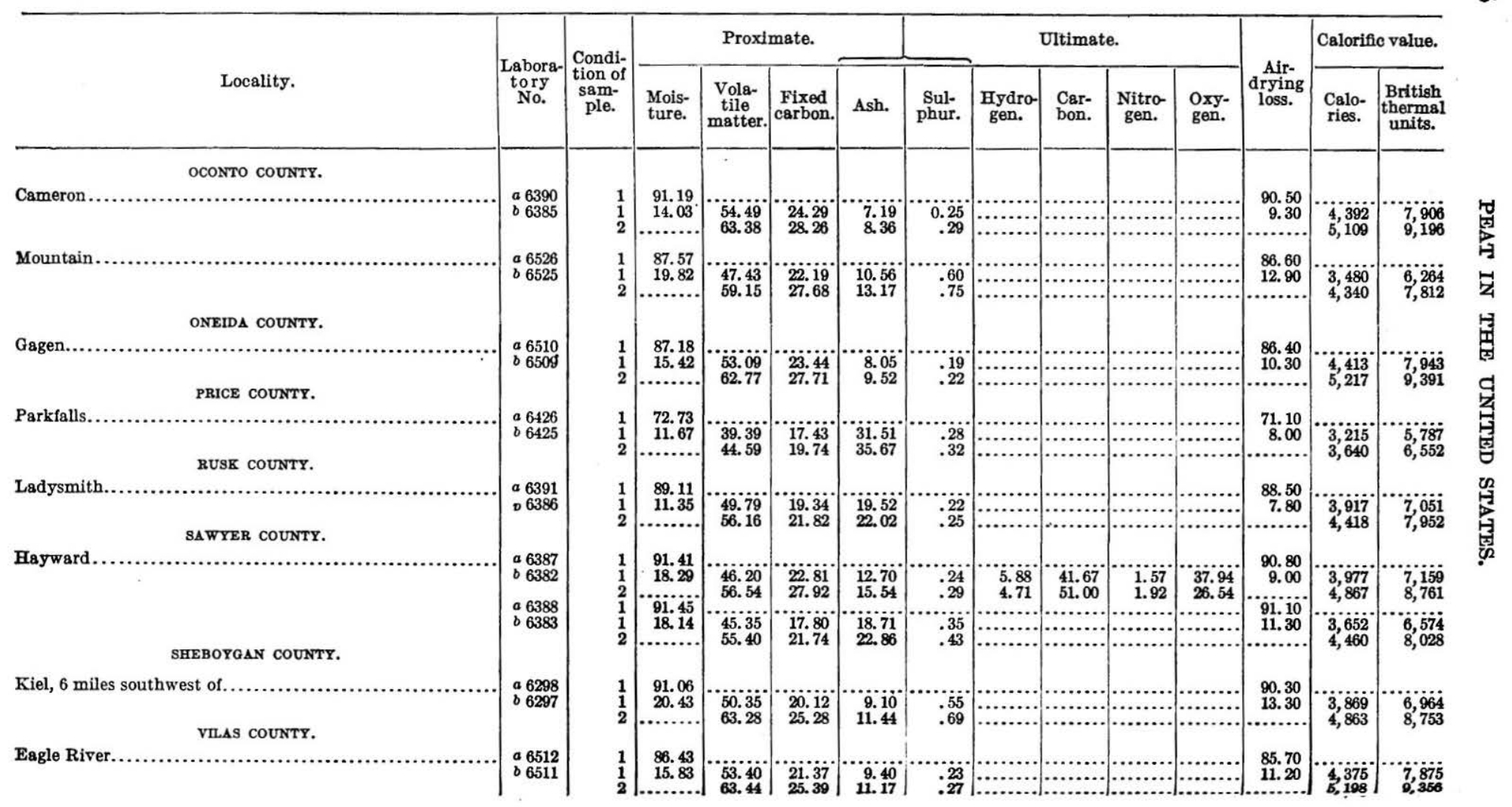




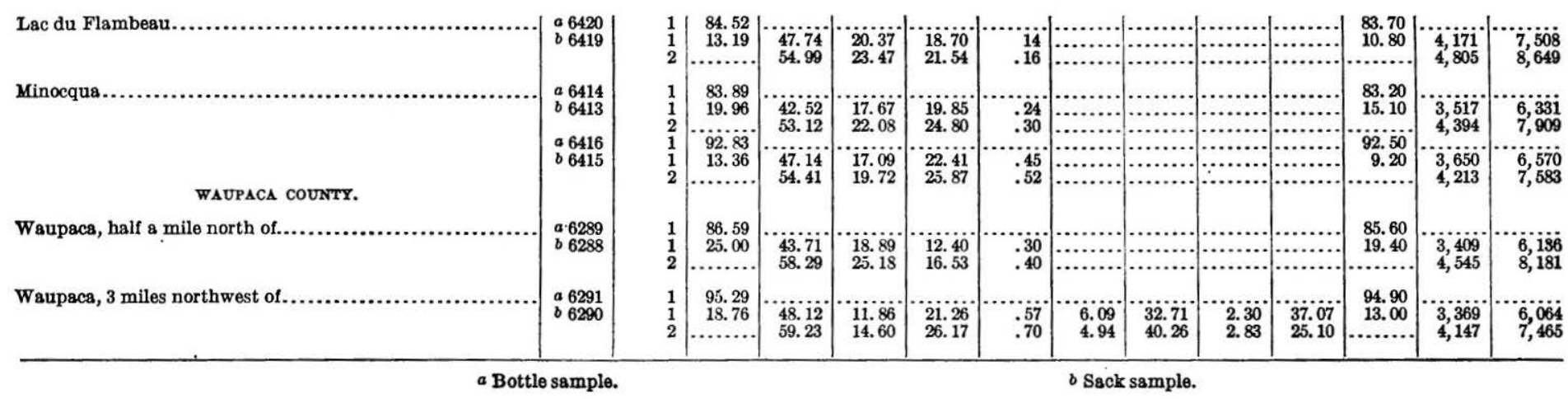




\section{CONCLUSIONS.}

Minnesota, Wisconsin, and Michigan contain a large quantity of high-grade peat. The average nitrogen content of 246 samples, collected from widely scattered localities in Minnesota, was 2.25 per cent and the average calorific value of the moisture-free peat was 8,329 British thermal units. The average composition of Wisconsin peat and muck is shown by the following table, compiled in part from the foregoing analyses:

Average composition of peat and muck from Wisconsin.

Moisture (native)
Moisture (air-dried)
Volatile matter-16.5
Fixed carbon
Ash
Sulphur
Carbon (combined)
Hydrogen
Oxygen
Nitrogen

Although the peat deposits of Michigan have not been so thoroughly tested as those of Minnesota and Wisconsin, they are of high quality.

Large quantities of good peat, suitable for fertilizer, fuel, packing material, and other products are found in all the States shown in the tables. Specific localities throughout the United States where peat suitable for the purposes mentioned may be obtained are listed on pages $91-202$.

Some of the peat deposits of New York and northern New Jersey are similar in chemical properties to those of Wisconsin and Michigan and are well adapted for the manufacture of peat products.

The peats of many deposits in Iowa, Illinois, and Pennsylvania contain too much ash for use as fuel, though they may be used for making fertilizer and packing material. Of 266 samples of Iowa peat selected at random and analyzed, only 15 contained less than 25 per cent of inorganic material and only 1 less than 15 per cent. The peats of the Illinois and Pennsylvania deposits are also relatively impure. However, the average nitrogen content of the Illinois peat as shown by analyses of samples is 2.65 per cent, and the peats of Iowa and Pennsylvania probably contain about 2 per cent, which is the general average. Five samples of Illinois peat contain more than 3 per cent of nitrogen and one from the Duck Lake deposit in that State contains 3.84 per cent, an unusually high percentage for raw peat.

The average nitrogen content of peat from Indiana, as shown by the foregoing analyses, is 2.8 per cent, and the average calorific value 
is 8,552 British thermal units. The peat of Ohio ranges in content of nitrogen from 1.01 to 3.60 per cent and in calorific value (moisturefree peat) from 7,132 to 9,736 British thermal units. It is therefore apparent that the deposits of these States contain peat well adapted to use both as fertilizer and as fuel.

Aside from some of the peat deposits of Massachusetts and Rhode Island and a few in other States that contain a large quantity of ash, most of the peat in the New England region is of good quality. In 69 samples of peat from Maine the range in calorific value of the moisture-free material was from about 8,000 to 9,800 British thermal units. Maine also contains an immense quantity of sphagnum moss and moss peat suitable for surgical dressings, packing material, and stable litter.

The peat deposits of the Atlantic coastal region south of northern New Jersey, as well as those in the Pacific Coast States, are variable in quality. The salt-marsh peat is generally too impure for commercial use, but some of the inland swamps, like the Dismal Swamp of Virginia and North Carolina and the Everglades of Florida, contain good peat. In fact, peat from Florida compares favorably with peat from any other part of the country. It is equal to the best peat in the Great Lakes States and is higher in calorific value and lower in ash than much of the peat of New England.

\section{USES OF PEAT.}

\section{AGRICULTURAI USES.}

The uses of peat in agriculture are manifold. Prepared peat is used directly as a fertilizer, as an ingredient of commercial fertilizers and stock feed, as stable litter, and as an absorbent and disinfectant. Peat soils are well adapted to the growth of certain crops.

FERTIIIZER.

GENERAL FEATURES.

The peat deposits of the United States form one of the few extensive known domestic sources of nitrogen that can be converted into plant food at a price low enough to be economically used by the farmers. The average nitrogen content of domestic air-dried peat is about 2 per cent, although many peats contain more than this quantity. This nitrogen may be recovered in the form of ammonium sulphate by the methods considered on pages 72-73 or may be made available for plant food without extracting it from the peat. Arguments are often advanced against the direct use of peat as a source of nitrogen in soil fertilization, because not all the nitrogen it con- 
tains, as shown by chemical analysis, is readily available for plant food, but this criticism seems to be based on a misconception of the nature of the peat. It is true that only a part of the nitrogen shown by analysis can be immediately used as food by plants, but it is equally true that a chemical analysis of peat is not a fair test of its value as a fertilizer and that the total quantity of potential soluble nitrogen formed and released by bacterial action from time to time after the peat has been applied to the soil is in the aggregate often greater than the percentage found in some commercial fertilizers. Fortunately all the nitrogen in peat is not soluble at one time, or it would leach out, and the potentially rich black peat soils of this country would become unproductive.

Bacterized peat as a direct fertilizer is said to be even a more prolific source of soluble nitrogen than the crude material. The following method of increasing the nitrogen content of soils by means of peat is proposed by Bottomley: ${ }^{5}$

It is well known that if peat is exposed to the air for several years it is neutralized by the formation of ammonia, and a large proportion of the insoluble material is converted into food available for plant life. By inoculating the peat with aerobic bacteria it is found possible greatly to accelerate this change and to increase materially the quantity of plant food. The problem, however, was not to discover a fertilizer, but to find a medium in which nitrogen-fixing organisms could be cultivated and placed on the soil. This medium is found in the peat treated with aerobic bacteria. To prepare it for inoculation the peat is kept moist at a temperature of $25^{\circ} \mathrm{C}$. for about a week. Steam is then forced through it to insure that all organisms, bacterial or otherwise, are destroyed, and the result is a sterile medium, neutral or slightly alkaline, suitable for the cultivation of plants or of nitrifying bacteria. The sterilized peat is then inoculated with a mixed culture of Bacillus radioicola and Azotobacter chroococcum, which multiply rapidly and soon permeate the entire culture bed. After complete saturation the bacterial growth is arrested by drying the peat, and it is then ready for use.

It is said that the bacteria in this material enrich the soil to which they are applied by extracting nitrogen from the air and converting it into soluble plant food, and that owing to continuous bacterial action frequent subsequent treatment is unnecessary. According to some reports, however, the process seems to be of doubtful value.

Another process for the commercial application of bacteria to soil fertilization is reported by John N. Hoff, of New York. According to his method the peat is cultivated for several seasons, excavated, sun dried, neutralized or made slightly alkaline, and used for a carrier and energizer of several varieties of nitrogen-fixing bacteria. When mixed with certain phosphates and applied to the soil these

\footnotetext{
${ }^{5}$ Knox, G. D., The spirit of the soil, 242 pp., 17 flgs., London, Constable \& Co. (Ltd.),
} 1916. 
bacteria are said to stimulate plant growth and to react upon and release phosphorus from insoluble chemical combinations.

According to reports bacterized peat is being used commercially in England. It is said that larger crops have been grown upon soil enriched by bacterized peat in the United States than could have been obtained from the same land treated with commercial fertilizer.

Whether used as a direct fertilizer or as an ingredient of commercial ferilizers, peat when properly treated is valuable both chemically and physically. Its content of soluble nitrogen is immediately available for plant food, and it is potentially rich in this element, which gradually forms soluble compounds and is released; it supplies humus, a vital requirement for plant life under natural conditions; on account of its black color it absorbs heat; soils to which it is applied are made friable and can be readily worked; and its water-holding properties are proverbial. Because of these characteristics peat is being used more and more in soil cultivation.

For those who propose to enter this branch of the peat industry it is suggested that caution be observed in selecting a suitable deposit. Before any money is invested a careful survey should be made of the prospective deposit to insure that there is a sufficient quantity of peat to justify the erection of a plant. Typical samples should be taken from different parts of the deposit and examined to determine whether the material is chemically adapted for fertilizer. Many peats are acid when first taken from the bog or swamp.

Black, well-humified peat is most satisfactory for soil fertilization. Only bogs containing peat that is rich in nitrogen should be selected. The acidity of the raw peat must be corrected by thorough aeration and liming before any attempt is made to market it as a fertilizer for ordinary crops. One of the great handicaps of the peat-fertilizer industry in this country has been the lack of uniformity in its product, and as the success of a plant depends upon the character of the peat used, too much caution can not be observed in the selection of the material.

\section{METHODS OF PREPARATION.}

Equally important as the kind of peat are the process and machinery employed in treating it. The deposit must first be drained and cleared of trees, brush, and turf. Cultivation of the peat for several seasons will correct the acidity and afford means for determining its agricultural value. After the upper layer has been plowed, disked, and harrowed, the peat is excavated to a depth of about 3 feet and left in windrows on the surface of the bog. When the moisture has been reduced to about 50 per cent by air-drying the material is scraped into piles, loaded into cars, and hauled to a stock pile. After aerobic fermentation is well advanced, the material is 
run through heated rotary driers until the moisture is lowered to 10 per cent, screened, and cooled. The resulting product is used as a nitrogenous ingredient of commercial fertilizers. This material may be further enriched with nitrogen by liming and appropriate inoculation, and if a complete direct fertilizer is desired potash, phosphates, and other minerals are mixed with it.

The outdoor equipment consists essentially of agricultural implements-excavators, scrapers, loaders, some light rails, a few car: and small locomotives, an elevator to raise the peat to the top of the stock pile, and a conveyer for transporting it to the driers. If possible the excavators, scrapers, and loaders should be electrically propelled and operated with caterpillar drives, as machinery so equipped gives little trouble on boggy surfaces. The indoor equipment consists of engines, boilers, dynamos, rotary driers, and sifters. The building containing the drying plant should be fireproof and should be located as near the deposit as possible.

In order that the plant may run throughout the year, it is the best practice to excavate and pile up as much peat as possible during the air-drying season and to complete the drying artificially when the material is needed. By adopting the process and equipment described lost motion is minimized and a large proportion of the water in the peat is eliminated in the field.

Peat fertilizer may be cheaply prepared by farmers owning small bogs by composting the raw peat with manure, and after the bacteria have saturated the mixture it may be applied to the soil in the same way as manure. Land that is deficient in humus and nitrogen will thus be materially benefited.

PEAT SOILS.

GENERAL FEATURES.

Peat soil, when properly treated, is one of the most fertile types of soil in this country. Owing to the abundance of well-drained land that could be more readily tilled, areas of peat and muck have in times past been neglected, but with the rapid increase of our population and the necessity for intensive soil cultivation the attention - of agriculturists and others is now being directed to these lands. In the eastern section of this country there are approximately $15,000,000$ acres of peat and muck land, which supports a growth of shrubs, tamarack, white cedar, birch, water maple, gum, and cypress, and only about 750,000 acres, or 5 per cent of the total area, has been reclaimed for agricultural purposes. Franklin K. Lane, in advocating the reclamation of the swamp and cut-over timber lands of the United States and their sale on the basis of deferred payments 


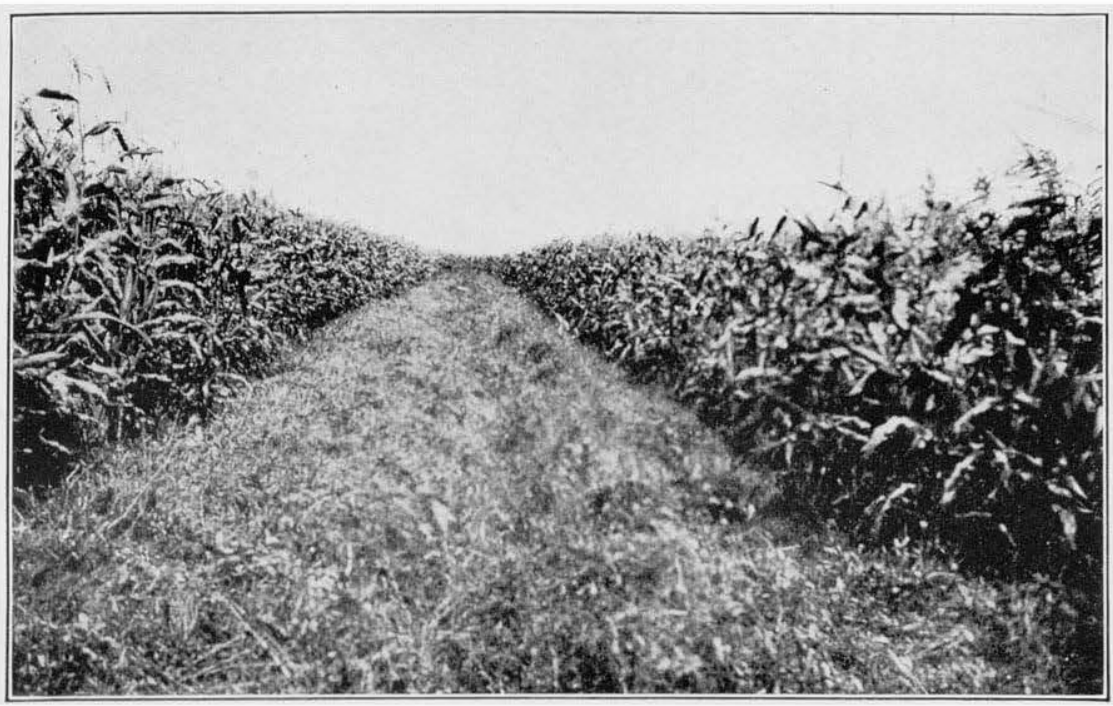

A. EXCELLENT STAND OF CORN RAISED ON RICLAIMED PEAT LAND AT BIG ISLAND, ORANGE COUNTY, N. Y.

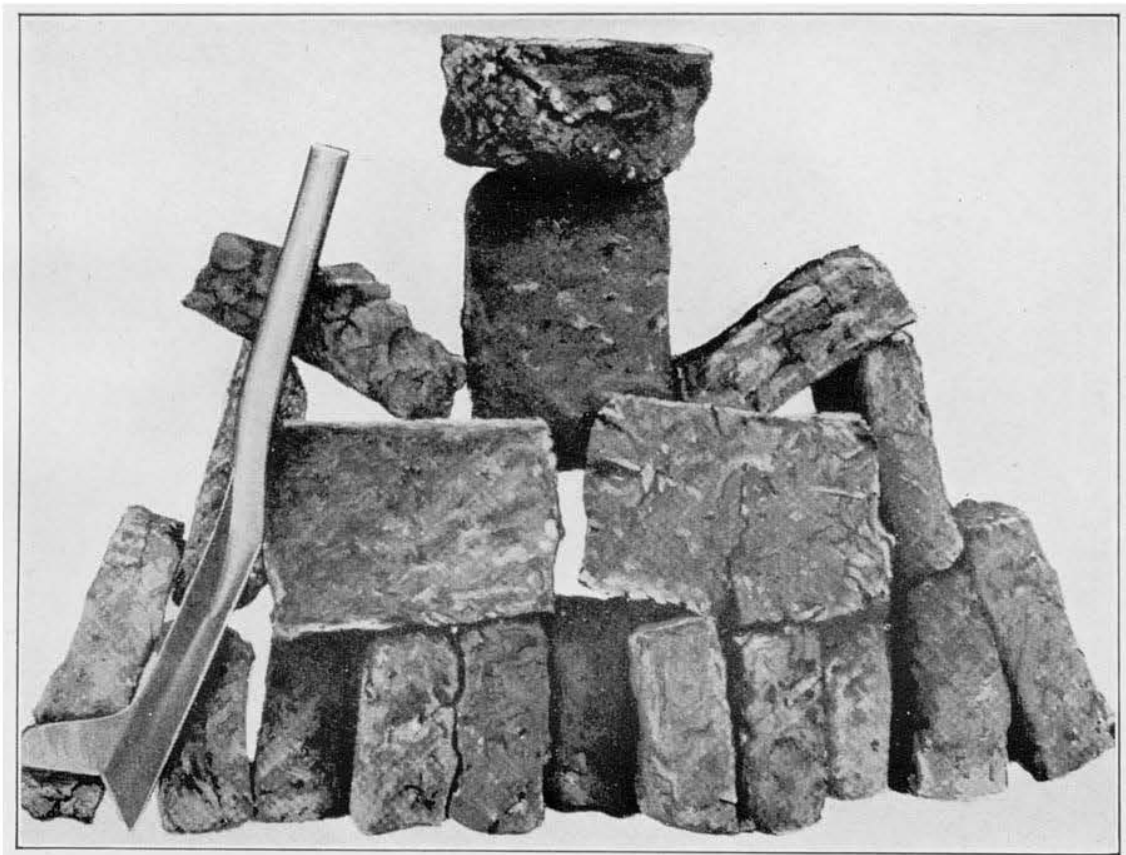

B. DRY HAND-CUT PEAT FUEL AND SLANE WITH WHICH IT IS EXCAVATED.

Commercial Products of Peat. 

to men who had done military service, said in substance in a statement for the press:

There are approximately $200,000,000$ acres of cut-over or logged-off timberland in the eastern half of the United States, and, overlapping to a certain extent the cut-over lands, some $80,000,000$ acres of swamp land, located largely in the southeastern section of the country. To the ordinary observer the reclamation of these waste areas may seem difficult. The soil, howeyer, is in many localities very fertile. For many years leaf mold containing humus has been accumulating, and once the stumps have been blown out and the land cleared of brush and brought under the plow, it is believed that these lands will prove among the most fertile of our agricultural resources. Like the Dismal Swamp in Virginia or the Everglades in Florida, most of the swamp land in its present condition is unfit for human inhabitants. Consider this land cleared of its timber, adequately drained, and intensively cultivated, and some idea of its possibilities for the production of food will be obtained.

Raw peat soils, though too acidic for ordinary farming, after they have been drained, cleared, freely aerated, and properly fertilized, are especially well adapted for the production of vetch, redtop, millet, buckwheat, oats, corn, rye, the potato, the cranberry, the blueberry, the strawberry, and other acid-tolerant crops. ${ }^{6}$. (See Pl. IV, A.) If properly treated with potash salts and with lime they are neutralized or made slightly alkaline and should then yield good crops of red clover, timothy, bluegrass, wheat, rutabagas, and other alkaline or neutral soil crops. The greatest values derived from the cultivation of peat, however, have arisen from its use as a truck crop soil. According to the Bureau of Soils, ${ }^{7}$ cabbage, onions, celery, lettuce, spinach, carrots, beets, turnips, and peppermint are the most valuable crops that are grown on treated areas of peat. The acreage values of these crops so far surpass those of the general farm crops that their cultivation should be made the object of the reclamation of any large areas of peat.

The fertility of peat soil is chiefly due to its content of nitrogen and humus, to its affinity for moisture, and to its blackness, which enables it to absorb heat. For the profitable production and sale of both general and special crops it is desirable that the areas of peat which are close to large city markets and accessible to good transportation and cheap labor should be reclaimed first.

The first attempts to use peat lands for the growth of crops were made in the older agricultural districts of the Eastern States, notably New York and New Jersey. Some of the most productive farm lands in those States were formerly peat bogs and swamps. The truck farm of John N. Hoff near Great Meadows, Warren County,

- Coville, F. V.,. The agricultural utilization of acid lands by means of acid-tolerant crops: U. S. Dept. Agr. Bull. 6, 1913.

7 U. S. Bur. Solls Circ. 65, pp. 13, 14, 1912. 
N. J.; the well-known onion farms of the Wallkill River Valley, Orange County, N. Y.; and the Oak Orchard lands north of Batavia, N. Y., are reclaimed peat deposits. Thousands of acres of land in these States now under intensive cultivation are underlain by peat: from 10 to 15 feet deep. Large areas of peat land in the Dismal Swamp district of Virginia and North Carolina are successfully devoted to the growth of potatoes, corn, wheat, and other crops. An average yield of more than 30 bushels of wheat per acre was obtained for several years on the well-tilled farm of the Wallace brothers, of Wallaceton, Va. Extensive use of peat soils for crop cultivation is also made in Minnesota, Wisconsin, Michigan, Iowa, Illinois, Indiana, and Ohio. Thousands of acres are already under cultivation, and millions more may be reclaimed by proper drainage and clearing. In fact, the economic future of entire counties in some of these States seems to be directly dependent upon the successful reclamation of peat bogs and swamps.

In Roseau, Marshall, Pennington, and Red Lake counties, Minn., where excellent crops of flax, barley, and oats are grown upon reclaimed areas, the peat is so shallow in many places that it may be penetrated by a deep tiller. Peat lands in the big tamarack and spruce swamps of northern Minnesota are being divided into homesteads with the design of draining and clearing them for agricultural use.

\section{TREATMENT.}

The prime requisite for the successful cultivation of peat land is a proper system of drainage. The water table must be kept far enough below the surface to protect the plants from excessive moisture, yet not so far that they will lack sufficient moisture. As pointed out by Alway, ${ }^{8}$ the climate controls to a large extent the depth to which the water table must be lowered. It is hardly possible to lower the water level too far in regions of heavy rainfall, but it is possible in regions of scant or medium rainfall. To obtain the greatest crops in parts of the country that have a cool climate the water table should be kept from 20 to 40 inches below the surface. The drainage problem is an engineering one, and before any attempt is made to reclaim a peat swamp or bog levels should be carefully taken to ascertain whether cheap drainage is feasible.

The drainage of most bogs is comparatively simple, but peatforming basins in which the peat is accumulating below a permanent water level can not generally be drained far below the surface of the peat except at great expense. However, many peat-forming basins in the region of the Great Lakes and in the New England

\footnotetext{
${ }^{8}$ Alway, F. J., Limitations on the cultivation of peat land in Minnesota: Am. Peat Soc. Jour., Apr., 1916, p. 65.
} 
States, where most of the depressions in which peat has accumulated were formed by the Wisconsin or last glacial drift, may be drained by short drainage canals that connect the edge of the basin at the lowest level with an adjacent stream. Tile drains, though expensive to install, generally prove more satisfactory and ultimately more economical in reclaiming peat areas than open ditches, which must frequently be dredged.

Many peat bogs must be cleared and grubbed before they can be cultivated. The surface of the bogs may generally be prepared for cultivation with comparative ease, but swamps that are overgrown with trees can not be cleared and grubbed except at great expense. However, this expense is sometimes offset by the value of the timber obtained.

The method of tillage is largely regulated by the depth of the peat. Shallow peat may be tilled in the same manner as ordinary loam, but the great peat deposits of northern Minnesota, Wisconsin, and Michigan, which range in depth from 5 to 20 feet, must be tilled in a radically different way.

In peat areas where summer frosts occur the soil must be specially treated. Damage to crops may usually be avoided by spreading a layer of sand, clay, or loam 2 or 3 inches thick, over the surface, but this is expensive and for large areas is therefore impracticable. Grass, clover, and certain other forage crops that are immune from damage by summer frosts may be sown on peat areas without risk of loss, but as climatic conditions differ in different areas crop practices suitable to one area may be unsuited to another.

Many peat soils, especially deep peat deposits, must be fertilized before they can be used for growing crops successfully, for they do not contain certain minerals that are required by plants. Potash must be added to many peats, and phosphates and nitrates must be added to some, though most well-decomposed peat soils, after they have been thoroughly plowed and aerated, are rich in nitrogen. If a peat soil is to be used to raise alkaline-soil crops and its acidity can not be corrected by plowing and aeration, lime should be applied to it. If properly used, lime will increase the fertility of the soil. Sources of limestone from which lime may be made are noted on pages 82-91. The limestone should be ground or burned before it is used as fertilizer, for the lime is thus made available immediately, whereas if used unburned some varieties of limestone, notably shell "marl," are relatively inactive during the first growing season after their application to the soil. Lime releases potash from certain silicates; it promotes the decomposition of the peat, freeing soluble nitrogen; if applied to sour soil, it corrects the acidity; and if applied to peat soils that harden in lumps on drying, it makes them easy to work. 
STOCK FEED.

Black humified peat is used both in Europe and in the United States for compounding stock feed. The peat is prepared in sub: stantially the same way as for fertilizer. (See pp. 61-62.) After: being air-dried and partly carbonized the peat is screened and re, duced to a powder containing about 10 per cent of moisture. The powdered peat is then used as an absorbent for the uncrystallized residues obtained in refining beet and cane sugar, which, because of their viscidity, are otherwise not suitable for feeding stock. This valuable food may thus be economically fed to cattle and other live stock without causing gastric disorders. It is said that the peat also stimulates digestion, contributes proteid substance, and is an excellent substitute for charcoal. Charred dried peat is also used as an in. gredient of food for poultry and other stock. In European coun, tries peat mull prepared from moss and sedge peat is used as a base for stock feed.

\section{ABSORBENT AND DISINFECTANT.}

Fibrous peat may be profitably employed as stable litter, for when so used it absorbs nitrogenous liquids, which, though valuable for use in fertilizers, are ordinarily wasted. Moreover, it checks decomposition and absorbs gases, so that it should be an effective deodorizer and disinfectant. For this use it is superior to lime and ashes and some of the more expensive disinfectants, and it is a nearly ideal material for use in earth closets and other receptacles for moist wasto organic matter. The best material for this purpose is found in the upper layer of deposits formed from sphagnum moss, an enormous quantity of which is available in the sphagnum bogs of the Great Lakes region and the New England States.

\section{FUEL.}

\section{GENERAL FEATURES.}

Because of its high content of carbon and because it will ignite and burn freely when dry, making a hot fire, peat is used as fuel in countries where the coal supply is below the normal requirements. In Europe between 15,000,000 and 20,000,000 tons of hand-cut and machine peat are consumed annually. Peat has been the only domestic fuel of the common people in Ireland from the traditional time when the country was deforested. The peat fire on the hearth, like the jaunting car, typifies Irish environment, and when the tourist: seeks a memento of his visit to that country he usually selects some souvenir carved from the black oak that has lain for centuries protected by beds of peat from the attacks of fungi and bacteria. Peat 
is cut by hand in blocks by the peasants for domestic use, and machine-cut peat is sold in blocks for both domestic and industrial use. (See Pl. IV, B.) Many attempts have been made both in Europe and in the United States to manufacture peat briquets for commercial use, but, though briquetted peat is more efficient than hand-cut or machine-cut peat, its manufacture has never advanced beyond the experimental stage on account of the high cost of production.

The peat in an undrained bog contains about 90 per cent of water, which must be reduced to 30 per cent before it can be used as fuel. By thoroughly draining the deposit about 10 per cent of the original water contained in the peat may be removed, but the remainder, which is largely held in the microscopic plant cells and minute intercellular spaces, can not be reduced below 70 per cent without drying in the open air or in a heated chamber. Artificial drying to remove all the excess moisture, however, requires the expenditure of so much heat in comparison with the heat obtainable from the fuel prepared by this method that it has not proved commercially feasible. Peat fuel should therefore be prepared during the air-drying season, which in the United States usually begins in April or May and ends in October. As Director Haanel ${ }^{9}$ has so well put it: "The forces of nature, the sun and the wind, which cost nothing, should be used, and any improvement in this process will lie in the direction of labor-saving devices." It seems, however, that artificial drying might be economically employed by utilizing waste heat from blast furnaces or other sources.

\section{. PEAT FUEL IN THE UNITED STATES.}

Although between $15,000,000$ and 20,000,000 tons of peat fuel are produced and consumed annually in Europe in generating heat and power, only small quantities of peat fuel have been produced in the United States because of the abundance of coal, which is more efficient and in normal times can be cheaply prepared and more readily transported to the consumer. The interest shown in peat fuel and its possibilities in the United States has thus far been largely scientific and experimental, and the attempts that have been made here to produce it on a large scale have not been successful. The failure, however, appears to have been due not to a lack of market for the product but to the lack of sufficient capital, to the inexperience of operators, to the failure to recognize that peat is inferior to coal, and to preventable engineering errors. Operators say that air-dried machine peat can be produced in the United States at a cost ranging

' Haanel, E., Peat as a source of fuel : Comm. Conservation, Canada, Ninth Ann. Rept., p. 16 (reprint), 1918. 
from $\$ 1.50$ to $\$ 5$ a ton, the exact figure depending on the size ani efficiency of the plant; and in some parts of the country it coul perhaps successfully compete with other fuels for both domestic ani industrial use. In many places where peat fuel has been used it this country it has proved very satisfactory and has found ready sale as fast as produced.

In recent years the increasing cost of producing coal has led tt a general advance in price. This condition, aggravated by an appre ciable reduction in the visible coal and oil supply and the rapid ex haustion of our forests, has strongly impressed upon economists an others the necessity of investigating other fuels and sources of powe: wherever they can be more economically used.

Van Hise, ${ }^{10}$ in urging the conservation of our wood and coal re. serves, said:

So far as practicable other products should be substituted for wood. The . original forests of the United States contained not less than $850,000,000$ acres, having not less than $4,800,000,000,000$ feet of merchantable saw timber. This was our magnificent original heritage. The United States as a Nation $h_{\text {il. }}$ existed a century and a quarter, and what have we now? In that brief time ap. proximately one-half of the value of our forests has gone.

So far as practicable substitutes should be used for coal. Even if all possible economies and substitutes are introduced, the most sanguine can not hope that the supply of fuels will be sufficient to meet the needs of the people for more than a small fraction of the time we look forward to as the life of this Nation.

Although peat fuel may not be extensively produced in the United States in normal times as long as there is an abundant supply of coal, except possibly in localities where conditions are peculiarly favorable, it has great potential value as a source of heat and power and may be utilized to conserve our reserves of coal and wood and also, during economic and industrial crises, may be used locally in some States to prevent a shortage of fuel.

Many extravagant claims concerning the fuel value of peat have been made, but the sooner its inferiority to coal is recognized the better for the peat-fuel industry. Over a million dollars have been spent in this country in trying to produce some form of peat fuel equal to coal in heating value, and yet we have no commercial peatfuel industry.

Careful consideration of all the factors leads to the conclusion that peat can be converted into fuel on a large scale in the United States only in the form of machine blocks, powder, and gas. Cut peat, however, is well adapted for home use by the owners of small de.

${ }^{10}$ Van Hise, C. R., The conservation of natural resources in the United States, pp. 210, 256, 359, New York, Macmillan Co., 1910. 
posits, but probably could not be marketed commercially. As com. plete descriptions of peat-fuel machinery and manufacturing processes may be found in United States Bureau of Mines Bulletin 16, only brief discussions of these phases of peat-fuel production are given here.

\section{METHODS OF PREPARATION.}

CUT PEAT.

Owners of small bogs who desire to prepare peat fuel for home use should adopt the handcut process, which is widely used in Ireland. Before this method can be employed the deposit must be thoroughly drained and cleared and the turf removed from its surface. Builtup bogs can usually be drained to the bottom by a simple system of surface ditches. Filled basins, however, can not generally be drained far below the surface of the peat without incurring great expense and are therefore not so well adapted to hand digging as built-up beds.

After the surface of the bog has been cleared the peat is dug in brick form with a special tool called a slane. (See Pl. IV, B.) This instrument, which can be made by a blacksmith, is a narrow spade with a sharp steel lug welded on one side at right angles to the edge of the blade.

The blocks of peat range from 8 to 10 inches in length, from 4 to 7 inches in width, and from 3 to 6 inches in thickness, their dimensions depending on the size of the slane. As soon as they are dug they should be removed to the drying grounds and stood on end or placed on covered racks. At the end of about four weeks, during which time they should be frequently turned, their moisture content will usually be reduced to about 30 per cent, and the blocks will then be ready for storage. As cut peat absorbs water rapidly, great care should always be taken to protect the dry blocks from rainfall. Peat fuel prepared in this way is bulky, easily crushed, and burns rapidly with considerable waste. In heating value it is superior to wood, but it is unfit for commercial use.

\section{MACHINE PEAT,}

If it is desired to produce peat fuel of better quality and in larger quantities than is possible by hand, the machine method should be adopted. This process, so far as the writer knows, is the only one that has proved commercially successful in Europe. The machinery for a small plant is simple and easily operated. It consists essentially of an excavator and a macerator. The purpose of the macerator is to grind the constituents of the wet peat into a homogeneous pasty 
mass, which may be shaped into compact blocks. In principle and form the latest types of peat machines are similar to the pug mill or grinding machine for plastic clay. Many of the experimental plants in the United States have used brickmaker's pug mills very slightly changed to grind peat and have found them well suited for the purpose. After being thoroughly macerated the peat is shaped into compact blocks as it comes from the machine or is spread in a layer from 8 to 12 inches thick on the drying grounds, and the bricks are marked off by hand as the spreading proceeds. When partly $d_{y} y$ the bricks are loosely stacked or placed on drying racks and thereafter handled in the same manner as cut peat.

A specially designed and constructed machine is used for the commercial production of peat fuel. This machine consists of a receiving hopper attached to a vertical or horizontal cast-iron body, in which revolve one or two knife-armed shafts. These shafts are also provided with spirally arranged flanges for moving the peat to the grinding knives and advancing it to the device for cutting the peat pulp into bricks of uniform length as it issues from the orifice of the machine. The principal types of peat machines of modern construction are fully described and illustrated in a publication of the Canada Department of Mines. ${ }^{11}$

Machine peat which is allowed to dry slowly contracts into a dense mass covered by a gelatinous, skinlike substance called hydrocellulose. After the moisture has been reduced to about 25 per cent this coating renders the machine peat impervious to water, even when immersed. It is clean to handle and burns freely, yielding an intense heat and producing no soot or other objectionable deposit. For open grates, this fuel is nearly ideal, and it is said that peat may be burned in the same stoves as coal and wood. However, the best results for household use could probably be obtained by burning it in a stove with relatively small grate openings and a restricted draft.

The following data, ${ }^{12}$ which are based upon the early experiments of the Canada Department of Mines, show the cost of a machine-peat fuel plant and of producing air-dried machine peat in commercial quantity in Canada in 1914. The cost of production was calculated from an output in five months of 3,300 short tons of fuel containing 25 per cent moisture.

\footnotetext{
11 Nystrom, Erik, Peat and lignite; their manufacture and uses in Europe: Canada Dept. Mines, Mines Branch, 1908.

${ }^{22}$ Haanel, B. F., Peat, lignite, and coal : Canada Dept. Mines, Mines Branch, Pub. 299, p. 27, 1914.
} 
Cost of a 30-ton Anrep peat plant and deposit.

100 acres of peat land at $\$ 6$ an acre

Peat machine, locomobile, tracks, cars, cables, etc

Blacksmith shop, office, loading platform, and railway siding -

Drainage, clearing, etc

Drainage, clearing, etc-1, $\frac{1,500}{10,700}$

Cost of producing a ton of air-dried machine peat.

Labor and supervision

$\$ 1.21$

Repairs, fuel, oil, waste, etc

.15

Amortization and interest.

.34

1. 70

Another experiment is now in progress in Canada, and it is understood that the cost of production is lower than that shown by these figures.

POWDERED PEAT.

For certain commercial uses powdered peat has many advantages over machine peat. The cheapest way to prepare it is by the airdrying process, by which the moisture may be reduced in the field. If raw peat is allowed to lie in heaps until natural drainage and evaporation have reduced the moisture content to about 50 per cent, it may be prepared for use under steam boilers by driving off about half the remaining moisture with waste heat from flues or other sources and pulverizing the resulting material. The powdered peat may then be blown with compressed air into the furnace, where, by means of forced draft, ignition is almost instantaneous, and instead of burning on the grate the peat forms a gas which gives a uniform fire throughout the combustion chamber. Good peat thus treated, when burned in furnaces designed to give the most complete and efficient combustion, will generate nearly as much energy in the form of live steam as the same weight of powdered coal. According to reports in this country powdered peat has great possibilities, not only for boiler firing but for metallurgical work and for use in cement and other kinds of kilns in which powdered coal has been successfully burned.

\section{PEAT BRIQUETS.}

Peat briquets are usually made from peat which has been air dried to a moisture content of 40 per cent. After the peat has been macerated and powdered, the moisture is further reduced to about 15 per cent by artificial drying. A binder is then mixed with the peat, and the mixture is compressed into cylindrical or prismatic shape by means $91065^{\circ}-22-6$ 
of a piston subjected to a pressure of 18,000 to 30,000 pounds per square inch.

Although there is little more heating value per pound in peat bri. quets than in machine peat, the briquets are cleaner, more compact, greater in heating value per volume, and generally more attractivo than machine peat. These advantages, however, are more than offset by the high cost of production.

Artificial drying requires so much heat in comparison with that obtainable from peat briquets prepared in this manner, and the cost of binders and compressing is so great that the process is at present of doubtful commercial value in the United States.

PEAT COKE AND CHARCOAL.

Peat charcoal was made in Europe for hundreds of years by piling cut peat in dome-shaped heaps, covering it with soil, and burning the peat with a restricted supply of oxygen. This process is little used now, and peat charcoal has been displaced by peat coke, which is manufactured in Europe by the Ziegler process. According to this method the coke is produced by heating machine peat blocks in specially designed retorts. Good peat coke is equal in quality to the best grades of charcoal produced from wood. If made from blocks of well-macerated, thoroughly decomposed peat that contains littlo ash, peat coke is compact and hard and should be able to compete with coke manufactured from coal. Alcohol, acetic acid, ammonia, ammonium sulphate, paraffin wax, illuminating and lubricating oil, phenol, and asphalt are some of the valuable by-products that may be obtained in coking peat by the dry distillation process.

Peat coke is extensively used in Europe, but in the United States, though it could probably be prepared for about $\$ 4$ a ton, this material has never been commercially produced. There are large deposits of peat suitable for the production of coke within a few miles of the iron-mining districts of Minnesota, and it seems that the practicability of locating peat-coke plants in this region should receive thoughtful consideration from engineers.

PRODUCER GAS AND BY-PRODUCTS.

There are two kinds of by-product gas-producing plants that use peat-the Mond and the Frank-Caro. The same principle is applied in both, and the differences between them are minor. Crushed peat is fed into a furnace in which combustion is regulated by steam and hot air. The peat burns at the bottom of the feed shaft and reacts upon the steam, forming water gas and ammonia. These gases are next cleansed of tar by means of a scrubber and are subjected to a fine shower of sulphuric acid, which converts the ammonia into am- 
monium sulphate and purifies the water gas. After being cooled the water gas may be used under steam boilers, in internal-combustion engines, and for other purposes.

Peat containing 2.5 per cent of combined nitrogen, when treated by the Mond process, at Cordigoro, Italy, is said to have yielded 170 pounds of ammonium sulphate to the ton. Gas-producing plants using peat fuel have been operated in England, Ireland, Germany, Sweden, Italy, and Russia, but not in the United States, although experiments have been made here. Analyses of the peats of the United States show that they are rich in combined nitrogen, from 70 to 85 per cent of which-a proportion that in some peats amounts to more than 2 per cent of their dry weight-could be recovered in the form of ammonium sulphate in by-product gas-producing plants.

PEAT AND PEAT MOSS AS ANTISEPTICS AND MEDICAMENTS.

ANTISEPTIC DRESBING.

Large quantities of peat or sphagnum moss have been produced and utilized in this country for surgical dressings. According to J. W. Hotson, ${ }^{13}$ under whose direction peat moss was collected in the Northwestern States for surgical use, 595,540 peat-moss pads were prepared from October, 1917, to November 11, 1918, by the northwestern and Atlantic divisions of the American Red Cross. Most of the moss was gathered by volunteer labor from the bogs of Washington, Oregon, and Maine, and the pads were used in military hospitals both at home and abroad. At first the surgeons of the United States Army were not disposed to use the pads, but later the value of sphagnum was universally recognized. The gathering of moss and preparing of pads was largely curtailed when the armistice was signed, but it seems that the value of peat moss as an absorbent and disinfectant demonstrated during the war will not be forgotten, and that it should be able to compete commercially with cotton for these purposes in normal times. A brief discussion of the best kinds of peat moss and of the method of gathering it is given in the report by Hotson. ${ }^{13}$

\section{MUD BATHS.}

At certain health resorts in Germany and Austria peat has long been successfully used for so-called mud baths, and during recent years experiments have been made to test the healing properties of peat in some of the sanitariums of this country. Well-decomposed peat, free from coarse or woody material, is the basis of the mixtures used, and it is reported that persons suffering from rheumatism and like diseases are materially benefited by the treatment.

${ }^{13}$ Hotaon, J. W., Sphagnum from bog to bandages: Washington Univ. Puget Sound Biol. Sta. Pub., vol. 2, No. 47, pp. 213, 243, 1919. 
OTHER USES.

\section{PACKING MATERIAL.}

Because of its lightness, resiliency, and antiseptic properties, peat makes excellent packing material and has long been used for packing eggs, fruit, vegetables, and fragile articles. Dry peat is a noncon. ductor of heat, and hence is valuable in ice houses and as a packing for water pipes. The best material is obtained from the fibrous types of peat, consisting of partly disintegrated grasses and mosses. Peat moss is also utilized in shipping flowers, shrubs, and plants.

PAPER, WOOD, AND CLOTH.

Peat was used a few years ago at a plant near Capac, Mich., for the manufacture of cardboard and paper. Grass and sedge peat would probably make the best paper stock, as this peat consists of strong fibrous material. Rugs are also made in this country from so-called wire grass (Carex stricta) grown upon peat. Peat is successfully used in Europe for the manufacture of wall board and other artificial wood products. German and Swedish manufac. turers have also made a durable yarn from a mixture of peat moss, shoddy, and wool, from which a cloth resembling cheviot is woven.

THE PEAT INDUSTRY IN THE UNITED STATES.

\section{PRODUCTION.}

The following tables show the production, prices, and value of the peat marketed in the United States in recent years:

Peat produced in the United States, 1916-1921.

\begin{tabular}{|c|c|c|c|c|}
\hline Year. & $\begin{array}{l}\text { Number } \\
\text { of plants } \\
\text { reporting. }\end{array}$ & $\begin{array}{l}\text { Quantity } \\
\text { (short } \\
\text { tons). }\end{array}$ & Value. & $\begin{array}{l}\text { Average } \\
\text { price per } \\
\text { ton. }\end{array}$ \\
\hline 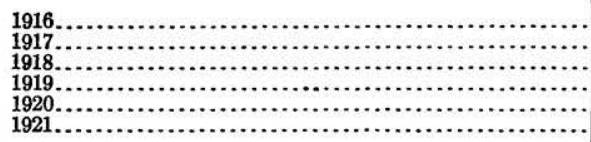 & $\begin{array}{l}13 \\
18 \\
25 \\
15 \\
18 \\
21\end{array}$ & $\begin{array}{r}52,506 \\
97,363 \\
107,261 \\
69,197 \\
73,204 \\
30,406\end{array}$ & $\begin{array}{r}\$ 369,104 \\
709,900 \\
1,047,243 \\
705,532 \\
921,732 \\
260,119\end{array}$ & $\begin{array}{r}97.0 \\
7.2 \\
9.7 \\
10.2 \\
12.5 \\
8.5\end{array}$ \\
\hline
\end{tabular}

Peat used in manufacturing fertilizer in the United States, 1916-1921.

\begin{tabular}{|c|c|c|c|}
\hline Year. & $\begin{array}{l}\text { Quantity } \\
\text { (shorty } \\
\text { tons). }\end{array}$ & Value. & $\begin{array}{l}\text { Average } \\
\text { price per } \\
\text { ton. }\end{array}$ \\
\hline $\begin{array}{l}1916 \\
1917 \ldots \ldots \ldots \ldots\end{array}$ & $\begin{array}{l}48,106 \\
92,263 \\
79,573 \\
54,690 \\
63,272 \\
29,460\end{array}$ & $\begin{array}{r}\$ 336,004 \\
658,500 \\
775,313 \\
557,240 \\
773,635 \\
251,046\end{array}$ & $\begin{array}{r}96.28 \\
7.14 \\
9.74 \\
10.10 \\
12.2 \\
8.58\end{array}$ \\
\hline
\end{tabular}


Peat used in manufacturing stock feed in the United States, 1916-1921.

\begin{tabular}{|c|c|c|c|}
\hline Year. & $\begin{array}{l}\text { Quantity } \\
\text { (short } \\
\text { tons). }\end{array}$ & Value. & $\begin{array}{c}\text { Average } \\
\text { price per } \\
\text { ton. }\end{array}$ \\
\hline 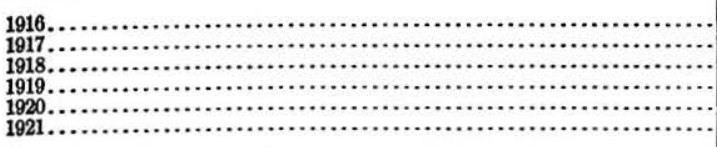 & $\begin{array}{r}4,300 \\
5,100 \\
7,096 \\
6,402 \\
9,102 \\
6946\end{array}$ & $\begin{array}{r}\$ 32,250 \\
51,400 \\
106,935 \\
98,940 \\
a 143,047 \\
b 9,073\end{array}$ & $\begin{array}{r}\$ 7.50 \\
10.08 \\
15.07 \\
15.45 \\
15.58 \\
9.59\end{array}$ \\
\hline
\end{tabular}

a Includes small quantity of moss and stable litter.

$\checkmark$ Includes small quantity of fuel, moss, and stable litter.

\section{IMPORTS AND EXPORTS.}

The imports of peat in 1920 consisted of peat moss or litter, which is used largely for packing material. The quantity was 2,762 tons, or six times as much as in 1919 . The price per ton, however, fell from $\$ 35$ to $\$ 13$. The imports in 1921 amounted to 3,450 tons, but the price per ton fell to $\$ 6.60$. No exports of crude or peat products were reported in 1920 or 1921.

SUMMARY.

Peat and pcat moss used in the manufacture of peat products in the United States in 1920 and 1921.

\begin{tabular}{|c|c|c|c|c|c|c|}
\hline \multirow[b]{2}{*}{ Kind of product. } & \multicolumn{2}{|c|}{ Production. } & \multicolumn{2}{|c|}{ Imports. } & \multicolumn{2}{|c|}{ Consumption. } \\
\hline & $\begin{array}{l}\text { Quantity } \\
\text { (short } \\
\text { tons). }\end{array}$ & Value. & $\begin{array}{c}\text { Quantity } \\
\text { (short } \\
\text { tons). }\end{array}$ & Value. & $\begin{array}{c}\text { Quantity } \\
\text { (short } \\
\text { tons). }\end{array}$ & Value. \\
\hline 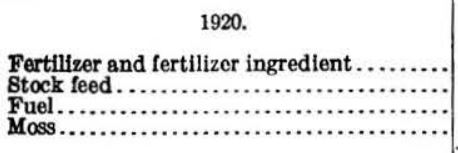 & $\begin{array}{r}63,272 \\
a 9,182 \\
750 \\
(b) \\
\end{array}$ & $\begin{array}{c}\$ 773,635 \\
a 143,047 \\
5,0.50 \\
\text { (b) }\end{array}$ & $\begin{array}{r}2,762 \\
\cdots\end{array}$ & $\$ 36,20 \mathrm{i}$ & $\begin{array}{r}63,272 \\
9,182 \\
750 \\
2,762 \\
\end{array}$ & $\begin{array}{r}\$ 773,635 \\
143,047 \\
5,050 \\
36,201 \\
\end{array}$ \\
\hline & 73,204 & 921,732 & 2,762 & 36,201 & 75,966 & 957,933 \\
\hline 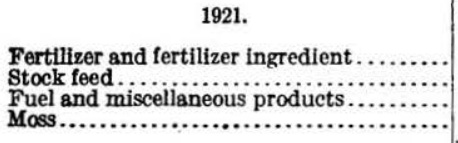 & $\begin{array}{c}29,460 \\
c 946 \\
(c) \\
(c) \\
\end{array}$ & $\begin{array}{c}251,046 \\
c 9,073 \\
(c) \\
(c) \\
\end{array}$ & 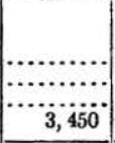 & ㄴ............. & $\begin{array}{r}29,460 \\
c 946 \\
(c) \\
c 3,450 \\
\end{array}$ & $\begin{array}{r}251,046 \\
c 9,073 \\
(c) \\
c 22,754 \\
\end{array}$ \\
\hline & 30,406 & 260,119 & 3,450 & 22,754 & 33,856 & 282,873 \\
\hline
\end{tabular}

a Includes small quantity of moss and stable litter.

$b$ Included with stock feed.

$c$ Inludes small quantity of fuel, moss, and stable litter.

\section{CONSUMPTION.}

The consumption of peat and peat moss (production plus-imports) was 69,661 tons, valued at $\$ 721,877$, in $1919 ; 75,966$ tons, valued at $\$ 957,933$, in 1920 ; and 33,856 tons, valued at $\$ 282,873$, in 1921 .

\section{DISTRIBUTION OF PEAT PLANTS.}

The 21 plants reporting production in 1921 were distributed as follows: New Jersey 4, New York 4, California 3, Illinois 2, and 
Florida, Georgia, Massachusetts, Michigan, Minnesota, New Hamp. shire, North Carolina, and Wisconsin 1 each. California was the largest producer, with an output of 12,672 short tons, valued at $\$ 117,580$. New Jersey was second in rank, with an output of 12,051 tons, valued at $\$ 94,269$; and Illinois was third, but the State total can not be published without revealing confidential information, as there were only two producers.

\section{PRODUCERS OF PEAT IN THE UNITED STATES.}

The following individuals and companies reported to the Geo. logical Survey that they produced crude peat or peat products in the United States in 1921:

Alphano Humus Co., Whitehall Building, New York, N. Y.

Appleton Peat Products Co., Appleton, Wis.

Blaine, J. H., Hopewell Junction, N. Y.

Chapman, I. S., \& Co. (Inc.), 937 Third Street, San Bernardino, Calif.

Craig, William H., Fishkill, N. Y.

Day, James H., 35 South Street, Milford, N. H.

Hennepin Atomized Fuel Co., 406 Tribune Annex, Minneapolis, Minn.

Humus Natural Manure Co., 1964 Broadway, New York, N. Y.

Hyper-Humus Co., Newton, N. J.

International Products Co., 132 Boylston Street, Boston, Mass.

McElhone, Asa, Fishkill, N. Y.

Manito Chemical Co., Peoria, Ill.

Marcrum, J. G., Netcong, N. J.

National Humus \& Chemical Co., Chassell, Mich.

Pacific Humus Co., 205 Central Building, Pasadena, Calif.

Phos-Pho Germ Manufacturing Corporation, New Bern, N. C.

Ranson, Robert, St. Augustine, Fla.

Riverside Orange Co. (Ltd.), Arlington Heights, Riverside, Calif.

Sims, Alfred F., Sag Harbor, N. Y.

Southern Humus Co., Smyrna, Ga.

Wiedmer Chemical Co., Pierce Building, St. Louis, Mo.

COMMERCIAL FACTORS.

CAUSES OF FAILURE.

Although some of the elements of successful peat production have been mentioned, full descriptions of the factors that control the profitable utilization of a peat deposit are here given in order to show the causes of past failures. Some of the attempts made in this country to produce peat on a commercial scale have not been successful, but many of the failures appear to have been due not to a lack of market for the product but to the lack of sufficient capital, to the inexperience of operators, and to the injudicious choice of deposits, plant sites, manufacturing processes, and machinery. All these factors should be carefully considered by prospective producers.

\section{LOCATION.}

The location of the deposit is the prime factor in the successful operation of a peat plant. Many deposits containing peat suitable 
for commercial use are known in all the peat areas, but under present conditions in the United States only deposits located near the markets and where cheap labor and transportation may be obtained can be profitably developed. Thus the conditions for successfully producing peat fuel are most favorable in Minnesota, Wisconsin, Michigan, and the New England States, which contain but little coal and because of their cold climate and extensive manufacturing industries consume large quantities of fuel. In the Southern Atlantic States, although the climate is mild and the demand for fuel relatively light compared with that in the Northern States, peat is the only local mineral fuel available. (See Pl. I.)

Fertilizer plants should, of course, be preferably located near the agricultural districts and the commercial fertilizer plants, notably in New York, New Jersey, Illinois, and Virginia. As peat fertilizer is not well known in this country a market will have to be created in many places, but if high-grade products can be sold at a low price this should not be difficult.

\section{QUANTITY AND QUALITY.}

The prospective deposit should be carefully surveyed to insure that it contains a sufficient quantity of good peat before plans are made for the erection of a plant. Typical samples should be analyzed to ascertain whether the peat is of suitable quality for the purpose desired. One of the great handicaps suffered by the peat industry has been the lack of uniformly good quality, and as the success of a plant depends upon the kind of peat used due care should be observed in its selection.

\section{SIZE OF DEPOSIT.}

The size of the deposit to be selected depends upon the capacity of the machinery to be used, but it is generally unwise to erect a plant on a deposit less than 100 acres in area and 4 feet in depth. The average peat deposit will yield approximately 200 tons of air-dried peat per acre-foot. The quantity of peat in a given deposit may therefore be approximately determined by multiplying the area in acres by the depth in feet and the product by 200 . The topographic maps issued by the United States Geological Survey show many valuable peat deposits as swamps, the area of which may be readily estimated. The dimensions of deposits that have not been mapped should be carefully measured.

Although peat deposits less than 4 feet deep may be advantageously used for the growth of crops, they are of little value as a source of fertilizer, fuel, or other commercial peat products. Numerous tests of depth should therefore be made and the average thickness of the 
peat determined. An earth auger may be employed for this purpose, but a sampling tool especially designed by the late Prof. Davis ${ }^{15}$ for testing the depth of peat deposits was used in field work for this report and is superior to the earth auger for sampling peat.

\section{SELECTION OF SAMPLES.}

Samples of peat should typify the entire deposit from which they are taken or a definite portion of it. Specimens consisting of material taken at depth intervals of 1 foot and at areal intervals of 100 yards in a small, shallow deposit usually represent the general composition of the deposit. It is obviously impossible to obtain a typical sample of the peat in a large, deep deposit. Such a deposit should be divided into blocks and typical samples taken from each block. Tests are generally made at depth intervals of 2 feet and areal intervals of one-fourth of a mile in large deposits. The material taken from each block may later be mixed in order to obtain a representative specimen. Structural features should be noted as deposits are sampled, and if the peat is distinctly bedded samples should be taken from each bed and separately analyzed. If the lower strata contain a large proportion of inorganic impurities the fact should be noted in order that these strata may be avoided in excavation.

As analyses of dry peat show its chemical composition and value for fertilizer and fuel as well as analyses of wet peat, it is unnecessary to place samples taken for analysis in air-tight containers. For a simple test about 10 pounds of raw peat and for a detailed chemical analysis about 40 pounds are required. Samples should ordinarily be air-dried to a moisture content of about 50 per cent in the field and sent to the laboratory in canvas bags. However, if it is desired to identify the plant remains, small specimens should be placed in sealed bottles or air-tight soil cans, so that the peat may later be examined under the microscope in its native condition.

\section{SIMPLE TESTS.}

Black, thoroughly decomposed friable peat from a deposit that has been well drained, aerated, and successfully cultivated for several seasons, is usually suitable for an ingredient of commercial fertilizers or, when limed and inoculated with nitrifying organisms or composted with manure, for a direct fertilizer.

Some evidence of the fuel value of peat may be obtained from a small sample that has been macerated in the hands, pressed into a cake, and allowed to dry in the sun. If it is easily disintegrated and

${ }^{25}$ Davis, C. A., The uses of peat for fuel and other purposes: Bur. Mines Bull. 16, p. 71, 1911. 
plastic while wet, it may be readily worked in a peat machine, and if when dry the cake is dense, tough, and hard, the peat will probably make good fuel unless the ash content is too high. However, if the dry peat cake is soft, fibrous, and easily crushed, the peat is not well adapted to the manufacture of fuel.

The heating value and ash content of prospective peat fuel for home consumption may be determined by a simple practical test. A typical sample should be taken from the bog, thoroughly macerated, dried, and weighed. If when burned in an ordinary heating stove the heat generated is almost equal to that produced by ordinary bituminous coal, and if after complete combustion the weight of the accumulated ash does not exceed 20 per cent of the weight of the dry peat put into the stove, its usefulness as domestic fuel is established.

\section{PREPARATION OF SURFACE.}

The surface of peat deposits must be prepared by draining and clearing before the peat can be removed. As these factors have already been discussed, only a brief summary is here given. The water level should be reduced by a system of tile drains or ditches to a point between 3 and 5 feet below the surface. Only deposits that can be cheaply drained should be selected. Bogs are usually easy to drain, but the drainage of some peat-forming basins is so difficult that their surfaces can not be economically prepared for peat production by known methods. Marsh deposits and bogs are cleared by removing the living grasses and mosses and grubbing out the shrubbery. The clearing of swamps overgrown by mature trees is expensive, but this expense is sometimes offset by the value of the timber obtained.

PROCESSES AND MACHINERY.

The failure of many peat plants in the United States has been due to improper choice of manufacturing processes and machinery. The excess moisture in the peat must be eliminated at as early a stage as possible in the manufacturing process. This drying can be most economically done by evaporation on the surface of the deposit. Whether for the manufacture of fertilizer or fuel, machinery of proved efficiency must be selected. Field equipment propelled by caterpillar drives seems to be well suited for use on boggy surfaces. The most successful peat-fuel machinery that has been used in North America was designed by the Canada department of mines and operated at Alfred, Ontario. John N. Hoff, of New York City, has successfully operated peat-fertilizer machinery for several years on a deposit near Great Meadows, N. J. Though experimental work is always commendable if it is properly limited, the methods and 
machinery that are successful elsewhere in North America and in the peat-producing countries of Europe should be used. The peat in dustry of European countries is well developed, and detailed infor: mation concerning European peat machines is available in Bureat of Mines Bulletin 16 and in the report by Nystrom, published by the Canada Department of Mines. ${ }^{16}$

\section{MARKETS.}

The principal markets for fertilizer peat are in or near the great agricultural States, notably New York, New Jersey, Illinois, Virginia, Kentucky, Tennessee, and Florida, and the largest buyers are the manufacturers of commercial fertilizer, brokers in fertilizing materials, florists, and farmers. Markets could probably be created in many other agricultural States. Stock-feed peat is sold to manu-: facturers of stock feed and to live-stock growers. Practically all the peat deposits in this country occur in regions remote from coal fields, and it seems that markets for peat fuel might therefore be created in some of the States where peat is abundant.

\section{AREAS OF SPHAGNUM MOSS.}

Sphagnum moss grows in low, undrained regions where the humidity of the air is high and the temperature low and where other conditions of growth are favorable. These conditions are found in. the extreme northwestern and northeastern parts of the United States, as well as in the northern part of the Great Lakes States.

Northwestern States.-The largest areas of high-grade sphagnum moss in this country are in Washington and Oregon. It is estimated that there are 25,000 acres of bog land in the western part of Washington alone, much of which is overgrown by sphagnum moss suitable for surgical dressings and packing material. Some of this moss grows in climbing bogs, and in a few of these bogs the center is higher than the margin, a condition especially favorable for the growth of surgical sphagnum.

Northeastern States.-According to G. E. Nichols, ${ }^{17}$ the bogs of Maine contain more sphagnum than those of any other State in the North Atlantic region, but relatively large sphagnum bogs also occur in some of the other Northeastern States. Sphagnum moss suitable for surgical dressings, as well as for other purposes, grows in the following localities:

${ }^{16}$ Nystrom, Erik, Peat and lignite, their manufacture and uses in Europe: Canada Dept. of Mines, Mines Branch, 247 pp., 33 pls., 1908.

${ }^{17}$ Nichols, G. E., written communications, 1918. 
Maine :

Aroostook County : Otter Pond. Penobscot County: Alton Bogs. Waldo County : Cross Pond. Washington County: Lake Hadley, Lake Meddybemps.

New Hampshire:

Cheshire County: Bog near East Jaffrey.

Hillsborough County: Bogs near Greenfield, South Milford, and Ponemah.

Rockingham County: Bog near Fremont.
Vermont:

Addison County: Bristol Pond, East Monkton Bog, Monkton Bogs.

Grand Isle County: Isle La Motte Bog.

Franklin County: Cranberry "Marsh."

Massachusetts :

Middlesex County: Littleton Bog, Tophet " Swamp," West Bedford Marsh.

Norfolk County: Norwood Bog, Walpole Bog.

Many other areas in the Northeastern States are overgrown by sphagnum, but most of it is not suitable for surgical dressings. However, it could be advantageously used for packing material and stable litter. Further references to areas in the New England States that contain sphagnum are made in the discussions of the localities tested for peat.

Other States.-Although large areas of sphagnum are found in the Great Lakes States, varieties suitable for surgical dressings are not abundant because of the excessive heat in summer and the extreme frost in winter. The largest sphagnum bogs in this region occur in Beltrami, Itasca, Koochiching, and St. Louis counties, Minn.; in the northern counties of Wisconsin, Michigan, and Indiana; in northern and western New York; in Portage County, Ohio; and in Pennsylvania. Immense quantities of moss that might be used for packing material, paper stock, and stable litter could be obtained from these bogs. In New York surgical sphagnum grows in the bogs of the Adirondack region in the northern part of the State; in the Cicero Swamp, north of Syracuse; at Mud Pond, near Fairville; and in the western part of the State. Small quantities of surgical sphagnum grow in a few bogs in Pennsylvania, but according to Jennings Sphagnum papillosum, one of the best species, does not occur there. The Pymatuning Swamp near Hartstown, Lost Pond, Long Pond, and Half Moon Lake contain sphagnum, but it is not well adapted for surgical use. The best species probably occur in the highlands of the north-central and western parts of the State. A list of the areas in western Pennsylvania that contain sphagnum may be found in a report by Jennings. ${ }^{18}$ Areas covering many square miles in Camden County, N. C., are said to contain Sphagnum. cymbifolium, one of the highest grades of peat moss.

Further data showing the specific location of sphagnum in the different States are given in the discussions of localities where the

${ }^{18}$ Jennings, O. E., A manual of the mosses of western Pennsylvania, Pittsburgh, Pa., 1913. 
peat was sampled. The large quantity of sphagnum peat in the domestic deposits shows that sphagnum moss grew more extensively in this country in former times than it does now. It seems that when many sphagnum bogs matured and uncongenial trees and other plants appeared the sphagnum could not survive and that it. died out in other bogs when the tamarack was cut.

\section{MARL AND OTHER LIMESTONE DEPOSITS ASSO- CIA'TED WITH BEDS OF PEAT.}

\section{GENERAL CONDITIONS.}

Many peat deposits in the northern or glacial region are underlain by beds of marl, a white or gray claylike variety of limestone composed largely of calcium carbonate $\left(\mathrm{CaCO}_{3}\right)$, which was brought into lakes and marshes by springs or stream waters and precipitated through the agency of blue-green algae and stoneworts (Chara). These plants extract carbon dioxide from the water and thus cause the deposition of the lime held in solution. Deposits of fresh-water marl are common in Wisconsin, in the southern peninsula of Michigan, in northern Indiana, Illinois, and Ohio, in western New York, and in northwestern Pennsylvania. Shell limestone deposited by organisms is another kind of calcareous material which commonly occurs beneath peat in the coastal region. Dense fine-grained limestone, marble, travertine, oolitic limestone, and chalk, though not generally associated stratigraphically with peat, commonly occur in regions near peat deposits. On account of its acidity much peat land is of little value for general farming when first cleared, but it could be economically treated with lime from associated or near-by deposits and made to yield large alkaline or neutral-soil crops. Brief reference to the treatment of limestone for agricultural use and to the effect of lime on peat soils is made on page 65. Data concerning beds of both fresh-water and marine marl that occur in association with peat are given in conjunction with the discussions of peat localities on pages $91-202$.

The following extract from a paper by Burchard and Emley ${ }^{19}$ shows the age and general location of the principal limestone formations in the States that contain the most valuable peat deposits.

\section{DISTRIBUTION OF LIMESTONE BY STATES.}

NEW ENGLAND STATES.

Practically all the limestone in the New England States is so highly crystalline as to be considered marble. The principal areas

19 Burchard, E. F., and Emley, W. E., The source, manufacture, and use of lime: U. \$. Geol. Survey Mineral Resources, 1913, pt. 2, pp. 1530-1546, 1914. 
of calcareous rocks are near the coast of Maine, in Knox and Waldo counties, and in western Vermont, Massachusetts, and Connecticut. Lime is the principal product of the area in Knox County, Maine, and it is made also in Berkshire County, Mass., and in Litchfield and Fairfield counties, Conn. In Vermont also the marble is suitable for the manufacture of lime, although the production of lime in that State falls below that of the other States mentioned.

\section{NEW YORK, NEW JERSEY, AND PENNSYLVANIA.}

New York and Pennsylvania are both especially rich in limestone. A belt of Silurian limestones, extends eastward from Buffalo nearly across New York, and adjoining this belt on the south are Devonian formations, which also carry limestones. On account of the proximity of these limestone areas to several east-west railroads and of the exceptional purity of the stone, they are extensively quarried, especially for flux for blast furnaces at Buffalo. East of Lake Ontario, in addition to a lobe of Silurian limestone there is an area of Ordovician rock which contains limestone in the vicinity of Watertown. Along St. Lawrence River and the northern end of Lake Champlain, Cambrian and Lower Ordovician limestones occur, and quarries are operated in the vicinity of Ogdensburg and Plattsburg. Lime is burned at many of these localities in New York, but the lime industry is much more widely distributed in Pennsylvania than in New York. In Pennsylvania limestones of commercial value occur in the western half of the State in the Carboniferous areas, and chiefly in the Pennsylvanian ("Coal Measures") formations. From the middle of the State eastward the limestones are chiefly of late Cambrian, Ordovician, Silurian, and Devonian ages, and the limestone areas are distributed in long, narrow strips, analogous to the outcrops of the "valley" limestones in the southern Appalachians. The dips of the beds in eastern Pennsylvania are steep as compared with those in the western half of the State.

Many of the limekilns in Pennsylvania are small and are operated intermittently by the farmers to furnish lime for local fertilizers.

Much of the limestone of New York, especially in the western half of the ${ }^{\bullet}$ State, is high in calcium carbonate; in Pennsylvania both high-calcium and high-magnesian stone occurs in abundance, the magnesian rock being especially well represented in the valleys of southeastern Pennsylvania. The "cement" rock and purer limestone beds of the Lehigh district in eastern Pennsylvania are quarried to some extent for crushed stone. Near Philadelphia the limestone has been subjected to intense folding and pressure, and in many places the beds have been recrystallized to marble, although they are utilized mostly for lime rather than for marble. In northwest New 
Jersey the beds utilized for crushed stone in the Lehigh district of Pennsylvania extend into Warren, Sussex, and Hunterdon counties, N. J. Much of this rock is highly magnesian.

The New Jersey stone area is limited to the northwestern and northern parts of the State, for the deposits of the Coastal Plain, which underlie the remainder of the State, consist mainly of feebly consolidated materials, such as clay and sand.

MARYLAND, DFLAWARE, VIRGINIA, WEST VIRGINIA, NORTH CAROLINA, AND SOUTH CAROLINA.

The limestones of this area fall physically into three groups: (1) The nonmetamorphosed limestones of the Appalachian valleys; (2) the metamorphosed or crystalline limestones of the Piedmont region, both of which are hard limestones; and (3) the soft limestone of the Coastal Plain. The great limestone deposits of the valley of Virginia are the most noteworthy in this area. They are easily accessible and consequently are extensively utilized for lime burning. These same limestone belts extend from northern Virginia across the corner of West Virginia and through Maryland into Pennsylvania and Delaware. West of the Alleghenies there are deposits in eastern West Virginia, but only in the northern part of the State are they extensively utilized for the production of stone and lime. -Southwestward they extend into Tennessee. Limestone is not abundant in the Piedmont region, except where it has been altered to marble, but it is quarried in a few places, notably in Henderson, Transylvania, and Yadkin counties, N. C.; Cherokee County, S. C.; Loudoun County, Va.; and Frederick and Carroll counties, Md. The hard limestones may be either high-calcium or magnesian rocks. The soft limestones of the Coastal Plain consist largely of shell marl, and important quarries are situated in Norfolk County, Va.; Craven and Jones counties, N. C.; and Charleston County, S. C. The shell limestones are generally quite pure and contain little magnesia, but they contain considerable silica in places, owing to the admixture of sand.

TENNESSEE, MISSISSIPPI, ALABAMA, GEORGIA, AND FLORIDA.

Calcareous rocks predominate, at least so far as suitability for quarrying is concerned, in Tennessee, northern Alabama, and northern Georgia, and much of the limestone is suitable for the manufacture of lime. In Franklin County, Ala., there are extensive quarries in an oolitic limestone that strongly resembles the famous Bedford (Ind.) limestone, and is of about the same geologic age. Much of the limestone quarried in these States is crushed for concrete, railroad ballast, wagon roads, and for fluxing iron ores. Some 
hard limestone occurs in Tishomingo County, Miss., but owing to the proximity of large quarries in Alabama it has not yet been utilized to an appreciable extent. In the deposits of the Coastal Plain there are soft limestones of Cretaceous and Tertiary age. In Choctaw, Clarke, and Washington counties, Ala., these rocks are utilized. Florida is largely underlain by beds of shell marl and chalky limestone of Tertiary and Recent age, but these limestones are covered, for the most part, by comparatively thick deposits of sand and gravel. The Coastal Plain material that is utilized in this State for limestone and lime is mainly in the vicinity of Ocala.

\section{WISOONSIN AND MICHIGAN.}

A considerable part of the area of Wisconsin and Michigan is thickly covered by glacial deposits of clay, gravel, and sand. Doubtless this cover retards the development of quarries to some extent, first by obscuring outcrops of good limestone and second by the expense which its removal entails in quarry operations. Silurian rocks underlie the greater part of the eastern tier of countis in Wisconsin and contain a thick formation of magnesian limestone. This rock is quarried in many places for stone to be burned into lime and also to be crushed for concrete, macadam, and railroad ballast. The proximity of some of the quarries in eastern Wisconsin to water transportation on Lake Michigan has greatly aided their rapid development. The Silurian rocks on the west and extending across the southern tier of counties are bordered by a belt of rocks of Middle and Upper Ordovician age, which also contain magnesian limestone. The limestone quarries west of Green Bay and west and south of Lake Winnebago, as well as those in the southern counties, are in these rocks. Between the border of the Middle Ordovician area and the crystalline area of north-central Wisconsin is a wide belt of rocks of late Cambrian and early Ordovician age, consisting chiefly of alternating beds of sandstone and magnesian limestone. There are a few limestone quarries in these rocks, mainly near Mississippi River between Prairie du Chien and Hudson. The greater part of the lime made in Wisconsin is burned from Silurian magnesian limestone in the eastern part of the State, but there are a few small kilns burning lime for local use in the southern and western areas. One thin formation of relatively high calcium Ordovician limestone is burned for lime in Lafayette County.

In Michigan Devonian formations, consisting largely of limestone, border the northern part of the southern peninsula of the State along Lakes Michigan and Huron and also cross the southeastern part of the State from Lake St. Clair to the Ohio line. A small area of Silurian limestone borders Lake Erie in the extreme southeast corner 
of the State, and at the extreme northern point of the southern penin. sula there is also a narrow strip of Silurian rocks. Bordering the north end of Lake Michigan and the Strait of Mackinac are Silurian formations that constitute the country rock for some distance back from the shore in the northern peninsula. This is an extension of the belt of rocks that borders Lake Michigan in eastern Wisconsin. Adjoining the Silurian area on the west and north are rocks of Mid. dle and Upper Ordovician age that enter the State from west of Green Bay, Wis.; and succeeding these rocks toward the west and north are formations of late Cambrian and Ordovician age that form the shore line of Lake Superior east of Marquette and part of the shore line between Marquette and Keweenaw Point, but which con. tain little limestone of importance. The central area of the southern peninsula of Michigan is underlain by rocks of Carboniferous age, largely sandstone and shale. The limestone quarries in Michigan are therefore distributed mainly around the borders of the southern peninsula and near the shores of Green Bay and Lake Michigan in the northern peninsula, and are mainly in rocks of Ordovician, Si. lurian, and Devonian age. There are certain exceptions, however, such as the quarries near Saginaw Bay, which are in Mississippian limestone. The limestones of the Carboniferous and of the Devonian are generally high-calcium rocks; the lower limestones are generally high in magnesium.

ILLINOIS, INDIANA, OHIO, AND KENTUCKY.

The central part of Illinois is so heavily drift laden that quarries can not generally be opened economically, and this condition applies also to much of the area of northern and central Indiana and to parts of Ohio.

In this group of States the principal limestone formations are of Ordovician, Silurian, Devonian, and Carboniferous age. Of the Carboniferous rocks the lowest or Mississippian series contains the most important limestone formations in the western part of the area. In Illinois the Ordovician formations in the north-central part, the Silurian in the Chicago area, and the Silurian and Devonian in the northwestern part of the State are all worked for limestone. Some highcalcium limestone is found in the Ordovician, but the beds are mainly magnesian. The Silurian limestones are also magnesian, but the Devonian limestone carries a high percentage of calcium carbonate. Along Mississippi River and the lower Illinois Valley practically all the limestone quarries are in Mississippian rocks, which are generally well exposed in the bluffs, and consequently economical quarrying sites are not difficult to find. Thus far development has taken place principally at points where railroads happen to be built near good limestone outcrops, although, of course, some quarries have been 
opened at a distance and connected with the main line by spur tracks. On Ohio River in southern Illinois Mississippian rocks are also quarried. In eastern Illinois a few quarries have been opened in Pennsylvanian rock, notably a large quarry producing limestone for blast furnace flux and Portland cement in Vermilion County. The Carboniferous rocks generally are fairly high in calcium and low in magnesium, but the Mississippian rocks particularly are apt to contain a considerable percentage of silica, in some places in the form of chert. Lime is made from all these limestones in Illinois.

Indiana is underlain by formations having the same range in age as those in Illinois. The Pennsylvanian rocks outcrop in western Indiana; Mississippian rocks are found in a belt a little west of the middle of the State and in an area in the extreme northeast corner; Devonian and Silurian formations underlie the northwestern, central, and northeastern portions, with Ordovician rocks in the southeast corner, which is affected by the Cincinnati anticline. The limestone quarries in Indiana are chiefly in the Mississippian, Devonian, and Silurian formations. Thirty-eight counties are reported as producers of limestone. Lawrence and Monroe counties are the principal producers of building stone in the State. The famous oolitic limestone quarried about Bedford as a center occurs in these counties in a favorable situation for quarrying. This limestone is of Mississippian age and has few counterparts in the United States so far as its physical characteristics are concerned. This Bedford stone is of medium fine-grained, even texture, and is composed of small round concretionary grains and of broken fragments of fossils, all compactly cemented together by calcite. The stone occurs in masses 30 to 50 feet and even 70 feet thick.

In addition to the counties mentioned Owen, Crawford, Harrison, and Washington counties, Ind., are said to be underlain by this oolitic limestone. In Bartholomew, Decatur, Jennings, and Ripley counties there is considerable quarrying in limestone of Devonian and Silurian age, and farther north, in Delaware, Grant, Wabash, and Huntington counties there are many quarries. This latter area is mainly in magnesian limestone of Silurian age. Some lime is burned from the Silurian limestone in Indiana, but the greater part of the output comes from the high-calcium Mississippian oolitic rock.

In Ohio the limestone quarries are chiefly in three areas which correspond to three geologic systems of rocks. In the northwestern and western parts of the State the quarries are very largely in Silurian magnesian limestone; in southwestern Ohio near Cincinnati rocks of Ordovician age yield limestone low in magnesia but rather high in silica, or in silica and alumina; in eastern Ohio, in Carroll, Stark, 
Tuscarawas, and other counties, Pennsylvanian limestones an quarried. These latter rocks are high in calcium carbonate. Lim is burned principally from the magnesian Silurian limestone and from the high-calcium Pennsylvanian beds.

Kentucky is underlain by the same geologic formations that have been mentioned above in Illinois, Indiana, and Ohio. In fact, these four States are more closely associated geologically than any other group of the United States having an equal area. Kentucky has a less area, however, of Silurian rocks, these formations being confined to a narrow strip in central Kentucky around the border of Ordovician rocks that form the Cincinnati anticline. The forms. tions that yield the greater part of the limestone quarried in Kentucky are of Ordovician and Mississippian age. The Ordovician system is represented in the central part of the State southward from Cincin. nati, Ohio, and quarries in it are operated in Harrison, Bourbon, Scott, Franklin, Anderson, Fayette, Jessamine, Mercer, Boyle, and other counties. A small quantity of lime is burned in this area Limestone quarries in Mississippian rocks are scattered pretty well through the southern part of the State, but the most important area is near Bowling Green, Warren County. An oolitic limestone outcrops here, very similar in physical characteristics and of nearly equivalent age to the stone described above occurring in the Bedford (Ind.) district. This oolitic limestone is in a massive, homogeneous stratum 22 feet thick. Other areas of oolitic stone occur near Somer. set, Pulaski County, and in Barren, Simpson, Logan, Meade, Hardin, Grayson, Caldwell, Todd, Christian, Wolfe, Powell, and Rockcastle counties and is quarried in a few of them. This oolitic stone has a high content of calcium carbonate and is burned for lime at Bowling Green and several other places.

\section{MINNESOTA.}

The limestone areas are mainly in the southeastern part of Min. nesota along Mississippi River and westward to the middle of the southern part of the State.

A considerable part of Minnesota is thickly covered with glacial deposits of clay, gravel, and sand. Probably this cover retards the development of quarries to some extent, first, by obscuring outcrops of good rock, and, second, by the expense which its removal entails in quarry operations. The majority of the stone quarries are, therefore, near the streams, where erosion has cut sections through the glacial drift and exposed the hard rock, generally in terraces or bluffs.

Much of the limestone quarried along Mississippi River and its tributaries in Houston, Winona, Wabasha, Goodhue, and Washing. 
ton counties is of Cambrian and Lower Ordovician age. The beds are generally highly magnesian and received the name "Magnesian" limestones in early geological reports. They are blue when freshly mined, but weather to a buff color. They lie horizontal and in beds from a few inches to a foot or two in thickness. This belt of rock extends up Mississippi River nearly to St. Cloud and up Minnesota River beyond Mankato; it is quarried extensively near Kasota and Mankato both for building stone and for natural cement and at other places for crushed stone and for the manufacture of lime. Along Mississippi River the limestone is used extensively for riprap.

Overlying the Cambrian and Lower Ordovician rocks in southeastern Minnesota, between Mississippi and St. Peter rivers, are rocks of Middle and Upper Ordovician age, including the so-called "Trenton" limestone. Quarries have been opened in these beds near Rochester, Mantorville, Faribault, and Minneapolis. The color and bedding of these rocks are similar to those of the earlier Ordovician rocks. Near Faribault certain of the limestone beds consist of so nearly pure calcium carbonate that the stone has been burned for the manufacture of lime for use in beet-sugar refining. Most of the material for structural purposes produced from the so-called "Trenton" limestone is used in the form of rubble and crushed rock.

The local surface rock at Minneapolis is the so-called "Trenton" limestone. This rock consists of beds of high-calcium, fine-grained, dense light-gray rock, beds of bluish to greenish argillaceous magnesian limestone, and beds that approach shale in texture. The firstmentioned beds are the most desirable for all purposes, but most of the quarries are obliged to move considerable of the inferior stone, and more or less of it is worked into the product.

Lime is burned from local stone in the following Minnesotat counties: Blue Earth, Fillmore, Goodhue, Mower, Rice, and Scott. As noted above, the limestone in most of these counties is magnesian, but that in Rice and Mower counties is high in calcium carbonate. Analyses of certain of these limestones and limes are given in Mineral Resources for 1911, Part II, pages 673 and 701. The magnesian stone is of Ordovician age, earlier than the "Trenton," and the high-calcium stone belongs to the so-called "Trenton" and later formations. The quarry at Le Roy, Mower County, is in an area of Devonian rock.

Limestone is quarried in 16 counties in Minnesota, 6 of which produce lime; and in addition lime is burned near Duluth from stone quarried in Ohio and shipped by boat through the Lakes. As at present developed there are more quarries of limestone in Minnesota than of any other rock, and if the value of the output of lime, as well as of limestone, is considered, the industry based on limestone 
quarrying yields more wealth than any other phase of the quarrying industry.

IOWA.

Although a considerable part of the surface of Iowa is covered with glacial drift, streams have so dissected the drift mantle as to produce many good exposures of the underlying beds of hard rock. Paleozoic rocks underlie the eastern two-thirds as well as the southwest corner of the State. Cretaceous rocks underlie the remaining northwestern portion, with the exception of a few square miles in the northwest corner, which are underlain by Huronian (Sioux) quartzite. The Paleozoic rocks, which begin with the Cambrian and Lower Ordovician along Mississippi River, in the northeast corner of the State, outcrop in successive northwestsoutheast belts and include rocks of Middle and Upper Ordovician, Silurian, Devonian, and Carboniferous age. The Carboniferous rocks include large areas of Mississippian and Pennsylvanian rocks, and a very small area of probably Permian beds in Webster County. The Cambrian and Ordovician systems comprise beds of magnesian limestone, soft, friable sandstone, and some fairly high calcium limestone; the Silurian and Devonian systems carry magnesian limestone and high-calcium limestone, respectively; the Mississippian series is composed mostly of high-calcium limestone, but some magnesian and some cherty beds and some sandstone; and the Pennsylvanian series contains much sandstone and a smaller proportion of shaly limestone. Most of the systems contain shale, but no account is taken of that material in this connection.

The distribution of stone quarries follows closely that of the streams and incidentally that of the railroads, and also falls almost entirely within the area of Paleozoic rocks. With possibly the exception of limestone quarries in Montgomery County, apparently there are no regularly working quarries in the Cretaceous areas, although doubtless there are a few local pits that produce limited quantities of Cretaceous limestone for use on the farms.

The limestone quarries of Iowa are distributed over the eastern two-thirds of the State and have been opened in each of the Paleozoic limestones mentioned above. Probably the greater number of quarries are in limestone of Mississippian age, although there are numerous quarries in the Cambrian and Ordovician areas. There are about 243 limestone quarries in Iowa in 42 counties. Twenty-three of these quarries in 7 counties produce stone for making lime. High-calcium lime is burned from formations of Mississippian and Devonian age, and magnesian lime is produced from Mississippian and Ordovician rocks. 
LOUISIANA.

Practically the whole of Louisiana is within the Coastal Plain, and is underlain by formations of Tertiary and Quaternary age, consisting principally of clay, sand, and soft limestone. Locally there are hard beds of limestone and sandstone in the Tertiary deposits, and if situated conveniently to transportation lines they may be quarried.

The only limestone reported as quarried in Louisiana is near Winnfeld, Winn Parish. Hand specimens of this limestone sent to the Survey are of a dense, subcrystalline, bluish limestone, gashed and seamed with white calcite veins from the thickness of a knife blade up to three-fourths of an inch. The material makes a good crushed stone for ballast, macadam, and concrete. An analysis published in Mineral Resources for 1911 shows nearly 92 per cent calcium carbonate, and the material is probably suitable for the manufacture of lime.

\section{DISTRIBUTION OF PEAT DEPOSITS AND QUANTITY OF PEAT AVAILABLE.}

\section{GENERAI FEATURES.}

The most valuable peat deposits of this country may be roughly assigned to two general regions-the northern or glacial, and the Atlantic coastal-and this division, though partly geographic, indicates the chief differences in origin and in the quality of the peat. The northern peat region, which contains the most extensive deposits in the United States, includes Minnesota, Wisconsin, Michigan, eastern South Dakota, the northern parts of Iowa, Illinois, Indiana, and Pennsylvania, and New York, New Jersey, and the New England States. (See Pl. I.) This region is characterized by numerous ponds, marshes, and lakes formed by glacial action during Pleistocene time and by relatively low temperature and high humidity in the growing season. Most of the peat originated in basins. Probably the largest peat deposit in this country is in the northern region. It covers nearly 4,000 square miles, or about 2,500,000 acres, in Beltrami, Koochiching, Roseau, and Marshall counties, Minn. The Great Lakes States contain a larger quantity of high-grade workable peat than all other States. New England has many climbing bogs and much sphagnum peat, and the most extensive deposits occur in Maine and Massachusetts. It is estimated that in Maine alone there are deposits which would yield $100,000,000$ short tons of air-dried peat, $48,000,000$ tons of which are readily accessible. New England peat is of good quality.

The Atlantic coastal region, which contains many workable deposits, embraces the southern part of Delaware, the eastern parts of 
Maryland, Virginia, North Carolina, South Carolina, and Georgia, and all of Florida. The nearness of the ocean causes heavy rain. fall and high relative humidity in this region, and the deposits occur in drowned valleys and lagoons, which were formed by the gradual subsidence of the Coastal Plain and by wave action, and on flat, im. perfectly drained areas farther inland. This region is characterized by many salt and fresh water marshes and swamps, in which the de. posits have been formed largely by trees, sedges, and marsh grasses, some of the grasses tolerating salt water around their roots. The largest deposits of good peat in this region are in Virginia, North Carolina, and Florida.

Although there are a few peat deposits along the coasts of the New England States and New Jersey that are related in origin and composition to those assigned to the Atlantic coastal region, by far the most numerous bogs of the Northeastern States are of the filled. basin kind, and these States are therefore classed in the northern region.

Peat also occurs in a narrow belt of land adjoining the Gulf Coast, in Siskiyou, Los Angeles, Orange, and San Bernardino counties, Calif., in the valleys of Sacramento and San Joaquin rivers, and in the basis of several lakes and rivers in Oregon and Washington.

In 1909 the total quantity of known peat in the United States, exclusive of Alaska, calculated as air-dried peat, was estimated to be $12,889,000,000$ short tons, ${ }^{21}$ but recent field investigations seem to justify an increase of this figure to $13,827,000,000$ tons, distributed as follows:

Approximate distribution of known peat in the United States, by Statcs (csti mated as air-dried peat).

\begin{tabular}{|c|c|c|}
\hline State. & $\begin{array}{c}\text { Area } \\
\text { (acres). }\end{array}$ & $\begin{array}{c}\text { Quantity } \\
\text { (short tons). }\end{array}$ \\
\hline 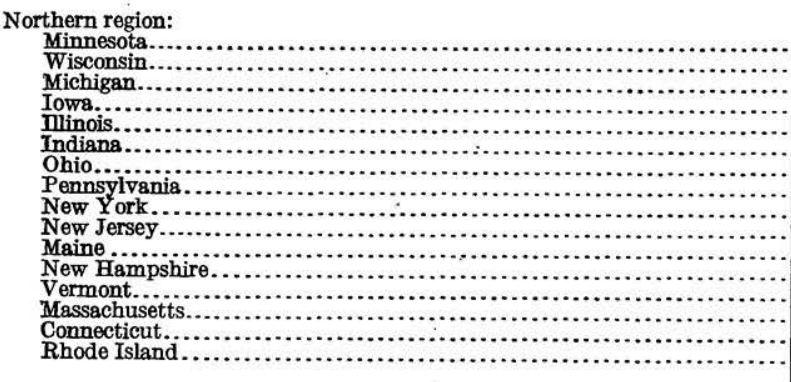 & 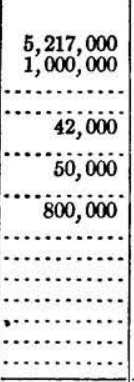 & $\begin{array}{r}6,835,000,000 \\
2,500,000,000 \\
1,000,000,000 \\
22,000,000 \\
10,000,000 \\
13,000,000 \\
50,000,000 \\
1,000,000 \\
480,000,000 \\
15,000,000 \\
100,000,000 \\
1,000,000 \\
8,000,000 \\
12,000,000 \\
2,000,000 \\
1,000,000\end{array}$ \\
\hline & n............. & $11,050,000,000$ \\
\hline
\end{tabular}

21 Davis, C. A., Peat resources of the United States exclusive of Alaska: U. S. Geol. Survey Bull. 394, pp. 65-66, 1909. 


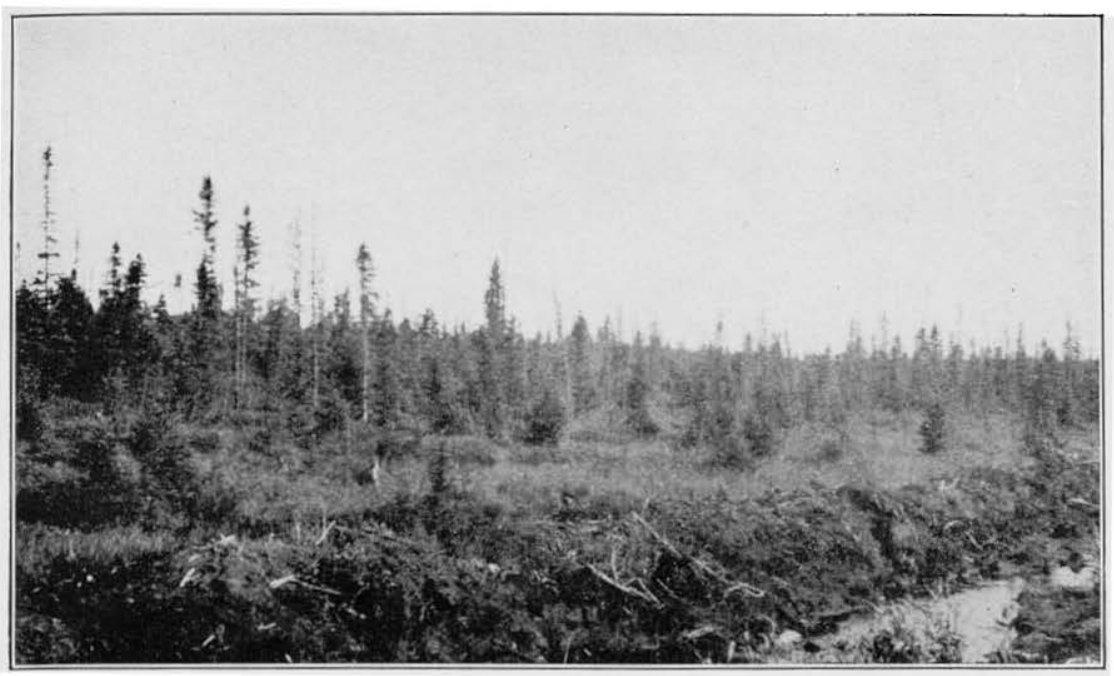

A. SPRUCE-SPHAGNUM BOG.

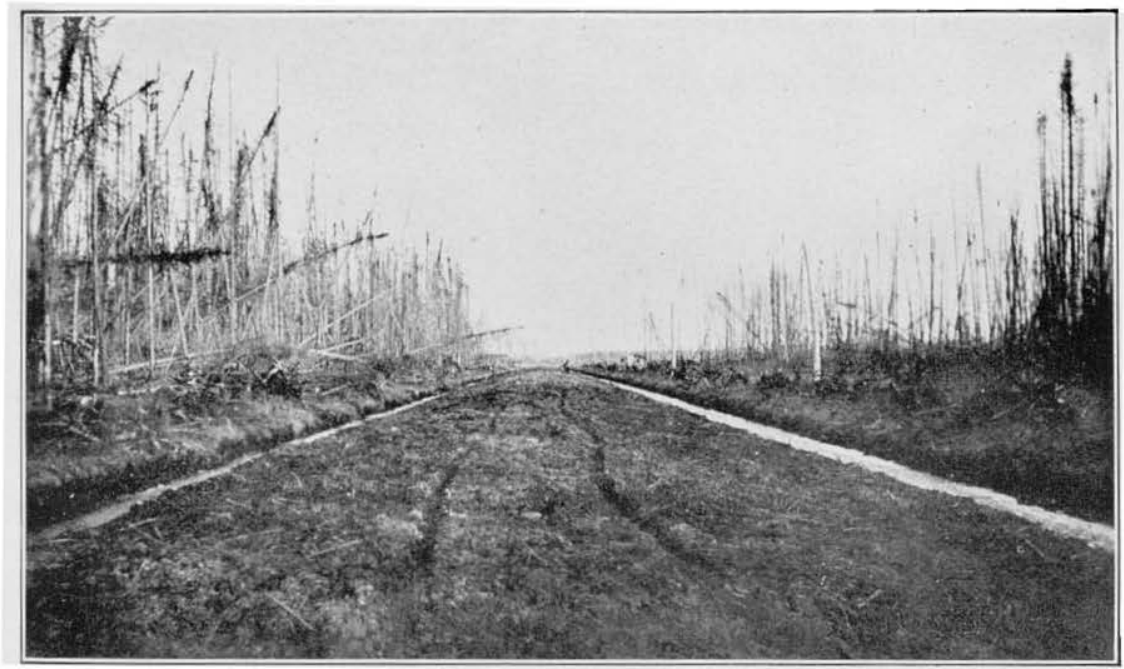

B. TAMARACK SWAMP.

Predominant Types of Peat Deposits in Northern Minnesota. 



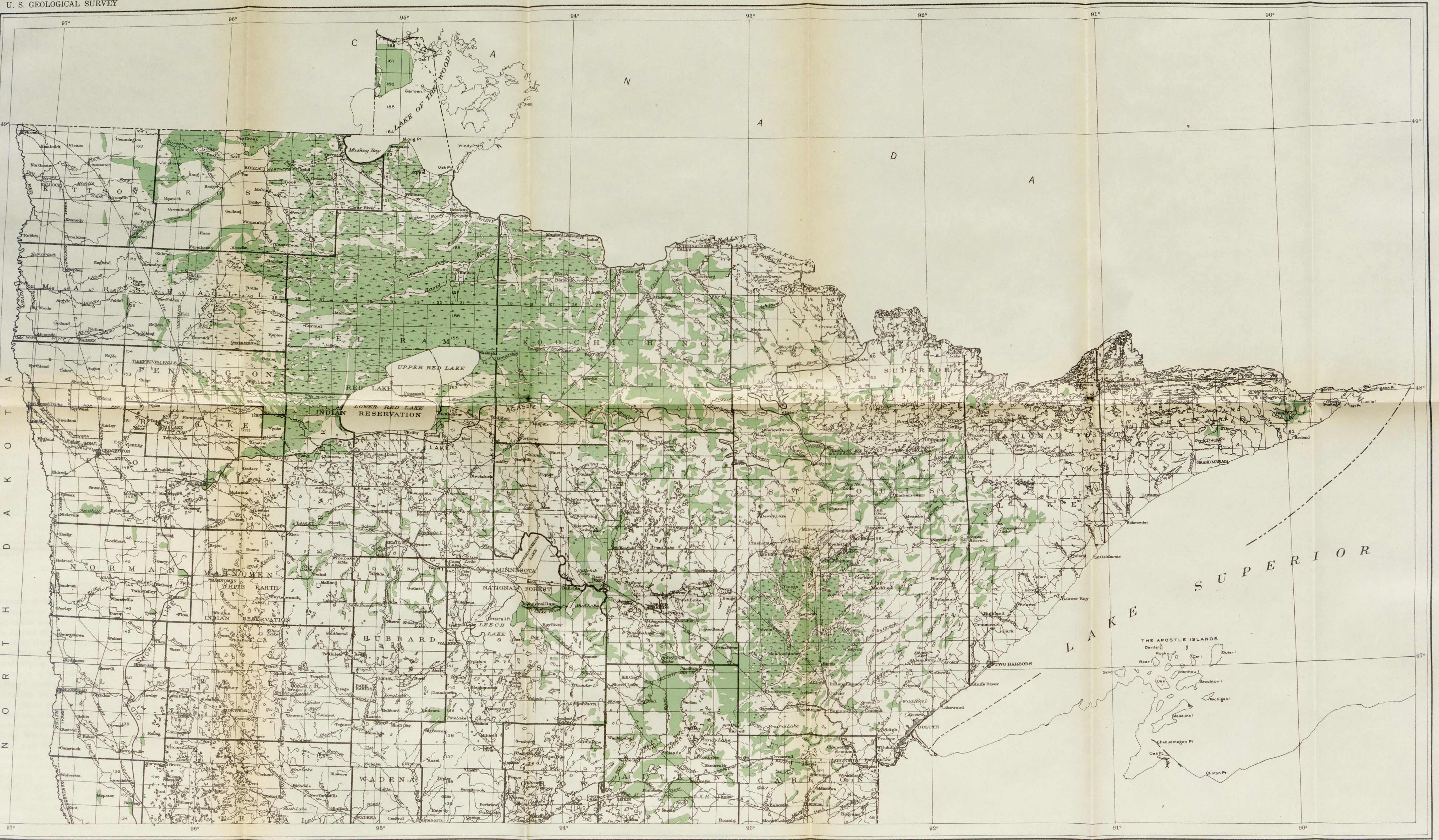
MAP SHOWING DISTRIBUTION OF PEAT IN NORTHERN MINNESOTA 

Approximate distribution of peat in the United States, by States-Continued.

\begin{tabular}{|c|c|c|}
\hline State. & $\begin{array}{c}\text { Area } \\
\text { (acres). }\end{array}$ & $\begin{array}{l}\text { Quantity } \\
\text { (short tons). }\end{array}$ \\
\hline 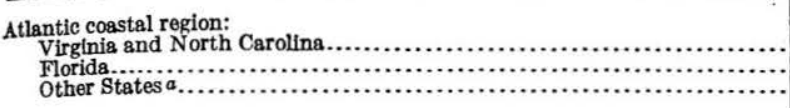 & 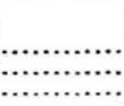 & $\begin{array}{r}700,000,000 \\
2,000,000,000 \\
2,000,000\end{array}$ \\
\hline . & n........... & $2,702,000,000$ \\
\hline 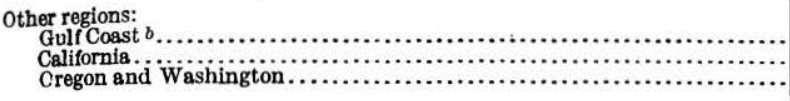 & $\because 80,000$ & $\begin{array}{r}2,000,000 \\
72,000,000 \\
1,000,000\end{array}$ \\
\hline & ….......... & $75,000,000$ \\
\hline Grand total................... & & $13,827,000,000$ \\
\hline
\end{tabular}

a Includes Delaware, Maryland, South Carolina, and Georgis.

$b$ Exclusive of Florids.

\section{NORTHERN REGION.}

MINNESOTA.

The peat deposits in Minnesota, which are the most extensive in the United States, occur in basins that are of glacial origin. The largest of these basins are in the area that was covered by the waters of glacial Lake Agassiz and of other temporary glacial lakes. Besides these there are many deposits in ponds, marshes, and swamps in the smaller basins in the glacial drift. Most of the large filled-basin deposits matured long ago and are covered with built-up peat. Most of the peat occurs in spruce and tamarack swamps, known locally by the Indian term " muskeg." (See Pl. V.) Cedar swamps are also common. Sphagnum moss, sedges, grasses, and heath shrubs are the most notable plants that grow in the open areas. The greater part of the sphagnum is unsuitable for surgical use, and good moss for this purpose was found only in the vicinity of Cohasset, Coleraine, Deer River, Warba, and Wawina, Itasca County; Littlefork, Margie, Nakoda, and Wisner, Koochiching County; and Elmer, Floodwood, Island, and Wallace, St. Louis County. The most extensive peat deposits lie in the northern part of the State; but some peat occurs in nearly every county except in the Red River district of western Minnesota and the Driftless Area in southeastern Minnesota. However, the deposits of workable extent may be roughly assigned to the three areas described below:

1. The northern area includes the "muskeg" swamps of Aitkin, Beltrami, Cass, Clearwater, Crow Wing, Itasca, Koochiching, Roseau, and St. Louis counties. (See Pl. VI.) This area contains more peat than any other of equal size in the United States. There are about 1,300,000 acres of peat land in Beltrami County alone. Koochi- 
ching and St. Louis counties each contain at least 1,000,000 acres 0 peat, most of which exceeds 6 feet in depth.

2. The central area embraces Anoka, Chisago, Douglas, Hennepin Isanti, Mille Lacs, Ramisey, Sherburne, Washington, and Wrigh counties. The deposits of Anoka County, within a few miles 0 Minneapolis and St. Paul, contain a large quantity of good peat.

3. The southern area includes Blue Earth, Carver, Dakota, Free born, Le Sueur, Nicollet, Rice, Scott, Steele, and Waseca counties The deposits in this area are relatively small and shallow.

It is estimated that the deposits of Minnesota are capable of yield. ing $6,835,000,000$ short tons of air-dried peat, the distribution of which, by counties, is shown in the following table : ${ }^{22}$

Area and depth of peat in Minnesota, and quantity available as air-dried peat. by counties.

\begin{tabular}{|c|c|c|c|}
\hline County. & $\begin{array}{c}\text { Area } \\
\text { (acres). }\end{array}$ & $\begin{array}{l}\text { A verage } \\
\text { thickmess. } \\
\text { (feet). }\end{array}$ & $\begin{array}{c}\text { Quantity } \\
\text { (short tons). }\end{array}$ \\
\hline 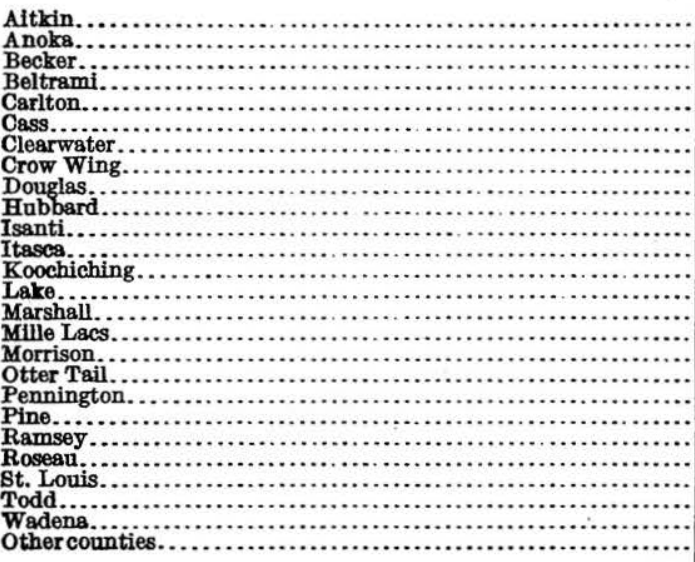 & $\begin{array}{r}397,300 \\
30,000 \\
12,800 \\
1,299,200 \\
35,000 \\
75,000 \\
128,000 \\
61,300 \\
5,000 \\
10,000 \\
10,000 \\
250,000 \\
1,000,000 \\
150,000 \\
50,000 \\
25,000 \\
10,000 \\
75,000 \\
10,000 \\
75,000 \\
1,500 \\
250,000 \\
1,192,000 \\
10,000 \\
5,000 \\
50,000\end{array}$ & $\begin{array}{r}6 \\
7 \\
5 \\
7 \\
10 \\
5 \\
5 \\
6 \\
6 \\
6 \\
5 \\
6 \\
7 \\
6 \\
5 \\
5 \\
5 \\
5 \\
5 \\
5 \\
5 \\
5 \\
7 \\
5 \\
5 \\
5\end{array}$ & 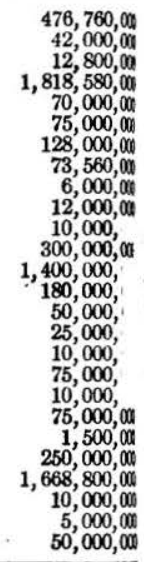 \\
\hline & $5,217,100$ & & $6,835,000,000$ \\
\hline
\end{tabular}

Information concerning the chemical composition of this peat is given in the tables of analyses (pp. 37-42). Nearly all the accessible peat deposits in Minnesota are described in detail in Bulletin 16 of the Minnesota Geological Survey.

\section{WISCONSIN.}

Most of the peat deposits of Wisconsin, like those of Minnesota, occupy depressions formed by glacial action during the Wisconsin stage of glaciation. Large spruce-sphagnum and tamarack-sphagnum

\footnotetext{
${ }^{22}$ Soper, E. K., The peat deposits of Minnesota : Minnesota Geol. Survey Bull. 16, p. 33,
} 1919. 


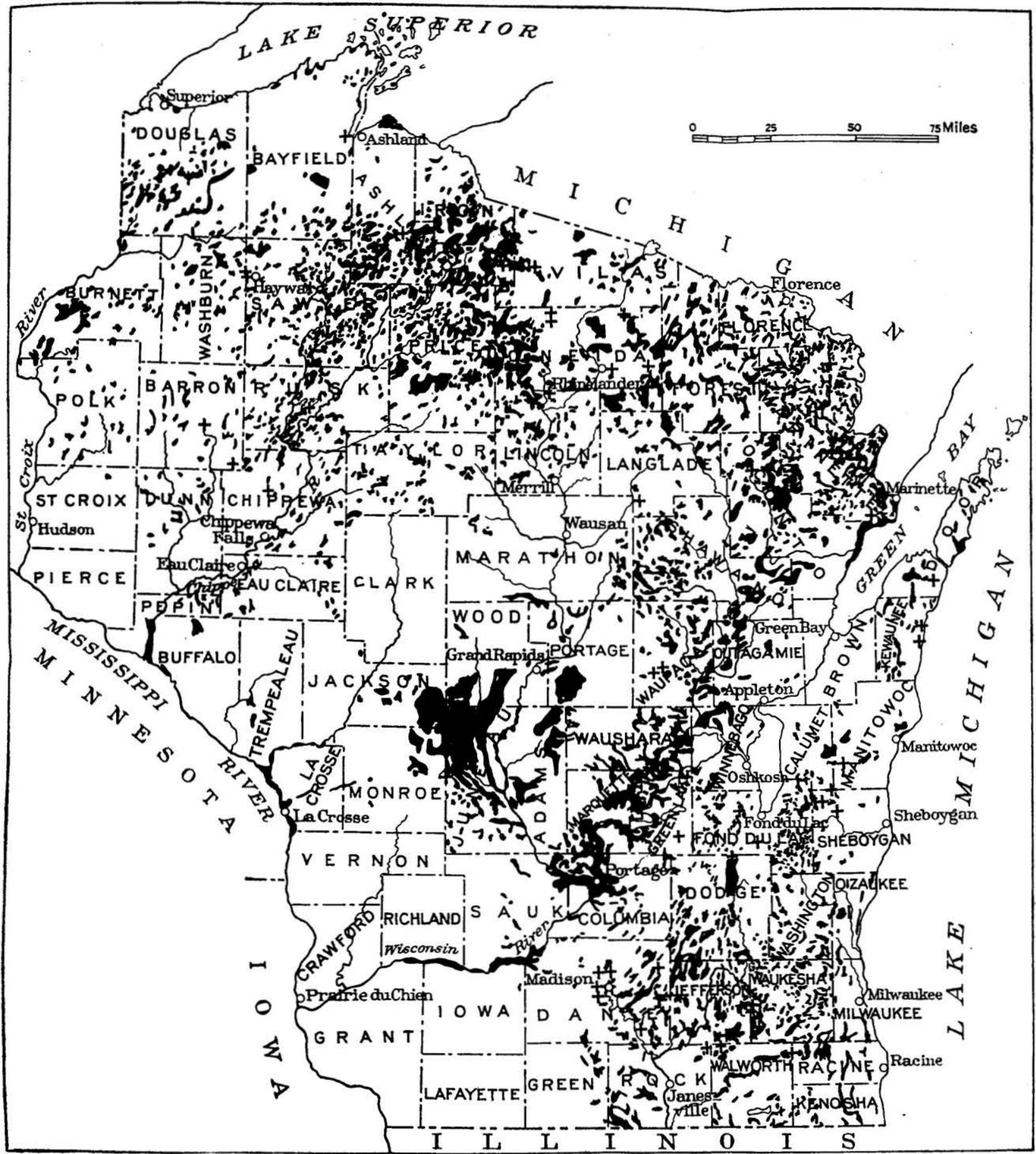

MAP OF WISCONSIN SHOWING DISTRIBUTION OF UNDRAINED LAND.

Peat deposits that have been tested are marked thus + . 

bogs and swamps predominate in the northern part of the State, and many grass-sedge meadows and marshes occur in the southern part. The deposits that have been tested range from 1 to 20 feet in depth and from 1-to 32,000 acres in area. Much peat in northern Wisconsin has been destroyed by fire, and the charred remains, deeply buried in some places beneath thick fibrous peat, indicate that the conflagration occurred thousands of years ago. Many areas have been only recently damaged by fire, and the underlying peat is covered with 2 or 3 feet of charred material. In some places the earlier vegetation has been destroyed, and entirely different plants have succeeded.

The general distribution of undrained land in Wisconsin is shown in Plate VII. Valuable peat deposits are found throughout the State except in the southwestern part. There is some peat in nearly all the undrained areas, but the most valuable deposits are in the following counties: Ashland, Dane, Dodge, Door, Florence, Fond du Lac, Green Lake, Jefferson, Juneau, Kewaunee, Langlade, Marinette, Marquette, Oconto, Oneida, Price, Rock, Sawyer, Shawano, Sheboygan, Vilas, Walworth, Waukesha, Waupaca, Waushara, Winnebago, and Wood. A detailed description of the areas that have been tested for peat is given in a State report. ${ }^{23}$

It is estimated that there are a little more than 1,000,000 acres of peat land in Wisconsin capable of yielding 2,500,000,000 short tons of air-dried peat. The following table ${ }^{24}$ shows the distribution, acreage, depth, and contents of 50 deposits that have been examined by the Wisconsin Geological and Natural History Survey. The approximate location of these deposits, which include a little more than one-tenth of the total area of peat in the State, is shown in Plate VII. The chemical composition of the peat is shown in the table of analyses (pp. 51-57).

Area, depth, and contents of typical peat deposits in Wisconsin.

\begin{tabular}{|c|c|c|c|c|c|}
\hline $\begin{array}{c}\text { Deposit } \\
\text { No. }\end{array}$ & Location (nearest town or city). & $\begin{array}{c}\text { Area } \\
\text { (acres). }\end{array}$ & $\begin{array}{l}\text { Depth } \\
\text { (feet). }\end{array}$ & Acre-feet. & $\begin{array}{l}\text { Quantity } \\
\text { of air-dried } \\
\text { peat (short } \\
\text { tons). }\end{array}$ \\
\hline $\begin{array}{r}1 \\
2 \\
3 \\
4 \\
5 \\
6 \\
7 \\
8 \\
9 \\
10 \\
11 \\
12\end{array}$ & 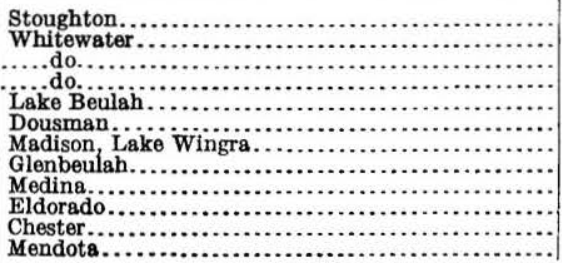 & $\begin{array}{r}520 \\
640 \\
200 \\
12,800 \\
500 \\
9,600 \\
100 \\
9,600 \\
6,400 \\
4,000 \\
32,000 \\
1,300\end{array}$ & $\begin{array}{r}9 \\
9 \\
7 \\
5 \\
12 \\
3 \\
2 \frac{1}{2} \\
9 \\
12 \\
8 \\
6 \\
10\end{array}$ & $\begin{array}{r}4,680 \\
5,760 \\
1,400 \\
64,000 \\
6,000 \\
28,800 \\
250 \\
86,400 \\
76,800 \\
32,000 \\
192,000 \\
13,000\end{array}$ & $\begin{array}{r}936,000 \\
1,152,000 \\
280,000 \\
12,800,000 \\
1,200,000 \\
5,760,000 \\
50,000 \\
17,280,000 \\
15,360,000 \\
6,400,000 \\
38,400,000 \\
2,600,000\end{array}$ \\
\hline
\end{tabular}

${ }^{23}$ Huels, F. W., The peat resources of Wisconsin : Wisconsin Geol. and Nat. Hist. Survey Bull. 45, pp. 82-144, Econ. Ser. 20, 1915.

24 Idem, p. 170. 
Area, depth, and contents of typical peat deposits in Wisconsin-Continued.

\begin{tabular}{|c|c|c|c|c|c|}
\hline $\begin{array}{l}\text { Deposit } \\
\text { No. }\end{array}$ & Location (nearest town or city). & $\begin{array}{c}\text { Area } \\
\text { (acres). }\end{array}$ & $\begin{array}{l}\text { Depth } \\
\text { (feet). }\end{array}$ & Acre-feet. & $\begin{array}{l}\text { Quantity } \\
\text { of air-dried } \\
\text { peat (short } \\
\text { tons). }\end{array}$ \\
\hline 13 & Markesan. & ............... & 12 & & \\
\hline $\begin{array}{l}14 \\
15\end{array}$ & $\begin{array}{l}\text { Marshall........ } \\
\text { Rhinelander.... }\end{array}$ & 1,200 & $\ddot{6}$ & 7,200 & $1,440,000$ \\
\hline 16 & Camp Douglas.. & 2,000 & 2 & 4,000 & 800,000 \\
\hline 17 & $\begin{array}{l}\text { Babcock } \\
\text { Mendota, Yahara River. }\end{array}$ & & & 33,500 & 700000 \\
\hline 302 & Madison, University Bay. & 100 & 6 & 600 & 120,000 \\
\hline 303 & Fond du Lac................ & 800 & 7 & 5,600 & $1,120,000$ \\
\hline 304 & Waupaca............... & $\begin{array}{r}1,500 \\
60\end{array}$ & $10+$ & $\begin{array}{r}15,000 \\
1,200\end{array}$ & $3,000,000$ \\
\hline $\begin{array}{l}305 \\
306\end{array}$ & iel, Manitowoc....... & 10,000 & & 80,000 & $16,000,000$ \\
\hline 307 & & & & & \\
\hline 308 & Kiel Shoboyg & $\dddot{5}, 500$ & $\begin{array}{l}6 \\
4\end{array}$ & 22.000 & \\
\hline 309 & Kiel, Sheboygar & 2,000 & $\frac{4}{3}$ & 6,000 & $\begin{array}{l}4,400,000 \\
1,200,000\end{array}$ \\
\hline $\begin{array}{l}310 \\
311\end{array}$ & Bloomer.......... & $2, \infty$ & 6 & 60 & $\begin{array}{r}1,200,000 \\
12,000\end{array}$ \\
\hline a312 & Ashland.... & 80 & 8 & 640 & 128,000 \\
\hline 313 & Hayward... & 40 & 16 & 640 & 128,000 \\
\hline 314 & .....do............. & 200 & 10 & 2,000 & 400,000 \\
\hline 315 & New Auburn..... & 1,000 & 8 & 8,000 & $1,600,000$ \\
\hline 316 & Cameron..... & 500 & 14 & 7,000 & $1,400,000$ \\
\hline 317 & Ladysmith.... & 40 & & 160 & 32,000 \\
\hline 318 & Heafford Junction. & 80 & 20 & 1,600 & 320,000 \\
\hline 319 & Minocqua............ & 40 & 6 & 240 & 48,000 \\
\hline 320 & .....do........ & & 16 & 1,280 & 256,000 \\
\hline & Lac du Fia & 15,000 & 4 & 60,000 & $12,000,000$ \\
\hline 322 & Powell... & 100 & & 800 & 160,000 \\
\hline 323 & Glidden....... & 300 & 14 & 4,200 & 840,000 \\
\hline 324 & Park Falls..... & 60 & 5 & 300 & 60,000 \\
\hline 325 & Kewaunee. & 800 & 6 & 4,800 & 960,000 \\
\hline 326 & 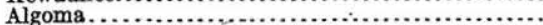 & 30 & 3 & 90 & 18,000 \\
\hline 327 & 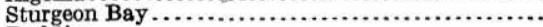 & 150 & 14 & 2,100 & 420,000 \\
\hline 328 & Peshtigo............ & 500 & & 1,000 & 200,000 \\
\hline 329 & Pembine............ & 80 & 6 & 480 & 96,000 \\
\hline 330 & Gagen & 200 & 9 & 1,800 & 360,000 \\
\hline 331 & 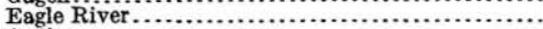 & 500 & 9 & 4,500 & 900,000 \\
\hline 332 & Antigo. & 50 & $1 \frac{1}{2}$ & 75 & \\
\hline 333 & Mountain........... & 60 & $3 \frac{1}{2}$ & 210 & 42,000 \\
\hline & & 121,220 & & 758,165 & $151,633,000$ \\
\hline
\end{tabular}

a Includes muck.

\section{MICHIGAN.}

\section{GENERAL FEATURES.}

The peat deposits of Michigan consist largely of "muskegs" and grass-sedge marshes in basins of glacial origin and are similar in most respects to those of Minnesota and Wisconsin. Except in Ontonagon and Baraga counties and in the southern part of Houghton County, peat is well distributed throughout the northern peninsula, but, as shown by Plate VIII, the most extensive deposits are in the eastern part of that peninsula. The largest swamps, some of which exceed 25 square miles in area, lie in a region about 90 miles long and 30 miles wide extending eastward from Munising toward Sault Ste. Marie. The peat in these swamps ranges from 2 to 20 feet in depth. Although there are many peat deposits in the southern peninsula of Michigan, few exceed 400 acres in area. The largest deposits occur in river valleys, and consequently contain a high percentage of ash. However, large quantities of good peat are found 


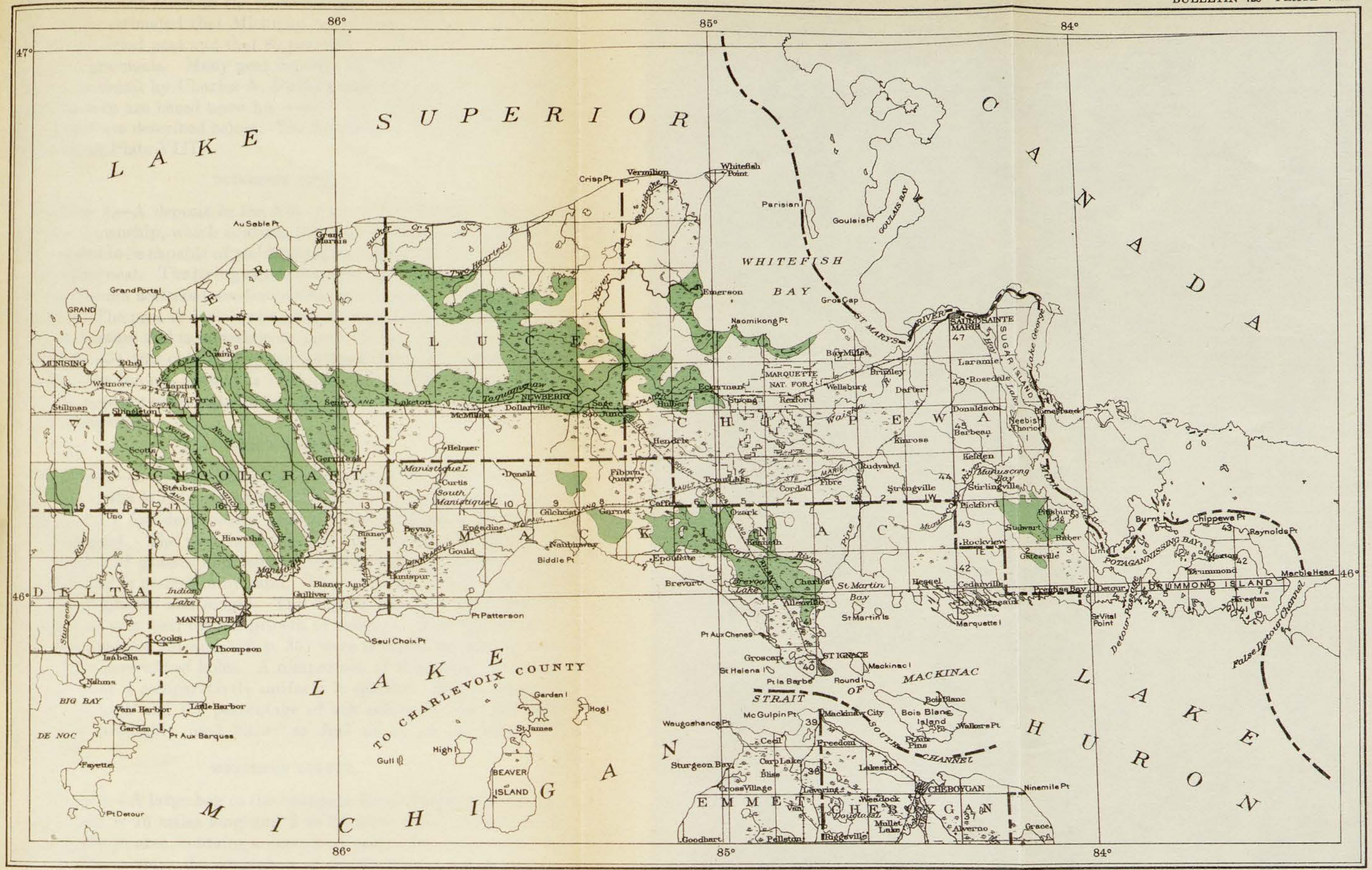

MAP SHOWING DISTRIBUTION OF PEAT IN THE EASTERN PART

OF THE NORTHERN PENINSULA OF MICHIGAN 

in nearly all the lakes in the northern part of the southern peninsula. It is estimated that Michigan could yield 1,000,000,000 short tons of air-dried peat and that 80 per cent of this quantity is in the northern peninsula. Many peat deposits in Michigan have been described in detail by Charles A. Davis, ${ }^{25}$ and many of the data presented herein are based upon his work. The deposits examined for this report are described below. The localities are indicated by their numbers on Plate VIII.

\section{DICKINSON COUNTY.}

Locality 1.-A deposit in the NE. $\frac{1}{4}$ sec. 7 and the NW. $\frac{1}{4}$ sec. 8 of Norway Township, which is about 130 acres in area and 5 feet deep, is estimated to be capable of yielding approximately 130,000 short tons of air-dried peat. The living vegetation consists chiefly of sedges and grasses, though scattered coniferous trees grow at the south end of the deposits. The peat is dark brown, well decomposed, and of relatively uniform texture and is underlain by sand. A typical sample (analysis 383, p. 35) was obtained by mixing peat taken from several test borings. As the ash content is 17.41 per cent and the nitrogen 1.89, the peat appears to be suitable for the production of only low-grade fuel and fertilizer.

\section{EATON COUNTY.}

Locality 2.-A deposit about $2 \frac{1}{2}$ miles east of Eaton Rapids, near the track of the Michigan Central Railroad, which is about 400 acres in area and 7 feet deep, would probably yield 560,000 short tons of air-dried peat. The peat is brown and fibrous, consists chiefly of the remains of grasses and sedges, contains many roots and pieces of wood, and is underlain by sand. The dominant living vegetation consists of grasses and sedges. The deposit has been well drained, and part of it is used for the growth of cabbage. Two typical samples (see analyses 351 and 352, p. 35) were obtained by mixing peat taken from several test holes. A comparison of these analyses shows that the peat is comparatively uniform in quality. As the nitrogen content is high and the percentage of ash relatively low, the peat might be profitably used either as fuel or as an ingredient of fertilizer.

\section{HOUGHTON COUNTY.}

Locality 3.-A large bog in the Sturgeon River Valley near Klingville, which is 16 miles long and 2 to $2 \frac{1}{2}$ miles wide and about 36 square miles in area, contains a deposit of peat 5 feet deep, which is estimated to contain the equivalent of $23,000,000$ short tons of air-

${ }^{25}$ Davis, C. A., Peat ; esays on its origin, uses, and distribution in Michigan : Michigan Geol. Survey Rept. for 1906, pp. 93-395, 1906. 
dried muck and peat. The deposit was sampled at points along a line extending from east to west through its center. Samples were also collected near the main drainage ditches along lines run at right angles to the other series of test borings. The upper 3 feet of peat is mossy and fibrous and contains many roots and pieces of wood; the lower layers are brown, well decomposed, soft, and plastic. The living vegetation consists chiefly of sphagnum moss, although tamarack, spruce, and swamp laurel are common. The deposit has been partly drained, and about 300 acres have been cleared. A representative sample (analysis 372, p. 35 ) was obtained by mixing muck and peat taken at intervals of 2 feet in depth in eight test holes. Analyses 373 and 375 (p. 35) or both the raw and macerated muck and peat show that they contain too much ash for fuel. The nitrogen content is a little less than.2 per cent and is relatively uniform throughout the deposit.

Locality 4.-In a small bog in the NE. $\frac{1}{4}$ sec. 22 and the NW. $\frac{1}{4}$ sec. 23, Calumet Township, the living vegetation consists chiefly of tamarack and small poplar trees, although it includes some tufts of sphagnum moss. The peat is brown and fibrous and contains much woody material. Four test borings were made, and a typical sample (analysis 378, p. 35) was obtained by mixing peat taken from each. The general appearance of this bog is misleading. Its topographic position and flora justify the assumption that it contains a large quantity of peat, but test borings show only a shallow accumulation of fibrous plant remains. There are many peat bogs of this type in the vicinity of Calumet and Osceola.

Locality 5.-A small bog near the tracks of the Duluth, South Shore \& Atlantic Railway, west of the Hecla mine, contains a deposit which is 15 acres in area and 12 feet in average thickness. The upper layer of the peat has shrunk, producing an open fissure and exposing a cross section of the deposit. The peat consists chiefly of remains of grass, is brown, coarse, and fibrous, and is underlain by sand. A sample (analysis 379, p. 35) was obtained from the exposed section. As its nitrogen content is unusually high (2.53 per cent), the peat might be used as fertilizer, but though its ash content is low fuel could not be economically made from it, because the bog has been drained to the bottom and the peat is too dry and fibrous to be shaped into blocks.

\section{IRON COUNTY.}

Locality 6.-A small bog near the center of sec. 22, T. 43 N., R. $32 \mathrm{~W}$., contains a deposit about 6 acres in area and 4 feet deep. The living vegetation consists chiefly of sedges and sphagnum. The peat, which is composed of the remains of plants like those growing 
in.the bog, is brown, fibrous, and woody. Analysis 380 (p. 35) shows the quality of the peat in this bog. Similar bogs occupy small depressions, known as kettle holes, in this vicinity.

Locality 7.-A deposit in the NW. $\frac{1}{4}$ sec. 21 and the SW. $\frac{1}{4}$ sec. 16 , Crystal Falls Township, is about $90^{\prime}$ acres in area and 3 feet deep: The living vegetation consists chiefly of sedges and reeds, though some trees grow at the north end. The peat is dark brown and well decomposed but not plastic, and consists chiefly of the remains of sedges and reeds. Four test borings were made at intervals of 100 yards at points along a line extending from north to south through the center. An average sample (analysis 381, p. 35) was obtained by mixing peat taken at several depths from each of the test holes.

Locality 8.-A bog east of Ice Lake in the NE. $\frac{1}{4}$ sec. 30 , the western part of sec. 29, the SE. $\frac{1}{4}$ sec. 19, and the SW. $\frac{1}{4}$ sec. 20, Bates Township (T. 43 N., R. 34 W.) is about 120 acres in area and 3 feet in average depth. The deposit is composed chiefly of the dark-brown and fibrous remains of mosses, reeds, and sedges. The living vegetation consists principally of sphagnum and heath shrubs. A typical sample (analysis 382, p. 35) was obtained by mixing material taken from numerous test holes. The high content of inorganic mineral matter (41.55 per cent) shows that the deposit contains muck which is unfit for fuel.

\section{RATAMAZOO COUNTY.}

Locality 9.-A deposit near the Grand Rapids \& Indiana Railway north of Vicksburg, which is about 400 acres in area and 5 feet in average depth, is capable of yielding 400,000 short tons of air-dried peat and muck. The surface layer consists of black muck, and the underlying peat ranges from brown to black, is rather fibrous, and is underlain by sand. It occupies a basin-shaped depression that would be difficult to drain. The living vegetation consists chiefly of sedges and grasses. A representative sample (analysis 347, p. 35) was obtained by mixing peat and muck taken from four test borings made at points along a line crossing the middle of the deposit. The heating value of the moisture-free peat is 8,203 British thermal units, the ash content 12.71 per cent, and the nitrogen 3.58 per cent. The peat, therefore, seems well adapted for fuel or fertilizer.

Locality 10.-A deposit on the Kleinstueck estate, in the southern part of the city of Kalamazoo, is about 30 acres in area and 6 feet in average thickness. The peat is brown and rather fibrous and is underlain by sand. Several test borings were made near the center of the deposit, and a representative sample (see analysis 350, p. 35) which was obtained by mixing peat from each of the test holes, shows that the peat is of excellent quality for fuel or fertilizer. Analyses 348 and 
349 (p. 35) show the quality of dried and pressed peat from this deposit. A comparison of these analyses shows that the deposit con. tains peat of relatively uniform composition:

IAPEER OOUNTY.

Locality 11.-A deposit south of the Grand Trunk Railway tracks, a short distance east of Imlay City, is about 200 acres in area and 8 feet in average depth. The living vegetation consists chiefly of grasses and sedges and near the south end of scattered tamarack trees. The peat is dark-brown, well-decomposed, and plastic, contains some woody matter, and is underlain by sand. Three test holes were made at intervals of 100 yards across the south end. A representative specimen (analysis 357, p. 35) was obtained by mixing peat taken at intervals of 2 feet in depth, from each of these borings. The calorific value of the moisture-free peat is 9,186 British thermal units and the nitrogen content 2.24 per cent. The peat should therefore make good fuel and fertilizer.

\section{LUCE COUNTY.}

Locality 12.-A bog near the Duluth, South Shore \& Atlantic Railway west of Newberry, about 150 acres in area, contains a deposit of peat 4 feet in average depth. The peat consists chiefly of the remains of sedges and moss, contains several thin layers of woody material, and is dark brown and well decomposed. The living vegetation consists of spruce, tamarack, sedges, sphagnum, and numerous varieties of small shrubs. A typical sample (analysis 369, p. 35) was obtained by mixing peat taken at different depths from four test holes made across the center of the bog.

Locality 13.-A large swamp north of Newberry, which is several hundred acres in area, contains peat only 2 feet in average depth. The living vegetation consists of spruce, tamarack, and a dense growth of shrubs. The peat, which was produced chiefly by the decay of sedges and grasses, is dark brown to black, is well decomposed, and is underlain by sand. Analysis 370 (p. 35) shows that the peat is of rather poor quality.

\section{MARQUETTE COUNTY.}

Locality 14.-A bog in the northern part of the city of Marquette, near the shore of Lake Superior, about 300 acres in area, contains a deposit of peat 2 feet in average thickness. The living vegetation consists chiefly of grass, moss, and scattered coniferous and poplar trees. The peat is black and ranges in texture from fibrous to plastic. Analysis 371 (p. 35) shows that it is fair in quality, but the deposit is probably too shallow to be of economic value. 
MECOSTA COUNTY.

Locality 15.-A large swamp north and east of the village of Mecosta contains a deposit of peat about 3,500 acres in area and from 4 to 16 feet in thickness. This deposit is capable of yielding approximately 4,900,000 short tons of air-dried peat and muck. The peat and muck are dark brown, fibrous, and woody and are underlain by sand and marl. The living vegetation consists chiefly of poplar, spruce, and tamarack trees. As there is a dense undergrowth of small shrubs, the swamp is difficult to penetrate. The surface is thickly covered with old logs, stumps, slashings, chips, and other débris of logging operations. A series of test borings was made at intervals of 100 yards along an east-west line across the center of the swamp. Representative samples (analyses 365, 366, 367, 368, p. 35) were obtained from these test borings by mixing material taken at different depths. A comparison of the analyses shows that the deposit contains too much ash for good fuel. The nitrogen content, which ranges from 1.42 per cent to 1.97 per cent, is also low. The high sulphur content (3.48 per cent in analysis 366$)$ is noteworthy.

\section{MUSKEGON COUNTY.}

Locality 16.-A muck marsh along Muskegon River, in the northeastern part of Muskegon Township, contains a deposit of peat 3,000 acres in area and 5 feet in average thickness. The depth, however, is variable, and small pockets were encountered in sampling the deposit in which the organic matter was much more than 5 feet deep. The living vegetation consists of a heavy growth of marsh grass and bulrushes. At the north end of the marsh beech and willow trees are numerous. The muck is brown and fibrous and contains a large percentage of inorganic material, owing to the large quantities of sand deposited by Muskegon River during floods. The recurrent floods which have covered this area are recorded in the deposit by alternate layers of sand and organic matter. Numerous test borings were made along both the north and south sides of the marsh and a representative sample (analysis 364, p. 35) was obtained by mixing material taken from the test holes. According to the analysis, the sample contains 58.32 per cent of ash and 1.57 per cent of nitrogen, and is therefore to be classed as muck rather than peat. Louis P. Haight, of Muskegon, is reclaiming this marsh for agricultural use.

OAKLAND COUNTY.

Locality 17.-A deposit near the center of sec. 32 , in the southern part of Pontiac, occupies an area of about 40 acres and is $2 \frac{1}{2}$ feet in average depth. The living vegetation consists of sedges and 
reeds. The peat is dark brown, well decomposed, and plastic, consists principally of the remains of sedges, and is underlain by marl. Several borings were made throughout the area, and a representative sample (analysis 356, p. 35 ) was obtained by mixing the peat from the test holes. The peat contains an unusually large proportion of nitrogen (3.22 per cent) but is not thick enough to be workable com. mercially.

ST. CLAIR DOUNTY.

Locality 18.-A large marsh on the Grand Trunk Railway 3 miles west of Capac contains a deposit about 3,000 acres in area and 7 feet in average depth. The peat is brown and fibrous and is underlain by sand. The living vegetation consists chiefly of grasses, sedges, reeds, and the blueberry. Representative specimens (analyses 358, 359,360, p. 35 ) were obtained by mixing peat taken at numerous places along the drainage ditch that extends longitudinally across the marsh. The peat is low in ash and high in caloriftc value, but it is too fibrous for the manufacture of machine-peat fuel.

SHIAWASSEE COUNTY.

Locality 19.-A bog about 2 miles west of Bancroft, which is 300 acres in area, contains a deposit 5 feet in average thickness. The living vegetation consists chiefly of sedges, grasses, reeds, bulrushes, and tamarack trees. The peat is dark brown to black, well decomposed, and plastic, and is underlain by impure marl and sand. Numerous test borings were made through the center, and composite samples (analyses 361, 362, 363, p. 35) were obtained by mixing peat from each of the test holes. The peat appears to be of good quality for fuel or for fertilizer.

WASHTENAW COUNTY.

Locality 20.-A bog in the southern part of Chelsea, about 100 acres in area, contains a deposit of peat 3 feet in average depth. The living vegetation consists chiefly of grasses, mosses, and the blueberry. The peat is brown, fibrous, and flaky and is underlain by impure marl and sand. Test borings were made at intervals of 50 yards across the center of the bog, and typical samples (analyses 353, 354,355, p. 35 ) were obtained by mixing peat taken from each hole. A comparison of the analyses shows the peat is uniform in composition and appears to be of the proper quality for fuel or fertilizer, but it is probably too shallow for commercial use.

IOWA.

The principal peat deposits in Iowa occur in the Altamont morainal belt of the Wisconsin glacial drift and are largely confined 
to the north-central part of the State. Cerro Gordo, Clay, Dickinson, Emmet, Franklin, Hamilton, Hancock, Kossuth, Palo Alto,

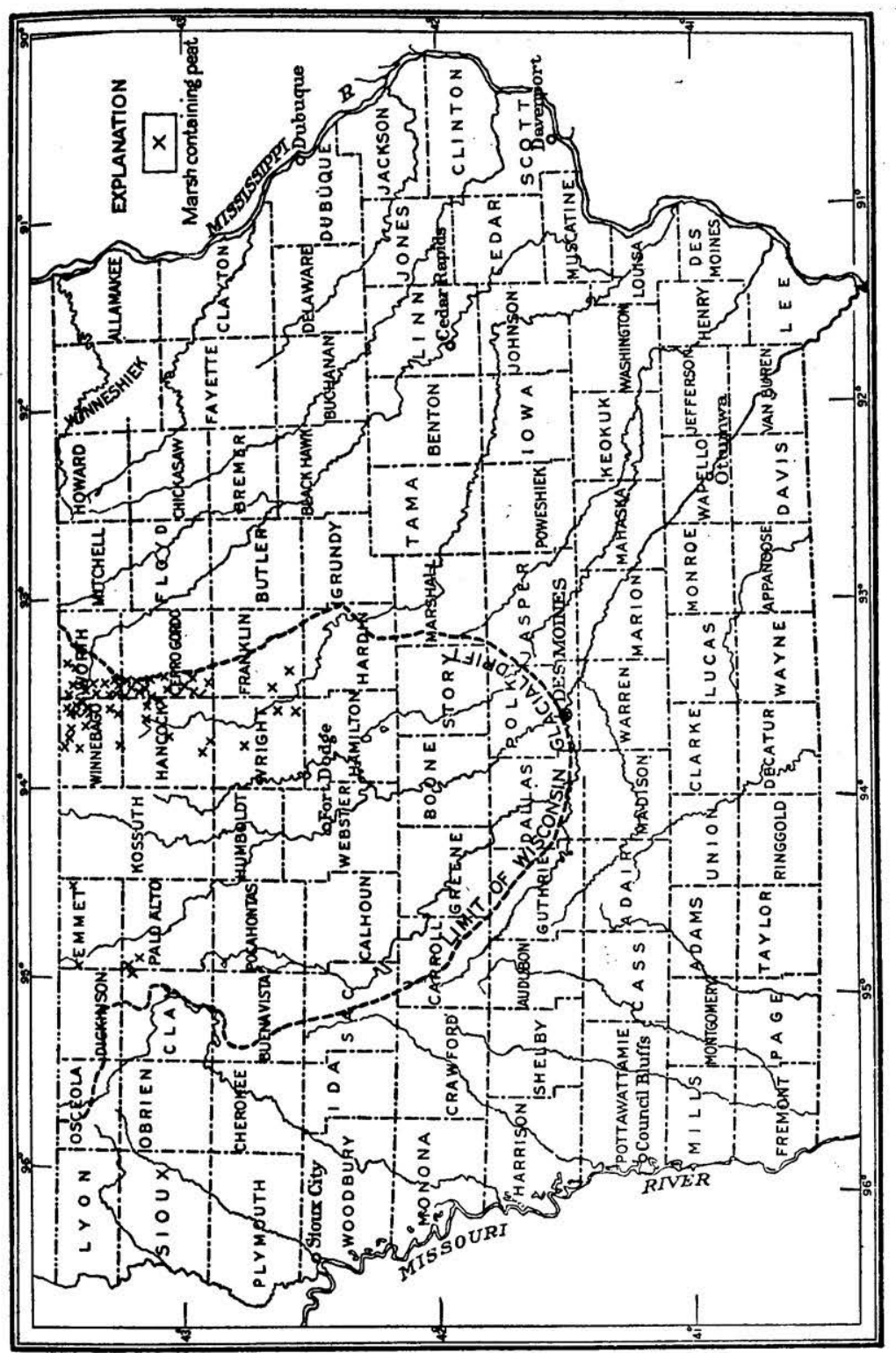

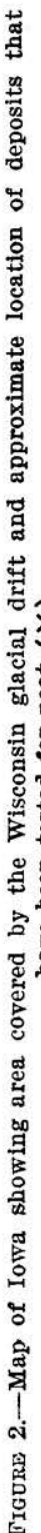

Webster, Winnebago, Worth, and Wright are the counties in which peat deposits are most extensive. (See fig. 2.) Most of the peat was formed in open grass-sedge marshes and in shallow lakes and ponds. Sphagnum and other mosses were not large contributors to peat in Iowa. The deposits range from 1 to 150 acres in area and 
from 1 to 20 feet in depth. Savage ${ }^{26}$ estimates that Iowa peat bog would yield $22,000,000$ short tons of air-dried peat. The depos that exceed 40 acres in area are described in reports ${ }^{27}$ issued by th Iowa Geological Survey. (See p. 29 for analyses.)

IIINOIS.

GENERAI FEATURES.

The peat deposits of Illinois originated in hollows in the glaci drift and in lowlands near lakes and rivers. Grassy meadows an grass-sedge and cat-tail marshes are the most common type There are no wooded swamps in Illinois comparable with those Minnesota, Wisconsin, and Michigan. Most of the deposits an small, but several that exceed 1,000 acres in area are found in Whit side County and in a few other parts of the State. The thicknes of the peat ranges from 2 inches to 20 feet, but the average dept of most of the deposits is less than 5 feet. Du Page, Kane, Kar kakee, Lake, Lee, McHenry, Mason, Rock Island, Tazewell, Whit side, and Winnebago counties contain practically all the workabl peat in Illinois. Although the largest area of peat, known as $\mathrm{Ca}$ tail Slough, is in Whiteside County, in Cattail Valley, southea: of Fulton, more peat occurs in Lake than in any other count, Plate IX shows the distribution of peat and muck in Lake Count and figure 3 (p. 111) shows Cattail Valley in Whiteside Count: One of the largest deposits in the State is situated near Manita Mason County. According to the most reliable data available Ill: nois peat bogs would yield about $10,000,000$ short tons of air-drie peat, the distribution of which in the counties that contain th largest quantities is shown in the following table: ${ }^{28}$

Distribution of peat and muck in six counties of Illinois.

\begin{tabular}{|c|c|c|}
\hline \multirow[b]{2}{*}{ County. } & \multicolumn{2}{|c|}{ Ares. } \\
\hline & $\begin{array}{l}\text { Square } \\
\text { miles. }\end{array}$ & Acres. \\
\hline 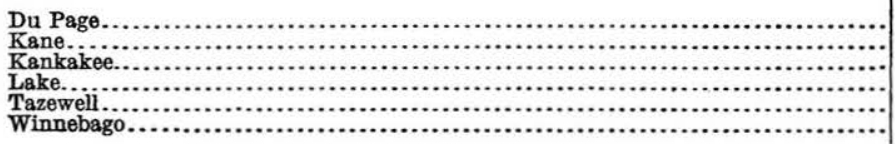 & $\begin{array}{r}6.54 \\
14.53 \\
2.73 \\
38.10 \\
2.10 \\
2.23\end{array}$ & $\begin{array}{r}4,18 \\
9,29 \\
1,74 \\
24,34 \\
1,34 \\
1,42\end{array}$ \\
\hline & 66.23 & $42,3 \pi$ \\
\hline
\end{tabular}

The following localities were tested for peat in the course of field work for this report:

${ }^{20}$ Savage, T. E., A preliminary report on the peat resources of Iowa : Iowa Geol. Surves Bull. 2, p. 8, 1905 .

${ }^{2}$ Beyer, S. W., Peat deposits in Iowa : Iowa Geol. Survey Ann. Rept., vol. 19, pp. 689. 730, 1908. Savage, T. E., op. cit.

${ }^{28}$ Mopkins, C. G., Mosier, J. G., Van Alstine, E., and Garrett, F. W., Illinois Univ. Agt. Exper. Sta. Soil Repts. Nos. 9, 12, 13, 14, 16, 17, etc., 1915-1917. 
R.9 E. R.10E.W I S C O N S I N R.IIE.

R.12 E.

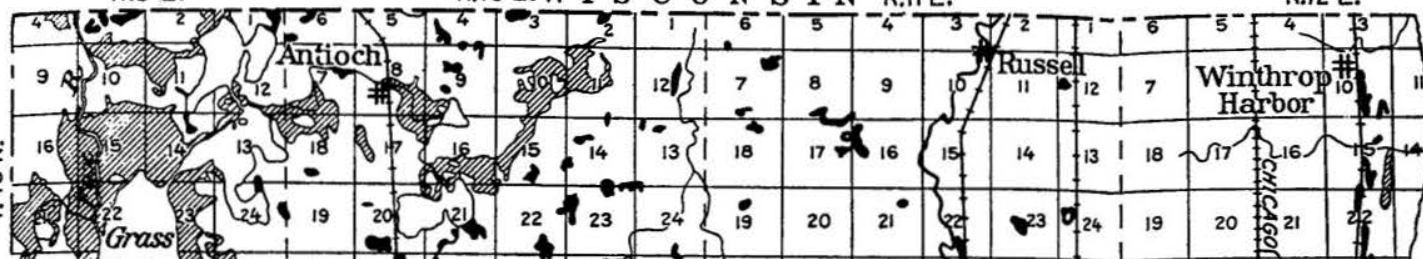

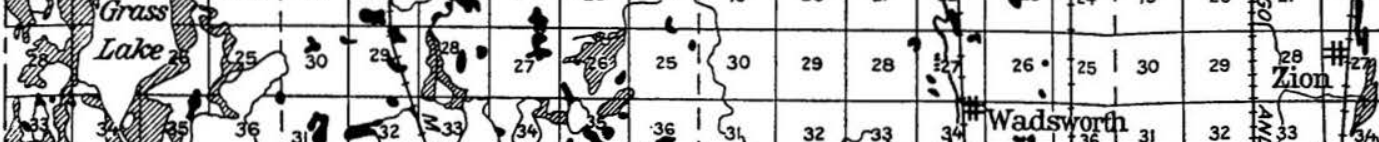

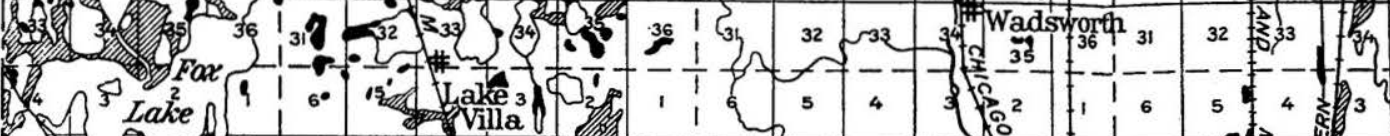

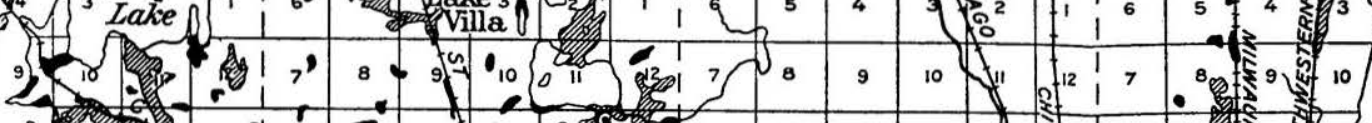

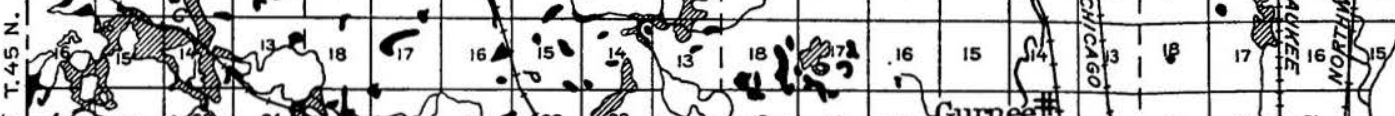

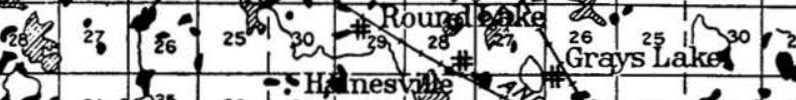

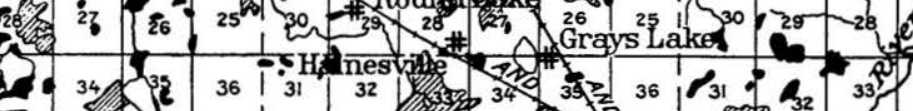
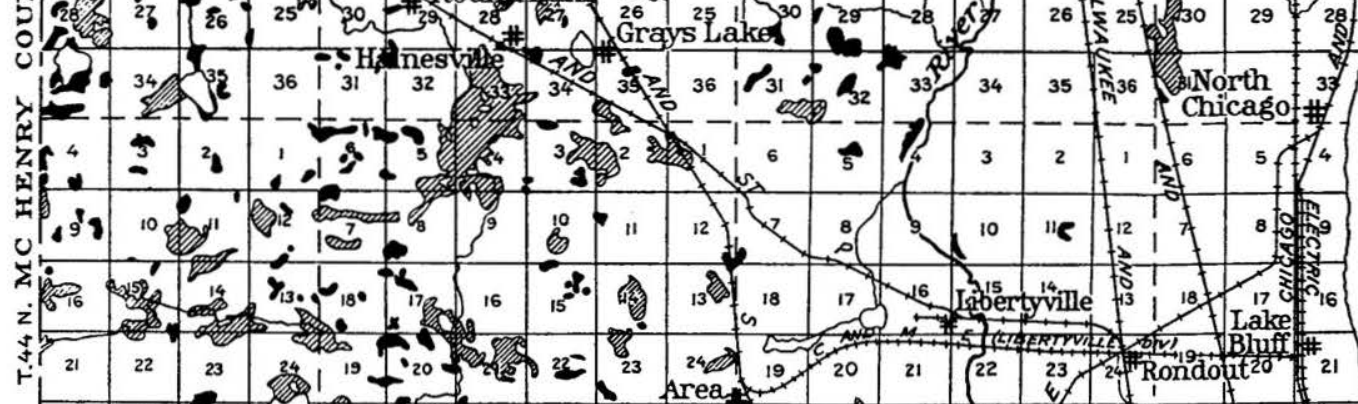

\section{16. Itibertyville}

$\rightarrow$ 标

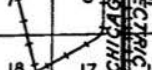

18 18 Lake 16

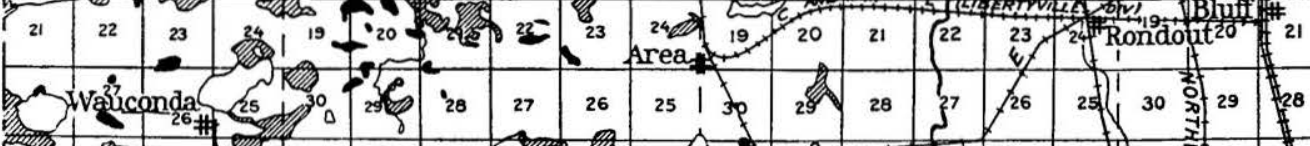

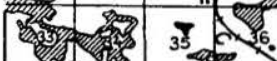

35 .

33

36

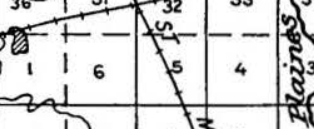

है।

3536 गी 31

2 - 11 th

1217 Fort

今

A

iy

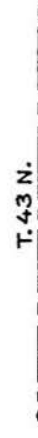

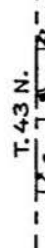
pon

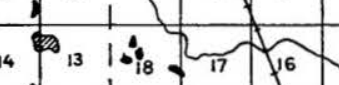
. 28

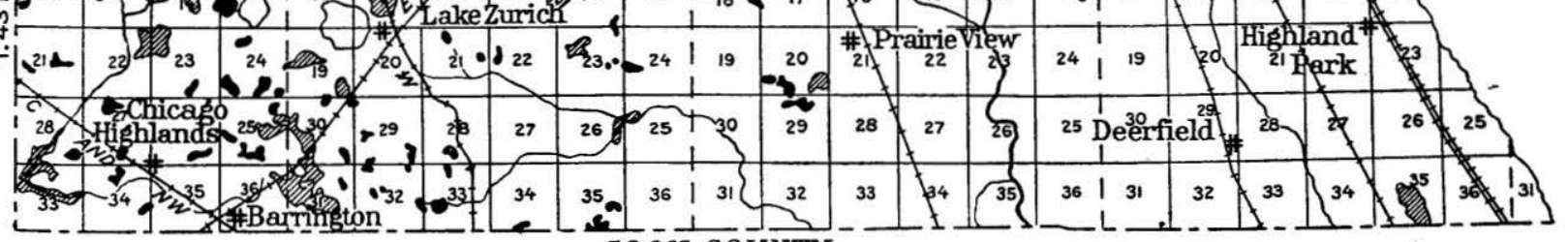
coor couvry

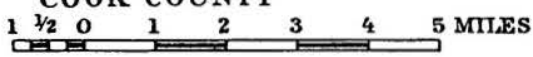

MAP SHOWING DISTRIBUTION OF PEAT AND MUCK IN LAKE COUNTY, ILL. RULED AND BLACK AREAS CONTAIN PEAT OR MUCK. 

RANE OOUNTY.

Locality 1.-A marsh about 2 miles south of Aurora which occupies part of secs. 26 and 27 , T. 38 N., R. $8 \mathrm{E}$., is about 120 acres in area and contains a deposit of peat 3 feet in average depth. Much of the peat has been destroyed and the remainder is very irregular in thickness and is gritty and impure because of the sand and clay that it contains. The living vegetation is sedges, grasses, and stunted willows, and the peat consists chiefly of the remains of these plants. Several test holes were made, but as no peat of economic value was found samples were not taken for analysis. The peat in this locality is owned by H. H. Evans, of Aurora, Ill.

\section{KANKAKEE OOUNTY.}

Locality 2.-A deposit of peat in the SW. $\frac{1}{4}$ sec. 35, T. 32 N., R. $11 \mathrm{~W}$., is about 80 acres in area and 4 feet in average depth. The peat occurs in pockets separated by areas of dry land, a condition typical of the surrounding region. It is composed chiefly of the remains of sedges and grasses and, except the surface layer, is black, plastic, and well decomposed. Grasses and sedges are the dominant living vegetation. Test holes were sunk at intervals of 100 yards along an east-west line crossing the center of the deposit. Samples were taken at depths of 2 feet and mixed in order to obtain the representative specimen whose composition is shown in analysis 317 (p. 27). The peat at the bottom of the deposit is black and is underlain by sand and clay. The high nitrogen content (3.03 per cent) is noteworthy, and the peat appears to be valuable as a nitrogenous ingredient of commercial fertilizers. The ash content is so high that the deposit can not be recommended as a source of fuel.

\section{IAKE COUNTY.}

Locality 3.-A deposit northwest of Duck Lake, in secs. 14 and 15, T. $45 \mathrm{~N}$., R. 9 E., is about 90 acres in area and 6 feet in average thickness. It would yield approximately 108,000 short tons of airdried peat. The Chicago, Milwaukee \& St. Paul Railway is parallel to the northern edge of the deposit. The peat is black, plastic, and well decomposed. It is comparatively free from sand and silt and is underlain by marl. Sedges and grasses contributed the vegetable débris from which the peat was formed and also constitute the predominant living vegetation. The following results were obtained from test borings:

Thickness of peat in test borings northwest of Duck Lake, Lake County, Ill. Feet.

Hole A, 50 yards from west edge: Black plastic well-decom-posed peat

Hole B, 100 yards east of hole A: Black plastic well-decomposed peat 
Samples were taken at intervals of 2 feet in depth and mixed to obtain a representative specimen (analysis 300, p. 27 ). The nitro. gen content ( 3.84 per cent) is unusually high, and the ash content (15.14 per cent) relatively low. This deposit is well situated with respect to transportation facilities and market, possesses good quali. ties for fertilizer, and therefore offers an excellent opportunity for commercial development.

Locality 4.-A deposit south of Fox Lake, in the NW. $\frac{1}{4}$ sec. 11, T. 45 N., R. 9 E., is about 80 acres in area, 10 feet in average thick. ness, and would yield 160,000 short tons of air-dried muck. The muck is black, plastic, and well decomposed and consists chiefly of the remains of sedges and grasses. These plants also constitute the dominant living vegetation. The following test borings were made:

Thickness of muck in test borings south of Fox Lake, Lake County, Ill.

Hole A, 20 yards east of west edge: Black plastic welldecomposed muck

Feet.

Hole B, 100 yards east of hole A : Black plastic well-decomposed muck

A composite specimen (analysis 301, p. 27) was obtained by mix. ing samples of muck taken at intervals of 2 feet in depth in each hole. The ash content ( 47.40 per cent) is high, and the muck is therefore valueless for fuel, but the nitrogen content, which is 2.18 per cent, indicates that the material might be valuable for use as 8 nitrogenous ingredient of commercial fertilizers.

Locality 5.-A deposit near the railroad station at Antioch, be. tween Silver Lake and the tracks of the Minneapolis, St. Paul \& Sault Ste. Marie Railway, which occupies parts of secs. 8, 9, 16, and 17 , T. 46 N., R. 10 E., is several hundred acres in area, but only about 60 acres contain peat more than 3 feet thick. The 60 -acre tract contains peat 5 feet in average thickness and would yield approximately 60,000 short tons of air-dried peat consisting of black nonfibrous plastic remains of sedges, grasses, swamp ferns, and reeds. The liv. ing vegetation is composed of sedges, grasses, reeds, cat-tails, and swamp ferns. The following test borings were made:

Thickness of peat in test borings near Antioch, Lake County, Ill.

Hole A, 60 yards from east edge, near railroad station: Black plastic well-decomposed peat, underlain by sand Feet.

Hole B, 150 yards west of hole A: Black plastic well-decomposed peat, underlain by sand

A typical sample (analysis 302, p. 27) was obtained by mixing peat taken at intervals of 2 feet in depth from both test holes. The ash content is 21.74 per cent, the calorific value 7,756 British thermal units, and the nitrogen content 2.85 per cent. The deposit can not be recommended as a source of fuel, but if it were properly 
drained the peat might be profitably used for the cultivation of acidtolerant crops or for an ingredient of commercial fertilizer.

Locality 6.-A marsh about $2 \frac{1}{2}$ miles west of Antioch, in secs. 12 and 13, T. 46 N., R. 9 E., contains a deposit approximately 100 acres in area and 6 feet in average thickness, which would yield approximately 120,000 short tons of air-dried peat. The living vegetation consists chiefly of sedges, marsh grass, reeds, and rushes, though pond lilies are numerous along some of the channels. The peat is brown, fibrous, coarse, and spongy, and is composed principally of coarse sedge and grass roots and reeds. Samples were taken along the banks of a large drainage ditch that crosses the marsh, and a representative specimen (see analysis 303, p. 27) was obtained by mixing the material from the different test holes. The ash content is very low (9.12 per cent), but the peat is too fibrous and spongy for fuel. The nitrogen content (2.52 per cent) suggests that the peat might be valuable for agricultural use. Large quantities of moss suitable for stable litter or packing material could readily be obtained at this locality.

Locality 7.-A large marsh north of Grass Lake, about $3 \frac{1}{2}$ miles west of Antioch, in secs. 14 and 15, T. 46 N., R. 9 E., contains a deposit 400 acres in area and $5 \frac{1}{2}$ feet in average thickness, which would yield approximately 440,000 short tons of air-dried muck. On June 20,1918, this marsh was flooded by water from Grass Lake and Fox River and was therefore difficult of access. The surface is overgrown by giant sedges and associated reeds and rushes. The muck is black, soft, and plastic, and is underlain by marl. The following test borings were made at points along an east-west line through the center :

\section{Thickness of muok in test borings in marsh north of Grass Lake, Lake County, $\mathrm{nl}$.}

Hole A, 100 yards west of east edge; black well-decomposed plastic muck, underlain by marl

reet.

Hole B, 100 yards west of hole A; black well-decomposed soft, plastic muck, underlain by marl

Hole C, 200 yards west of hole B; black well-decomposed soft, plastic muck, underlain by marl

Hole D, 100 yards west of hole C; black well-decomposed soft, plastic muck, underlain by marl

A composite sample (analysis 304, p. 27) was obtained by mixing peat taken at intervals of 2 feet in depth from each of the four test holes. The ash content (41.97 per cent) indicates that the material in this deposit is unfit for fuel, but the nitrogen content (2.45 per cent) is relatively high and suggests that the deposit may be valuable for agricultural use. The sedge growth is suitable for the production of packing material. 
Locality 8.-A deposit about 1 mile west of Antioch, in secs. 7 and 18, T. 46 N., R. 10 E., which is 120 acres in area and 8 feet in average thickness, would yield about 192,000 short tons of air-dried peat. The peat is black and consists chiefly of the remains of sedges, grasses, swamp ferns, and moss. The living vegetation is composed of sedges and swamp ferns. The following test borings were made at intervals of 100 yards along a line extending from the northeast to the southwest corner of the deposit, as well as at a point 100 yards northwest of the center:

Thickness of peat in test borings in deposit 1 mile west of Antioch, Ill.

Hole A, 100 yards southwest of northeast corner; black, compact, well-decomposed peat, underlain by marl_-_-_-_-_-..- 5

Hole B, 100 yards southwest of hole A; black well-decomposed peat, underlain by marl Feet.

Hole C, 100 yards southwest of hole B; black well-decomposed peat, underlain by marl.

Hole D, 100 yards southwest of hole $\mathrm{C}$; black well-decomposed peat, underlain by marl

Hole E, 100 yards northwest of center of deposit; black welldecomposed peat, underlain by marl 5

A composite sample (analysis 305, p. 27) was obtained by mixing peat obtained at intervals of 2 feet in depth from each of the test holes. The ash content is 20.61 per cent and the nitrogen 3.18 per cent. The deposit can not be recommended as a source of fuel, but on account of the large percentage of nitrogen it should be valuable for agricultural use.

Locality 9.-A small deposit on the Lynnhurst farm, about $2 \frac{1}{2}$ miles east of Lake Villa, in the center of sec. 35 , T. 45 N., R. 10 E., is 30 acres in area, 12 feet in average thickness, and would yield approximately 72,000 short tons of air-dried peat. The living vegetation consists chiefly of sedges, swamp ferns, and moss, and the peat is brown, fibrous, and spongy. A test boring made near the center of the deposit gave the following results:

Log of test boring near center of deposit on Lymnhurst farm, Lake Villa, Ill.

$\begin{array}{lr}\text { Brown fibrous spongy mat, composed of roots of living plants_- } & 1 \\ \text { Brown fibrous peat, little decomposed.- } & 2 \\ \text { Brown, partly disintegrated peat, firm but fibrous_- } & 4 \\ \text { Brown soft, plastic peat, underlain by sand.-- } & 5\end{array}$

Analysis 306 (p. 27) shows that the peat contains only 10.6 per cent of ash and that the nitrogen content is 2.88 per cent. The deposit could therefore be used for the production of fuel or fertilizer, especially the lower layers.

Locality 10.-A deposit of the meadow type, about 1 mile south of Hainesville, on the Chicago, Milwaukee \& St. Paul Railway, in secs. 33 and 34, T. 45 N., R. 10 E., is about 400 acres in area. The vege- 
tation consists chiefly of grasses, although bulrushes grow in some of the lower and wetter spots. No peat of economic value was found and hence no samples were taken for analysis. This area could perhaps be used for the cultivation of crops.

LEE COUNTY.

Locality 11.-Several small meadows, about 4 miles southeast of Amboy, each of which is between 20 and 30 acres in area, contain muck, but no peat of economic value was found in this locality.

Locality 12.-Several large meadows or marshes along the Green River valley contain a little peat, but most of the material consists of shallow muck. No samples were taken for analysis.

\section{MASON AND TAZEWELI COUNTIES.}

Localities 13 and 14.-A large deposit occupies parts of secs. 15, 16,21 , and 22, T. 23 N., R. 6 W., near Manito. The material in the western and eastern parts of this deposit is different in thickness and quality.

The eastern part of the deposit is 120 acres in area and 5 feet in average thickness and would yield approximately 120,000 short tons of airdried peat. The living vegetation is composed of grasses and the milkweed, and the peat, which is largely black and plastic, consists of the remains of these plants. The lower layers are so thoroughly decomposed that the plant remains are difficult to identify. Five test borings made along north-south and east-west lines through the center of the deposit gave the following results:

Thickness of peat in test borings in central part of deposit near Manito, Ill.

Hole A, 100 yards east of west edge: Brown, fibrous peat in upper layers to black plastic peat at bottom, underlain by sand

Feet.

Hole B, 100 yards east of hole A : Same kind of peat as that in hole $\mathrm{A}$, underlain by sand

Hole C, 100 yards east of hole B : Material of same character as that obtained in hole $\mathbf{B}$.

Hole D, 100 yards south of north edge: Same kind of peat as that in hole $\mathrm{C}$, underlain by sand.

Hole E, 100 yards south of hole D: Material of same character as that in hole $\mathrm{D}$.

According to analysis 313 (p. 27), the ash content of the peat is 26.57 per cent and the nitrogen 2.24 per cent. Although this part of the deposit can not be recommended as a source of fuel, the peat is especially suitable for direct use as a fertilizer or for a nitrogenous ingredient of commercial fertilizers.

The western part of this deposit is about 240 acres in area and 3 feet in average depth and contains the equivalent of about 144,000 short tons of air-dried muck. The living vegetation is similar to 
that in the eastern part, and the muck is black and plastic and is underlain by clay and sand. Three test borings gave the following results:

Thickness of peat in test borings in western part of deposit near Manito, IIl.

Hole A, 400 yards east of plant of Weidmer Chemical Co.: Feet. Black plastic well-decomposed muck, underlain by sand----

Hole B, 100 yards south of hole A : Black plastic well-decomposed muck, underlain by clay

Hole C, 100 yards south of hole B : Black plastic well-decomposed muck, underlain by sand

According to analyses 314,315 , and 316 (p. 27), the muck seems to be well adapted for use as a nitrogenous fertilizer.

\section{WHITESIDE COUNTY.}

Locality 15.-Cattail Slough, near Sollers, in Cattail Valley, in secs. $6,7,8,17,18,19,20,28,29,32$, and 33 , T. 21 N., R. 4 E., contains a deposit several square miles in area, but the part here considered embraces only 1,280 acres near the south end of Cattail Valley, in secs. 28, 29, 32, and 33. (See fig. 3.) The average thick. ness of the peat in this part of the deposit, which would yield approximately $3,840,000$ short tons of air-dried material, is about 15 feet. The living vegetation consists of marsh grasses, sedges, bulrushes, reeds, and cat-tails. The upper layers of peat are brown and fibrous, but the underlying strata are well decomposed and plastic.

Test-borings made along two intersecting lines extending southwest and southeast through the center of the area gave the following re. sults:

Thickness of peat in test borings in Cattail Slough, Whiteside County, Ill.

Hole $\mathrm{A}$, about 150 yards northeast of road along western edge of deposit: Brown fibrous to black plastic decomposed peat, underlain by sand.

Hole B, 450 yards northeast of hole A : Brown flbrous to black decomposed peat, underlain by sand

Hole C, 450 yards northeast of hole B : Brown flbrous peat, underlain by marl containing snail shells.

Hole D, 450 yards northeast of hole C: Brown flbrous to black decomposed peat, underlain by marl containing snail shells_-

Hole E, 450 yards southeast of hole $C$, along a line at right angles to holes A, B, C, and D: Brown fibrous peat, rather woody near bottom, underlain by sand.

Hole F, 450 yards northwest of hole C: Brown fibrous to black, partly disintegrated peat, underlain by marl and sand. 
Hole G, 450 yards northwest of hole F : Brown fibrous to black, partly disintegrated peat, underlain by sand___________ 15

Hole $\mathbf{H}, \mathbf{4 5 0}$ yards northwest of hole $\mathrm{G}$ : Brown fibrous to black, partly disintegrated peat, underlain by sand_-_________- 16

Hole I, 450 yards northwest of hole $H$ : Brown fibrous to black, well-decomposed peat, underlain by sand and marl.

Hole J, 450 yards northwest of hole I : Brown fibrous to black, well-decomposed peat, underlain by marl and sand.

Feet.

The quality of the peat from holes A, B, C, and D is shown by analysis 307 ; from holes $\mathrm{E}, \mathrm{F}, \mathrm{G}$, and $\mathrm{H}$ by analysis 308 , and from holes $I$ and $J$ by analysis 309 . (See p. 27.) A comparison of these analyses shows that the peat contains relatively little ash, is high in calorific value, and therefore might be advantageously used for fuel. . The high average nitrogen content of the peat (3.06 per cent) also indicates that it is" suitable for fertilizer. This is one of the most valuable peat deposits in Illinois. Much of the area has already been drained and is used for farming. The Clinton branch of the Chicago, Burlington \& Quincy Railroad crosses the deposit, and when trains are passing the surface quakes violently.

Locality 16.-A deposit in the upper Cattail Valley, including parts of secs. $6,7,8,17$, and 18 , T. 21 N., R. 4 E., comprises about 1,300 acres of peat 12 feet in average depth and would yield approximately $3,120,000$ short tons of , air-dried peat. The living vegetation consists chiefly of cat-tails, bulrushes, sedges, grasses, and reeds. The peat is brown and fibrous near the surface, but the underlying strata are well

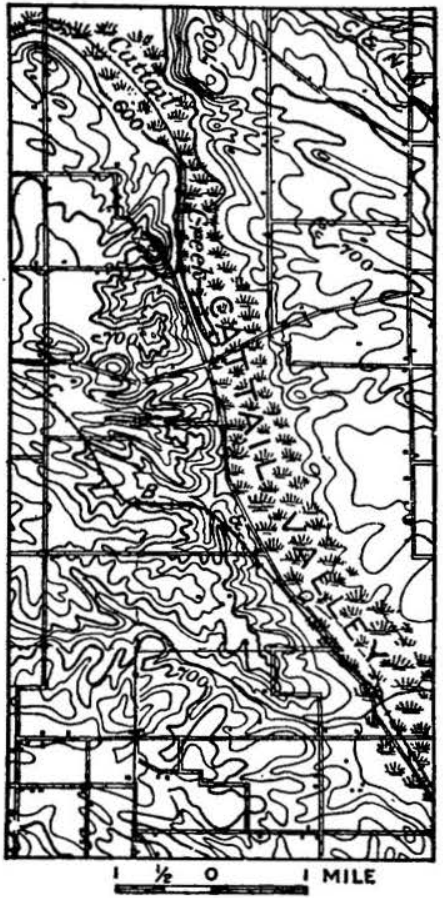

Figure c.-Map of Cattail Valley, Whiteside County, Ill. decomposed. Analysis 310 (p. 27) represents the composition of peat taken at several points along the sides of a large drainage ditch that crosses the deposit. It shows the peat to be similar in composition and quality to that which occurs in the southern part of Cattail Slough. The ash content is 16.63 per cent, the calorific value is 8,464 British thermal units, and the nitrogen content is 2.52 per cent. Analyses 311 and 312 (p. 27) show the composition of the muck in this locality. 
INDIANA.

\section{GENERAI FEATURES.}

The peat deposits of Indiana are confined to the glaciated region in the northern part of the State. The greater part of the peat occupies the basins of ancient glacial lakes, but a few deposits were formed in depressions between sand dunes and in marshes along meandering streams. The following counties contain most of the peat in Indiana: Allen, Dekalb, Elkhart, Fulton, Jasper, Kosciusko, Lagrange, Lake, Laporte, Marshall, Newton, Noble, Porter, Pulaski, St. Joseph, Starke, Steuben, Wabash, and Whitley. Areas of peat in these counties are shown on maps accompanying a report ${ }^{29}$ published by the Indiana Department of Geology and Natural Resources. Bogs and marshes containing sphagnum and grass-sedge peat are the predominant types. The deposits range from 5 to 2,500 acres in area. and from 1 to 25 feet in thickness, but most of them are less than 100 acres in area and less than 6 feet in depth. The upper layers are generally brown and fibrous and the lower black, well-decomposed; and plastic. A large quantity of the peat is underlain by marl of good quality.

The peat deposits of Indiana are estimated to be capable of yielding 51,000,000 short tons of air-dried peat. This estimate, which is based upon Taylor's report, ${ }^{30}$ supplemented by data collected by R. G. Butler, includes only peat and muck deposits that are 20 acres or more in area and not less than $2 \frac{1}{2}$ feet deep, except that if small deposits occur in the same general locality they have been considered as a unit. The large, shallow deposits are included because they offer great agricultural possibilities. Probably not more than one-fourth of the peat in this estimate, or about $13,000,000$ tons, is suitable for' commercial use other than as crop soil. The deep impure peat can not be used for fuel, but much of it is high in nitrogen and may be valuable for fertilizer.

Quantity of peat and muck in Indiana, by counties, estimated as air-dried material (short tons).

Allen 500,000

Dekalb 1, 612,000

Elkhart $3,500,000$

Fulton 1, 500,000

Jasper $2,250,000$

Kosciusko 6, 000,000

Lagrange 2, 000,000

Lake $3,350,000$

Laporte $1,660,000$

Taylor A. E., The peat deposits of northern Indiana: Indiana Dept. Geology and Nat. Res., Thirty-first Ann. Rept., pp. 73-285, 1906.

${ }^{30}$ Taylor, A. E., op. cit. 


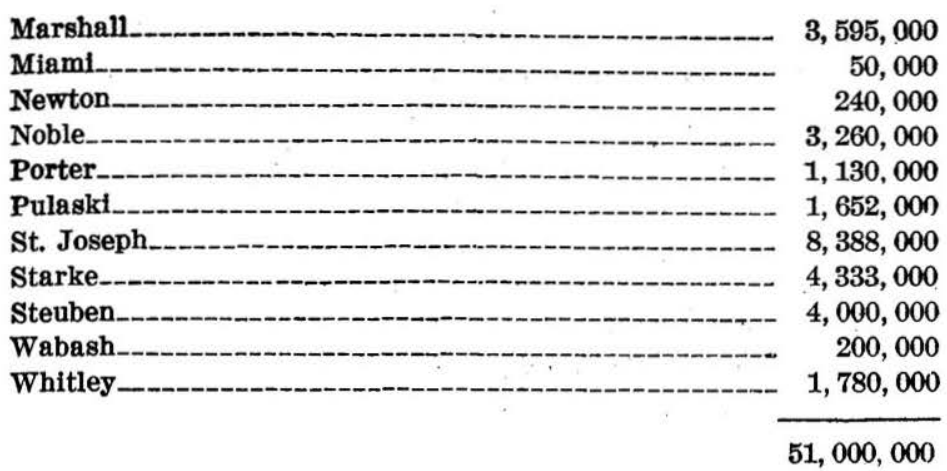

Many peat deposits in Indiana have been described in detail by Taylor in the Thirty-first Annual Report of the Indiana Department of Geology and Natural Resources. The following additional deposits were examined by the United States Geological Survey:

\section{AIIEN OOUNTY.}

Locality 1.-A deposit in the SW. $\frac{1}{4}$ sec. 25 and the SE. $\frac{1}{4}$ sec. 24 , Aboite Township, about 8 miles west of Fort Wayne, which is owned by the Virginia-Carolina Chemical Co., of Richmond, Va., is about 100 acres in area. The peat is brown and fibrous and is composed chiefly of the remains of grasses and sedges. The deposit is 4 feet in average depth and should yield approximately 80,000 tons of air-dried peat. The dominant living vegetation consists of cat-tails, giant sedges, and swamp willows. Samples were taken from eight test borings made at intervals of 100 yards along a north-south line through the center of the deposit. Two typical samples (analyses 339 and 340, p. 27) were obtained by mixing peat from the different test holes. A comparison of these analyses shows the peat to be uniform in composition. The average ash content is about 18.4 per cent and the average nitrogen content about 3.3 per cent. Although the ash content is not high, the calorific value is rather low and the peat is too fibrous for good fuel. The Virginia-Carolina Chemical Co. operated a fertilizer plant at this deposit until its buildings were destroyed by fire in April, 1918. The machinery was saved, and the company reports that the plant will resume operations. Analysis 338 (p. 27) shows the quality of the dried peat from this deposit as used commercially for fertilizer.

DERALB COUNTY.

Locality 2.-A deposit in the NE. $\frac{1}{4}$ sec. 9, Butler Township, is the site of the peat-moss plant of Baker \& Co., which is no longer in operation. The deposit is 40 acres in area and 12 feet in average 
thickness and should yield approximately 96,000 short tons of air. dried peat. The living vegetation consists chiefly of grasses and the huckleberry. The peat is dark brown and rather friable near the surface; the underlying peat is plastic. Four test borings were made along a line from east to west across the deposit and a representative sample (analysis 343, p. 27 ) was obtained by mixing peat taken from each of these test holes. The ash content is 5.34 per cent, and the calorific value 8,757 British thermal units. The peat therefore appears to be suitable for fuel.

Locality 3.-A deposit in the NW. $\frac{1}{4}$ sec. 33 and the NE. $\frac{1}{4}$ sec. 32 , Richland Township, which is about 30 acres in area and 17 feet in average depth, should yield approximately 102,000 short tons of airdried peat. The peat ranges in color from light to dark brown and in texture from fibrous to plastic. It is composed chiefly of the remains of grasses and sedges, and plants of this kind are still the, predominant living vegetation of the area. Peat is still in process of formation on this deposit. Analysis 341 (p. 27) shows the quality of the peat of this deposit. The ash content is 12.08 per cent, the nitrogen content 3 per cent, and the calorific value 8,412 British thermal units. The deposit, though rather small in area, seems to contain peat suitable for either fuel or fertilizer.

\section{ELKHART COUNTY.}

Locality 4.-A deposit in the SE. $\frac{1}{4}$ sec. 10, T. 36 N., R. 6 E., is about 30 acres in area and 4 feet in average depth. It is estimated to centain the equivalent of 24,000 tons of air-dried peat. The peat is dark brown and well decomposed and consists chiefly of the remains of mosses, sedges, and grasses. Four test borings were made and a representative sample (analysis 332, p. 27) was obtained by mixing peat taken from each of these holes. The ash content (25.03 per cent) is too high for the peat to be used as fuel. However, the nitrogen content ( 2.84 per cent) is relatively high, and the deposit is therefore valuable as a source of humus or nitrogen or as a crop soil.

Locality 5.-A large deposit in the E. $\frac{1}{2}$ sec. 27, the SE. $\frac{1}{4}$ sec. 26, the center of sec. 35 , and the SE. $\frac{1}{4}$ sec. 36 , T. 35 N., R. 5 E., contains an area of about 700 acres, and the average depth of the peat is about 18 feet. The deposit should therefore yield approximately $2,500,000$ short tons of air-dried peat. The peat, which is composed chiefly of the remains of moss and grass, is brown and fibrous and contains considerable woody material. The deposit is distinctly stratified and is underlain by sand. The living vegetation consists largely of grasses. However, scattered groves of maple, willow, and poplar are found in places in the area. Four test borings were made, at intervals of 400 yards along a northwest-southeast line through the center of the deposit, and a typical sample (analysis 333, p. 27) 
was obtained by mixing peat taken at intervals of 2 feet in depth in each of these test holes. The peat appears to be of good quality for fuel or for a nitrogenous ingredient of commercial fertilizer.

Locality 6.-A deposit in the N. $\frac{1}{2}$ sec. 13 and in sec. 14, Osolo Township, and the NW. $\frac{1}{4}$ sec. 18, Washington Township, is about 500 acres in area. The peat is dark brown and fibrous and is composed chiefly of the remains of mosses, grasses, and sedges. Some woody matter was also found. Four test borings were made at intervals of 400 yards along an east-west line through the center of the deposit. About 350 acres contains peat 12 feet in average depth, and the peat in the remaining 150 acres is about 4 feet in average thickness. The deposit should yield approximately 960,000 short tons of air-dried peat. A representative specimen (see analysis 337, p. 27) was obtained by mixing peat taken at intervals of 2 feet in depth in each of the test holes. The ash content (10.99 per cent) is relatively low and the fuel value $(8,566$ British thermal units) comparatively high. The peat is chemically suitable for fuel, but it is probably too fibrous to be utilized for the manufacture of machine peat. A noteworthy feature of the analysis is the high nitrogen content (3.29 per cent). The peat seems to be well adapted for making fertilizer.

FULTON COUNTY.

Locality \%.-A deposit in sec. 20 , T. 30 N., R 5 E., about 2 miles east of Akron, occupies an area of about 300 acres. The peat occurs in pockets and hence is not uniform in depth. The average thickness is probably about 10 feet, so that the deposit should yield approximately 600,000 short tons of air-dried peat. The surface is well drained, although the general elevation of the deposit is not far above the water level in several near-by lakes. The living vegetation is composed chiefly of grasses and of aspen, poplar, and willow trees. Many fallen logs lie on the surface. The peat is brown and fibrous and contains much woody matter. The surface layer is unusually fibrous and might be advantageously utilized for packing material or stable litter. Analysis 328 (p. 27) shows the quality of the peat. The ash content is 10.99 per cent and the fuel value is 8,888 British thermal units. Although the analysis indicates that the peat is suitable for fuel, it is so fibrous that there is doubt whether the deposit could be used in the manufacture of machine fuel. The high nitrogen content (2.98 per cent) indicates that the peat may be of agricultural value.

Locality 8.-A bog in the SE. $\frac{1}{4}$ sec. 21 and the NE. $\frac{1}{4}$ sec. 28 , T. 30 N., R. 5 E., about 3 miles east of Akron, is about 38 acres in area and 8 feet in average thickness and is estimated to be capable of yielding 600,000 short tons of air-dried peat. The peat, which is composed chiefly of the remains of mosses, grasses, and shrubs, is brown and 
fibrous to plastic and contains some woody matter. The living vege. tation consists largely of the cranberry, tamarack trees, and swamp ferns. A composite sample (analysis 329, p. 27) was obtained by mixing peat from various test holes. The ash content is only 8.82 per cent, and the nitrogen content is above the average. The peat therefore seems to be of good quality for fuel or fertilizer.

\section{JASPER OOUNTY.}

Locality 9.-A large deposit occupies parts of secs. 7, 8, 12, 13, 14, 16,17 , and 18 , Tps. 30 and 31 N., R. 5 W. The area sampled consists of about 450 acres in sec. 18 . The average thickness of the deposit in sec. 18 is about 10 feet and the quantity of air-dried peat available is about 900,000 short tons. The peat is largely dark brown and fibrous to plastic. Greenish pond peat underlain by clay and sand was found near the bottom. The peat is composed chiefly of the remains of sedges and of grasses. The dominant living vegetation in sec. 18 is grasses and sedges. A representative sample (analysis 321, p. 27) was obtained by mixing peat taken at intervals of 2 feet in depth from each of the test holes put down along an east-west line through the center of sec. 18 . The peat contains 3.34 per cent nitrogen and 16.15 per cent of ash, and the calorific value is 8,477 British thermal units. Although the deposit is not easily accessible, it would supply a large quantity of peat suitable for agricultural uses or for fuel.

\section{KOSCIUSKO COUNTY.}

Locality 10.-A large bog occupies parts of secs. 12, 13, 14, 23, 24, 25, and 26, Etna Township, and parts of secs. 18, 19, 30, and 31, Prairie Township. The area of the bog is about 2,500 acres, and the average thickness of the peat is 10 feet. The deposit, according to estimate, would yield $5,000,000$ short tons of air-dried peat. The living vegetation consists chiefly of grasses, sedges, sphagnum, swamp ferns, and tamarack. Many fallen tamarack logs and stumps are found beneath the peat, as well as on the surface. The peat is brown and grades from fibrous material in the upper layers to plastic peat at the bottom. Test borings were made along an east-west line across the centers of sec. 13, Etna Township, and sec. 18, Prairie Township. Typical samples (analyses 330 and 331, p. 27) were obtained by mixing peat taken at intervals of 2 feet in depth from each of the test holes. The peat is uniform in quality. The nitrogen content is about 2.5 per cent and the ash content about 9 per cent. The average calorific value of the two samples is 8,497 British thermal units. This is one of the largest peat bogs in Indiana and contains a large quantity of peat suitable for either fuel or fertilizer. The deposit adjoins the tracks of the Pennsylvania Railroad and is therefore readily accessible. 
Locality 11.-A bog that occupies an old lake basin in secs. 7, 8, and 18, German Township, is about 300 acres in area, 10 feet in average thickness, and, according to estimate, would yield 600,000 short tons of air-dried peat. The peat, which is composed chiefly of the remains of sphagnum moss and grasses, is light to dark brown, fibrous near the surface, and plastic at the bottom. The living vegetation consists chiefly of grasses, sedges, sphagnum, the huckleberry, and poplar, and maple trees. A composite sample (analysis 325, p. 27) was obtained by mixing peat taken at intervals of 2 feet in depth from numerous test borings. The deposit could easily be drained and worked to a depth of 6 or 7 feet, and the peat appears to be of good quality for either fuel or fertilizer.

Locality 12.-A small bog in the NE. $\frac{1}{4}$ sec. 10 , near Tyner, which is 20 acres in area and contains a deposit of peat 15 feet in average thickness, should yield approximately 60,000 short tons of air-dried peat. The peat is dark-brown and is composed largely of the remains of sphagnum moss. It is fibrous and mossy except in the lower layers. The living vegetation consists chiefly of the blackberry, grasses, and trees. The peat is underlain by clay, marl, and sand. A representative sample (analysis 326, p. 27) was obtained by mixing peat from several test holes put down along an east-west line through the center. The peat seems to be of good quality for fuel. Analysis 327 (p. $2 \pi$ ) shows the quality of a sample of air-dried hand-cut peat from this bog. The ash content of this sample is 8.17 per cent and the calorific value 9,299 British thermal units. Hand-cut peat from this bog has been used for fuel by C. F. Brown, the owner.

\section{NOBLE COUNTY.}

Locality 13.-A bog in the NE. $\frac{1}{4}$ sec. 29 , and the NW. $\frac{1}{4}$ sec. 28 , Wayne Township, is about 100 acres in area and contains peat 3 feet in average thickness. The peat is brown and rather fibrous and is composed chiefly of the remains of mosses and sedges. The living vegetation consists of moss, willow, sumac, and tamarack. A typical sample (analysis 342, p. 27 ) was obtained by mixing peat taken from several test holes. The peat appears to be of good quality for fuel or fertilizer. The deposit, however, is so shallow that it can not be recommended as a favorable site for a peat plant.

\section{PULASKI OOUNTY.}

Locality 14.-A large deposit that occupies secs. 7, 8, and 9 and parts of 16 and 17, Rich Grove Township, is about 1,000 acres in area, and the peat is 4 feet in average depth. The deposit should yield approximately 800,000 short tons of air-dried peat. The peat, which is composed of the remains of grasses and sedges, is dark brown near 
the top and yellow to green near the bottom and is underlain by green and blue clay. The living vegetation consists chiefly of grasses and sedges. The deposit has been drained, and much of it is under culti. vation, corn being the principal crop grown. A typical sample (analysis 322, p. 27) was obtained by mixing peat taken from numerous test holes. The nitrogen content is 2.91 per cent. The area is probably of more value for cultivation than as a source of peat fuel.

ST. JOSEPH COUNTY.

Locality 15.-A deposit in the NE. $\frac{1}{4}$ sec. 12 and the E. $\frac{1}{2}$ sec. 1 , Warren Township, is 250 acres in area, and the peat, which occurs in pockets, is about 6 feet in average depth. It is estimated that this deposit should yield 240,000 short tons of air-dried peat. The. peat is brown to black and fibrous and is composed chiefly of sedges, grasses, and moss. The living vegetation consists chiefly of marsh grass. A composite sample (analysis 324, p. 27) was obtained by mixing peat taken from several test holes. The peat is low in ash, the nitrogen content is 2.86 per cent, and the calorific value is 8,331 British thermal units. An odor of hydrogen sulphide was noted while making the test borings.

Locality 15a.-A deposit in secs. 18, 19, and 20, T. 37 N., Olive Township, which is well drained, was formerly the northern part of the Kankakee Valley marsh. The deposit consists of muck, which is shallow and contains a large proportion of inorganic mineral matter. Most of the deposit is under cultivation and is used extensively for growing mint. As the muck is of no value for fuel or as an ingredient of commercial fertilizer, no samples were taken for analysis.

Locality 15b.-A deposit of muck in secs. 15 and 16, Portage Township, is similar in depth and quality to that in locality 15a. It is used for the cultivation of various crops and is more valuable as crop soil than as a source of fuel. No samples were taken for analysis.

Locality 16.-A bog that occupies parts of secs. 28 , 33, and 34 , T. 36 N., R. 2 E., and parts of secs. $2 ; 3,11,12,13$, and 14, T. 35 N., R. 2 E., is one of the largest peat deposits in Indiana. The plant of the St. Joseph Humus Co., of Lakeville, is in the SE. $\frac{1}{4}$ sec. 34. The peat is brown and fibrous to plastic and consists largely of the remains of grasses, sedges, sphagnum, and tamarack. The living vegetation consists largely of the same plants. The average thickness is about $6 \frac{1}{2}$ feet. Analyses 334, 335, and 336 (p. 27) show the quality of the raw peat from this deposit. The peat contains a large quantity of ash, but the nitrogen content ranges from 2.20 to 2.92 per cent. The equipment at the plant of the St. Joseph Humus Co. consists of excavating, loading, and grinding machinery 
and one large rotary drier. The product of this plant is used as a nitrogenous ingredient of commercial, fertilizers.

BTAREE COUITTY.

Locality 17.-A deposit in the SE. $\frac{1}{4}$ sec. 21 , the SW. $\frac{1}{4}$ sec. 22 , the W. $\frac{1}{2}$ sec. 27 , sec. 28 , the NE. $\frac{1}{4}$ sec. 33 , and the NW. $\frac{1}{4}$ sec. 34 , Wayne Township, is about 300 acres in area, and the peat is 8 feet in average thickness. The deposit should yield approximately 480,000 short tons of air-dried peat. The living vegetation consists chiefly of sedges, grasses, and the swamp willow. The peat, which is made up chiefly of the remains of sedges and grasses, is dark brown to light brown, fibrous near the top, soft and decomposed at the bottom, and is underlain by sand. Test borings were made at intervals of 450 yards along a northwest-southeast line across the deposit. Two composite samples (analyses 318 and 319, p. 27) were obtained by mixing peat taken at intervals of 2 feet in depth from each of the test holes. The nitrogen content is high, and the peat seems to be of good quality for fuel or for a nitrogenous ingredient of commercial fertilizers.

Locality 18.-Deposits in secs. 31,32 , and 33, T. 32 N., R. 3 W., Wayne Township, are about 600 acres in area and 6 feet in average thickness and should yield approximately 720,000 short tons of airdried peat. The living vegetation consists chiefly of sedges and grasses. Large quantities of marsh grass are produced from this deposit and used for packing material. The peat, which is brown and fibrous, is composed chiefly of the remains of grasses and sedges. A typical sample (analysis 320) was obtained by mixing peat taken at intervals along a large drainage ditch that crosses the marsh. Although the ash content is only 12.1 per cent, the peat is fibrous and is therefore not well adapted for machine fuel. The high nitrogen content ( 3.26 per cent) indicates that the peat may be of value as a nitrogenous ingredient of commercial fertilizers. If properly drained a large part of the area might be utilized for the cultivation of crops.

Locality 19.-A deposit in the SW. 1 sec. 10, T. 32 N., R. 3 W., Wayne Township, is about 70 acres in area and 7 feet in average thickness; it should yield approximately 98,000 short tons of airdried peat. The peat, which is composed chiefly of the remains of moss, sedges, and grasses, is brown and fibrous near the top but soft and plastic at the bottom. The living vegetation includes a thick growth of grasses, sedges, and the cranberry. A typical sample (analysis 323, p. 27) was obtained by mixing peat taken at intervals of 2 feet in depth from several test holes put down along a northsouth line through the center of the deposit. The peat is uniform in texture and is physically suitable for machine-peat fuel. The ash content is 14.04 per cent.

$$
\text { 91065 }-22-9
$$


\$TEUBEN DOUNTY.

Locality 20.-A large bog that occupies parts of secs. 15, 16, 21, 22 , and 28 , Steuben Township, contains a deposit about 600 acres in area and 14 feet in average thickness, which should yield approximately 1,680,000 short tons of air-dried peat. The living vegetation consists chiefly of sedges, grasses, moss, and small tamarack trees. The peat is light brown and fibrous near the surface, but the under. lying layers are dark brown, soft, plastic, and well disintegrated and are composed chiefly of the remains of sedges, grasses, and moss. Test borings were made at intervals of 100 yards along a north-south line extending through the center of the deposit. Analysis 344 (p. 27) shows the peat to be of excellent quality for fuel or fertilizer. The ash content is 12.44 per cent, the percentage of nitrogen 3.66, and the calorific value 8,782 British thermal units. There are many other peat deposits in this locality, but this one is among the best in Steuben County and would be a good site for a peat plant.

Locality 21.-A bog that surrqunds Little Center Lake, near the town of Angola, Pleasant Township, contains a deposit about 40 acres in area and 4 feet in average thickness. The peat is underlain by clay and sand. The living vegetation consists chiefly of grasses, sedges, moss, and tamarack. The peat is brown and fibrous and contains many roots of trees and shrubs. A typical sample (analysis 345, p. 27) was obtained by mixing peat taken from several test holes put down at different places throughout the deposit. The peat appears to be of good quality for fuel or fertilizer, but the deposit is probably too small for the production of peat on a commercial scale.

Locality 22.-A bog that surrounds Big Center Lake, occupying parts of secs. 21 and 22, Pleasant Township, contains a deposit about 150 acres in area and 10 feet in average thickness, which should yield approximately 300,000 short tons of air-dried peat. The peat, which is made up chiefly of the remains of sphagnum moss, is brown and fibrous near the top of the deposit but soft and well decomposed at the bottom. The living vegetation consists chiefly of sphagnum and tamarack. Analysis 346 (p. 27) shows the peat to be apparently of good quality for fuel or fertilizer. The calorific value is 8,922 British thermal units and the nitrogen content is 3.76 per cent.

онIо.

The principal peat deposits in Ohio occur in the region bordering Lake Erie and in the northwestern part of the State. (See Pl. X.) The following counties contain the largest deposits: Ashland, Ashtabula, Auglaize, Crawford, Defiance, Geauga, Hardin, Huron, Logan, Mahoning, Mercer, Portage, Seneca, Stark, Summit, Trumbull, Van Wert, Wayne, Williams, and Wyandot. As in other States in the 


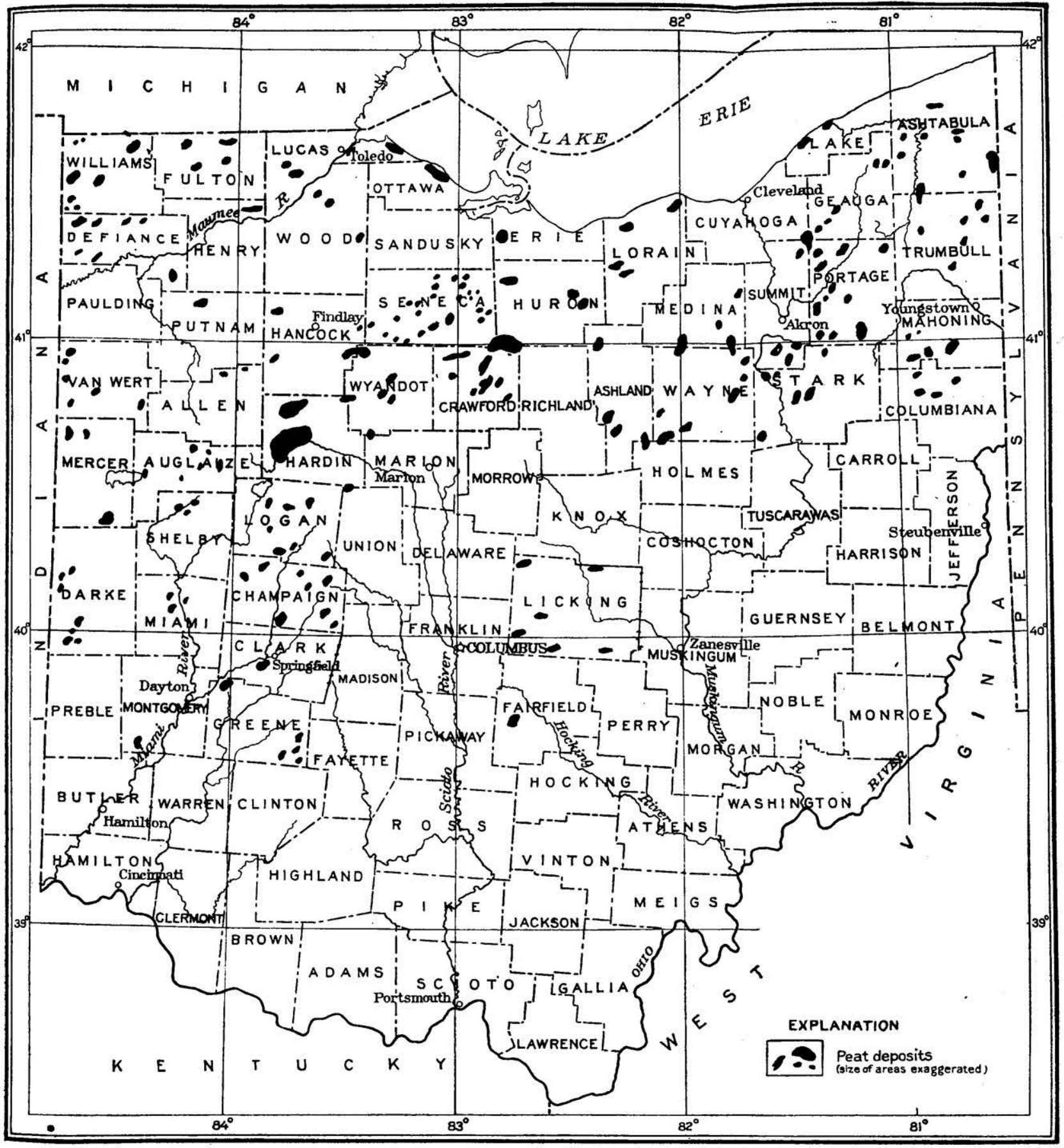

25

25

50 MILES

MAP SHOWING DISTRIBUTION OF PEAT IN OHIO. 

northern region, most of the peat deposits of Ohio were formed in glacial lakes and ponds ind in other wet places in depression of glacial origin. In some places peat entirely surrounds small ponds, the remnants of larger glacial lakes that have been gradually filled by vegetation. In other places peat has accumulated on relatively flat, undrained land. Dachnowski ${ }^{31}$ states that the following varieties of peat occur in Ohio: Coarse, fibrous, spongy, light-brown peat in the upper layers of basin deposits; compact, well decomposed, plastic dark-brown to black peat in the lower layers of basin deposits; fibrous, firm brown peat in built-up deposits.

Most of the peat in Ohio was formed chiefly by the decay of sphagnum, grasses, and sedges and is therefore largely fibrous near the surface. Many of the deposits are underlain by marl. The area of peat and muck in Ohio is estimated to be about 150,000 acres. Perhaps one-third of this area, or 50,000 acres, contains workable peat. The thickness of the peat ranges from 1 to 20 feet, and the average is about 5 feet. If these figures are correct the peat deposits of Ohio should yield approximately $50,000,000$ short tons of airdried peat. Probably 25,000,000 tons is suitable for fuel or fertilizer.

The following table ${ }^{82}$ briefly describes the principal peat deposits in Ohio:

Location, area, depth, and character of peat deposits in Ohio.

\begin{tabular}{|c|c|c|c|c|c|c|}
\hline $\begin{array}{l}\text { Deposit } \\
\text { No. }\end{array}$ & County. & Township. & Section. & $\begin{array}{l}\text { Area } \\
\text { (acres). }\end{array}$ & $\begin{array}{l}\text { Depth } \\
\text { (feet). }\end{array}$ & Character of peat and vegetation. \\
\hline 1 & Ashland. & Lake... & 23 & 60 & 17 & Reddish brown, flbrous. Cranberry- \\
\hline 2 & Ashtabula. . & Orwell.. & & 1,000 & 5 & $\begin{array}{l}\text { Dark brown, partly fibrous, woody and } \\
\text { well decayed. Tamarack, maple, } \\
\text { alder. }\end{array}$ \\
\hline 3 & Champaign.. & Urbana... & 31-32 & 600 & 4 & Dark brown, slightly fibrous, well de- \\
\hline 4 & Columbians. & Center... & $7-8$ & 200 & 6 & Dark brown, well decayed, partly \\
\hline 5 & Crawford.. & Auburn... & $\cdots$ & 500 & 5 & $\begin{array}{l}\text { Dark brown, partly flbrous. Under } \\
\text { cultivation. }\end{array}$ \\
\hline $\begin{array}{l}6 \\
7\end{array}$ & Cuyahoga. & $\begin{array}{l}\text { Cranberry. } \\
\text { Solon..... }\end{array}$ & $24-26$ & $\begin{array}{r}300 \\
300-400\end{array}$ & $\begin{array}{r}6 \\
10\end{array}$ & Same. \\
\hline 8 & Darke.... & Butler.... & 7,18 & $200(?)$ & 2 & $\begin{array}{l}\text { Dark brown, well decayed. Under } \\
\text { cultivation. }\end{array}$ \\
\hline $\begin{array}{r}9 \\
10\end{array}$ & Deflance... & $\begin{array}{l}\text { Harrison.. } \\
\text { Milford... }\end{array}$ & 2-11 & 100 & $\begin{array}{r}4 \\
15\end{array}$ & $\begin{array}{l}\text { Same, slightly fibrous. } \\
\text { Dark brown, well decayed, slightly }\end{array}$ \\
\hline 11 & ....do... & .....do. & & 5 & 15 & $\begin{array}{l}\text { Brown, flbrous, partly structureless. } \\
\text { Under cultivation. }\end{array}$ \\
\hline 12 & Fairfield. & Amanda. & 26 & $\cdots . . .$. & 12 & Dark brown, well decayed, partly \\
\hline 13 & Geauga. & Bainbridge & & 70 & 19 & $\begin{array}{l}\text { Brown, partly fibrous, well decsyed } \\
\text { with severalbog associations. }\end{array}$ \\
\hline $\begin{array}{l}14 \\
15\end{array}$ & Gr..do... & $\begin{array}{l}\text { Burton... } \\
\text { Bath..... }\end{array}$ & 10 & $\begin{array}{l}100 \\
100(?)\end{array}$ & $\begin{array}{r}17 \\
7\end{array}$ & $\begin{array}{l}\text { Same, slightly woody. } \\
\text { Dark brown, partly fibrous. Under }\end{array}$ \\
\hline 16 & Hancock.... & Big Lick... & $20-34$ & 2,000 & 3 & Dark brown, partly structureless, some- \\
\hline 17 & Hardin. & Marion.. & 27 & 16,000 & 5 & $\begin{array}{l}\text { Dark brown, partly flbrous, well de- } \\
\text { composed. Under cultivation. }\end{array}$ \\
\hline
\end{tabular}

a Dachnowski, Alfred, The peat deposits of Ohio: Ohio Geol. Survey Bull, 16, 4th ser., p. $18,1912$.

Idem, pp. 363-365. 
Location, area, depth, and character of peat deposits in Qhio-Continued.

\begin{tabular}{|c|c|c|c|c|c|c|}
\hline $\begin{array}{l}\text { Deposit } \\
\text { No. }\end{array}$ & County. & Township. & Section. & $\begin{array}{c}\text { Ares } \\
\text { (acres). }\end{array}$ & $\begin{array}{l}\text { Depth } \\
\text { (feet). }\end{array}$ & Character of peat and vegetation. \\
\hline $\begin{array}{l}18 \\
19\end{array}$ & $\begin{array}{l}\text { Hardin..... } \\
\text { Holmes... }\end{array}$ & $\begin{array}{l}\text { Marion ....... } \\
\text { Washington.. }\end{array}$ & $\begin{array}{l}27 \\
26\end{array}$ & 2 & 19 & $\begin{array}{l}\text { Dark brown; well decomposed. Maple, } \\
\text { Brown, fibrous, well decomposed below }\end{array}$ \\
\hline 20 & Huron.. & Richmond & & 5,500 & 15 & Dark brown, partly well decomposed. \\
\hline 21 & ......do. & New Haven. & & 3,500 & $\boldsymbol{\theta}$ & $\begin{array}{l}\text { Dark brown, well decomposed. Under } \\
\text { cultivation. }\end{array}$ \\
\hline $\begin{array}{l}22 \\
23\end{array}$ & Licking. & $\begin{array}{l}\text { Licking.. } \\
\text {.....do... }\end{array}$ & & 4,000 & $\begin{array}{l}30 \\
30\end{array}$ & $\begin{array}{l}\text { Dark brown to black, partly fibrous. } \\
\text { Dark brown, well decomposed, partly } \\
\text { flbrous. }\end{array}$ \\
\hline 24 & .....do.. & do & & & 30 & $\begin{array}{l}\text { Brawn, ooarsely flbrous. Cranberry. } \\
\text { sphagnum meadow. }\end{array}$ \\
\hline 25 & Mahoning & Besver ... & $24-36$ & 500 & 16 & $\begin{array}{l}\text { Dark brown well decomposed, partly } \\
\text { flbrous. Tamarack. }\end{array}$ \\
\hline 26 & Medina. & Harrisville. & & 2,000 & $\ldots 5$ & $\begin{array}{l}\text { Dark brown, partly fibrous. Under } \\
\text { cultivation. }\end{array}$ \\
\hline 27 & ......do. & Westfield. & & & 8 & $\begin{array}{l}\text { Fibrous, partly well decomposed. } \\
\text { Sterile soll. }\end{array}$ \\
\hline 28 & Mercer. & Granville & & 1,000 & 7 & $\begin{array}{l}\text { Dark brown; structureless, slightly } \\
\text { flbrous. Under cultivation. }\end{array}$ \\
\hline $\begin{array}{l}29 \\
30 \\
31\end{array}$ & Montgomery & $\begin{array}{l}\text {....do..... } \\
\text { G̈.do..... }\end{array}$ & $\begin{array}{l}35 \\
18\end{array}$ & & $\begin{array}{r}4 \\
1-2 \\
4\end{array}$ & $\begin{array}{l}\text { Same. } \\
\text { Same. } \\
\text { Dark brown, well decomposed. Fossil }\end{array}$ \\
\hline 32 & Portage. & Atwater. & & 400 & 3 & $\begin{array}{l}\text { Dark brown, slightly flbrous. Tams. } \\
\text { rack, maple. }\end{array}$ \\
\hline 33 & ....do.. & Brimfield. & 20 & 200 & 18 & $\begin{array}{l}\text { Dark brown, partly fibrous and woody. } \\
\text { Maple, ash, elm. }\end{array}$ \\
\hline 34 & do & .....do. & & 2 & 9 & $\begin{array}{l}\text { Brown, fbrous. Cranberry-sphagnum } \\
\text { meadow. }\end{array}$ \\
\hline 35 & & Franklin. & & 30 & $.10-15$ & $\begin{array}{l}\text { Dark brown, well decomposed. Tamer } \\
\text { rack muskeg. }\end{array}$ \\
\hline 36 & ....do. & Mantus... & & 2,000 & 8 & $\begin{array}{l}\text { Dark brown, well decomposed, partly } \\
\text { silty. Maple, ash, elm. }\end{array}$ \\
\hline $\begin{array}{l}37 \\
38\end{array}$ & $\begin{array}{l}\ldots . . \text { do } \\
\ldots . . \text { do }\end{array}$ & $\begin{array}{l}\text { Nelson.... } \\
\text { Ravenna. }\end{array}$ & & $100(?)$ & $\begin{array}{r}4 \\
17\end{array}$ & $\begin{array}{l}\text { Nearly black and silty } \\
\text { Brown, fibrous, partiy well decom. }\end{array}$ \\
\hline 39 & Seneca.. & Big Spring & 32 & $3,000(?)$ & 8 & $\begin{array}{l}\text { posed. Seviral associstions. } \\
\text { Dark brown, almost structureless. } \\
\text { Partly under cultivation. }\end{array}$ \\
\hline $\begin{array}{l}40 \\
41\end{array}$ & $\begin{array}{l}\text { Shelby... } \\
\text { Stark... }\end{array}$ & $\begin{array}{l}\text { Van Burer } \\
\text { Canton... }\end{array}$ & $\begin{array}{r}14 \\
6-7\end{array}$ & $100(?)$ & $\begin{array}{l}12 \\
19\end{array}$ & $\begin{array}{l}\text { Same. } \\
\text { Brown, flbrous, partly well decom } \\
\text { posed. Under cultivation. }\end{array}$ \\
\hline $\begin{array}{l}42 \\
43 \\
44\end{array}$ & $\begin{array}{l}\text {....do... } \\
\text { summit. }\end{array}$ & Copley... & & 3,000 & $\begin{array}{r}5 \\
5 \\
14\end{array}$ & $\begin{array}{l}\text { Same. Tamarack. } \\
\text { Same, partly under cultivation. } \\
\text { Dark brown, moderately flbrous. }\end{array}$ \\
\hline $\begin{array}{l}45 \\
46\end{array}$ & l...do. & $\begin{array}{l}\text { Portage.. } \\
\text { Hudson.. }\end{array}$ & & 2,500 & $\begin{array}{l}17 \\
18\end{array}$ & $\begin{array}{l}\text { Partly under cultivation. } \\
\text { Same. Tamarack. } \\
\text { Dark brown, well decomposed. Maple, }\end{array}$ \\
\hline 47 & Trumbull & Greene... & & 1,500 & 5 & $\begin{array}{l}\text { Dark brown, fibrous, partly well de- } \\
\text { composed. Tamarack. }\end{array}$ \\
\hline 48 & Wayne. & Baughman. & & $300(?)$ & 11 & $\begin{array}{l}\text { Brown, flbrous, partly structureless. } \\
\text { Under cultivation. }\end{array}$ \\
\hline 49 & ......do. & .....do. & & & 11 & $\begin{array}{l}\text { Reddish brown, fibrous. Under culti- } \\
\text { vgtion. }\end{array}$ \\
\hline 50 & do & ......do.. & & & 11 & $\begin{array}{l}\text { Nearly black, well decomposed. Under } \\
\text { cultivation. }\end{array}$ \\
\hline 51 & .....do. & .....do. & $1-12$ & 400 & 12 & $\begin{array}{l}\text { Brown, moderately fibrous. Tama } \\
\text { rack. }\end{array}$ \\
\hline 52 & do & .....do. & & & 5 & $\begin{array}{l}\text { Dark brown, well decomposed. Partly } \\
\text { under cultivation. }\end{array}$ \\
\hline $\begin{array}{l}53 \\
54 \\
55\end{array}$ & Williams. & $\begin{array}{l}\text { Florence. } \\
\text { Brido.... }\end{array}$ & $14-15$ & $\begin{array}{l}300 \\
\cdots \cdots\end{array}$ & $\begin{array}{r}5 \\
16 \\
4\end{array}$ & $\begin{array}{l}\text { Brown, partly fbrous. Tamarack. } \\
\text { Same, but well decomposed. }\end{array}$ \\
\hline & & Superio & $14-15$ & 200 & $\begin{array}{r}4 \\
15\end{array}$ & $\begin{array}{l}\text { Same. Tamarack bog. } \\
\text { Dark brown, moderately fibrous. Un- } \\
\text { der cultivation. }\end{array}$ \\
\hline 57 & .....do.... & Madison. . & & 40 & 9 & $\begin{array}{l}\text { Brown, fibrous, partly well decom- } \\
\text { posed. Tamarack. }\end{array}$ \\
\hline 58 & Wyandot. & Crawford. & & 3,000 & 12 & $\begin{array}{l}\text { Dark brown, well decomposed, some- } \\
\text { what woody. Under cultivation. }\end{array}$ \\
\hline $\begin{array}{l}59 \\
60 \\
81\end{array}$ & 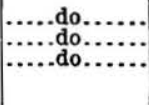 & $\begin{array}{l}\text { (....do... } \\
\text { Crando... }\end{array}$ & & $\begin{array}{r}3,000 \\
3,000 \\
200\end{array}$ & $\begin{array}{r}14 \\
14 \\
8\end{array}$ & $\begin{array}{l}\text { Same. Heath meadow. } \\
\text { Same. Under cultivation. } \\
\text { Dark brown, well decomposed. Under } \\
\text { cultivation. }\end{array}$ \\
\hline
\end{tabular}

Detailed information concerning the distribution and properties of Ohio peats may be obtained by consulting Bulletin 16 of the Ohio Geological Survey. Analyses of peat from Ohio are given on page 48. 
PENARERLANIA.

Most of the peat deposits in Pennsylvanía occupy glacial basins in the northern part of the State. In northeastern Pennsylvania, notably in Monroe, Pike, Sullivan, and adjoining counties, many of the glacial lakes contain small quantities of peat. Ganoga Lake, in Sullivan County, and the lakes in the Pocono district, in Monroe County, are typical of the peat-forming basins of Pennsylvania. There are also some vegetal accumulations in marshes adjoining streams, but this material contains so much silt that it is of little value for fuel. The quantity of peat in Pennsylvania suitable for fuel or fertilizer is probably not more than 1,000,000 short tons of the air-dried material.

\section{COLUMBIA COUNTY.}

Locality 1.-The eastern part of a deposit on the north side of Susquehanna River, about one-fourth of a mile west of the railroad station at Espy, contains about 100 acres of peat whose average thickness is 6 feet. This deposit is long and narrow and contains many patches of dry land. It should yield approximately 120,000 short tons of air-dried peat. The original vegetation, in which deciduous trees predominated, has been removed. The present flora consists chiefly of grasses, sedges, shrubs, and weeds. That the deposit is of filled-basin origin is shown by the marl that underlies it. The upper layers consist of built-up peat. A test hole made near the drying plant of the Espy Humus Fertilizer Co., which is producing peat fertilizer, gave the following results:

Log of test hole near drying plant of Espy Humus Fertilizer Co., Espy, Pa.

Black fibrous peat. Feet.

Chocolate-brown to reddish-brown fibrous peat_._-_._-_-_-_- $1 \frac{1}{2}$

Chocolate-brown to reddish-brown well-decomposed plastic peat

The chemical composition of this peat is shown in the table of analyses (p. 49). The peat from this deposit is excavated by hand, loaded on small tramcars, and hauled by a gasoline motor to the drying plant. The peat is then run through a rotary drier and loaded on railroad cars. As shipped from the plant the peat contains about 10 per cent of moisture and is sold for its nitrogen content.

Locality 2.-Locality 2 includes the western part of the deposit on the north side of Susquehanna River about one-half mile west of the railroad station at Espy. The peat in this locality is separated from that in locality 1 by an intervening area that contains no peat. The deposit ranges from 3 to 10 feet in depth and is underlain by marl about 5 feet deep. Locality 2 is about 60 acres in area and should yield approximately 96,000 short tons of air-dried peat. The 
vegetation is similar to that at locality 1 , and the deposit is quito similar in character. A large area has been cleared. A test hole in the center of this deposit, where peat is being excavated for sale as fertilizer, gave the following results:

Log of test hole in center of deposit of Espy Humus Fertilizer Co., Espy, Pa.

Black fibrous peat.

Brown fibrous peat, containing roots and stems of grasses and sedges

Brown well-decomposed, soft, plastic peat

Brown soft, plastic peat, containing marl

Marl

The chemical composition of this peat is shown in the table of analyses (p. 49). It appears to be suitable for fertilizer, but it contains too much ash for use as fuel.

CRAWFORD COUNTY.

Probably the largest peat deposit in Pennsylvania is in Pymatuning Swamp, western Crawford County. ${ }^{83}$ This swamp is nearly 25 miles long, but peat is not found throughout its area. According to Jennings ${ }^{34}$ this deposit was originally a sphagnum-tamarack bog, but when the tamarack was cut the sphagnum disappeared, and the area was converted into an alder swamp. Two small patches of tamarack yet stand near Hartstown.

PHILADELPHIA OOUNTY.

Locality 3.-A large area surrounded by the Delaware and Schuylkill rivers and Darby Creek, near Hog Island, about 6 miles from the center of the city of Philadelphia, which has been drained and which is largely used for farming and for housing, was carefully examined and numerous test borings were made, but no peat was found in commercial quantities. Most of the surface is covered to a depth of about 1 foot by a mat of fibrous plant remains, consisting of roots and stems of marsh grasses, sedges, weeds, and shrubs. This mat of plant remains is underlain by clayey soil. The area does not contain peat or muck of value for fertilizer.

\section{OTHER COUNTIES.}

Peat is found in the following localities in addition to those already discussed: Marsh near Quakertown, Bucks County; Bear Meadows, Center County; Conneaut Marsh, Crawford County; Cranberry Pond and Presque Isle, Erie County; Log Tavern Ponds, Pike County; and Crane and Tamarack swamps, Wyoming County.

$\$$ Hice, R. R., written communication, 1918.

a Jennings, O. E., written communication. 
NEW YORK.

\section{GENERAI FEATURES.}

The peat deposits of New York, though numerous and widely distributed, may be in general assigned to three divisions-the northern, the west-central, and the southern-and the predominant deposits in each division show differences in manner of formation and in the quality of the peat. According to their manner of formation they may be classified in three groups-filled-basin deposits, built-up deposits, and composite areas. The built-up deposits may be subdivided into fresh-water deposits and salt-marsh deposits.

The peat deposits of northern New York, which includes the Adirondack region, are largely of filled-basin origin and, though numerous and widely distributed, are relatively small. The peat in many places is of great depth, and the upper layers consist chiefly of the remains of sphagnum moss. Many of these deposits are too remote from transportation facilities to be of present economic value. A few built-up deposits and composite areas also occur in this region.

The peat deposits of central and western New York occur in marshes and swamps and are largely of the built-up type. Many of the largest peat areas in the State are in this region, notably the Cicero and Oak Orchard swamps and Montezuma Marsh. Small filled-basin deposits and composite areas, some of which are underlain by marl, also occur in this region.

Peat deposits that may be both fresh-water and salt-marsh deposits occur in southern New York, which includes Long Island. The "drowned lands" of the Wallkill River Valley contain the largest area of peat in the State-a built-up deposit of fresh-water origin-which covers more than 17,000 acres, and the peat in some places is 25 feet deep. The total area of peat in Orange County alone is 40,000 acres. Salt-marsh deposits occur on the shores of Long Island and adjoining parts of Westchester County. The origin of these deposits is similar to that of those on the coast of New England. As the peat is made up of the small variety of plants that tolerate salt water, it is relatively homogeneous but contains so much inorganic matter that it is of little value for fuel.

Newland ${ }^{35}$ estimates that New York contains about 1,250 square miles of peat land, in which the deposits are 3 feet in average thickness. If this estimate is correct, the quantity of air-dried peat available in that State would be about 480,000,000 short tons. Approximately $75,000,000$ tons of this peat is readily accessible.

The following notes contain a description of the deposits sampled for this bulletin. Further detailed data relating to the peat de-

× Newland, D. H., New York State Mus. Bull. 102, p. 19, 1906. 
posits of New York may be found in reports of the New York State Museum. $^{36}$

\section{CAYUGA COUNTY.}

Locality 1.-A deposit west of the Erie Canal locks at Port Byron, Mentz Township, includes several hundred acres, and the peat is reported to have an average thickness of about 4 feet and to be underlain by 6 feet of marl. Onions, celery, and other truck crops are grown on this land, which appears to be more valuable for farming than for the production of peat. No samples were taken.

Montezuma Marsh, one of the largest areas of peat in New York, also lies partly in Cayuga County but is described in the discussions relating to Seneca and Wayne counties.

\section{OLINTON GOUNTY.}

Locality 2.-A swamp along the shore of Lake Champlain at Kings and Catfish bays, about 2 miles south of Rouses Point, Cham! plain Township, which is heavily wooded with maple and elm trees, is covered with muck and impure peat from 6 to 18 inches thick, composed largely of the remains of leaves, twigs, roots, and small pieces of wood. Though usually flooded during the spring and early summer, this area is dry in the fall. Test borings gave the following results :

Thickness of peat and muck in test borings in swamp about 2 miles south of Rouses Point, Clinton County, N. Y.

Feet.

Hole A, south end of swamp: Black sandy muck, underlain by sand.

Hole B, 100 yards from west margin at point midway between north and south ends: Black impure peat containing woody matter

Hole C, north end of swamp: Black muck

Analysis 18 (p. 45), which shows the composition of a specimen taken from hole $B$, indicates that the material may be suitable for fertilizer but is unfitted for fuel.

Locality 3.-A swamp about 2 miles northeast of Plattsburg, extending from the north shore of Cumberland Bay, Lake Champlain, northward to Woodruff Pond and Bay St. Armand, contains a deposit about 500 acres in area and 2 feet in average thickness, which should yield approximately 200,000 short tons of air-dried peat and muck. The living vegetation consists of a large variety of plants, chiefly red maple, elm, swamp alder, tamarack, and scattered pine trees and shrubs, sphagnum moss, cat-tails, sedges, reeds, and other plants.

${ }^{36}$ Ries, H., The uses of peat and its occurrence in New York: New York State Mus, Fifty-fifth Ann. Rept. (State Geologist, Twenty-first Ann. Rept.), pp. r55-r90, Albany, 1901. Parsons, A, L., Peat, its formation, uses, and occurrence in New York: New York State Mus., Fifty-seventh Ann. Rept. (State Geologist, Twenty-third Ann. Rept.), pp. 15-88, Albany, 1903. 
The peat is dark brown and fibrous and contains much woody matter. The lower layers of muck contain a large proportion of sand. Test borings gave the following results:

Thickness of peat in test borings 2 miles northeast of Plattsburg, $N . Y$.

Hole A, north of wagon road, near southeast corner of swamp : Dark-brown woody peat and muck

Feet.

Hole B, south-central part of swamp north of wagon road:

Dark-brown woody, sandy peat and muck

Analysis 19 (p. 45), which was made on a sample taken from hole A, shows the deposit to be of fair quality for fertilizer but wholly unfit for fuel.

Locality 4.-Test holes in a marsh along both shores of Dead Creek, about $1 \frac{1}{2}$ miles north of Plattsburg, in Beekmantown and Plattsburg townships, revealed only a shallow deposit of brown to black silty muck, underlain by blue clay. This marsh occupies the lowlands along Dead Creek, and is subject to annual flooding. Its southern part is classed as locality 8 . No samples were taken for analysis.

\section{DUTCHESS COUNTY.}

Locality 5.-A bog about 1 mile from the village of Fishkill, along the main highway, contains about 300 acres of peat 10 feet in average thickness and should yield approximately 600,000 short tons. A part of this bog is owned by Asa McElhone, of Fishkill, and the remainder by the Orchid Humus Fertilizer Co., of Peekskill. The peat is composite in origin, and the living vegetation is made up principally of maple, alder, and willow trees and cat-tails, reeds, grasses, and sedges. Sphagnum of the large-leafed variety, suitable for surgical dressings, was seen in some places. Many fern hummocks, the roots of which are gathered for orchid humus, occur throughout the bog. The peat, which appears to be of excellent quality for fuel or fertilizer, is dark brown to a depth of about 12 feet and green below this point. Test borings gave the following results:

Log of hole A, in south end of excavation at locality 5,1 mile from Fishkill, N. Y. Feet.

Dark-brown fibrous peat

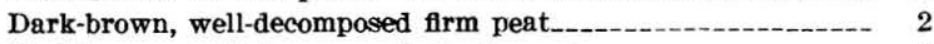

Dark-brown, well-decomposed plastic peat___-_._- 8

Greenish plastic pond peat containing clay 2

In hole $\mathrm{B}$, at the north end of the same excavation, where peat is being produced, the peat is $13 \frac{1}{2}$ feet deep and is similar in texture and color to that in hole A.

Analysis $74^{\prime}$ (p. 45) shows the character of a composite sample obtained by mixing peat taken at intervals of 2 feet to a depth of 
12 feet in each of the two test holes described, and indicates that the peat is of good quality for fuel or fertilizer. Analysis 74a (p. 45) shows the character of a sample taken from a stock pile of peat ex. cavated for fertilizer from the surface layer of the deposit and ex. posed to the weather for a year, and indicates that the peat is of excellent quality for fuel. The low ash content is due to the fact that the samples were taken from the upper layer of the deposit, where no inorganic material had been washed in by flood waters. This deposit contains a large quantity of peat of excellent quality for fuel or fertilizer. The Orchid Humus Fertilizer Co., of Peekskill,

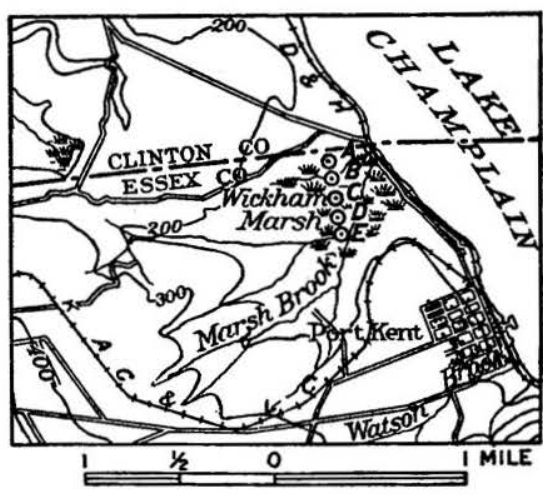

Figure 4.- Map of Wickham Marsh, Essex County, N. Y., showing approximate position of test borings for peat. and Asa McElhone, of Fishkill, are producing peat from this deposit for fertilizer or stock feed.

Locality 6.-A deposit about 1 mile west of Hopewell Junction, worked by Norwin G. Cole, of Hopewell Junction, has produced peat for fuel and fertilizer for several years. The deposit is small but is reported to contain good peat to a depth of 10 feet.

Locality 7.- Stissing Pond, near Pine Plains, is surrounded by a peat deposit that is reported to be about 10 feet thick and to be underlain by marl. ${ }^{37}$ Peat is now forming in the pond. The living vegetation, according to Parsons, consists of cat-tails, rushes, moss, and grasses.

\section{ESSEX AND FRANKIIN COUNTIES.}

Locality 8.-Test holes made in a marsh in Essex County, along the tracks of the Delaware \& Hudson Co., south of the lower mouth of Ausable River, failed to show peat.

Locality 9.-Wickham Marsh, about 1 mile northwest of Port Kent, near the mouth of Marsh Brook, contains a deposit about 160 acres in area and 7 feet in average thickness, which should yield approximately 224,000 short tons of air-dried peat. (See fig. 4.) The living vegetation consists chiefly of tamarack, spruce, and alder trees, heath shrubs, ferns, moss, cotton grass, reeds, cat-tails, pitcher plants, sedges, and sphagnum. The peat, which is composed chiefly of the remains of mosses, sedges, and grasses, is brown and fibrous

8 Parsons, A. L., Peat, its formation, occurrence, and uses in New York: New York State Mus. Fifty-seventh Ann. Rept., pt. 1, p. 72, 1903. 
in the upper layers but well decomposed at the bottom. Test borings gave the following results:

Logs of test borings in Wickham Marsh, 1 mile northoest of Part Kent, N. Y.

Hole A, about 100 yards south of north end of marsh near extremity of a north-south line through center:

Brown fibrous peat, containing roots of living plants---- $1 \frac{1}{2}$

Brown fibrous peat, composed chiefly of moss, sedges, and grasses

Feet.

Brown soft plastic peat of uniform texture

le $B, 100$ yards south of hole A :

Brown fibrous peat

Dark-brown well-decomposed plastic peat-_-_-_-_-_-_-_- 5

Hole C, 100 yards south of hole B :

Light-brown fibrous peat. 3

Dark-brown well-decomposed plastic peat_-_-_-_-_-_--- 7

Hole D, 100 yards south of hole C: Light-brown fibrous peat_- 3

Hole E, 100 yards south of hole D, near south margin of de-

posit: Brown fibrous peat.

2

Analysis 20 (p. 45) shows the character of a composite sample obtained by mixing peat taken at intervals of 2 feet in depth from each of the five test holes. It shows the peat to be low in ash and high in fixed carbon and nitrogen and therefore to be of good quality for fuel or fertilizer. Analysis 20a (p. 45) shows the composition of a specimen obtained by mixing peat taken from the upper 6 feet in each of the five test holes. The analysis shows only 6.87 per cent of ash. Better fuel could be made from this layer than from the lower layers, unless the peat is too fibrous. This deposit is well situated with respect to transportation facilities and contains some of the best peat in New York.

Locality 10.-A sphagnum-heath bog extending along Twobridge Brook

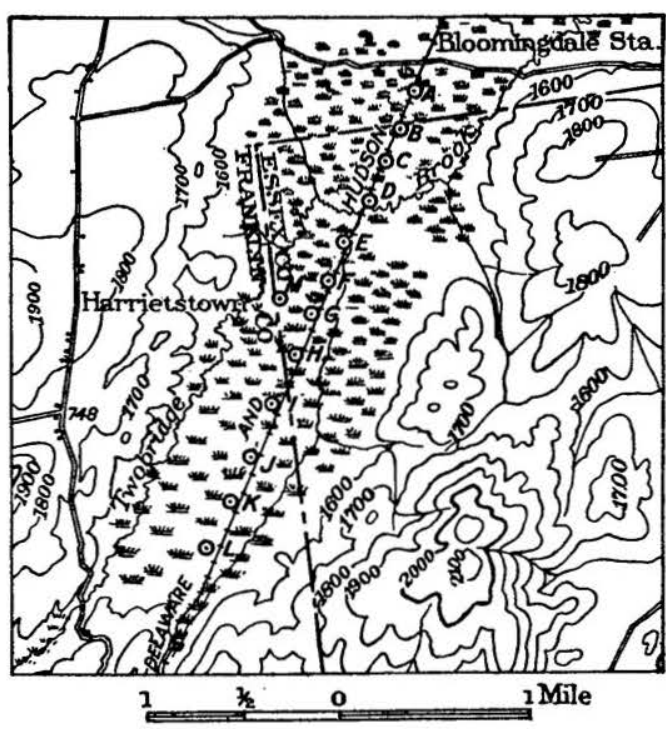

FIGURF 5.-Map of peat deposit southwest of Bloomingdale station, Essex and Franklin counties, N. Y., showing approximate position of test borings for peat.

for about 3 miles southwestward from Bloomingdale station covers 1,500 acres, but not all of it is underlain by peat. (See fig. 5.) The tracks of the Delaware \& Hudson Co. pass through the center of 
the bog. The deposit of peat is about 700 acres in area and 5 feet in thickness and should yield approximately 700,000 short tons of air-dried peat. The living vegetation consists largely of heath shrubs, mosses, the blueberry, and cotton grass, though there are also a few scattered tamarack, spruce, and cedar trees. The deposit is of the built-up type and was formed on a flat, sandy, poorly drained surface. A series of test borings gave the following results:

Logs of test holes in bog 3 miles southwest of Bloomingdale station, Essex and Franklin counties, N. Y.

Hole A, about a quarter of a mile southwest of Bloomingdale station on east side of rallroad track: Light-brown mossy fibrous peat

Hole B, about a quarter of a mile southwest of hole A : Same character of material as that in hole $A$.

Hole C, about a quarter of a mile southwest of hole B : Same character of material as that in hole $\mathbf{A}$.

Hole D, about a quarter of a mile southwest of hole C: Same character of material as that in hole $\mathrm{A}$.

Hole E, about a quarter of a mile southwest of hole $\mathrm{D}$, near bridge across Twobridge Creek: Light-brown mossy fibrous peat

Hole F, about a quarter of a mile southwest of hole $\mathrm{E}$ :

Light-brown mossy fibrous peat

Dark-brown decomposed plastic peat

Hole G, about a quarter of a mile southwest of hole F :

Light brown mossy fibrous peat

Dark-brown well-decomposed plastic peat

Hole $\mathbf{H}$, about a quarter of a mile southwest of hole $\mathbf{G}$ :

Light-brown mossy fibrous peat

Dark-brown well-decomposed plastic peat

Hole I, about a quarter of a mile southwest of hole $\mathbf{H}$ :

Light-brown mossy fibrous peat

Dark-brown well-decomposed plastic peat

Hole J, about a quarter of a mile southwest of hole I :

Light-brown mossy fibrous peat

Dark-brown well-decomposed plastic peat of uniform texture

Hole $\mathbf{K}$, about a quarter of a mile southwest of hole $\mathbf{J}$ :

Light-brown mossy fibrous peat

Brown well decomposed plastic peat of uniform texture

Hole $\mathrm{L}$, about a quarter of a mile southwest of hole $\mathrm{K}$, near south edge of bog: Light-brown mossy fibrous peat.

Hole M, about a quarter of a mile west of hole F : Dark-brown fibrous mossy peat.

In hole $\mathrm{K}$ a layer of logs, roots, and woody matter was encountered at a depth of 12 feet that could not be penetrated with the sampling tool.

Analysis 21 (p. 45) shows the character of a composite sample obtained by mixing peat taken at intervals of 2 feet in depth from 
holes $\mathrm{E}, \mathrm{F}, \mathrm{G}$, and $\mathrm{M}$. It shows that the peat in the northern half of the bog contains only 6.54 per cent of ash and is therefore well adapted for fuel. The heating value is 9,125 British thermal units. Analysis 22 (p. 45) shows the character of a composite sample obtained by mixing peat taken at intervals of 2 feet in depth from holes $H, I, J$, and $K$. It shows that the peat in the southern half of the bog is also of excellent quality for fuel. The peat contains a fair percentage of nitrogen and hence might be used in the manufacture of commercial fertilizers.

Locality 11.-Two heath bogs on opposite sides of the Delaware \& Hudson Co.'s track, at Middle Kilns. The largest bog contains about 200 acres and lies south of the railroad station. The average thickness of the peat in this bog is reported to be about 6 feet. It is well cleared and could be readily drained. If the area and depth are correctly estimated it should yield approximately 240,000 short tons of air-dried peat.

Locality 12. - Sphagnum-heath bog, on the Chateaugay branch of the Delaware \& Hudson Co.'s railroad, a quarter of a mile southeast of Onchiota. This bog covers about 200 acres, and the deposit of peat is reported to be 5 feet in average thickness. It should therefore yield approximately 200,000 short tons of air-dried peat.

Locality 13.--A sphagnumspruce bog south of Lake Clear, about 2 miles west of the rail-

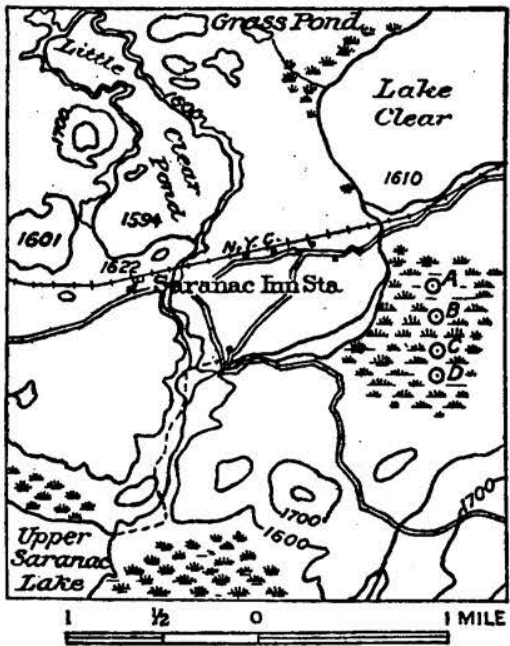

Figure 6.-Map of peat bog south of Lake Clear, Franklin County, N. Y., showing approximate position of test borings for peat (A, B, C, D).

road station at Lake Clear Junction, is about 200 acres in area, $2 \frac{1}{2}$ feet in average thickness, and contains about 100,000 short tons of air-dried peat. (See fig. 6.) The Adirondack division of the New York Central Railroad skirts the north end of this bog. The vegetation consists chiefly of sphagnum and other mosses, heath shrubs, and spruce and tamarack trees. The peat is dark brown and firm to fibrous and is composed chiefly of the remains of grasses and sedges, except near the top, where moss peat predominates.

A composite sample, whose character is shown in analysis 23 (p. 45), was obtained by mixing peat taken at intervals of 1 foot in depth from each of four test holes put down on a north-south line through the center of the bog. The peat appears to be low in ash and high in fixed carbon and nitrogen, which indicates that it is of good qual- 
ity for fuel or fertilizer. The deposit, however, is too shallow for the production of machine-peat fuel, though hand-cut peat might be obtained after the bog had been adequately drained.

Locality 14.-A series of test holes was made from northeast to southwest across certain areas south and east of the railroad station at Saranac Junction, on the Adirondack division of the New York Central Railroad, but no peat was found. The peat appears to have been destroyed by fire. The living vegetation is the same as that which grows on many peat bogs in the surrounding region and is therefore misleading.

Locality 15.-A bog containing peat extends from the southeast shore of Raquette Pond to the north shore of Tupper Lake and east-

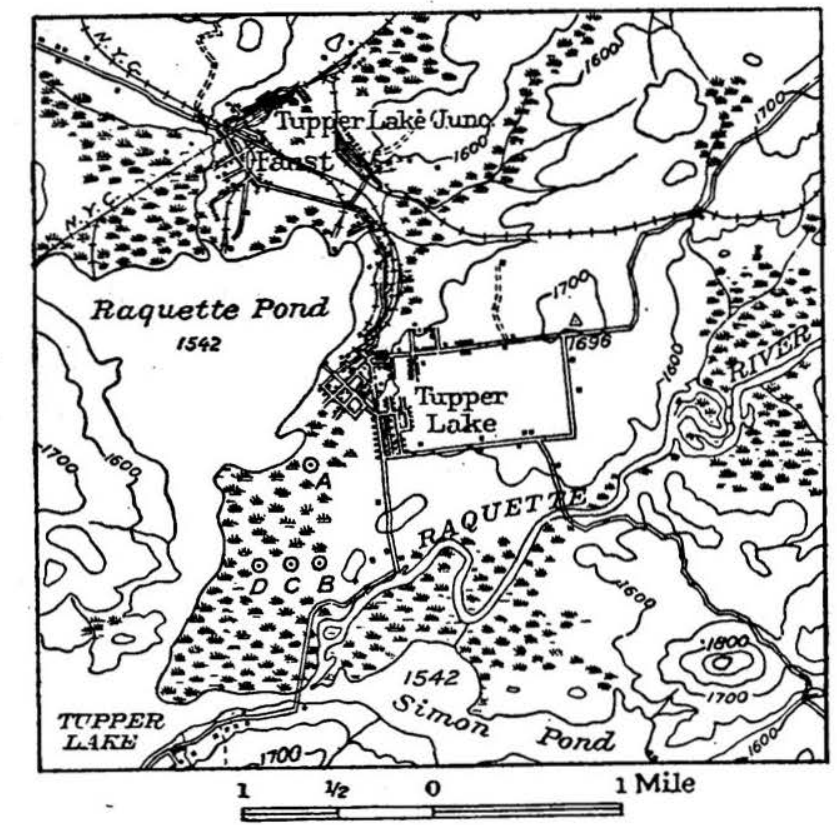

Frgdre 7.-Map of peat deposit northwest of Tupper Lake, Franklin County, N. Y., showing approximate position of test borings for peat.

ward along Raquette River. (See fig. 7.) The part of the bog adjacent to Raquette Pond and Tupper Lake is about 600 acres in area, and the peat is 5 feet in average thickness, so that the area would yield approximately 600,000 short tons of air-dried peat. This area was formerly a heavily wooded swamp, but the timber has been removed. The peat is dark brown and well decomposed; it contains some soft woody matter near the top and the remains of sedges, grasses, and cat-tails at the bottom. The surface is covered by a thin layer of mud, which has probably been deposited by recent floods. A series of test borings gave the following results: 
Thiokness of peat in test borings in deposit northwest of Tupper Lake, N. Y.

Hole A, near north end of deposit: ' Feet.

Black muck

$\frac{1}{4}$

Brown flbrous peat

$\frac{3}{4}$

Dark-brown plastic peat

4

Hole $B$, near east-central part: Dark-brown plastic peat_-.---- 4

Hole C, about 100 yards west of hole B: Same kind of peat as that in hole $B$

Hole D, about 100 yards west of hole C: Same kind of peat as

that in hole C.

Analysis 24 (p. 45), of a sample taken from hole A, shows the peat in the northern part of the bog to be of good quality for fuel or fertilizer. Analysis 25 (p. 45) shows the character of a composite sample obtained by mixing peat taken at intervals of 1 foot in depth from holes B, C, and D, and indicates that the peat in the central part of the bog is also of good quality.

\section{GENESEE AND ORLEANS COUNTIES.}

Locality 16.-The Oak Orchard Swamp, which extends from Clarendon, Orleans County, westward for a distance of nearly 20 miles,

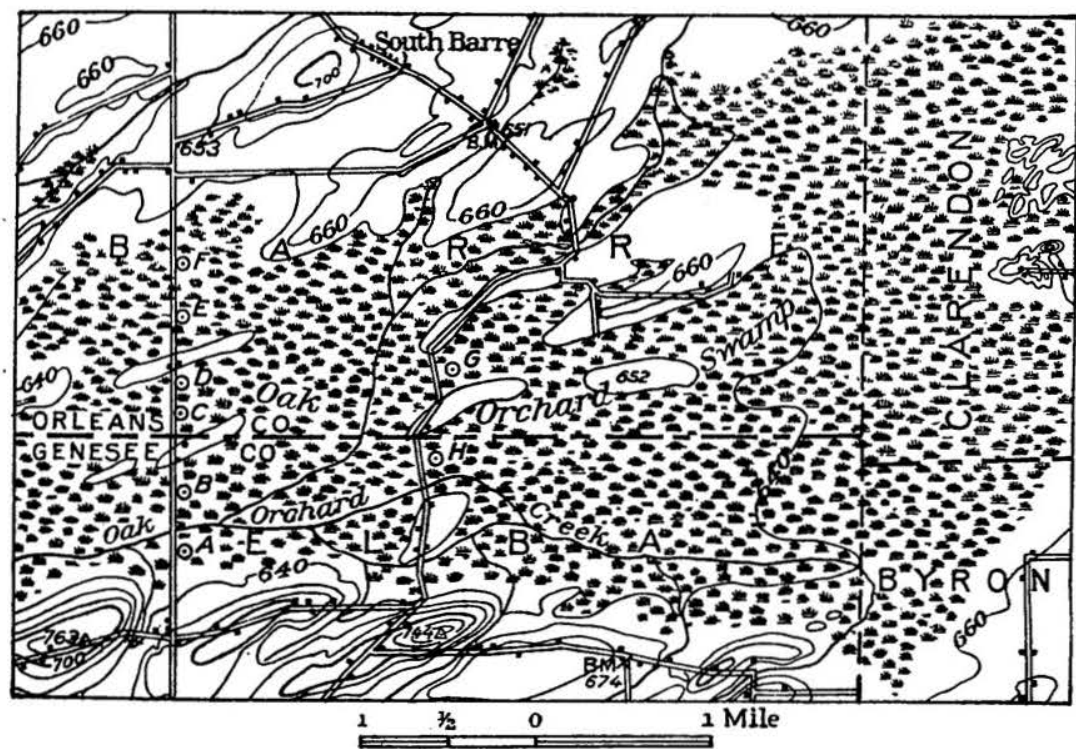

Brgurs 8.-Map of the eastern part of Oak Orchard Swamp, Genesee and Orleans counties, N. Y., showing approximate position of test borings for peat.

is one of the largest in New York. (See fig. 8.) Much of the peat land in locality 16, which includes the eastern part of the swamp, 9 miles north of Batavia, in Genesee and Orleans counties, is owned by the Western New York Farm Land Co., of Batavia, N. Y. The average width of the swamp is about 2 miles. Approximately 10 
square miles of the total area is covered with peat. The peat is shallow throughout most of the swamp, especially in the western part, where the average thickness is only 3 feet. In the eastern part the peat ranges in thickness from 1 to 10 feet. The swamp probably would yield about 1,500,000 short tons of air-dried peat from beds 6 feet thick and an equal quantity from beds 3 feet thick. The early flora consisted of a dense growth of small plants as well as maple and other deciduous trees and some white cedar. However, the area has been largely cleared of trees and undergrowth, and thousands of acres are used for the growth of celery, lettuce, onions, and buckwheat.

A series of test borings in this locality gave the following results:

Logs of test borings in Oak Orchard Swamp, 9 miles north of Batavia, N. Y.

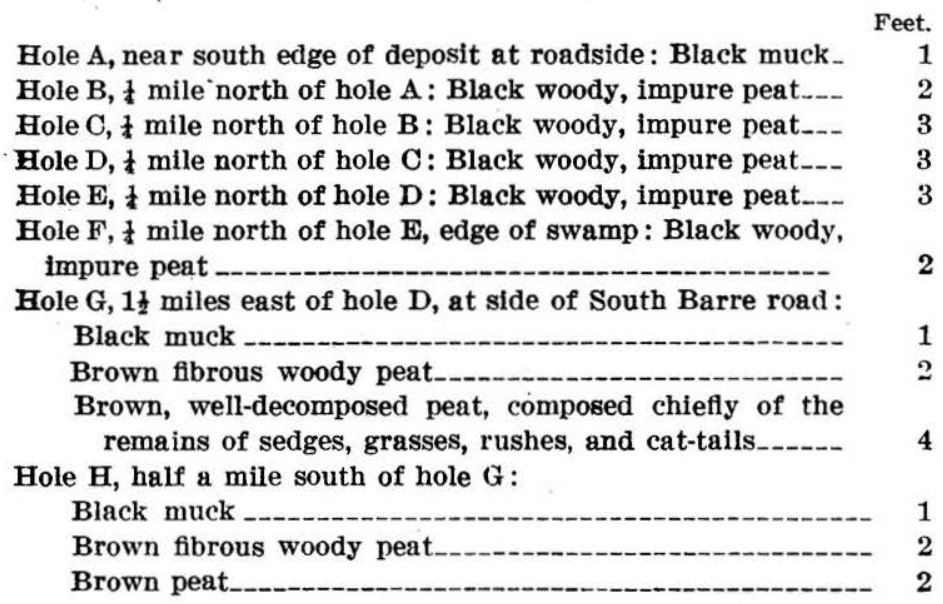

Analysis 69 (p. 45) shows the character of a composite sample obtained by taking peat at intervals of 1 foot in depth from holes B, $\mathrm{C}, \mathrm{D}$, and $\mathrm{E}$ and from several drainage ditches. It indicates that the peat is of good quality. The high nitrogen content (3.01 per cent) is especially noteworthy. Analysis 70 (p. 45) shows the character of a composite sample taken from hole $G$, where the deepest peat was found. It indicates that the peat in this part of the deposit is similar to that in holes A, B, C, D, E, and F. The peat throughout this locality is of excellent quality for fertilizer or fuel.

Locality 17.-Locality 17 includes the western part of the Oak Orchard Swamp, north of the villages of Alabama, Wheatville, and Oakfield, Alabama Township. The location and outline of this part of the swamp are shown on the map of the Medina quadrangle, published by the United States Geological Survey. As this locality has been described by Parsons, no borings were made. The average 
thickness of the peat, which is underlain by marl, is reported to be about 3 feet. Parsons ${ }^{88}$ says:

North of Alabama the deposit can hardly be dignified with the name of peat, for It is drier than in the other portions and has a sandy subsoll and the peat contains a large percentage of foreign matter. The forest growth is principally made up of white cedar, though many deciduous trees are found. Sphagnum is not common but has been replaced by a moss of the genus Hypmum.

. It therefore seems improbable that the western part of the Oak Orchard Swamp contains peat valuable for fuel.

\section{HERKIMER OOUNTY.}

Locality 18.-A spruce bog north of Brandreth extends along both sides of Beaver River and several small tributaries near the west end of Nehasane Lake. The location and area of the deposit are shown on the map of the Big Moose quadrangle, published by the United States Geological Survey. The living vegetation consists chiefly of black spruce trees, sphagnum, and heath shrubs. The thickness of the peat was not determined and no samples were taken for analysis.

Locality 19.-A meadow along the north branch of Moose River, about 2 miles north of Old Forge, is shown on the map of the Old Forge quadrangle published by the United States Geological Survey. This area contains no peat of economic value. The surface layer consists of muck, which contains much silt washed in by the overflow of Moose River. No samples were taken for analysis.

Locality 20.-A meadow on the north side of the New York Central Railroad track, about 1 mile southwest of Fulton Chain, is shown on the map of the McKeever quadrangle published by the United States Geological Survey. The peat in this meadow is too sandy to be of economic value and no samples were taken for analysis.

ONONDAGA COUNTY.

Locality 24.-Cicero Swamp, which lies about 3 miles east of North Syracuse and an equal distance south of Oneida Lake, is several miles long, though the east end contains some patches of dry land. (See fig. 9.) The whole area is shown on the maps of the Syracuse and Chittenango quadrangles published by the United States Geological Survey. The area shown in figure 9 is about 8 square miles, or 5,120 acres. The maximum thickness of the peat shown in test holes was 11 feet, but it is reported that the peat is 15 feet deep in some places. An area of about 3,500 acres is covered with peat 6 feet deep and 1,500 acres with peat 4 feet deep. The quantity of peat available in locality 24 is estimated to be equivalent to $5,400,000$ short tons of air-dried peat. The area is composite in origin. Most of the peat is of the built-up type, though pond-peat is found in some places, indi-

${ }^{28}$ Parsons, A. L., op. cit., pp. 83-85.

$91065^{\circ}-22-10$ 
cating that the bog was formed on a large flat, poorly drained surface that contained small basins. Marl underlies the peat in some places. The living vegetation consists chiefly of spruce, tamarack, and cedar trees, heath shrubs, and sphagnum. Near the center of the swamp there is a large open sphagnum heath similar to those in Maine, Minnesota, and eastern Canada. Around the margin of the swamp stretches a zone one-fourth of a mile wide of deciduous trees, chiefly red maples. Plate $\mathrm{XI}, A$, shows the general appearance of the swamp. The peat, which is composed chiefly of the remains of

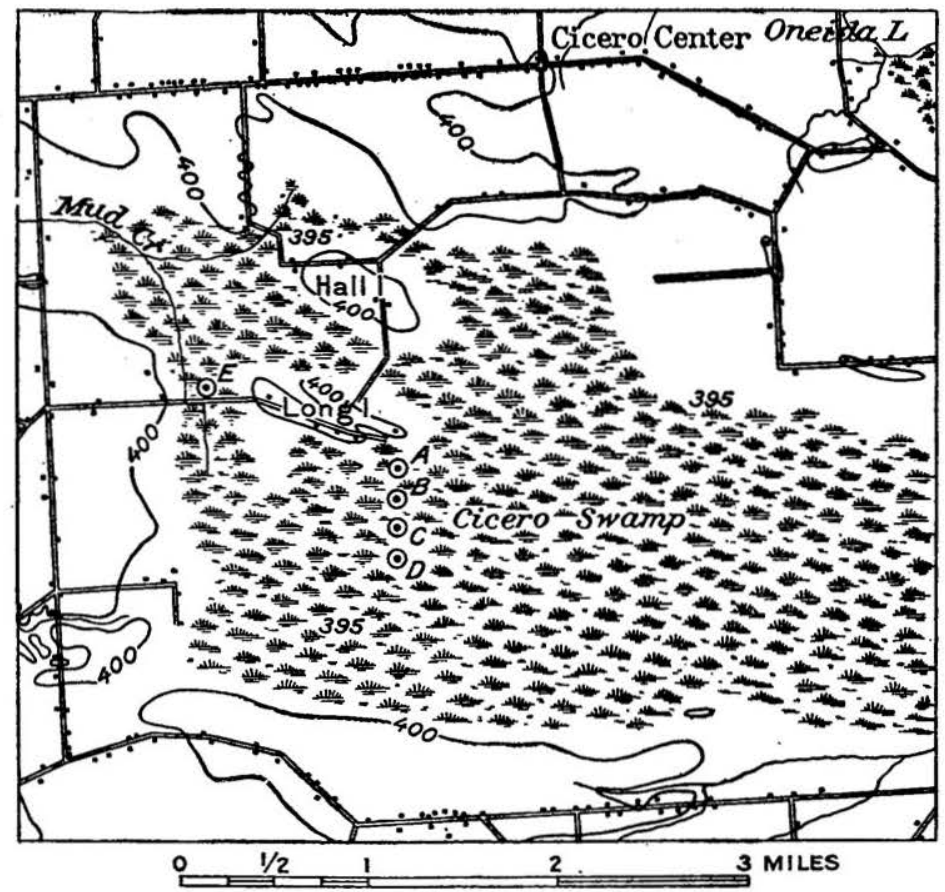

Figure 9.-Map of part of Cicero Swamp, Onondaga County, N. Y., showing approximate position of test borings for peat.

sphagnum moss, heath shrubs, and grasses, is brown, fibrous to welldecomposed, soft, and plastic. The upper layers contain some woody matter. A series of test boring gave the following results:

Logs of test borings in Cicero Swamp, near North Syracuse, $N$. $\boldsymbol{Y}$.

Hole A, south of east end of Long Island: Black woody impure peat._._._._. 2

Hole B, one-eighth of a mile south of hole A :

Black woody peat and muck. 2

Brown plastic peat__________ 2

Hole C, one-eighth of a mile south of hole B :

Brown fibrous peat containing woody matter._._._.- 2

Dark-brown well-decomposed peat 


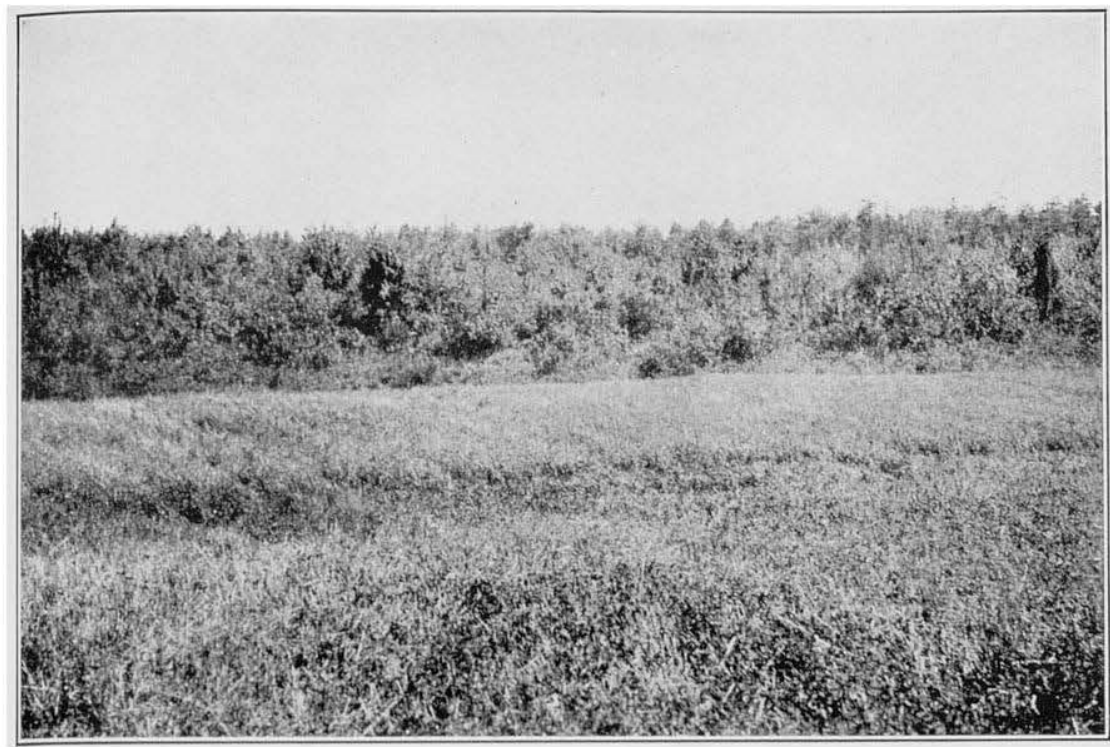

A. NATIVE SECTION OF CICERO SWAMP, ONONDAGA COUNTY, N. Y.

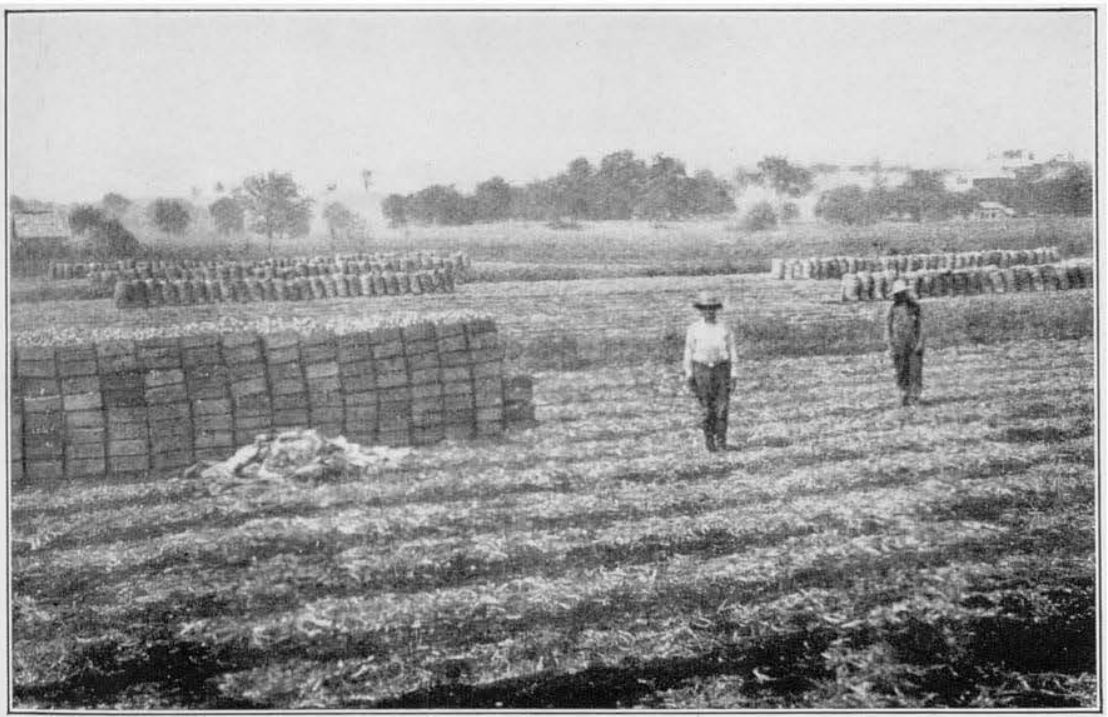

B. RECLAIMED PEAT SWAMP IN THE WALLKILL RIVER VALLEY NEAR BIG ISLAND, ORANGE COUNTY, N. Y.

Used for the cultivation of onions.

The Winning of a Peat Swamp. 

Hole D, one-eighth of a mile south of hole C:

Feet.

Brown flbrous peat, containing the remains of sphagnum

Dark-brown well-decomposed plastic peat of uniform texture

Hole E, midway between the west end of Long Island and the west edge of the swamp:

Brown fibrous, woody peat

Brown well-decomposed plastic peat of uniform texture

Analysis 64 (p. 45) shows the composition of a composite sample obtained from hole $\mathrm{D}$ and indicates that the peat near the center of the swamp is of excellent quality for fuel or fertilizer. The ash content is only 7.34 per cent and the nitrogen 2.02. The calorific value is 9,256 British thermal units. Analysis 65 (p. 45), which shows the character of a composite sample taken from hole $\mathrm{E}$, indicates that the peat near the western edge of the deposit is similar to that in the center.

Cicero Swamp is well situated for the production of peat fuel or fertilizer, and the deposit contains a large quantity of good peat. Much of the deposit is underlain by marl, which could be economically used in neutralizing the peat. A large area of peat at the west end of the swamp is used for the growth of truck crops.

Locality 25.-A deposit about $1 \frac{1}{2}$ miles northwest of North Syracuse, Clay Township, which is about 1,500 acres in area and 10 feet in average thickness, should yield about $3,000,000$ short tons of airdried peat. The peat, which is made up chiefly of the remains of sedges, grasses, leaves, and shrubs, is dark brown, well decomposed, plastic, and of uniform texture. The early flora consisted chiefly of deciduous forest trees and partly of spruce and tamarack, but the trees have been cut, and a large part of the area has been drained and cleared and is cultivated. Onions, celery, and lettuce are the principal crops grown. A series of test borings gave the following results.

Logs of test borings in peat deposit $1 \frac{1}{2}$ miles northwest of North Syracuse, N. Y.

Hole A, about 50 yards north of sonth edge and three-fourths Page. of a mile from east end :

Brown fibrous peat

Brown well-decomposed firm peat____________-_ 4

Hole B, about 150 yards north of hole A :

Brown fibrous peat, dry and earthy.

Brown well-decomposed dry peat.

Brown well-decomposed plastic peat of uniform texture --- $\quad 8 \frac{1}{2}$

Hole C, about 100 yards north of hole $B$ in center :

Brown dry fibrous earthy peat.

1

Brown well-disintegrated dry peat.

Brown well-decomiposed plastic peat 12

Analysis 66 (p. 45) shows the composition of a composite sample obtained from hole $B$ and indicates that the peat is well adapted for fuel or fertilizer. 
ORANGE COUNTY.

Locality 26.-A small deposit about 2 miles from Monroe on the Woodhuld farm occupies a filled basin that is underlain by marl. The living vegetation consists of cat-tails, grasses, and sedges. The deposit is less than 1 acre in area, is 4 feet in average thickness, and contains only about 800 short tons of air-dried peat. A thin layer of black muck covers the surface of the deposit. The upper layers of peat are fibrous, and the lower layers, which are underlain by

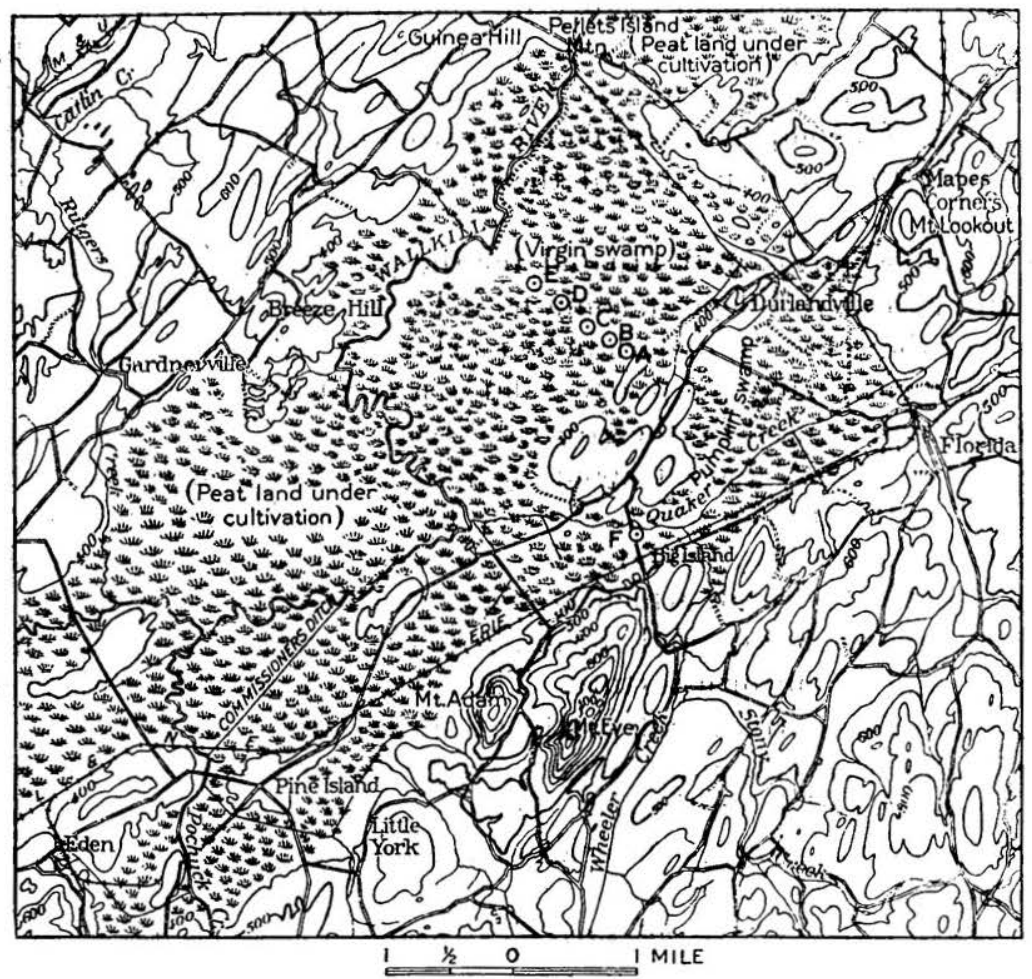

FiguRw 10.-Map of the " drowned lands" of Wallkill River valley, Orange County, N. Y., showing approximate position of test borings for peat.

marl, are well decomposed. Analysis 71 (p. 45) shows the composition of a specimen from this deposit and indicates that the peat is of good quality for fertilizer. This peat area, though small, is described because the owners, Woodhuld \& Lamereaux, have produced peat fertilizer from it for many years. There was no production in 1918 because of the small quantity of peat in the deposit, and it is believed that no further production will be attempted.

Locality 27.-The "drowned lands" of the Wallkill River valley extend through Warwick, Wawayanda, and Goshen townships. (See fig. 10.) Florida, Big Island, Pine Island, and Durlandville 
are situated along the southern margin of this swamp, which is about 17,000 acres in area and is underlain by peat that ranges in thickness from 1 to 25 feet. An area of about 10,000 acres is underlain by peat 10 feet deep. This deposit should yield approximately $20,000,000$ short tons of air-dried peat. The greater part of the area has been cleared and drained and is cultivated. The earlier vegetation, a living remnant of which may still be seen about 2 miles northwest of Big Island, consisted of spruce, tamarack, cedar, and red maple trees, shrubs, cat-tails, rushes, sedges, grasses, and mosses. The peat is dark brown and fine grained except near the bottom, where green pond peat occurs. Little fibrous peat was found, and no roots or buried logs are present except near the surface. A series of test borings gave the following results:

Logs of test brings in the "drowned lands" of Wallkill River valley, N. Y.

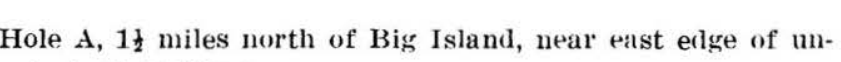
drained section:

Brown disintegraterl peat, containing some woody matter

Feet.

Dark-brown well-decomposed plastic peat of uniform texture, underluin by chay

Hole $\mathrm{B}$, about 100 yards northwest of hole $\mathrm{A}$ :

Brown woody peat

Dark-brown well-decomposed plastic peat, uniform in texture

Greenish fine-grained pond peat

Hole C, about 100 yards northwest of hole B: Same kind of peat as that in hole $B$.

Hole D, about 100 yards northwest of hole C: Same kind of peat as that in hole $B$.

Hole E, about 100 yards northwest of hole D: same kind of peat as that in hole $B$.

Hole F, about a quarter of a mile northwest of Big Island, in area known as the "Pumpkin Swamp":

Brown ary well-disintegrated earthy peat

Dark-brown thoroughly decomposed plastic peat, of uni-

form texture

Greenish fine-grained clayey pond peat_........... 2

Parsons ${ }^{39}$ gives the following results of test borings for peat at other places in this locality:

Depth of peat in test borings in "drouned lands" of Wallkill River valley, N. Y.

Boring on Black Walnut Island

Feet.

Boring half a mile west of Durlandrille

$18+$

Boring $1 \frac{1}{2}$ miles west of Durlandville

Boring half a nile west of Big Island 17

Boring 1 mile west of Big Island

Boring at Florida 
Analysis 72 ( p. 45) shows the character of a composite sample ob. tained by mixing material taken at intervals of 1 foot in depth from hole B. It shows that the peat is low in ash (13.68 per cent) and high in nitrogen ( 2.89 per cent) and therefore is suitable for fuel and fertilizer. Analysis 73 (p. 45) shows the character of a composite sample from hole $F$. It indicates that the peat in this part of the deposit contains a higher proportion of inorganic matter than that in the vicinity of hole B. The nitrogen content of both samples is relatively high. This deposit, which contains peat of excellent quality for both fuel and fertilizer, is the largest in New York. The area is extensively used for the cultivation of onions and other crops and is probably more valuable for this use than for the production of peat for fuel. (See Pl. XI, B.) The onions are grown on relatively pure peat. About 2,000 acres of the Wallkill Swamp are undrained.

ST. IAWRENOE COUNTY.

Locality 28.-A bog west of Horseshoe Lake, about half a mile south of Horseshoe station, Piercefield Township, is shown on the map of the Tupper Lake quadrangle, published by the United States Geo. logical Survey. This bog is about 250 acres in area. The peat is shallow and therefore of doubtful value.

Locality 29.-A bog on the east shore of Hitchins Pond, Colton Township, is about 200 acres in area and 4 to 5 feet in average thickness. This deposit should yield approximately 180,000 short tons of air-dried peat. The surface is smooth and is overgrown by sphagnum moss and heath shrubs.

\section{SENECA AND WAYME COUNTIES.}

Montezuma Marsh, north of Cayuga Lake, is divided at the north end into two arms, one extending along Seneca River and the other along Clyde River. Below the junction of these rivers the marsh is broad and extends to Cayuga Lake. Its area and location are shown on the maps of the Auburn, Clyde, Geneva, and Weedsport quadrangles published by the United States Geological Survey. Its maximum length from Cayuga Lake northward to Spring Lake is about 14 miles. The total area of the Montezuma Marsh is approximately 24 square miles; the two northern branches are each about 7 square miles in area, and the widest part, at the north end of Cayuga Lake, near the junction of Clyde and Seneca rivers, is about 10 square miles in area. Only a part of the marsh contains peat and muck, much of which is shallow. In the northern part of the eastern branch the average thickness of the deposit is only 3 feet. Farther south toward the center and also in the western branch along Clyde River the deposit is deeper. The part adjoining the north end of Cayuga Lake contains filled-basin peat and muck underlain by marl, a condition which indicates that the lake formerly extended farther north 
than its present shore line. The living vegetation on the margin of the marsh consists of small willow, maple, and alder trees, which grow in a zone oneeighth to one-half mile wide. In the area surrounded by this zone the dominant plants are cattails, sedges, and marsh grasses, which are gathered and sold for packing material and chair seats. The peat is fibrous to plastic and rather impure. The greater part of the material in this deposit is muck. A strong odor of hydrogen sulphide was noticeable when the holes were put down. Because of the large area of Montezuma Marsh the character of the peat in its two northern branches, from both of which samples were obtained, is described separately.

Locality 30. - Locality 30 (see fig. 11) includes the northeast branch of the marsh extending along Seneca River. It is estimated that the deposits in this locality, much of which is underlain by

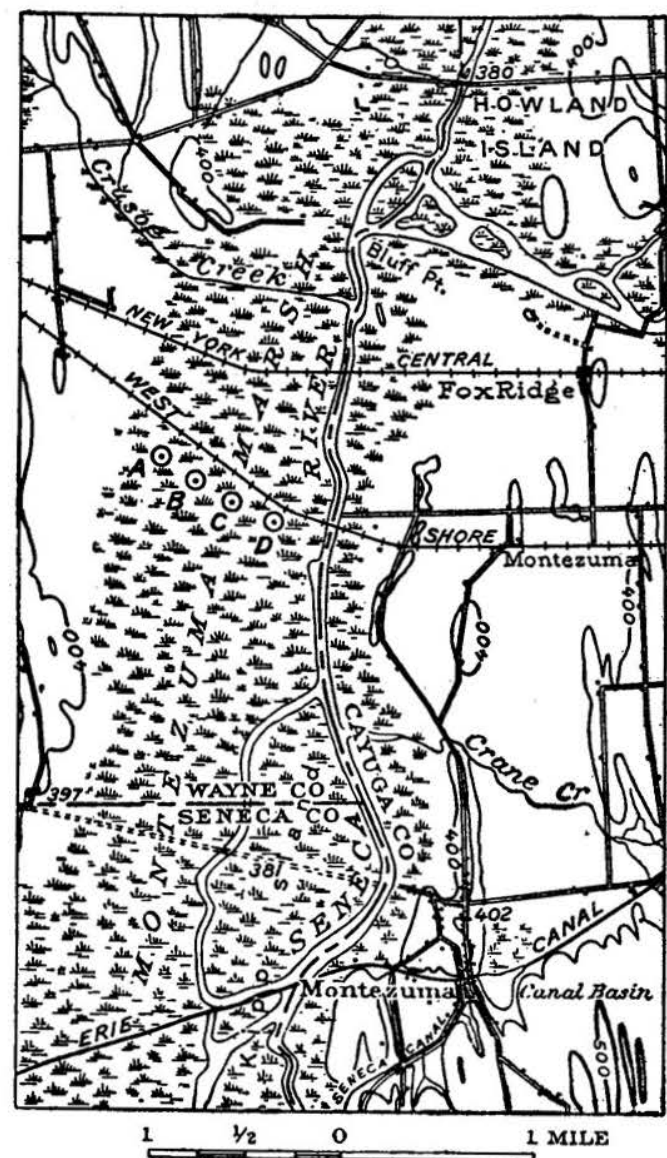

FigUra 11.-Map of the northeastern part of Montezuma Marsh, Seneca and Wayne counties, N. Y., showing approximate position of test borings for peat.

marl, should yield 2,500,000 short tons of air-dried peat. A series of test borings gave the following results:

Logs of test borings in northeastern part of Montezuma Marsh, on Seneca River, N. Y.

Feet.

Hole A, about 1 mile west of Seneca River, on the south side

of the tracks of the West Shore Railroad: Black muck _-__- 1

Hole B, about one-fourth of a mile east of hole A :

Black muck

1

Hole C, about one-fourth of a mile east of hole B :

Black muck.

Black plastic peat

Hole D, about one-fourth of a mile east of hole C: Black sandy muck 
Analysis 67. (p. 45) shows the character of a composite sample obtained by mixing material from holes $B$ and C. It indicates that this deposit contains a high percentage of ash and could not be used for fuel. The following analysis represents the composition of the marl that unplerlies the peat in this locality:

Analysis of marl from Seneca and Wayne counties, $N . Y$.

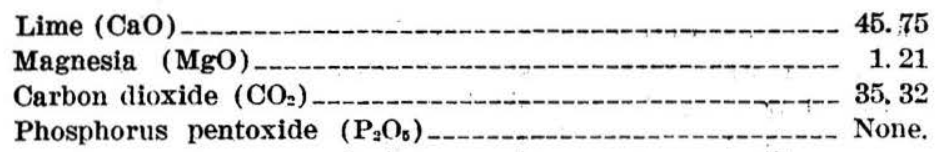

Although the peat in this part of the deposit is too shallow and too impure to be of any value for fuel, it might be used as an ingredient of fertilizers. However, the marl that underlies the deposit is of good quality and could be advantageously used for treating the peat or for the manufacture of Portland cement. Parsons ${ }^{40}$ states that the peat is also shallow at the south end of the marsh near Cayuga Lake.

Locality 31.-Locality 31 (see fig. 12) includes the northwest branch of Montezuma Marsh, along Clyde River. This branch is about 7 square miles in area and contains a deposit of peat 4 feet in average thickness, which should yield about $3,500,000$ short tons of air-dried peat. The peat in the center of this locality is underlain by marl. The vegetation consists chiefly of cat-tails and maple, willow, and alder trees. The peat is dark brown, firm, and somewhat fibrous near the surface but plastic and well decomposed in the lower half of the deposit. About 6 inches of black sandy muck overlies the peat. A strong odor of hydrogen sulphide was noticeable when the test borings were made. A series of holes gave the following results:

Logs of test borings in northwestern part of Montezuma Marsh, Seneca and

\section{Feet.}

Hole A, about a quarter of a mile west of the east edge of the marsh along the New York Central Railroad: No peat found.

Hole B, about a quarter of a mile west of hole A :

Black sandy peat.

Black fibrous peat_-

Hole C, about a quarter of a mile west of hole B :

Black sandy muck:

Brown fibrous peat

Brown plastic peat

\section{Wayme counties, N. Y.}

$\mathrm{D}$, about a quarter of a mile west of hole $\mathrm{C}$ :

Black sandy muck

Brown fibrous to disintegrated peat. 
Analysis 68 (p. 45), which represents the character of a composite sample from holes $\mathrm{C}$ and $\mathrm{D}$, shows that the material may be suitable for agricultural use, but is too high in ash for fuel. Locality 31 contains better peat and muck for agricultural use than locality 30 and is well situated for development, though drainage might be

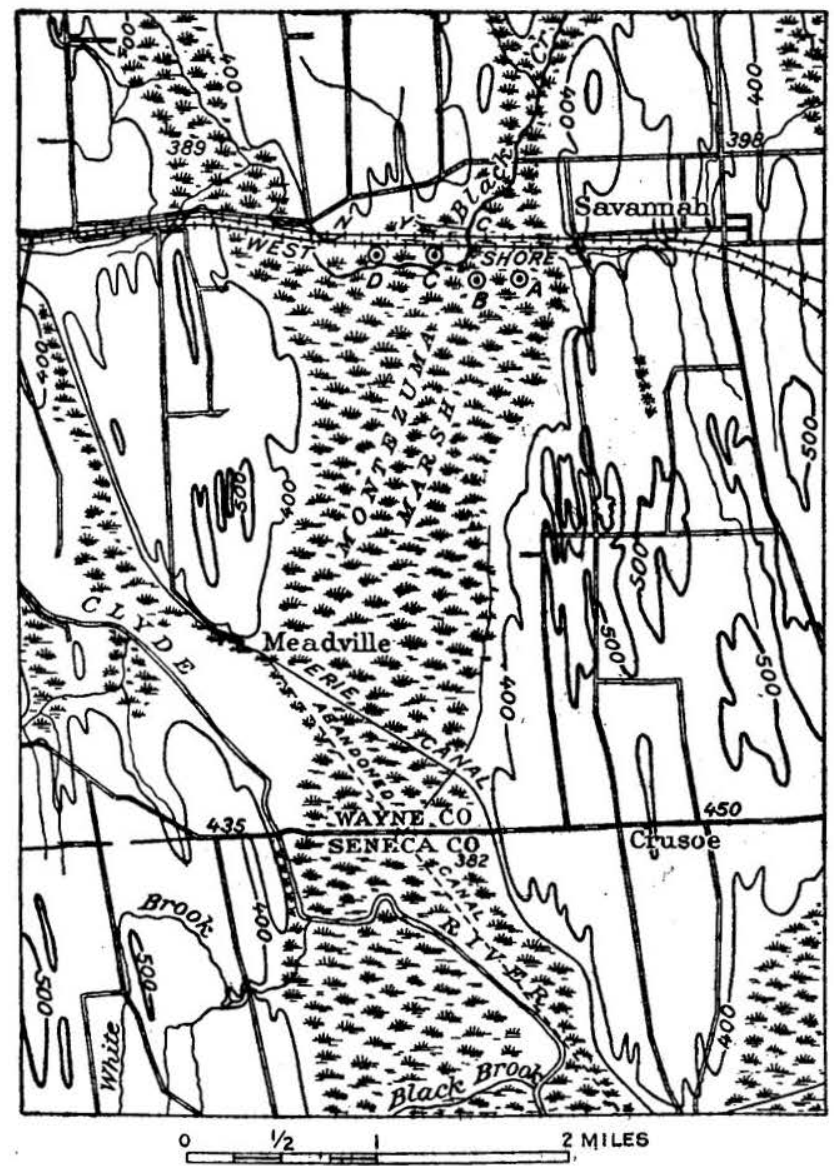

Figure 12.-Map of the northwestern part of Montezuma Marsh, Seneca and Wayne counties, N. Y., showing approximate position of test borings for peat.

difficult during the high-water stages of Cayuga Lake and Clyde River. Several large drainage ditches already have been constructed. The marl underlying much of the peat is valuable for agricultural use or for the manufacture of Portland cement.

NEW JERSEY.

GENERAL FEATURES.

As shown on the accompanying map (Pl. XII), Bergen, Essex, Hudson, Mercer, Middlesex, Morris, Passaic, Somerset, Sussex, Union, and Warren counties, in the northern half of New Jersey, 
contain most of the peat in that State. The best and most exten. sive deposits occur in the Sprout Brook Meadow, Bergen County; in the Black, Bog and Vly, Troy, Vernon, and Sussex meadows, Morris County; and in the Pequest Meadows, Warren County. Some peat is also found along the coast. The deposits of northern New Jersey are chiefly of the meadow type, although there are a few spruce and cedar swamps in the State. The greater part of the peat originated in glacial lakes and ponds and should, therefore, be classed with the peat in the region of the Great Lakes and the New England States. Although most of the deposits in salt marshes along the

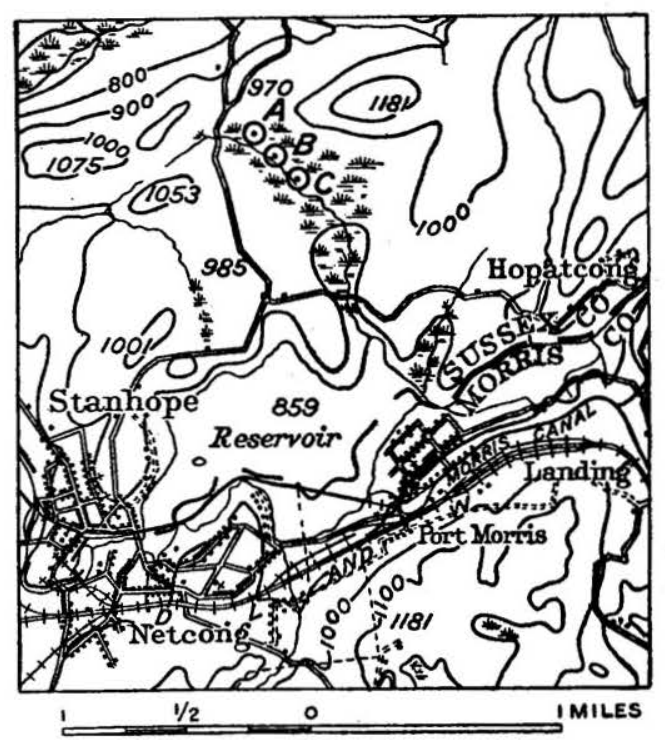

Fradre 13.-Map of peat deposit in Byram Township, Sussex County, N. J., showing approximate position of test borings for peat. coast of New Jersey, like many of those along the coast of New England, are related in origin and composition to those in the States of the southern Atlantic coastal region, these salt marshes are in this report classed with the major areas of peat accumulation of the northern region. It is estimated that about $15,000,000$ short tons of air-dried peat could be obtained from the deposits in New Jersey.

A detailed description of many of the valuable peat deposits in New Jersey is given in a report by Parmelee and McCourt. ${ }^{41}$

The following deposits from which peat has been produced commercially were examined in order to obtain data for this report.

SUSBEX COUNTY.

Locality 1.-A deposit in Byram Township, about $2 \frac{1}{2}$ miles north of Netcong, which is about 150 acres in area and 10 feet in average thickness, should yield approximately 300,000 short tons of air-dried peat. (See fig. 13.) Formerly the area was covered by a heavy growth of broad-leaved trees, among which the maple predominated. A large part of the surface has been cleared, and in other places there is a growth of cat-tails, sedges, grasses, and small shrubs. The de-

4 Parmelee, C. W., and McCourt, W. E., A report on the peat deposits of northern New Jersey : New Jersey Geol. Survey Ann. Rept. for 1905, pp. 223-313, 1906. 


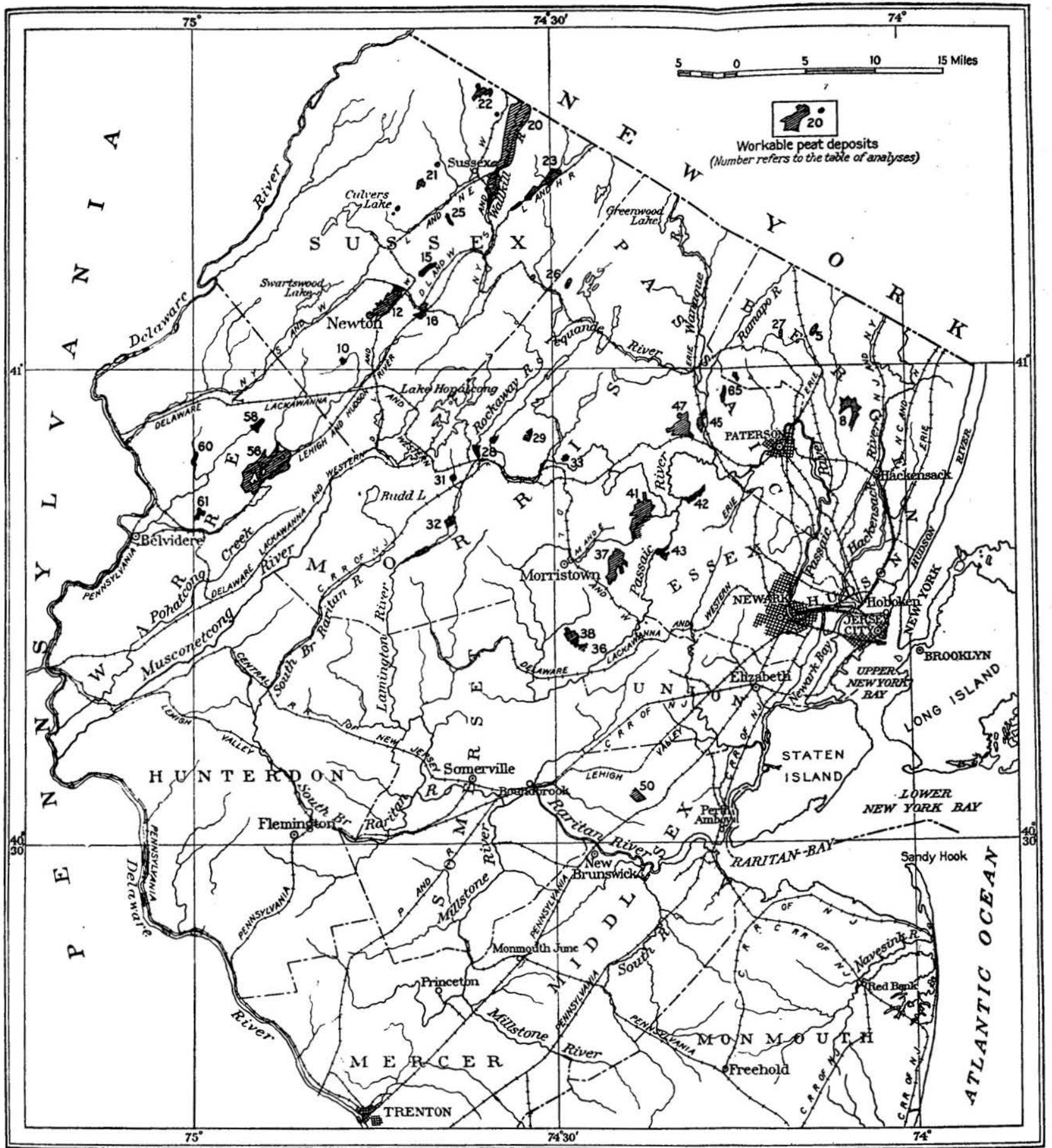

MAP SHOWING DISTRIBUTION OF PEAT IN NORTHERN NEW JERSEY. 

posit originated in a lake or pond, but the upper layers consist of built-up peat formed by the accumulation of the remains of plants that gained a foothold upon the peat after the basin had been filled. The origin is plainly shown in the structure of the deposit. The lower portion consists of greenish pond peat that is composed en. tirely of the remains of aquatic plants, such as pondweed and water lilies. Above this part, extending from the surface to a depth of about 10 feet, the peat is brown and is composed of the remains of grasses, sedges, mosses, cat-tails, shrubs, and other plants that grow only near the ground-water level. Several test holes put down in the northern half of the deposit gave the following results:

Logs of test borings in Byram Township, Sussex County, N. J.

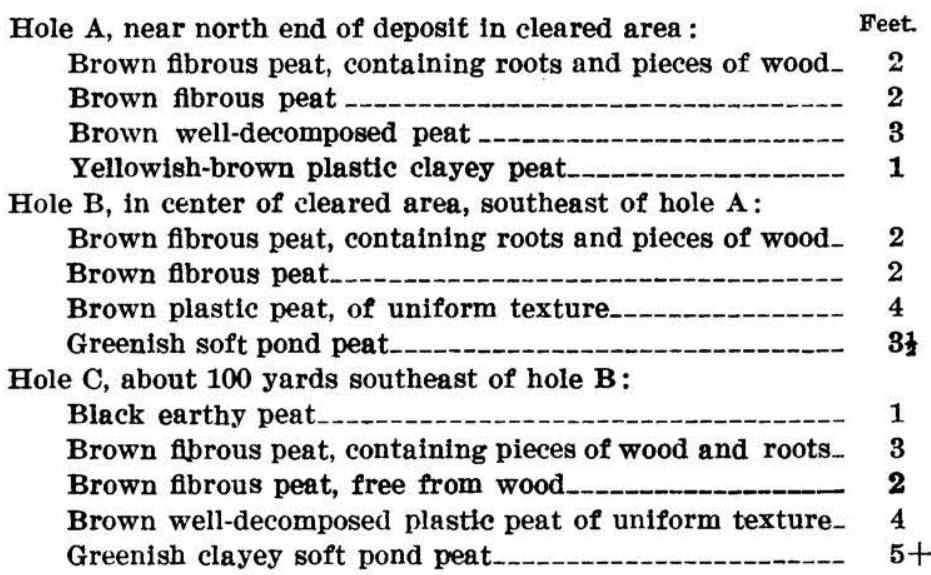

A composite sample (analysis 1, p. 44) from the upper 10 feet was obtained by mixing peat taken at intervals of 2 feet in depth from each of the test holes. The analyses show that the peat in the upper 10 feet is of good quality for fertilizer or for fuel. Peat fertilizer is being produced from this deposit by the Commercial Humus Co., of Newark, N. J. The peat is excavated by hand, loaded into wooden tram cars, and hauled by cable to the loading station, where it is macerated and discharged into a large rotary drier. From the drier the disintegrated peat is loaded directly into railroad cars. The product is used as a nitrogenous ingredient of commercial fertilizers. For several years a small quantity of peat fertilizer has been annually produced at this plant. Analysis 2 (p. 44) shows the quality of the raw peat excavated for fertilizer.

\section{WARREN COUNTY.}

Locality 2.-The plant of the Alphano Humus Co., of New York, N. Y., is located on the deposit in the Pequest Meadows, which ex- 
tend along Pequest River from Danville to Long Bridge. (See fig. 14.) About 2,000 acres, which it is estimated would yield approximately 1,600,000 short tons of air-dried peat, is said to be controlled by this company. The deposit is used for the growth of celery, onions, lettuce, and other truck crops, and for the production of peat fer-

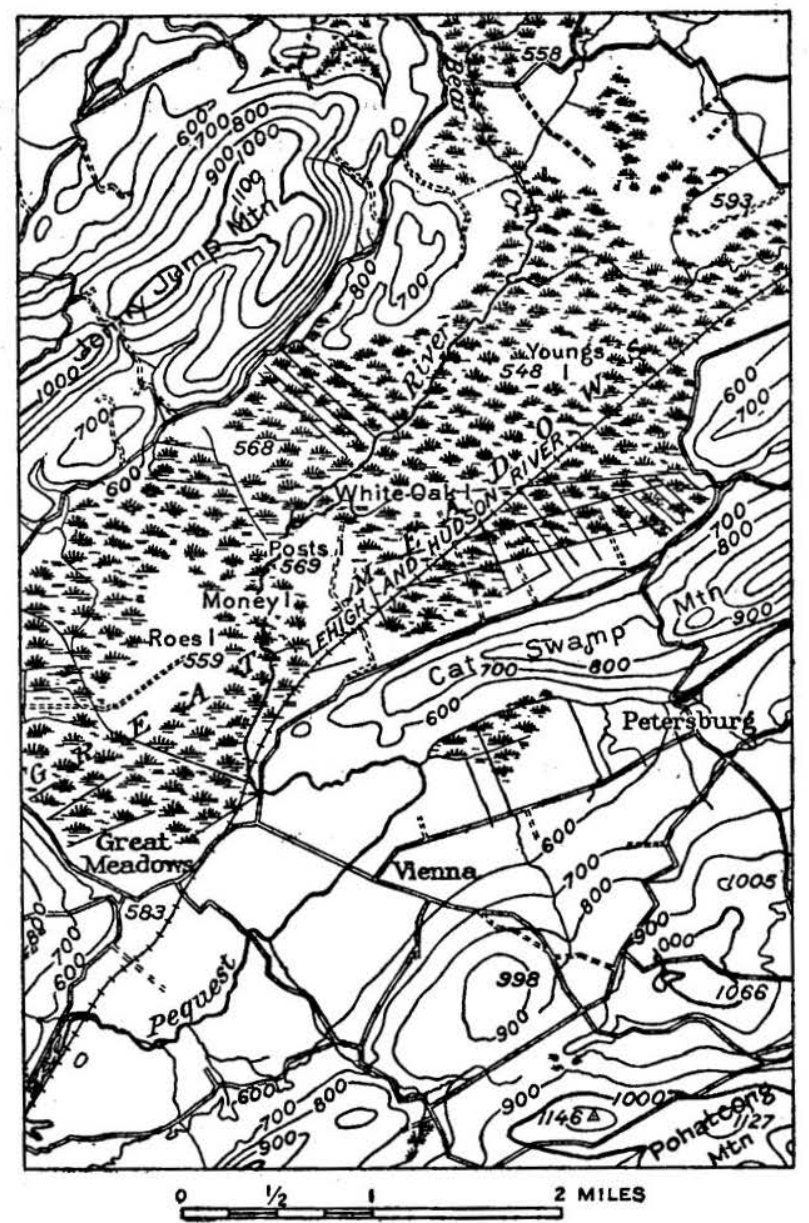

Figurn 14.-Map of peat deposit along Pequest River, Warren County, N. J.

tilizer. This locality contains some of the best peat in the United States and is well situated with respect to transportation facilities and market.

\section{MAINE.}

The most accessible peat deposits in Maine occur in Androscoggin, Cumberland, Kennebec, Knox, Lincoln, Oxford, Penobscot, Waldo, Washington, and York counties. (See Pl. XIII.) All these counties lie in the eastern and southern parts of the State and are traversed by railroads. Aroostook, Piscataquis, and Somerset coun- 


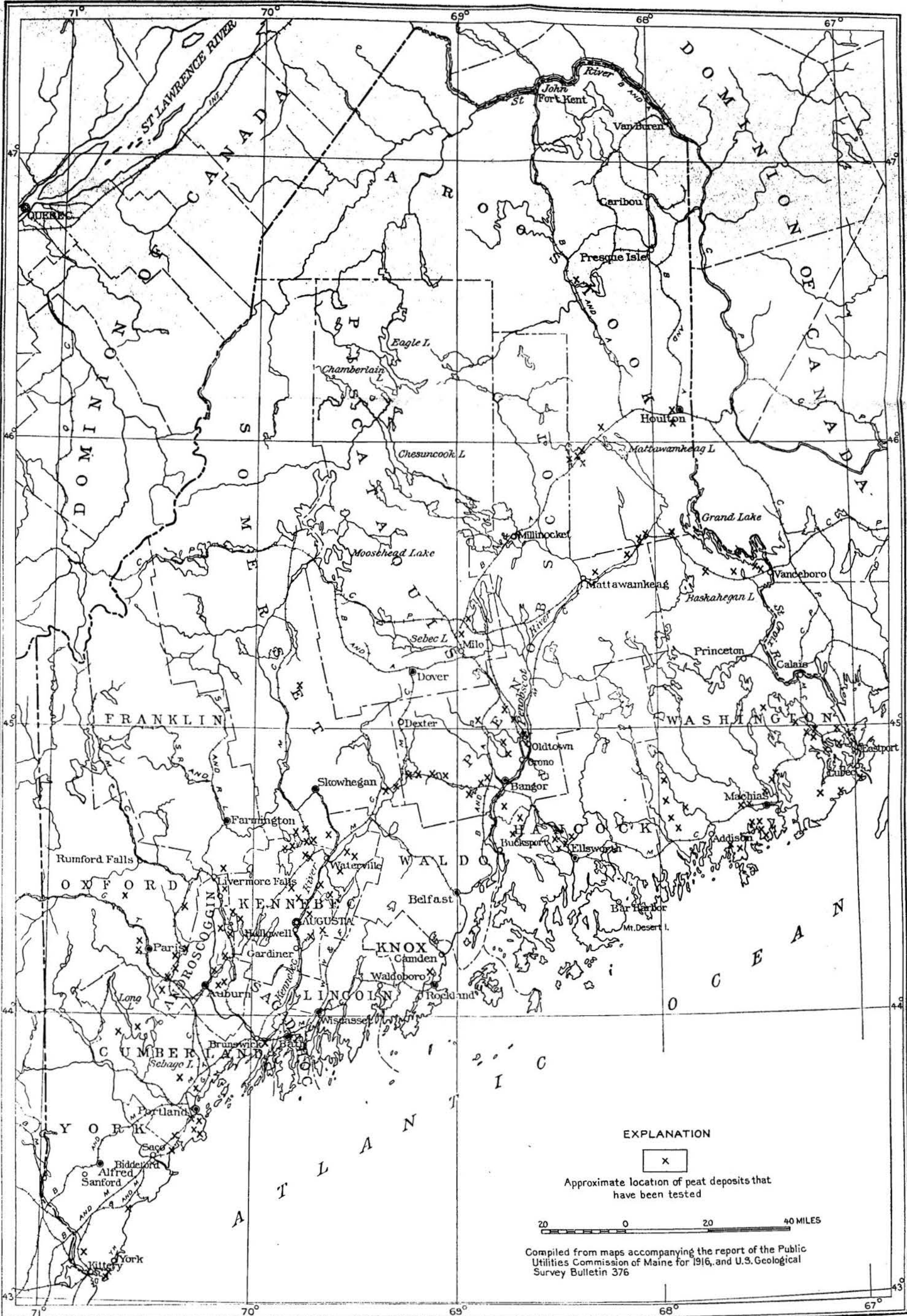



ties, in the northern and western parts of Maine, also contain large deposits of peat, but on account of the lack of transportation facilities in this region little is known concerning the quality of this peat. Bastin and Davis ${ }^{42}$ say that peat resources as great or greater than those of southern Maine occur in the forested lake districts of the northern part of the State. Most of the northern deposits, like many of those in northern Minnesota and Michigan, are situated in heavily forested swamps, containing deep standing water, and attempts will hardly be made to use the peat for many years. According to Burr, ${ }^{48}$ nearly all the peat in Maine that will be used commercially for several years lies between the coast and a line drawn from Houlton to the Rangeley lakes.

The built-up deposit formed on relatively flat or gently sloping land surfaces is the predominant type in Maine and, to the scientist, the climbing bog, a variety of this predominant type, is of most interest. The predominance of built-up peat in Maine is ascribed to the extensive flat, undrained areas and to the cool humid climate, conditions favorable to the growth of sphagnum, heath shrubs, and associated coniferous trees. The largest and deepest of these deposits occur in the northern part of the State. The climbing bogs of Maine are described by Shaler ${ }^{44}$ as follows:

Although bogs are ordinarily formed beneath lake basins by the gradual growth of water-loving vegetation from their sides and bottoms, a process which goes on until the lake may be converted into a normal peat swamp, there is another way in which peat may be accumulated and which in certain regions gives rise to very extensive bogs which were not formed in a water basin; these are commonly known as climbing bogs, and in one or another of their several forms they are widely disseminated. In high latitudes peat deposits are due to the growth in a luxuriant form of the common sphagnum. Starting on the borders of a lake basin, the dense mass of branches of this plant will, if the air be very moist, grow not only over the surface of the water but upward from its level upon slopes which may have an inclination of $5^{\circ}$ or $10^{\circ}$. As the lower part of the vegetable mat decays it forms a peat of ordinary quality, which may gradually attain a thickness of many feet. In the process of growth these highland morasses often extend into forests, where they gradually kill the trees, so that the region once wooded may become an open moorland.

In eastern Canada, Newfoundland, and the Maritime Provinces, climbing bogs of the type so common in Ireland and other portions of northwestern Europe occur, but they lack the extent and depth of those in the Old World. Within the United States the only climbing bogs of a conspicuous character occur in eastern Maine, but even there they are relatively unimportant except from a scientific point of view. Similar accumulations of a smaller sort formed on declivities may be noted in the more western parts of New England and in northeastern New York. Nowhere within the limits of the United

Bastin, E. S.; and Davis, C. A., Peat deposits of Maine: U. S. Geol. Survey Bull. 376 , p. 113, 1909 .

4 Burr, F. F., The peat deposits of Maine : Public Utilities Commission of Maine Second Ann. Rept., p. 71, 1916.

"Shaler, N. S., Origin, distribution, and commercial value of peat deposits: U. S. Geol. Survey Sixteenth Ann. Rept., pt. 4, pp. 308 and 311, 1895. 
States, so far as is known to the writer, are these high-lying bogs of other than scientific interest.

Although some filled-basin peat is found in Maine, relatively few deposits contain only that kind. The deposit on the east shore of Pushaw Lake, about 8 miles north of Bangor, is one of the largest filled-basin deposits. In most places this type underlies built-up peat.

According to Burr ${ }^{45}$ and Bastin and Davis, ${ }^{46}$ a total area of 50 square miles of readily accessible peat 8 feet in average depth has been examined. These deposits are estimated to be capable of yielding $48,000,000$ short tons of air-dried peat. However, in addition to this quantity, there are large bogs and swamps in the northern forested region as well as in relatively inaccessible southern areas that probably contain a quantity as large or larger. It is believed that the peat de. posits of Maine would yield at least 100,000,000 short tons of airdried peat. The following table ${ }^{47}$ gives a brief description of the most readily accessible peat deposits in Maine. Analyses of the peat are given on pages $30-33$.

Peat deposits in Maine.

\begin{tabular}{|c|c|c|c|c|c|c|}
\hline Location. & $\begin{array}{l}\text { Dis- } \\
\text { tance } \\
\text { from } \\
\text { rail- } \\
\text { rood } \\
\text { (miles). }\end{array}$ & Floral condition. & $\begin{array}{c}\text { Area } \\
\text { (acres). }\end{array}$ & $\begin{array}{l}\text { Average } \\
\text { depth } \\
\text { (feet). }\end{array}$ & $\begin{array}{l}\text { Quantity } \\
\text { of air-dried } \\
\text { peat avail- } \\
\text { able (short } \\
\text { tons). }\end{array}$ & Remarks. \\
\hline \multicolumn{7}{|l|}{$\begin{array}{l}\text { ANDROSCOGGIN } \\
\text { COUNTY. }\end{array}$} \\
\hline East Livermore......... & $\begin{array}{l}3 \\
1\end{array}$ & $\begin{array}{l}\text { Forested.............. } \\
\text { Open hesth......... }\end{array}$ & $\begin{array}{l}300 \\
160\end{array}$ & $\begin{array}{r}10 \\
6\end{array}$ & $\begin{array}{l}600,000 \\
250,000\end{array}$ & \\
\hline $\begin{array}{l}\text { Lowiston: } \\
\text { Farwell Bog........ } \\
\text { No Name Bog..... }\end{array}$ & $\begin{array}{l}2 \\
3\end{array}$ & $\mid \begin{array}{l}\ldots . \text { do } \\
\cdots \\
\cdots\end{array}$ & $\begin{array}{l}130 \\
125\end{array}$ & $\begin{array}{l}15 \\
15\end{array}$ & $\begin{array}{l}400,000 \\
400,000\end{array}$ & \\
\hline $\begin{array}{l}\text { Leeds: } \\
\text { West of Center.... } \\
\text { South of Center... } \\
\text { North of Center... }\end{array}$ & $\begin{array}{l}2 \\
2 \\
1\end{array}$ & 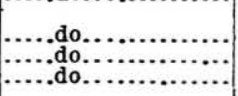 & $\begin{array}{r}100 \\
200 \\
2\end{array}$ & $\begin{array}{r}4 \\
15 \\
20\end{array}$ & $\begin{array}{r}80,000 \\
600,000 \\
8,000\end{array}$ & \\
\hline \multicolumn{7}{|l|}{ AROOSTOOK COUNTY. } \\
\hline 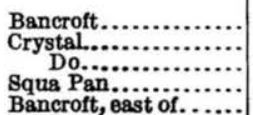 & $\begin{array}{r}2 \\
2 \\
1 \\
1 \\
\text { Near. }\end{array}$ & $\begin{array}{l}\text { Cedar swamp....... } \\
\text { Partly wooded....... } \\
\text { De..do............... } \\
\text { Open heath......... }\end{array}$ & $\begin{array}{r}200 \\
100 \\
60 \\
200 \\
100\end{array}$ & $\begin{array}{c}2(?) \\
5 \\
9 \\
2 \\
10\end{array}$ & \begin{tabular}{|c|} 
Little. \\
100,000 \\
25,000 \\
Little.(?) \\
200,000
\end{tabular} & \\
\hline 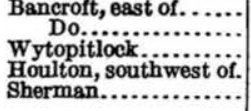 & $\begin{array}{l}\text { Near. } \\
\text { Near. } \\
\text { Near. } \\
\text { Near. } \\
\text { Near. }\end{array}$ & $\begin{array}{l}\text { Open heath .......... } \\
\text { Cedar swomp...... } \\
\text { Wett wooded bog..... } \\
\text { Open hesth........ } \\
\text { Partly wooded...... }\end{array}$ & $\begin{array}{r}100 \\
100 \\
150 \\
30 \\
50\end{array}$ & $\begin{array}{r}10 \\
2 \\
(?)^{4} \\
7\end{array}$ & $\mid \begin{array}{c}200,000 \\
\cdots \cdots \cdots \cdots \cdots \\
\cdots \ldots \ldots \ldots \\
25,000 \\
70,000\end{array}$ & $\begin{array}{l}\text { Not promising. } \\
\text { Do. }\end{array}$ \\
\hline \multicolumn{7}{|l|}{ CUMBERLAND COUNTY. } \\
\hline Brunswick, College & 2 & Shrubby heath...... & 160 & $3-$ & Little. & Local use \\
\hline $\begin{array}{l}\text { Cape Elizabeth......... } \\
\text { Falmouth . ........... } \\
\text { Sebago, Northwest } \\
\text { River. }\end{array}$ & $\begin{array}{r}5 \\
8\end{array}$ & $\begin{array}{c}\text { Part wooded.......... } \\
\text { Š..do.................... }\end{array}$ & $\begin{array}{r}100 \\
85 \\
600\end{array}$ & $\begin{array}{l}10 \\
15 \\
(?)\end{array}$ & $\begin{array}{l}225,000 \\
250,000 \\
(?)\end{array}$ & \\
\hline HANCOCK COUNTY. & & & & & & \\
\hline $\begin{array}{l}\text { Bucksport, Mud Pond. } \\
\text { Nicolin................. }\end{array}$ & $\begin{array}{l}5 \\
1\end{array}$ & 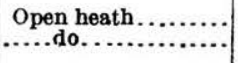 & $\begin{array}{l}20 \\
12\end{array}$ & $\begin{array}{l}10 \\
10\end{array}$ & $\begin{array}{l}40,000 \\
25,000\end{array}$ & \\
\hline FRANKLIN COUNTY. & & & & & & \\
\hline Chesterville........... & 5 & Open heath......... & 300 & 6 & 300,000 & \\
\hline
\end{tabular}


Peat deposits in Maine-Continued.

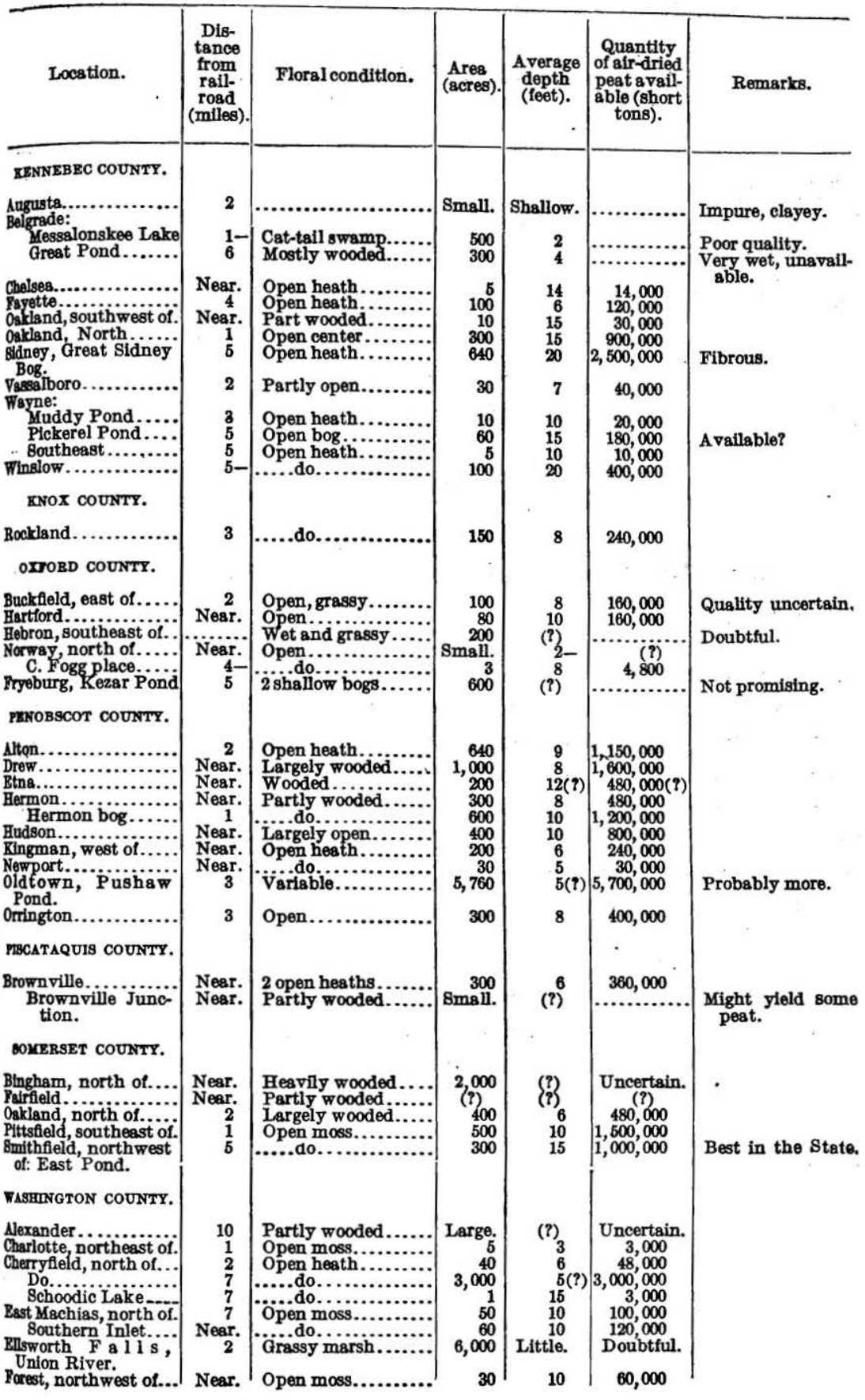


Peat deposits in Maine-Continued.

\begin{tabular}{|c|c|c|c|c|c|c|}
\hline Locstion. & $\begin{array}{l}\text { Dis- } \\
\text { tanoe } \\
\text { from } \\
\text { rail: } \\
\text { rosad } \\
\text { (iniles). }\end{array}$ & Floral condition. & $\begin{array}{c}\text { Area } \\
\text { (acres). }\end{array}$ & $\begin{array}{l}\text { Average } \\
\text { depth } \\
\text { (feet). }\end{array}$ & $\begin{array}{c}\text { Quantity } \\
\text { of air-dried } \\
\text { peat avail- } \\
\text { able (short } \\
\text { tons). }\end{array}$ & Remarks. \\
\hline $\begin{array}{l}\text { Jonesboro: } \\
\text { Whitneyville....... } \\
\text { Pond Cove........ } \\
\text { Roque's Bluff...... } \\
\text { Black Head...... } \\
\text { Jonesport, east of...... } \\
\text { Jonesport, north of.... } \\
\text { Lubec: Quoddy Head. }\end{array}$ & \begin{tabular}{c} 
Near. \\
\hdashline$\cdots . . .$. \\
\hdashline$\cdots . . . \%$ \\
\hdashline$\cdots . . \%$ \\
8 \\
7
\end{tabular} & 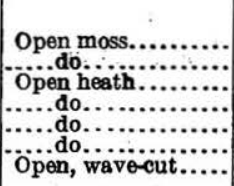 & $\begin{array}{l}20 \\
40 \\
15 \\
10 \\
40 \\
20 \\
20\end{array}$ & $\begin{array}{r}8 \\
5 \\
5 \\
10 \\
10 \\
6 \\
5\end{array}$ & $\begin{array}{l}30,000 \\
40,000 \\
15,000 \\
20,000 \\
80,000 \\
24,000 \\
20,000\end{array}$ & \\
\hline $\begin{array}{l}\text { Pembroke: } \\
\text { Ayers Junction..... } \\
\quad \text { Do.............. } \\
\text { Falls Point............. } \\
\text { Perry ................. } \\
\text { Trescott, south of ..... } \\
\text { Vanceboro, west of.... } \\
\text { Danforth, west of..... }\end{array}$ & $\begin{array}{l}\text { Near. } \\
\text { Near. }\end{array}$ & $\begin{array}{l}\text { Open moss......... } \\
\text { Open moss sedgy.... } \\
\text { Open heath........ }\end{array}$ & $\begin{array}{r}160 \\
50 \\
35 \\
10 \\
160 \\
500 \\
300\end{array}$ & \begin{tabular}{c|c|}
15 \\
5 \\
10 \\
3 \\
8 \\
10 \\
$6(?)$
\end{tabular} & $\begin{array}{c}280,000 \\
50,000 \\
70,000 \\
6,000 \\
250,000 \\
1,000,000 \\
300,000 \text { (?) }\end{array}$ & \\
\hline
\end{tabular}

NEW HAMPSHIRE.

GENERAL FEATURES.

Cheshire, Hillsborough, Rockingham, and Sullivan counties contain the largest and most valuable peat deposits in New Hampshire. Some peat is also found in Belknap and Coos counties. Although there are a few salt-marsh deposits on the coast, notably near North Beach, Greenland, and Hampton Beach, most of the peat originated in fresh-water basins. The deposits of New Hampshire are estimated to be capable of yielding 1,000,000 short tons of air-dried peat, a quantity much less in proportion to the size of the State than is found in the other New England States. This condition is ascribed to the roughness of the land surface and to the lack of extensive poorly drained depressions.

\section{Cheshire coUnty.}

Locality 1.-A deposit 1 to $1 \frac{1}{2}$ miles south of East Jaffrey contains about 50 acres of peat, 6 feet in average thickness, and should yield approximately 60,000 short tons of air-dried peat. (See fig. 15.) The vegetation consists chiefly of spruce, tamarack, and maple trees, heath shrubs, blueberry, sedges, and grasses. A series of test borings gave the following results:

Thickness of peat in test borings A and B in deposit south of East Jaffrey, N. H.

Hole $\dot{A}$, northeast corner of deposit, a short distance south of wagon road: Brown well-decomposed soft plastic peat, consisting chiefly of the remains of sphagnum and heath shrubs but containing some woody roots, stumps of trees, and small spruce and cedar logs

Hole B, about 500 yards southwest of hole A : Peat similar in structure to that found in hole $\mathrm{A}$ 
A composite sample (see analysis 7, p. 43) obtained by taking peat at intervals of 2 feet in depth in each test hole indicates that the peat might make good fuel.

Locality 2.-A deposit about $1 \frac{1}{2}$ miles south of East Jaffrey and onefourth of a mile west of Contoocook Pond, which is largely covered with water as a consequence of the damming of Contoocook River, contains an average thickness of about 3 feet of peat near its eastern margin. (See fig. 15.) The dominant living vegetation consists of spruce and tamarack trees, heath shrubs, the blueberry, and sphagnum moss. Some of the sphagnum is of the large leafy variety and is suitable for use in surgical dressings. Two test borings gave the following results:

Thickness of peat in test borings $C$ and $D$ in deposit south of East Jaffrey, $N . H$.

Hole C, at roadside in center of narrow arm of bog extending eastward toward Contoocook Pond: Brown fibrous peat, composed of the remains of sphagnum and heath shrubs.

Hole D, 40 yards west of hole $\mathrm{C}$ : Brown fibrous peat_______- 3

A composite sample (see analysis 8, p. 43) was obtained by mixing peat taken at intervals of 1 foot in depth in each hole. Although good fiel might be made from the peat, the deposit is too shallow for commercial use.

Locality 3.-A small bog about a quarter of a mile east of Chesham Pond is shown on the map of the Monadnock quadrangle published by the United States Geological Survey. The area of the bog is about 25 acres, and the average depth of the peat is about 3 feet. The peat is of good quality, but the deposit is too small for commercial use. A large quantity of sphagnum was observed at this locality.

Locality 4.-Tenant Swamp, about 1 mile northwest of the railroad sta-

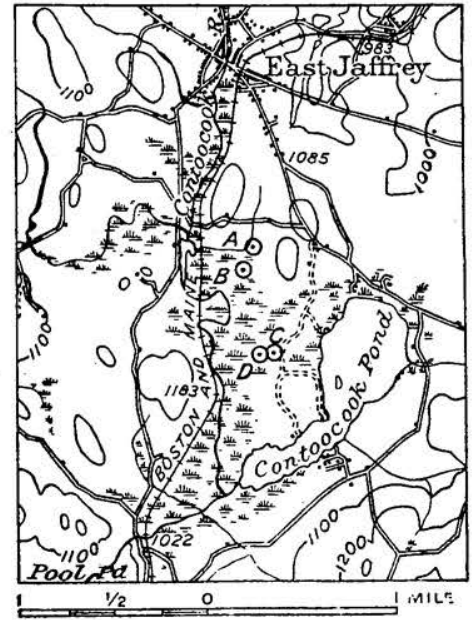

Figure 15.-Map of peat deposits near East Jaffrey, Cheshire County, N. H., showing approximate position of test borings for peat. tion at Keene, occupies the bottom lands along Ashuelot River and is densely overgrown by maple and pine trees and underbrush. This swamp is shown on the map of the Keene quadrangle, published by the United States Geological Survey. A few scattered tamarack and spruce trees and some sphagnum moss grow in the wetter parts of $91085^{\circ}-22-11$ 
the area. Although numerous borings were made, no valuable peat was found. A shallow deposit of black muck covers the surface in some places, but there is practically no accumulation of plant remains in a large part of the area.

\section{HILLSBOROUGH COUNTY.}

Locality 5.-A bog about 1 mile southeast of Ponemah, on the north side of the Boston \& Maine Railroad tracks, is probably about

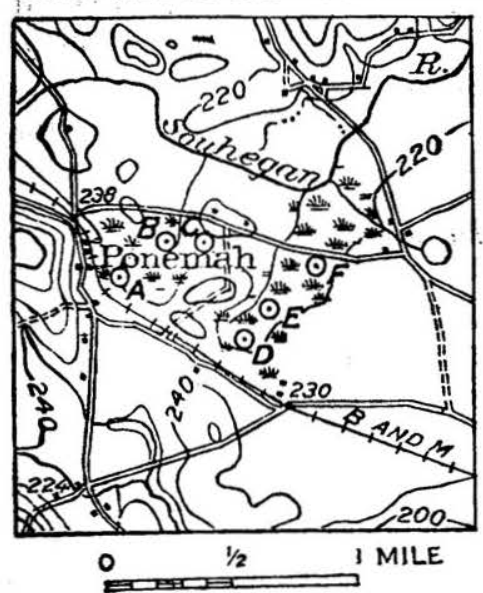

Frgurs 16.-Map of peat deposits near Ponemah, Hillsborough County, N. H., showing approximate position of test borings for peat. 150 acres in area and contains a deposit of peat 9 feet in average depth, so that it should yield approximately 270,000 short tons of air-dried peat. (See fig. 16.) The living vegetation consists chiefly of heath shrubs, sphagnum and polytrichum moss, sedges, cotton grass, and the blueberry. Small spruce trees were once numerous, but they no longer contribute notably to the formation of peat. Although sphagnum moss is present in great quantities in this locality, it is not suitable for surgical dressings. It might, however, be useful for packing material or stable litter. A series of test borings gave the following results:

Thickness of peat in test borings $D, E$, and F, in bog southeast of Ponemah, N.H.

reet.

Hole D, south end of deposit, about 50 yards north of the Boston \& Maine Railroad tracks: Dark-brown well-decomposed soft plastic peat, containing the remains of sphagnum, heath shrubs, and sedges. almost structureless near bottom.

Hole $\mathrm{E}$, about 50 yards northeast of hole $\mathrm{D}$ along a line through center of bog: Peat similar to that in hole A, locality 6

Hole $\mathrm{F}, 50$ yards northeast of hole $\mathrm{E}$ : Peat similar to that in holes $\mathrm{A}$ and $\mathrm{B}$, locality 6

A composite sample (analysis 3, p. 43) consisting of peat taken at intervals of 2 feet in depth from each hole, shows the peat to be of good quality for fuel or fertilizer. This bog could be easily drained.

Locality 6.-A bog east of Ponemah and north of the tracks of the Boston \& Maine Railroad contains an open-meadow deposit consisting of pond peat overlain by built-up peat. (See fig. 16.) The bog is about 100 acres in area, and the peat is 16 feet in average thickness. The deposit appears to be capable of yielding approximately 340,000 
short tons of air-dried peat and muck. The living vegetation consists chiefly of grasses, sedges, ferns, sphagnum, and polytrichum moss. A series of test borings gave the following results:

Thickness of peat in test borings $A, B$, and $C$ in bog east of Ponemah, $N$. $H$.

Hole A, about 150 yards from west side of bog, near drainage ditch :

Black muck

Feet.

Brown rather fibrous grass-edge peat

1

Fibrous woody peat

3

Brown well-decomposed soft plastic peat

Thoroughly decomposed peat

Gray fine-grained soft pond peat, somewhat clayey

Hole B, about 150 yards east of hole A, near drainage ditch:

Muck and peat similar to that in hole $A$

Hole C, about 150 yards east of hole B, near drainage ditch: Muck and peat similar to that in holes $\mathbf{A}$ and $\mathbf{B}$.

A typical sample (see analysis 4, p. 43) was obtained by mixing muck and peat taken at intervals of 2 feet in depth from each of the test holes. This sample contains a large proportion of ash, a condition that may be due to the clayey material from the bottom of the deposit or to inorganic impurities mixed with the peat in the excavation of the drainage ditch along which the test holes were made. The upper layers of the peat probably contain less ash than is shown by the analysis, and they may be suitable for fuel. This bog adjoins a railroad, is practically treeless, and could be easily drained. The peat in the upper portion of the deposit appears to be of good quality for fuel or fertilizer.

Locality 7.-A bog between the wagon road and the tracks of the Boston \& Maine Railroad about 1 mile south of South Milford, which is shown on the map of the Milford quadrangle published by the United States Geological Survey, lies in an open meadow overgrown chiefly by sphagnum, cotton grass, sedges, the cranberry, and polytrichum moss. The sphagnum, much of which is of the large leafy type, suitable for surgical dressings, is dominant. A series of test borings gave the following results:

Thickness of peat and muck in test borings in bog near South Milford, N. B.

Hole $A$, near east margin of bog adjoining wagon road: Brown, partly decomposed peat, made up of sphagnum, grass, and sedge remains, underlain by sand__._-_._-_ 1

Hole B, west of hole A, in center of bog: Muck

Analysis 5 (p. 43) shows the composition of a sample obtained from the two test holes. 
Locality 8.-A large swamp extends southwest from the railroad station at Greenfield for a distance of about 2 miles. Localityis includes an arm of this swamp about an eighth of a mile west: of Greenfield. The total area of the swamp, which is shown on the map of the Peterboro quadrangle published by the United States Geological Survey, is about 640 acres, but the area of the small arm included in this locality is only about 20 acres. In earlier years the entire swamp was covered by a dense growth of trees, but the area designated locality 8 has been cleared of trees and is overgrown by grasses, sedges, sphagnum moss, and the blueberry. The sphagnum is not well developed. Several test holes were put down along a line through the center of the locality, and a representative sample was obtained. The deposit is only about $3 \frac{1}{2}$ feet thick where tested, and the peat as represented by analysis 6 (p. 43) is of poor quality for fuel. Although the peat is deeper in the center of the swamp, that portion is covered with so dense a growth of large trees and underbrush that it could not be profitably cleared for the production of peat.

Locality 9.-Locality 9 includes another arm of the swamp near Greenfield in which locality 8 is situated. The area consists of about 10 acres adjoining a road that extends southward from Greenfield. The living vegetation consists chiefly of sphagnum, sedges, and grasses. The average thickness of the peat, which is fibrous, silty, and unsuited for fuel, is only 2 feet.

\section{ROCKTNGHAX COUNTY.}

Locality 10.-A salt marsh which adjoins Great Bay, about 1 mile northwest of Greenland, and which is shown on the map of the Dover quadrangle, published by the United States Geological Survey, covers about 640 acres. Three test holes were put down in the marsh at intervals of about 100 yards, starting about 200 yards north of the tracks of the Boston \& Maine Railroad. The deposit consists of gray soft clayey material, containing the undecomposed remains of marsh grasses. A strong odor of hydrogen sulphide was noticed when each hole was put down. The average thickness of the deposit is about 9 feet. At a depth of 7 feet a thin layer of logs and pieces of wood was encountered. The wood was well decomposed and soft and was easily penetrated by the sampling rod. Analysis 1 (p. 43) shows that the deposit contains muck and is too impure for economic use.

Locality 11.-Packer Bog, about half a mile south of Greenland, which adjoins Packer Brook, is overgrown by maple, poplar, and ash trees and contains from 6 to 8 inches of black muck. A little sphagnum moss was seen, but it appeared to be unsuitable for surgical dressings. No valuable peat was found and hence no samples were taken. 
Locality 12.-Great Bog, about 1 mile northeast of Greenland station, is similar in character to Packer Bog.

Locality 13.-In eight small marshes south and southeast of Portsmouth no valuable peat was found.

Locality 14.- Hampton Marsh is an extensive salt marsh along the coast of New Hampshire in the vicinity of Northampton, Hampton Beach, and Seabrook. It is several square miles in area, and the peat where tested ranges in thickness from 1 to 15 feet. The deposit is a typical New England salt marsh containing impure peat and muck. The peat is of no economic value.

Locality 15.-A deposit on the Boston \& Maine Railroad near Powow Station, which is shown on the map of the Haverhill quadrangle, published by the United States Geological Survey, is covered with deep water and the peat is inaccessible without extensive drainage.

Locality 16. - Spruce "Swamp," east of Fremont, is about 640 acres in area, but not more than 300 acres is covered with workable peat. (See fig.

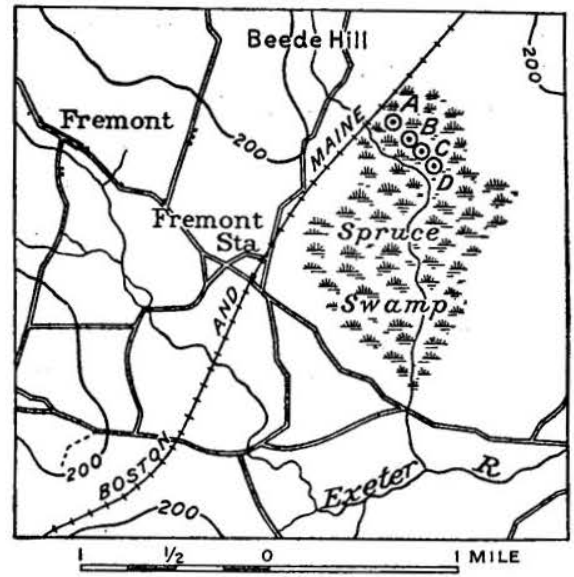

Frgure 17.-Map of Spruce Swamp, Rockingham County, N. H., showing approximate position of test borings for peat.

17.) The peat where sampled is 4 to 5 feet in average thickness and is underlain by sand and clay. If there are 200 acres of peat 4 feet in average depth, the deposit should yield about 160,000 short tons of air-dried peat. The living vegetation consists of small broad-leafed trees, heath shrubs, sphagnum, and polytrichum moss, and other small plants. Much of the sphagnum moss might be used for surgical dressings. A series of test borings gave the following results:

Thickness of peat in Spruce Swamp, east of Fremont, N. H.

Hole A, at north end about 200 yards east of point where railroad track crosses stream flowing through bog: Brown welldecomposed soft plastic sphagnum peat, containing a few roots and pieces of wood.

Hole B, about 100 yards southeast of hole A: Peat similar to that found in hole $A$

Hole C, about 100 yards southeast of hole B: Same character of material as that in hole $B$.

Hole D, about 100 yards southeast of hole C: Peat similar to that found in hole A 
A composite sample (analysis 2, p. 43 ) was obtained by combining samples of peat taken at intervals of 1 foot in depth from each of the four test holes. The deposit appears to contain peat of good quality, but it is doubtful, on account of surface conditions, whether peat could be economically produced.

\section{OTHER OOUNTIES.}

Other small deposits are known in Belknap, Coos, Merrimack, Strafford, and Sullivan counties, but only a few of these contain peat of good quality for fuel or fertilizer. It is reported that peat fuel has been made and sold from a small bog near Hooksett, Merrimack County, but the deposit is not now worked.

VERMONT.

GENERAL FEATURES.

Despite the mountainous surface of a large part of Vermont, the State contains numerous widely distributed peat deposits of all types except those found in salt marshes. The deposits that offer the most promising possibilities of development are those in Bristol Pond and Shoreham Swamp, Addison County; near South Burlington, Chittenden County; Franklin, Highgate, and Richford, Franklin County; and North Hero, Isle la Motte, and South Hero, Grand Isle County. Other large areas of peat are found in these counties as well as in Orleans and Windham counties. It is estimated that $8,000,000$ short tons of air-dried peat could be obtained from the deposits in Vermont.

The localities described below were tested for peat during the progress of the field work upon which this report is based. Other detailed data relating to the peat deposits of Vermont may be consulted in a report issued by the Vermont Agricultural Experiment Station..$^{48}$

\section{ADDISON COUNTY.}

Locality 1.-A large swamp on the west side of Otter Creek, about 1 mile west of Salisbury, which is shown on the map of the Brandon quadrangle, published by the United States Geological Survey, is about 2 square miles in area and the deposit is 10 feet in average thickness. The material in the upper 3 feet and the lower 2 feet is not suitable for fuel. If the average thickness of the peat suitable for fuel is 5 feet, the bog should yield about 1,280,000 short tons of air-dried machine peat. The dominant living vegetation consists of cedar, red maple, poplar, birch, and oak trees. A series of test holes gave the following results:

is The peat and muck deposits of Vermont: Vermont Agr. Exper. Sta. Bull. 165, 1912. 


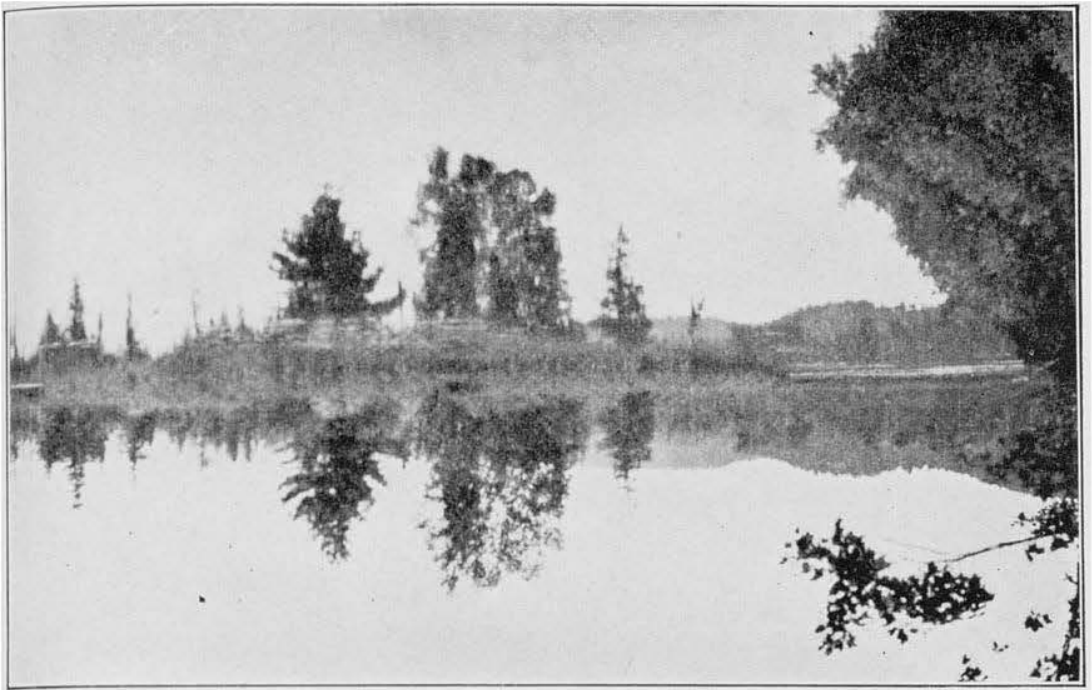

A. BRISTOL POND, ADDISON COUNTY, VT.

Shows floating mat of vegetation near shore and spruce trees in the background.

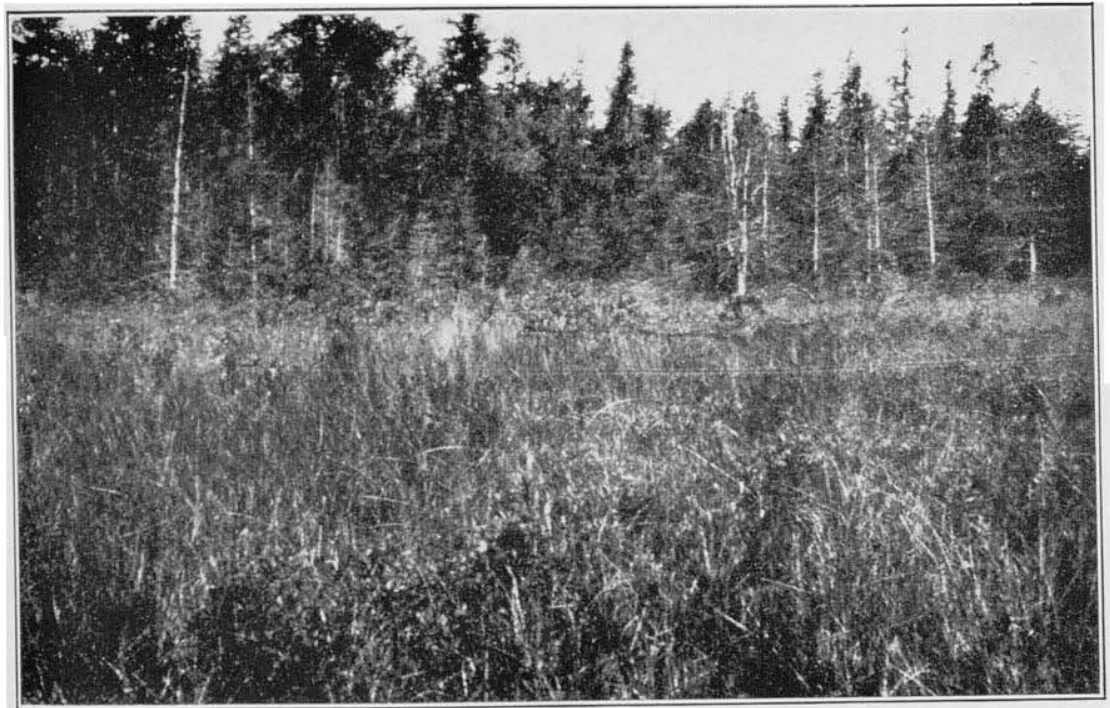

B. SPRUCE-SPHAGNUM BOG ON THE SHORE OF BRISTOL POND.

The Evolution of a Peat Bog. 

Log of test borings in swamp on Otter Creek, 1 mile west of Salisbury, Vt.

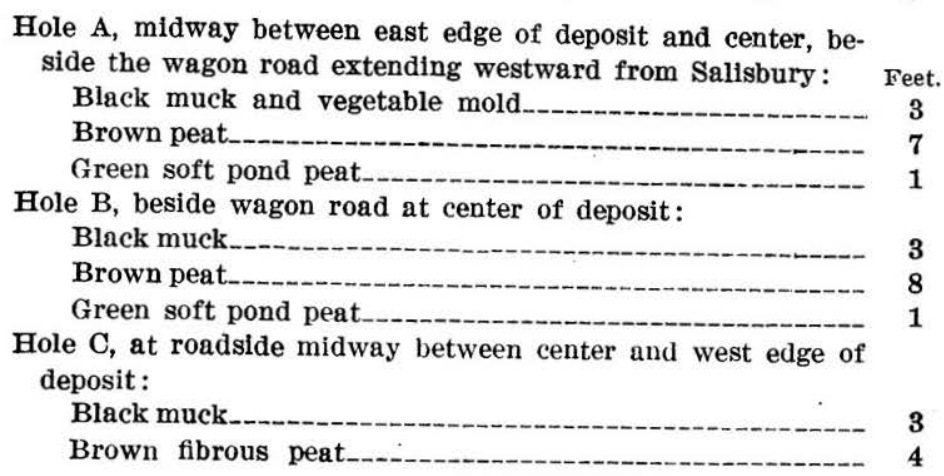

Samples were taken from each of the test holes at intervals of 2 feet in depth, beginning 3 feet below the surface and extending to the pond peat at bottom, and were mixed to obtain a typical specimen of the workable peat. Samples of the overlying black muck were also taken. Analysis 9 (p. 49) shows the quality of the workable peat and analysis $9 \mathrm{a}$ that of the muck.

Locality 2.-A deposit east of locality 1 in an open meadow, which was once densely wooded, is about 600 acres in area and contains peat 2 feet deep overlain by about 3 feet of black muck. The land surface is only a few feet above the water level in Otter Creek and is therefore subject to overflow during periods of high water. The living vegetation consists chiefly of grasses, and the peat is brown, well-decomposed, and plastic and contains considerable silt. One test hole was put down midway between Otter Creek and the eastern edge of the wooded portion, beside a wagon road extending westward from Salisbury. Analysis 10 shows the composition of the sample taken from this test hole. The deposit consists chiefly of muck and peat that is too shallow and too silty to be used for fuel.

Locality 3.-A bog west of Bristol Pond (see fig. 18) contains about 500 acres of peat that ranges in thickness from 5 to 13 feet. If the average depth of the workable peat is 6 feet the deposit should yield 600,000 short tons of air-dried peat. This locality is a typical tamarack-spruce-sphagnum bog formed on the western shore of Bristol Pond, the remnant of a large lake. The dominant vegetation consists of tamarack, spruce, and cedar trees, shrubs of the heath family, and sphagnum moss. Sedges, grasses, and ferns are also abundant. The floating mat of vegetation near the shore of Bristol Pond indicates the manner in which the outlying bog was formed. (See Pl. XIV, $A$ and $B$.) From the surface to a depth of 5 to 6 feet the peat is made up of dark-brown to reddish-brown fibrous, slightly decomposed remains of sphagnum moss. The under- 
lying peat is soft, plastic, and well decomposed. Two test holes gave the following results:

Logs of test borings in bog west of Bristol Pond, Addison County, $\nabla t$.

Hole A, midway between west central margin of pond and west edge of bog:

Dark-brown fibrous sphagnum peat

Feet.

Greenish soft pond peat

6

Hole B, one-fourth of a mile south of hole A :

Dark-brown fibrous sphagnum peat.

Greenish soft pond peat

7

Anąlysis 11 (p. 49) represents a composite sample from the upper part of the deposit, which contains brown fibrous sphagnum peat.

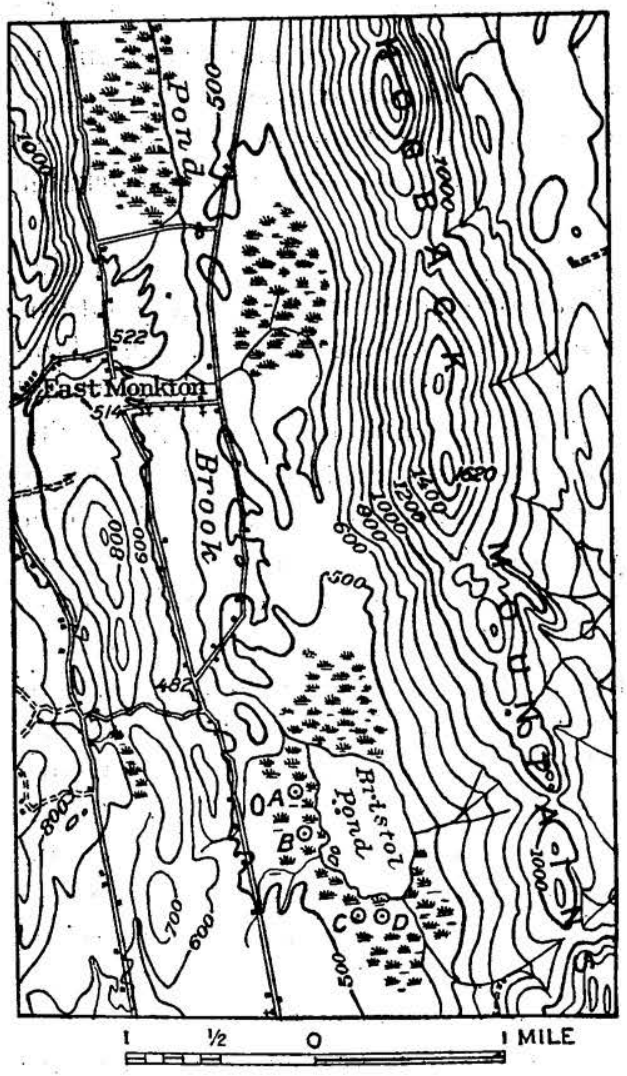

FigURn 18.-Map of peat bogs near Bristol Pond and Pond Brook, Addison County, Vt., showing approximate position of test borings for peat.
It indicates that this peat is suitable for fuel or fertilizer. Analysis 11a shows that the underlying pond peat contains too much ash for use as fuel.

Locality 4.-A bog south of Bristol Pond (see fig. 18) contains peat similar to that in the bog west of Bristok Pond at locality 3 , but not: so deep. Holes $C$ and $D_{4}$ put down near the south shore of the pond, gave the; following results:

Log of test borings in bog south of Bristol Pond, Addison: County, Vt.

Brown fibrous spnagnum peat.

Greenish soft fine-grained pond peat

Feet.

Analysis 12 (p. 49) shows. the character of a composite sample obtained by mixing: peat taken from these borings. It shows that the peat may be valuable for fuel or fertilizer. Although the Bristol bog is one of the largest and best peat deposits in Vermont, it is not very well situated with respect to transportation facilities 
and market and would be difficult to drain. Sphagnum moss, some of which is suitable for surgical use, is very abundant in this locality:

Locality 5.-A bog about 1 mile northeast of East Monkton . (see fig. 18) contains a deposit which was not tested, but it is reported to contain peat 6 feet deep similar in character to that which surrounds the Bristol Pond. The area of this deposit is about 160 acres, and if the average thickness of the peat is 6 feet the deposit is capable of yielding approximately 192,000 short tons of air-dried peat.

Locality 6.-A bog about 2 miles east of Monkton and 1 mile north of East Monkton (see fig. 18) contains about 250 acres of peat, which is reported to be 5 feet deep and to be similar in quality to that

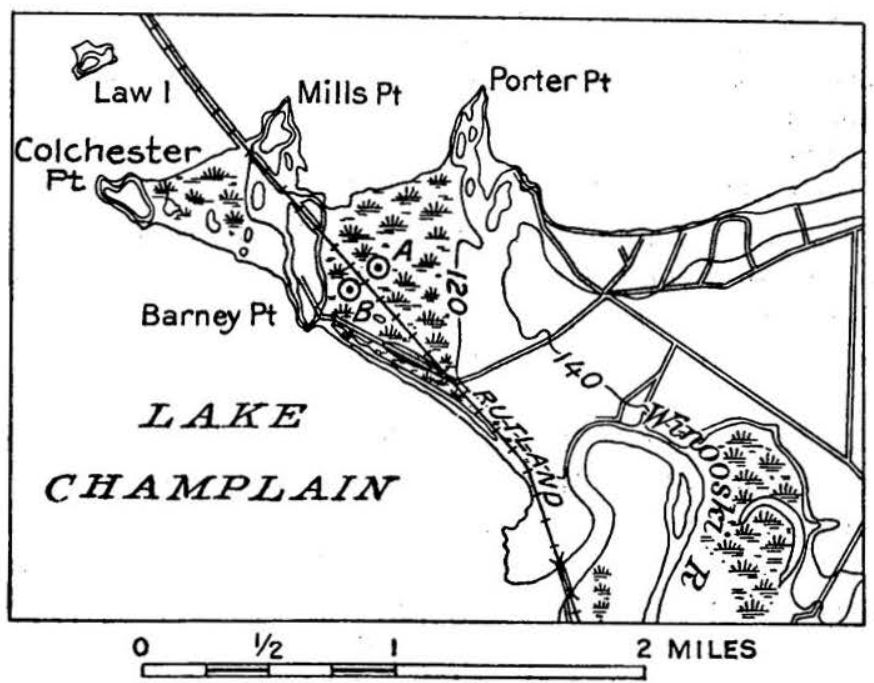

Frodre 19.-Map of peat deposit southwest of Porter Point, Chittenden County, Vt. showing approximate position of test borings for peat.

which surrounds the Bristol Pond. If the average thickness is 5 feet the bog should yield abaut 250,000 short tons of air-dried peat.

\section{CHITTENDEN COUNTY.}

Locality 7.-A marsh about 1 mile south of Malletts Bay and 5 miles north of Burlington, which is shown on the map of the Milton quadrangle, published by the United States Geological Survey, forms an open grassy meadow, about 640 acres in area, used for grazing. The surface is covered with black muck from 1 to 3 feet deep, but no valuable peat was found.

Locality 8.-A deposit near Porter Point, about 7 miles northwest of Burlington (see fig. 19), which is crossed by the tracks of the Rutland Railroad and is skirted on its south side by a wagon road, was formed by the filling of an arm of Lake Champlain. It is 
about 160 acres in area and 12 feet in average thickness and should yield approximately 416,000 short tons of air-dried peat. The living vegetation consists chiefly of tamarack, black spruce, and cedar trees, and heath shrubs, ferns, grasses, and sedges. The peat varies in texture from fibrous to plastic and is made up chiefly of the remains of sphagnum, heath shrubs, grasses, and sedges. Two test holes gave the following results:

Logs of test borings in bog near Porter Point, about 7 miles northwest of Burlington, $V t$.

Hole A, in center of deposit, about 15 yards east of railroad track:

Feet.

Fibrous brown moss and grass peat_____-____-_-_ 8

Well-decomposed to plastic pond peat-_-_-_-_-_-- 5

Hole B, opposite hole A, about 15 yards west of railroad track:

Fibrous brown peat

Soft plastic fine-grained pond peat

Peat was taken at intervals of 2 feet in depth from each hole and mixed to obtain a composite sample. Analysis 13 (p. 49) gives the composition of this sample and shows that the peat is low in ash and high in nitrogen and therefore might be used for fuel or fertilizer.

Locality 9.-A marsh at the mouth of Lamoille River, which is shown on the map of the Milton quadrangle published by the United States Geological Survey and which is about 800 acres in area, was formed by the overflow of water from Lake Champlain and Lamoille River. The depth of the water ranges from 1 to 3 feet during most of the year. Cat-tails, reeds, grasses, and amphibious sedges are the dominant forms of plant life. Black muck from 1 to 3 feet thick was found. Analysis 14 (p. 49) gives the composition of a sample of this muck and shows that, although valueless for fuel, it may be of some value in agriculture.

\section{FRANKIIN COUNTY.}

Locality 10.-A large marsh and swamp about 2 miles southeast of Lakewood is shown on a map of the St. Albans quadrangle published by the United States Geological Survey. The margin of the deposit is overgrown by trees, and the interior is a large open marsh, covered with standing water from 2 to 5 feet deep. The marginal vegetation consists chiefly of red maple trees, and the open marsh is overgrown by cat-tails, sedges, and grasses. The outer forested zone contains no valuable peat. The peat in the open marsh, which is about 500 acres in area, was inaccessible at the time of the writer's visit, but the area is said to contain deep peat of good quality. If the average thickness of the peat in this area is 6 feet, the deposit 
should yield 600,000 short tons of air-dried peat. As drainage would be difficult the deposit is not a promising one for the production of peat.

Locality 11.-A swamp and marsh at locality 11 on the east side of Missisquoi River contains no peat in the outer zone, but the interior was not tested because of excessive surface water.

Locality 12.-A deposit on the Willard farm, about 3 miles from Franklin, which is about 50 acres in area, has been used by John Webster, of Franklin, Vt., for the production of peat fuel. In a report by the Vermont Agricultural Experiment Station ${ }^{40}$ the general features of the deposit and of Webster's early experiments are described substantially as follows:

As indicated by the excavation the peat is black in the upper layers, brown in the center, and dark brown at the bottom. The deposit is about 6 feet deep and overlies a bed of shell "marl." The drainage ditches, which were 7 to 8 feet wide and spaced at intervals of a few rods, were made by Webster in return for the peat excavated. It was found that when the peat was disintegrated and molded, it dried much sooner than cut peat. The first year he sold $\$ 50$ worth of peat fuel, but the next year he was unable to market this quantity because his customers found that the ash pan in the wood stoves used by them was not large enough to hold the ashes that accumulated in even half a day.

The following analysis shows the composition of the peat of this deposit:

Analysis of moisture-free sample of peat from deposit near Franklin, $\nabla t .^{50}$

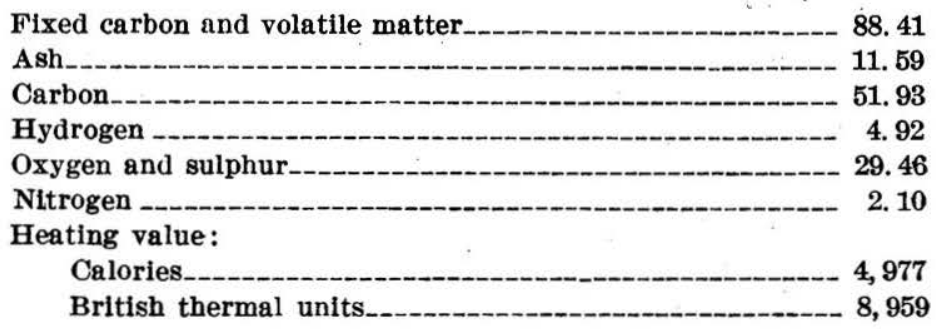

GRAND ISLE COUNTY.

Locality 13.-A swamp that extends from South Hero about 1 mile southward along the Rutland Railroad (see fig. 20) is overgrown by maple, elm, and oak trees. Although the surface is covered by about 1 foot of black muck, the area appears to contain no valuable peat.

Locality 14.-Pearl Swamp, which extends about a mile southward from Grand Isle station (see fig. 20), is about 500 acres in area and is overgrown by maple, elm, butternut, and other trees. Although there is from 6 to 18 inches of black muck on the surface there

Hills, J. L., and Hollister, F. M., The peat and muck deposits of Vermont: Vermont Agr. Exper. Sta. Bull. 165, pp. 186-187, 1912.

${ }^{\infty}$ Hills, J. L., and Hollister, F. M., op. cit., p. 209. 
appears to be no valuable peat in this swamp. Analysis 15 (p. 49) shows the quality of the muck.

Locality 25.-A swamp which extends a mile northward from North Hero station is densely overgrown by maple trees and appears to contain no peat of commercial value.

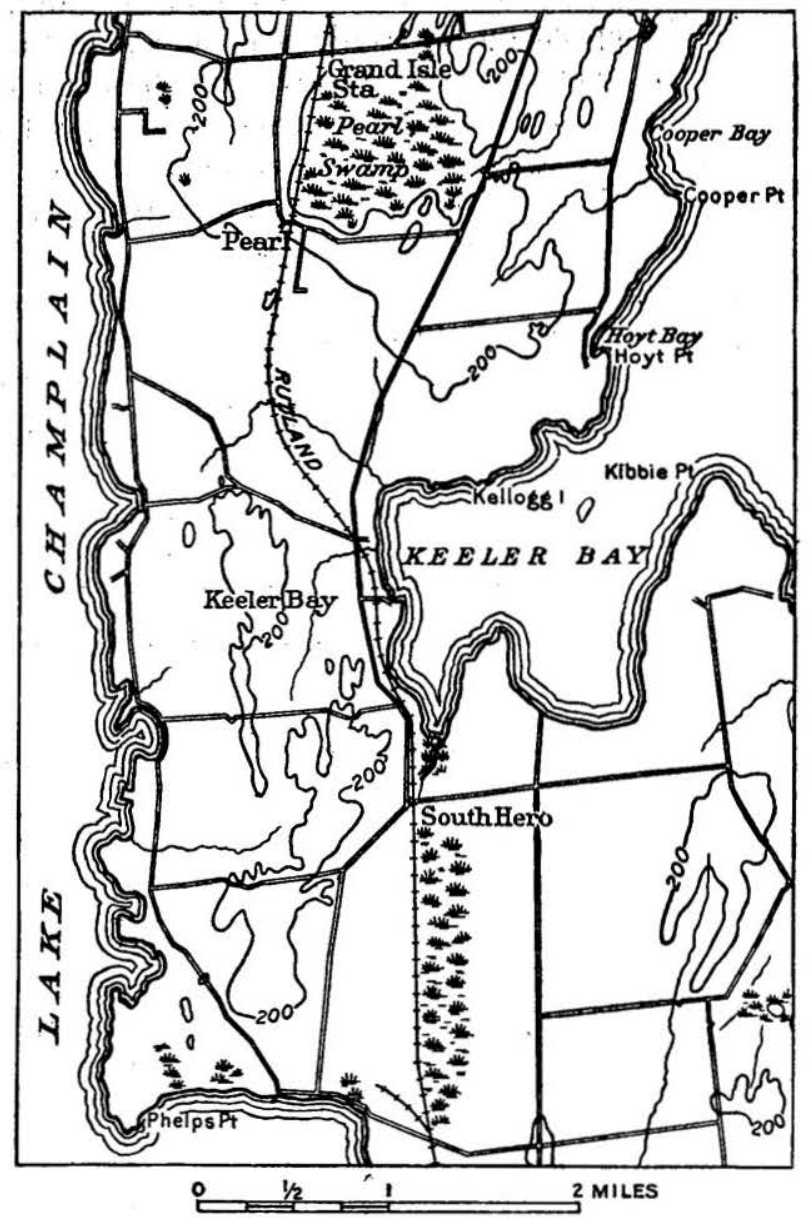

Frgure 20.-Map showing swamps near South Hero and Grand Isle station, Grand Isle County, Vt.

Locality 16.-A large sphagnum-spruce bog that extends northward and southward from Isle La Motte station (see fig. 21) contains a deposit of peat about 1,000 acres in area and 11 feet in average thickness, which should be capable of yielding about $2,200,000$ short tons of peat. The peat, which is composed chiefly of the remains of sphagnum, heath shrubs, sedges, and grasses, is well decomposed, plastic, and of homogeneous texture except the upper layer, which is fibrous. The dominant vegetation consists of spruce 
and tamarack trees, heath shrubs, and sphagnum. Much of the sphagnum is suitable for surgical use. A series of test borings gave the following results:

Logs of test borings in bog near Isle La Motte station, Vt.

Hole A, 100 yards west of east edge of deposit, on south side of road:

Brown flbrous sphagnum moss, slightly decomposed 2

Brown flbrous sphagnum peat

Dark-brown well-decomposed, soft plastic peat

Hole B, halfway across bog near roadside:

Brown flbrous sphagnum moss

Brown flbrous sphagnum peat

Dark-brown well-decomposed, soft plastic peat

Beet.

Hole C, near roadside, 100 yards west of hole B :

Brown flbrous sphagnum moss

Dark-brown sphagnum peat.

Dark-brown well-decomposed soft plastic peat

Light-brown plastic grass-sedge peat

Analysis 16 (p. 49), which shows the character of a composite sample of peat taken from these holes, indicates that the peat is of good quality. The Isle La Motte bog is the largest and best peat deposit in Vermont, is well located, and could be readily drained.

Locality 17.-A swamp about $1 \frac{1}{2}$ miles south of Alburg (see fig. 22) occupies a flat, undrained area that is densely overgrown by maple and elm trees. There is no valuable peat in the swamp, though the surface consists of black muck. If drained and cleared the land might be of agricultural value.

Locality 18.-A swamp about 12,000 acres in area begins about a mile northwest of Alburg and extends westward along the shore of Lake Champlain to Windmíll Point and Kelly Bay. (See fig. 22.) This swamp, which is skirted on the south by the tracks of the Rutland Rail-

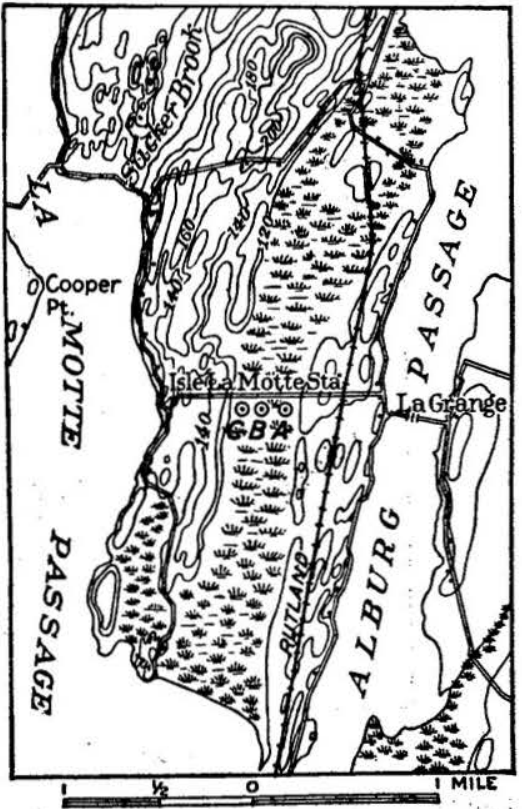

Figurb 21.-Map of bog near Isle La Motte station, Grand Isle County, Vt., showing approximate position of test borings for peat. road, is densely overgrown by maple trees. It appears to contain no peat. 
Locality 19.-A deposit about $1 \frac{1}{2}$ miles east of Alburg (see fig. 22) is about half a mile wide and 4 miles long and extends along both sides of Mud Creek from its mouth at Ransoms Bay, northward into Canada. It is crossed by the tracks of the Central Vermont Railway about a mile north of the mouth of Mud Creek. The area is overgrown by cat-tails and trees and is frequently flooded by Mud Creek. The peat is dark brown and well decomposed and consists chiefly of the remains of marsh grasses. A series of test borings gave the following results:

Logs of test borings in deposit $1 \frac{1}{2}$ miles east of Alburg, V't.

Hole A, 50 yards north of railroad tracks and 100 yards west of creek: Brown silty well-decomposed peat, overlain by 6 inches of black muck and underlain by clay

Hole $B, 50$ yards north of rallroad track and 100 yards east of creek: Peat similar in character to that found in hole A-- 5

A composite sample was obtained by mixing peat taken at intervals of 1 foot in depth from both holes. Analysis 17 (p. 49) gives

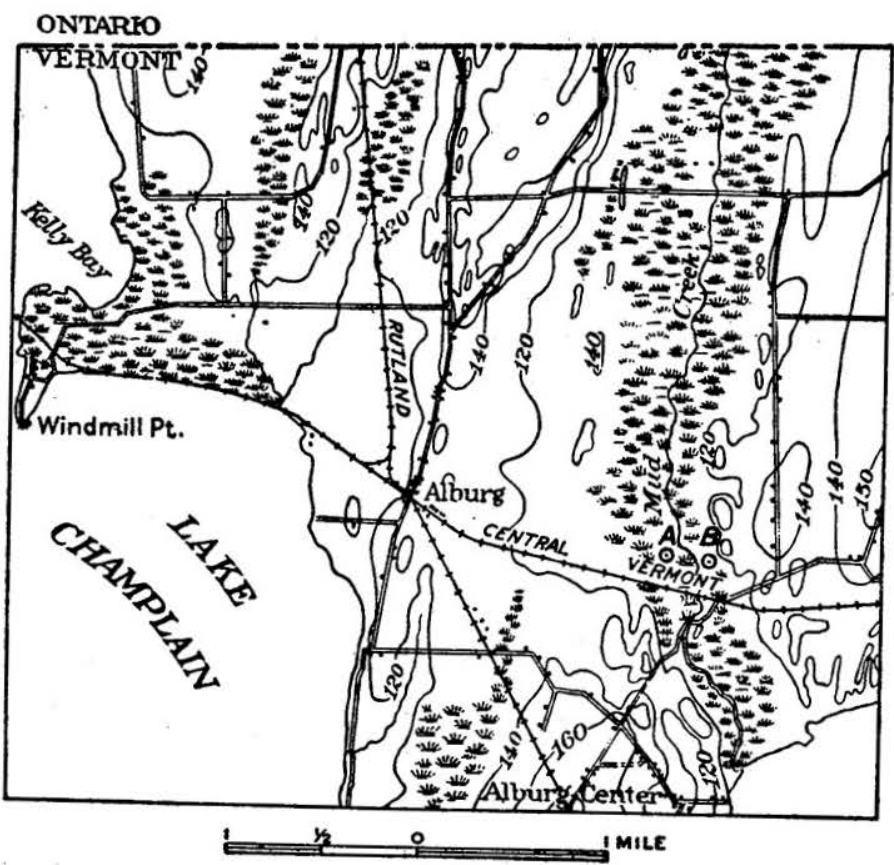

FIGURe 22.-Map of swamp and marsh near Alburg and Kelly Bay, Grand Isle County, Vt., showing approximate position of test borings for peat.

the composition of this sample and shows the peat to be low in ash and high in nitrogen and therefore to be well adapted for use either as fuel or fertilizer. 
RUTLAND COUNTY.

Locality 20.-A spruce swamp about a mile. southwest of Fair Haven is shown on the map of the Whitehall quadrangle published by the United States Geological Survey. The swamp is covered with a dense growth of spruce, tamarack, and cedar trees and scattered maples, alders, and willows. There is no valuable peat in this locality, and the black muck on the surface, which is only a few inches thick, is underlain by sand.

Locality 21.-A swamp east of Fair Haven and north of Castleton River occupies the bottom lands of the river and is therefore annually flooded by it. Maple is the predominant growth. There is no peat in this locality.

Locality 22.-Brandon Swamp, which is about 2 miles northwest of Brandon, between Otter Creek and the tracks of the Rutland Railroad, is shown on the map of the Brandon quadrangle published by the United States Geological Survey. A dense growth of maple, poplar, and birch trees covers the area. No valuable peat occurs in this locality.

\section{MASSACHUSETTS.}

GENERAL FEATURES.

Massachusetts possesses a larger quantity of valuable peat than any other New England State except Maine. The largest and best deposits are those in Essex, Middlesex, and Norfolk counties, the most notable being those near Norwood, East Lexington, Shirley, Littleton, Carlisle, Lawrence, Bedford, and Boston. Small cranberry bogs also occur in Plymouth County and in the Cape Cod region, and salt marshes abound along the coast, but the peat in these areas is of little value. Aside from the salt marshes, which were formed by coastal subsidence and wave action, the peat deposits of Massachusetts originated in glacial depressions and may be placed in the three classes described on pages 9 and 10 . The most typical filled-basin deposits are found in the western part of Middlesex County and the eastern part of Worcester County. The Great Cedar Swamp near Taunton, the meadows near Norwood, and the marshes adjoining Charles River near Boston are the best-developed built-up deposits. The deposits in Massachusetts are estimated to be capable of yielding $12,000,000$ short tons of air-dried peat. The localities described below contain the largest and most accessible deposits in the State. Small and comparatively inaccessible bogs are well distributed throughout the State, but, though valuable for local use, they are of little commercial importance.

BERKSHIRE COUNTY.

Locality 1.-A deposit about 3 miles north of North Adams, which is owned by George W. Hall, is shown on the map of the Greylock 
quadrangle, published by the United States Geological Survey. It is said to be about 25 acres in area and from 6 to more than 25 feet in depth and has been partly cleared. The peat, which is of good quality, is being sold locally at $\$ 10$ a ton. The deposit was not visited by the writer, but information and samples were supplied by the owner. Analysis 48 shows that the percentage of ash is only 9.14 and that of nitrogen 2.62 .

\section{ESSEX AND SUFYOLK COUNTIES.}

Locality 2.-Salt marshes near Revere Beach, adjoining the mouth of Saugus and Pines rivers (see fig. 23), are several square miles in area and contain muck about 10 feet in average depth. The surface of these deposits, which were formed from salt-marsh grasses, lies at

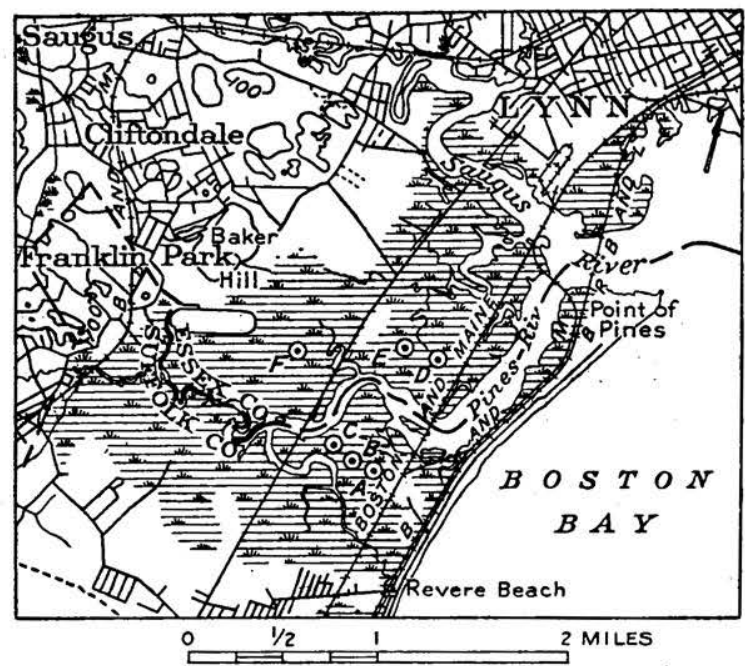

FigURi 23.-Map of salt marshes near Revere Beach, Essex and Suffolk countles, Mass., showing approximate position of test borings for peat.

about high-tide level. The dominant vegetation consists of saltmarsh grasses. The muck is very fibrous and contains a large proportion of clay and silt. A series of test holes gave the following results :

Logs of test holes in salt marshes near Revere Beach, Mass.

Hole A:

Feet.

Brown fibrous, spongy muck, containing undecomposed plant remains

Brown soft plastic silty muck.

Hole B :

Brown very fibrous, spongy muck, containing undecomposed plant remains____._____ 3

Brown soft plastic silty muck 5

Grayish clayey plastic muck 2 
Hole C:

Feet.

Brown very fibrous, spongy muck, containing undecomposed plant remains

Brown soft plastic silty muck

Grayish clayey plastic muck

5

Holes D, E, and F: Muck similar in character and depth to that found in holes $\mathbf{A}, \mathbf{B}$, and $\mathbf{C}$.

A composite sample (see analysis $45, \mathrm{p}$. 34 ) obtained by taking muck at intervals of 2 feet in depth from each of the test holes show the general chemical composition of the deposit. Analysis 45a (p. 34) shows the composition of the upper 3 feet.

Locality 3.-Wenham Swamp, in Topsfield, Wenham, and Hamilton, extends along both shores of Ipswich River. It is shown on the map of the Salem quadrangle published by the. United States Geological Survey. Large elm, poplar, and maple trees are the dominant plant forms. About 500 acres in the center of the swamp is overlain by peat, the average thickness of which is about 8 feet. This peat is black, well decomposed, fine grained, soft, and plastic, and is made up chiefly of the remains of trees. Buried twigs and pieces of wood are common throughout the deposit. A composite sample (see analysis 47, p. 34) was obtained by mixing peat from the different test holes. According to the analysis, it would not make good fuel.

Locality 4.-A deposit near Mystic Pond, about a mile west of Methuen and 3 miles northwest of Lawrence

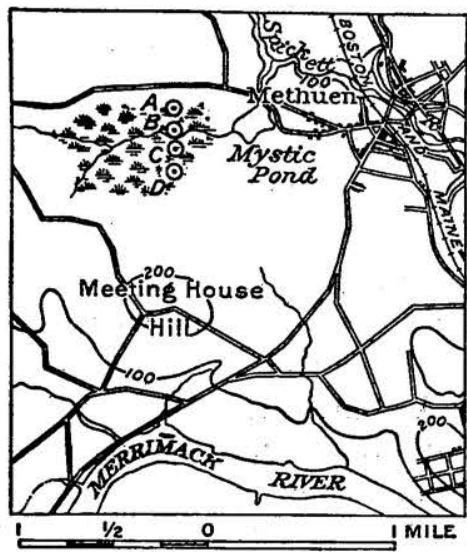

FIGURE 24. $\rightarrow$ Map of peat deposit near. Mystic Pond, Essex County, Mass., showing approximate position of test borings for peat. (see fig. 24), is about 200 acres in area and 6 feet in average depth. It should yield approximately 240,000 short tons of air-dried peat. The surface of the deposit is overgrown by grasses, cat-tails, reeds, and sedges and willow, alder, and poplar trees. The peat is of the built-up type. A series of test holes gave the following results:

Logs of test borings in deposit near Mystic Pond, west of Methuen, Mass.

Hole A:

Feet.

Dark-brown impure peat and black muck $\frac{1}{2}$

Brown fibrous peat $2 \frac{1}{2}$

Brown well-decomposed plastic peat

Holes B and C: Peat similar in character and depth to that in hole A.

Hole D : Peat similar in character to that in hole A $91065^{\circ}-22-12$ 
The black muck on the surface is ascribed to the ash resulting from the destruction several years ago by fire of the upper layer of the peat. No sample of this peat was analyzed. The deposit could be readily cleared and drained, and the peat might be used locally for fuel or fertilizer.

\section{MIDDLESEX OOUNTY.}

Locality 5.-About $1 \frac{1}{2}$ miles north of Littleton station and 1 mile southeast of Spectacle Pond (see fig. 25) there is a typical sphagnum. heath bog 100 acres in area. The vegetation consists chiefly of heath shrubs, sphagnum moss, and tamarack trees. The bottom of the deposit could not be reached with a 15-foot sounding rod. It is estimated that this deposit would yield at least 300,000 short tons of airdried peat. A series of test holes gave the following results:

Logs of test borings in bog north of Littleton station, Mass.

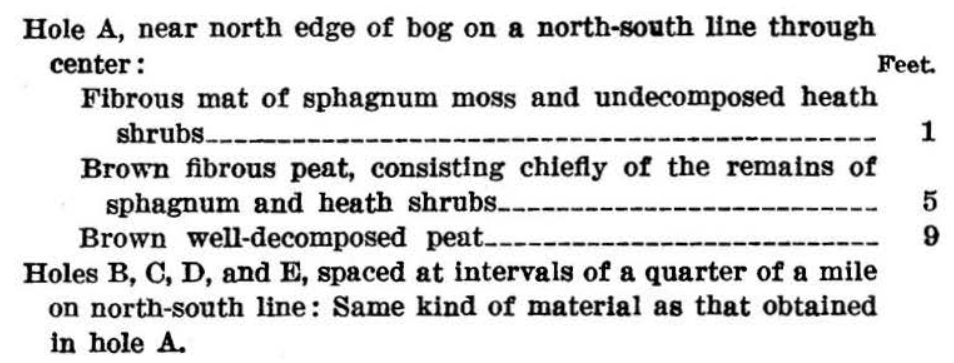

A composite sample (see analysis 26, p. 34) of the peat in this bog was obtained by mixing peat taken at intervals of 2 feet in depth from each of the test holes. The peat seems to be of excellent quality for fuel or fertilizer. The ash content (4.04 per cent) is unusually low. Large quantities of sphagnum moss, some of which is suitable for surgical use, could be obtained at this locality.

Locality 6.-A typical cat-tail marsh about a mile south of Littleton station, adjoining the south end of Mill Pond (see fig. 25), is about 100 acres in area, and the average depth of the deposit is 4 feet. A series of test holes gave the following results:

Logs of test borings in marsh south of Littleton station, Mass.

Hole F, about 25 yards west of the track of the Boston \& Maine Railroad, on an east-west line across the marsh a short distance south of its center:

Soft black muck

Feet.

Dark-brown well-decomposed plastic peat

Hole G, about 100 yards west of hole F: Same kind of material as that obtained in hole $\mathrm{F}$.

Hole $H$, about 100 yards west of hole $G$, near wagon road: Same kind of material as that obtained in hole $F$.

A composite sample (see analysis 27, p. 34 ) was obtained by mixing muck and peat taken at intervals of 1 foot in depth from each of the 
test holes. According to the analysis the deposit is too impure for use as fuel, but the unsatisfactory character of the sample is believed to be due to the upper layer of muck. If this marsh were well drained the peat could be used locally for fuel. It would be necessary, however, to strip off first the top layer of muck. Farmers in this locality are reported to have used peat from this deposit in earlier years. Part of the deposit has been burned since the last peat was cut. The deposit was probably thicker at that time. The muck at the surface may have been formed by ash resulting from the fire.

Locality \%.-A swamp adjoining Beaver Brook, about a quarter of a mile west of locality 6 (see fig. 25), is about 100 acres in area, is heavily forested, and contains black muck. No valuable peat was found.

Locality 8.-A deposit north of Duck and Knop ponds, about 2 miles southeast of Groton, was not examined but is reported to contain good peat.

Locality 9.-A peat meadow adjoining Meadow Brook, about 2 miles south of Lowell (see Pl. $\mathrm{XV}, B, \mathrm{p} .186$ ), which is skirted on the west and north by the Boston \& Maine Railroad, is shown on the map of the Lowell quadrangle, published by the United States Geological Survey. The deposit is about 400 acres in area and 4 feet in average thickness and should yield approximately 240,000 short tons of air-dried peat. The living vegetation consists chiefly of grasses and sedges. The peat is firm and well decomposed and is covered by black muck from 6 to 12 inches thick.

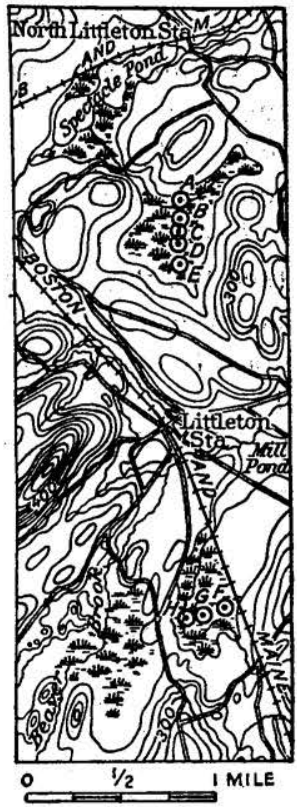

FigURE 25.-Map of peat deposits near Littleton and North Littleton stations, Middlesex County, Mass., showing approximate position of test borings for peat. The muck may have been formed either by the concentration of the ash in the peat by fire or by the silt and sand washed in by adjacent streams. A series of test borings gave the following results:

Logs of test borings in peat deposits on Meadow Brook, 2 miles south of Lowell, Mas8.

Hole A, about 25 yards east of railroad track, near west edge of meadow :

Feet.

Black muck

Brown firm, well-decomposed peat 
Hole B, about 100 yards east of hole A: Muck and peat similar in depth and texture to that in hole $\mathbf{A}$.

Hole C, about 100 yards east of hole B: Muck and peat similar in texture to that in hole A-

Hole $D$, about 100 yards east of hole $C$ : Muck and peat similar in depth and texture to that in hole $A$.

Hole $\mathrm{E}$, about 100 yards east of hole $\mathrm{D}$ : Muck and peat similar in depth and texture to that in hole $A$.

A composite sample (see analysis 29, p. 34 ) was obtained by mixing muck and peat taken at intervals of 1 foot in depth from each hole. By removing the overlying muck the peat in this deposit could be used locally as fuel.

Locality 10.-Tophet "Swamp," about a mile north of Carlisle (see fig. 26), is about 450 acres in area, and the deposit is 4 feet in average thickness. It is overgrown by plants of many species, notably sphagnum, grasses, sedges, the cranberry, shrubs, and trees. The peat, which is dark brown, firm, and well decomposed, is overlain by black muck of recent origin from 6 to 10 inches thick. A series of test borings gave the following results:

Logs of test borings in Tophet Swamp, north of Carlisle, Mass.

Hole A, near north edge of deposit at end of north-south line through center:

Black muck _-

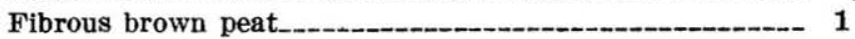

Brown well-decomposed peat, consisting of the remains of grasses and sedges

Hole B, about 100 yards south of hole A: Same kind of material as that obtained in hole $\mathrm{A}$.

Hole C, about 100 yards south of hole B : Muck and peat similar in texture to that obtained in hole A._._._._-_._._.

Hole D, about 100 yards south of hole C: Muck and peat similar in texture to that obtained in hole A_-_____-_-_ $3 \frac{1}{2}$

A composite sample (see analysis 30, p. 34 ) was obtained by mixing muck and peat taken at intervals of 1 foot in depth. The deposit seems to contain peat of excellent quality for local use as fuel or fertilizer. Some sphagnum moss suitable for surgical use occurs in the open parts of the area.

Locality 11.-A bog about $1 \frac{1}{2}$ miles south of South Chelmsford and 1 mile west of Tophet "Swamp" (see fig. 26) has been partly cleared at the north end and is being used for cranberry culture. The dominant vegetation formerly was sphagnum moss, heath shrubs, and tamarack trees. The area of the bog is about 300 acres, 30 or 40 acres of which is cultivated. The average thickness of the peat in the cranberry bog is more than 9 feet, but in the southern part it is only 5 feet. If the total area of workable peat is 200 acres and the average depth is 6 feet the bog should yield approximately 240,000 short tons 
of air-dried peat. Several test holes were put down at points along a line beginning at the north end of the cranberry bog and extending southward. The peat is fibrous near the surface but well-decomposed, soft, and plastic below. Analysis 31 (p. 34) shows the quality of the deep peat from the cranberry bog. The ash content is low and the fuel value is unusually high. The peat in this part of the deposit is of excellent quality for fuel. Analysis 32 (p. 34) shows the quality of the peat in the sphagnum-heath zone south of the cranberry bog. This peat is inferior in quality to that found to the north.

Locality 18.-A peat meadow adjoining Beaver Brook, about half a mile southeast of Forge Village,

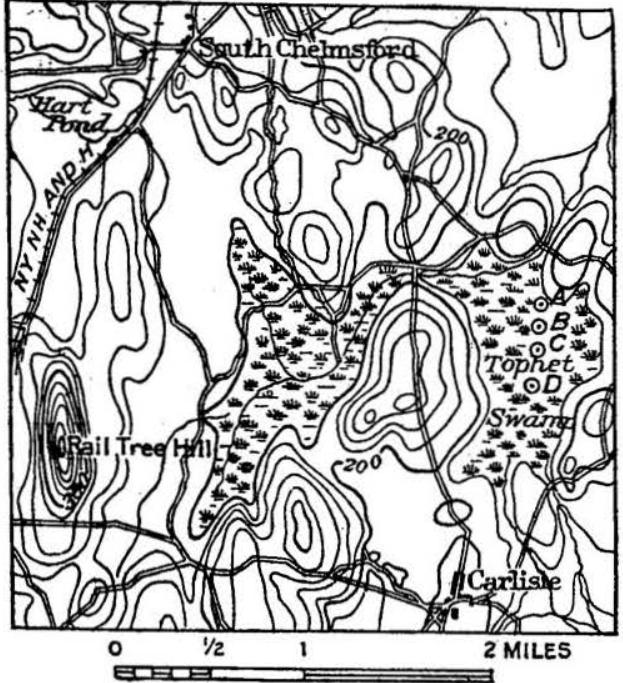

Figurw 26.-Map of Tophet Swamp and adjoining bog, Middlesex County, Mass., showing approximate position of test borings for peat. contains a deposit about 125 acres in area. The peat was not sampled but is reported to be about 6 feet thick and of good quality.

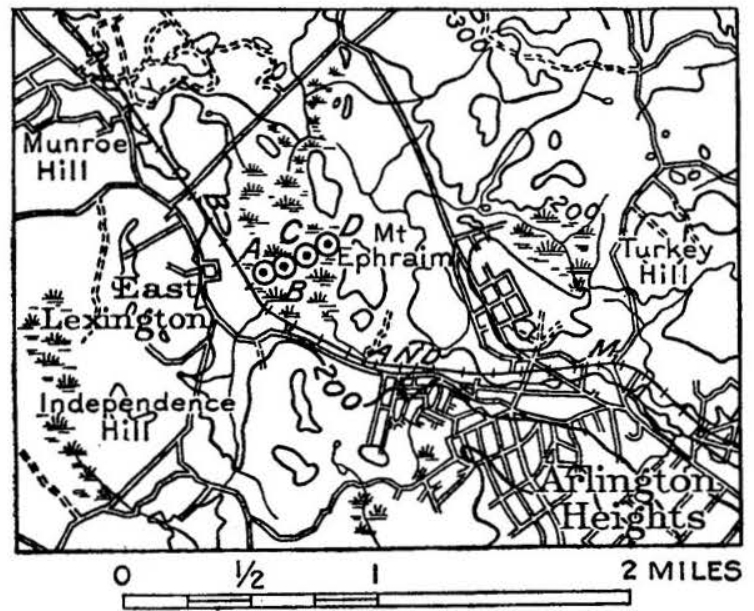

Frgore 27.-Map of deposit near Fast Lexington, Middlesex County, Mass., showing approximate position of test borings for peat.

Locality 13.-A deposit near the railroad station at East Lexington, a suburb of Boston (see fig. 27), is about 100 acres in area and 13 
feet in average thickness and should yield approximately 260,000 short tons of air-dried muck. A series of test borings gave the following results :

Logs of test borings in muck deposit near East Lexington, Mass.

Hole A, near west edge of deposit, about 50 yards northeast of old peat plant:

Brown well-decomposed soft, plastic muck

Greenish soft pond muck

Feet.

ole $B$, about 100 yards northeast of hole $A$, on line through

center of deposit:

Brown well-decomposed soft, plastic muck

Greenish soft pond muck

Hole C, about 100 yards northeast of hole B :

Brown well-decomposed soft, plastic muck

Greenish soft pond muck

Hole D, about 100 yards northeast of hole C:

Dark-brown well-decomposed soft, plastic muck _._._-_...- 3

Light-brown fine-grained soft pond muck-_-_--_-_-_-_-- 4

Separate representative samples were taken of the upper and lower portions of the deposit, each of which was obtained by mixing

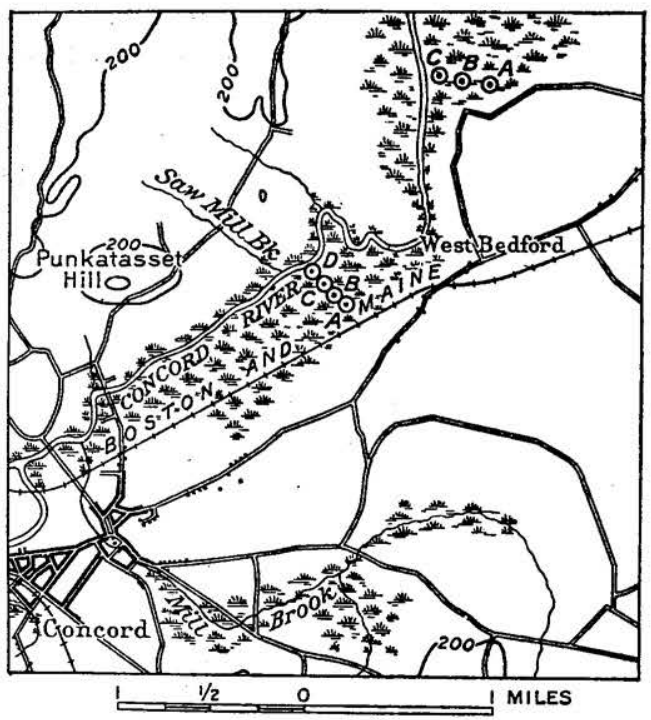

Fraure 28.-Map of marshes adjoining Concord River, Middlesex County, Mass., showing approximate positions of test borings for peat. muck taken at intervals of 2 feet in depth from each hole. Analysis 34 (p. 34) shows the quality of the upper 8 to 10 feet of brown plastic muck and analysis $34 \mathrm{a}$ that of the lower 10 feet of green pond muck. This deposit is advantageously located and contains some muck that is high in nitrogen. The ash content is too high for the material to be used as fuel. A large plant for the manufacture of fertilizer was erected on this bog several years ago by the Boston Humus Co., of East Lexington, . Mass., and was successfully operated until recently. Although the plant is not now operated, it is
still in good condition and could be reopened at moderate expense. This deposit seems to be one of the best in New England for the production of peat fertilizer. It is not as large as some others in Massachusetts, but it contains good raw material and is easily accessible. 
Locality 14.-A muck meadow about a mile east of Bedford, which is in part being used for the cultivation of hay, was examined, but no peat was found. The meadow is covered with a shallow accumulation of black muck, which appears to be more valuable for enriching the soil of the locality than for the production of fertilizer.

Locality 15.-A marsh adjoining Concord River about a mile north of West Bedford (see fig. 28) extends southwestward along Concord and Sudbury rivers from 10 to 12 miles, but only the part north of West Bedford, consisting of approximately 500 acres, is included in this locality. The average thickness of this part of the deposit is 4 feet, and it should therefore yield about 400,000 short tons of air-dried muck and peat. The living vegetation consists chiefly of the cranberry, grasses, sedges, ferns, and small shrubs. Test holes showed material of character and thickness as follows:

Logs of test borings in marsh on Concord River north of West Bedford, Mass.

Hole A :

Feet.

Black muck

$\frac{1}{2}$

Brown fibrous peat___________ 1

Hole. B :

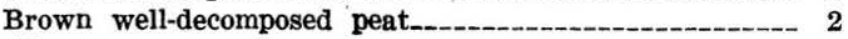

Black muck -

Brown fibrous peat_-_-___-_-_ $1 \frac{1}{2}$

Hole C:

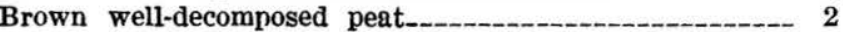

Black muck --_-_-_- $\frac{1}{2}$

Brown fibrous peat_-_-_-_-_._- $1 \frac{1}{2}$

Brown well-decomposed peat_-_._-____

A composite sample (analysis 35, p. 34 ) was obtained by mixing muck and peat taken at intervals of 1 foot in depth from each hole.

Locality 16.-A marsh along the south side of Concord River, about 1 mile northeast of Concord (see fig. 28), contains a deposit about 400 acres in area and 6 feet in average thickness, which should yield 480,000 short tons of air-dried muck and peat. A series of test borings gave the following results:

Logs of test borings in marsh on Concord River northeast of Concord, Mass.

Hole A, north of rallroad track at south edge of swamp, sixtenths of a mile southwest of West Bedford: Feet.

Black muck -.._-_-

Brown fibrous to well-decomposed muck

Hole B, about 100 yards northwest of hole A :

Black muck

Brown fibrous to well-decomposed muck-_-_-_._-_-_-_-_- $5 \frac{1}{2}$

Hole C, about 100 yards northwest of hole B :

Black muck

Brown fibrous to well-decomposed, impure peat_-_-_-_-_.- $5 \frac{1}{2}$

Hole D, near Concord River, abcut 100 yards northwest of hole $\mathbf{C}$ :

Black muck -...

Brown flbrous to well-decomposed, impure peat._...-_...- $5 \frac{1}{2}$ 
A composite specimen (see analysis 36, p. 34 ) was obtained by mixing samples from the four holes. The material in this deposit is too high in ash and too low in nitrogen for use as fuel or fertilizer.

Locality 17.-A deposit adjoining Sudbury River, about half a mile southwest of Concord. No peat was found at this locality, but about 50 acres near the river is covered with a shallow deposit of black muck.

Locality 18.-A marsh along Assabet River, north of Concord Junction, contains a deposit similar to that at locality 17.

Locality 19.-In a marsh adjoining Fort Pond Brook, about a mile northwest of West Acton, no valuable peat was found. A sample (see analysis 37, p. 34) was taken from a test hole near the center. The deposit appears to contain muck that is of no value for fuel and that is too low in nitrogen for fertilizer. The living vegetation consists chiefly of reeds, grasses, sedges, ferns, small shrubs, and the cranberry.

Locality 20.-A marsh about a mile south of West Acton, which is several hundred acres in area, contains no valuable peat. The living vegetation consists chiefly of grasses, sedges, cat-tails, the cranberry, and small ferns.

A composite sample of the deposit (see analysis 38, p. 34 ) was obtained by mixing material from several test holes. The deposit contains muck which can not be used for fuel.

Locality 21.-A marsh surrounding Heird Pond and adjoining Wash Brook and Sudbury River, which is several square miles in area, contains little valuable peat. Analysis 39 (p. 34) shows the character of a composite sample and indicates that the deposit consists largely of muck that is of no value for fuel or fertilizer.

Locality 22.-A marsh adjoining Sudbury River and surrounding Baldwin Pond, about $1 \frac{1}{2}$ miles northwest of Wayland, lies directly north of loeality 21 and is of the same type. Analysis 40 (p. 34) shows the character of a composite sample and indicates that the deposit consists largely of muck that is of no value for fuel.

Locality 23.-A swamp south of Shakum Pond and west of the Milford branch of the Boston \& Albany Railroad contains a deposit about 50 acres in area and 4 feet in average thickness, which should yield 40,000 short tons of air-dried peat. The dominant living vegetation consists of maple, poplar, and elm trees. Two test holes gave the following results: Logs of test borings in swamp south of Shakum Pond, near South Framing-
ham, Mas8.

Hole A, in center of hay meadow west of car track:

Black muck

Feet.

Brown firm well-decomposed, plastic peat.

Black muck. 
Hole B, about 200 yards east of hole A:

Black muck

Dark-brown fibrous, woody peat.

Fléet.

$\therefore \frac{1}{2}$

4

Analysis 41 (p. 34) shows the quality of the peat from hole A. The deposit is probably too shallow to justify the erection of a plant but could well be used for the production of cut peat.

Locality 24.-A small, deep filled-basin deposit near Bow Brook a mile southwest of Shirley contains about 3 acres of valuable peat 8 feet in average depth. A small peat plant was erected several years ggo near the west edge, and an attempt was made to produce machinepeat fuel. The deposit, however, was not of sufficient size to justify such a project, and the attempt was soon abandoned. A series of test borings gave the following results:

Logs of test borings in deposit near Bow Brook, 1 mile west of Shirley, Mass.

Hole A, near east edge of deposit at site of old peat plant: Feet.

Brown tbrous peat.-_-_._- 1

Brown partly to thoroughly decomposed peat_________- 13

Hole B, near center: Brown partly to thoroughly decomposed peat.-_-_- 10

Hole $\mathrm{C}$, near west elge: Brown partly to thoroughly decomposed peat_-_-_._- 8

A composite sample (see analysis 44 , p. 34 ) was obtained by mixing peat taken at intervals of 2 feet in depth. The peat appears to be of fair quality for fuel or fertilizer. Although the deposit is too small to warrant the erection of a plant, enough peat could be cut to supply the farmers of the vicinity with fuel for several years.

Locality 25.-Part of the meadow that adjoins Sudbury River near the confluence of Pantry and Cold brooks contains a deposit several bundred acres in area, which should yield 480,000 short tons of airdried peat. The dominant vegetation consists of sedges, sphagnum, ind the cranberry. A test boring put down near the mouth of Cold Brook gave the following result:

Log of test boring in meadow on Sudbury River near the mouth of Cold Brook, Mass.

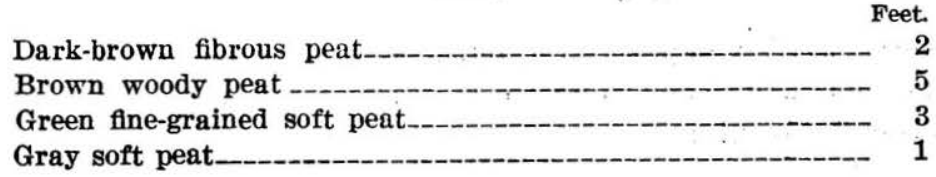

Locality 26.-A meadow that surrounds Fair Haven Pond on Sudbury River, about 3 miles east of North Sudbury, contains a deposit bout 150 acres in area and 7 feet in average thickness, which should pield approximately 210,000 short tons of air-dried peat. The vegetation consists chiefly of willow trees, shrubs, ferns, and grasses. Test 
holes put down near the north and south ends of Fair Haven Pond gave the following results:

Logs of test borings in meadow surrounding Fair Haven Pond, 8 miles east of North Sudbury, Mass.

Hole A, south of Fair Haven Pond, on east side of Sudbury River :

Feet.

Dark-brown fibrous to macerated woody peat________- 1

Reddish-brown fibrous to macerated peat_-_______- 4

Dark-brown to reddish-brown somewhat woody peat__.__- 1

Dark-brown well-decomposed somewhat silty peat_______- 2

Green fine-grained peat

Hole B, north end of Fair Haven Pond near east bank of Sudbury River :

Black to gray peaty soil

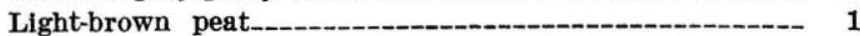

Brown well-decomposed peat with woody components__._- 2

Brown to green well-decomposed peat__-___- 2

Gray well-decomposed peat_-_ 1

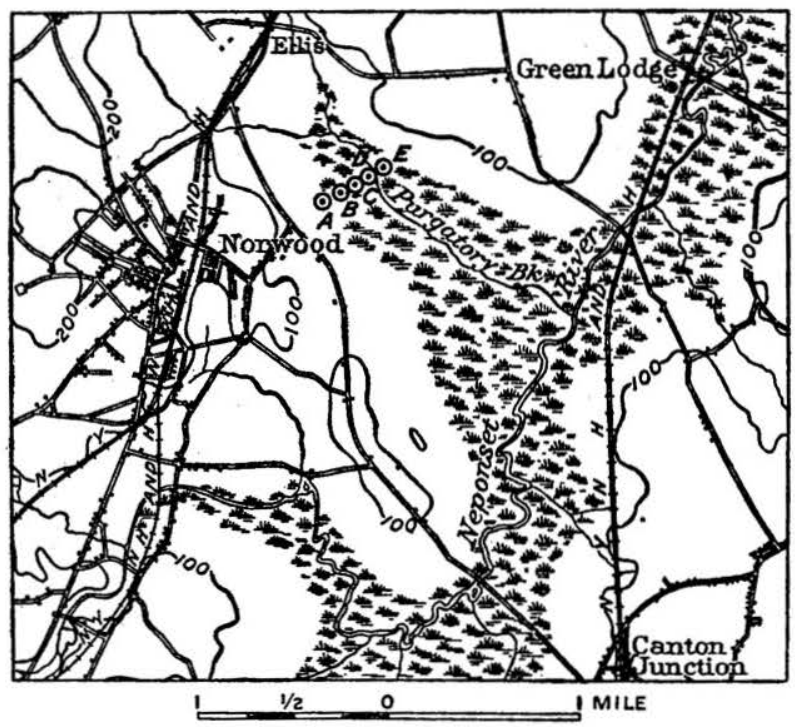

Fiqurn 29.-Map of peat bog near Norwood, Norfolk County, Mass, showing approximate pasition of test borings for peat.

Locality 2\%-A deposit in a kettle hole in glacial drift adjoining the Wheeler farm, near Concord, was tested by a boring, which gave the following result:

Log of test boring near Wheeler farm, Concord, Mass.

Reddish-brown woody peat

Dark-brown woody peat_._. 3

Red to brown coarsely fibrous peat_____ 5

Coarsely fibrous, matted, leafy peat________ 4

Fine-grained peat, composed chiefly of debris of aquatic plants_ $\quad 3$ 
MORTOLK OOUNTY.

Locality 28.-A large meadow bog that adjoins Purgatory Brook about 1 mile east of Norwood (see fig. 29) contains a deposit about 600 acres in area and 10 feet in average thickness, which should yield approximately 1,200,000 short tons of air-dried peat. It extends southeastward from Norwood to the mouth of the brook, where it connects with the marsh on Neponset River. Peat fuel and fertilizer have been produced at this locality since 1918. The deposit is of the built-up type, and the dominant vegetation consists of grasses, sedges, ferns, sphagnum moss, heath shrubs, and the cranberry. The peat is relatively homogeneous and varies from dark brown to yellowish brown. Test holes put down in the deposit gave the following results:

\section{Logs of test borings in bog on Purgatory Brook, about 1 mile east of Nor- wood, Mass.}

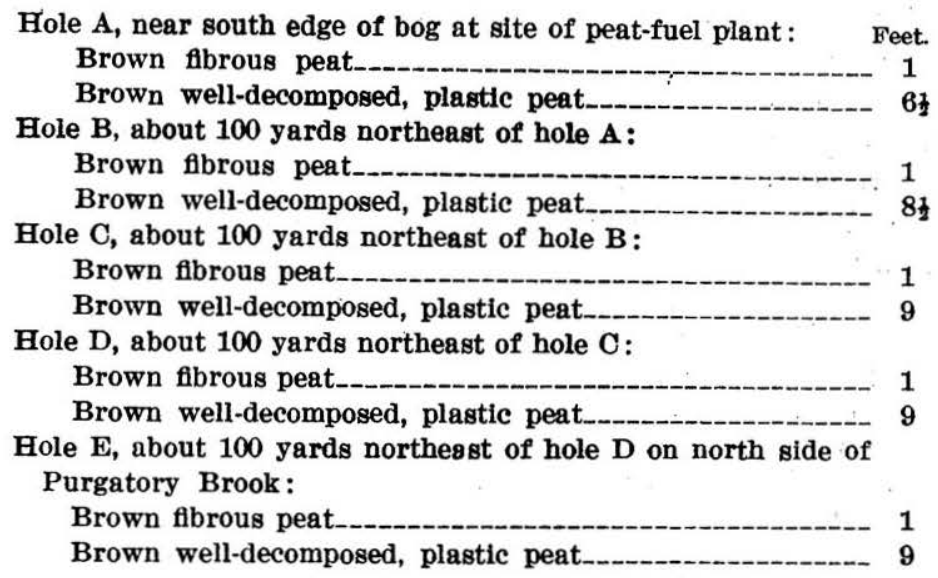

A composite sample (see analysis 42, p. 34 ) was obtained by mixing peat taken at intervals of 2 feet in depth from each hole. The ash content is low, and the fuel value and nitrogen content are well above the average. The peat therefore seems to be of good quality for fuel or fertilizer. Analysis 42a (p. 34) shows the quality of a sample obtained from a stock pile of peat excavated for the production of fuel. Analysis 42b (p. 34) shows the composition of a sample of finished peat fuel manufactured in an experimental machine.

A part of this deposit has been leased by Peat Coal (Ltd.), of Boston, Mass., and a fuel plant was being erected when the peat was sampled. In the process selected the peat is taken from the bog by a screw excavator mounted on a dredge and transported by overhead conveyors or scows to the plant, which has a capacity of 300 to 500 short tons of peat a day. At the plant the peat is fed to a macerator, which thoroughly disintegrates and works it into a plastic mass. 
About 50 per cent of the moisture is then removed by artificial drying and 30 per cent by air-drying. The company proposes to market the peat in the form of cylindrical briquets.

At the time the plant was visited good peat fuel was being produced by a small, simple machine and stored for use in the operation of the larger plant then under construction. Parts of the bog had been subleased to local residents, who were cutting peat for home use. This hand-cut peat. was of excellent quality and indicates the possibilities of the numerous small bogs scattered throughout the State. This bog is near Boston, and little difficulty should be met in marketing fuel obtained from it.

Locality 29.-A small bog about midway between Walpole and East Walpole contains a deposit about 5 acres in area and 12 feet in average thickness, which should yield about 12,000 short tons of air-dried peat. It occupies a kettle hole and is of the typical filled-basin type. The living vegetation consists chiefly of heath shrubs, sphagnum moss, the cranberry, cotton grass, and sedges. The peat is dark brown to reddish brown, well decomposed, plastic, and of homogeneous texture. It is free from roots, logs, and pieces of wood and is well adapted to the manufacture of machine-peat fuel. Three test holes sunk on a line through the center of the bog gave the following results:

Logs of test borings in bog between Walpole and East Walpole, Mass.

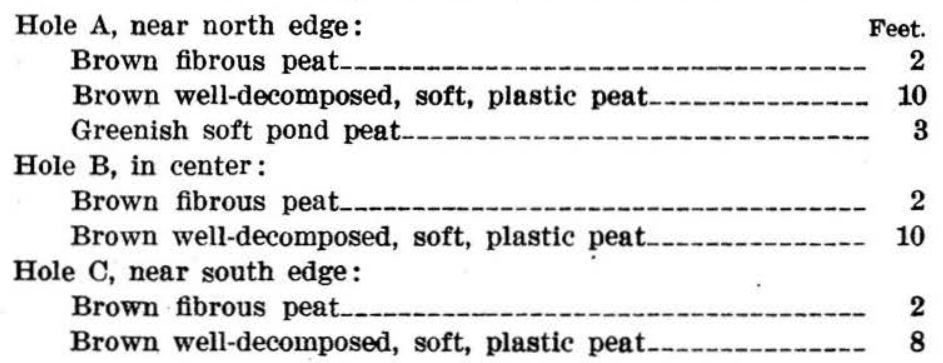

A composite sample (analysis 43, p. 34 ) was obtained by mixing peat taken at intervals of 2 feet in depth from each hole. The deposit contains peat of good quality for fuel.

Several years ago Philip Allen, of Walpole, Mass., the owner of the bog, undertook the production of machine-peat fuel, but the project was abandoned. Although the peat is suitable for fuel, the deposit is too small for commercial use. However, a substantial quantity of peat could be cut and used by local residents.

Locality 30.-Adjoining the western shore of Charles River southwest of West Roxbury there is a typical river marsh that has been formed in a river valley subject to frequent overflow. (See fig. 30.) The living vegetation consists chiefly of grasses and sedges. A series 
of test holes was put down, but no valuable peat was found. Analyses 46 and 46a (p. 34) show the composition of composite samples obtained from these holes and indicate that the deposit consists largely. of muck.

Locality 31.-A marsh that adjoins the western shore of Charles River north of Dedham Island and east of Needham (see fig. 30) contains a deposit similar in physical and chemical characteristics to that in Locality 32.

Locality 32.-An extensive marsh adjoins both shores of Neponset River and extends from Ashcroft to Canton Junction. The living regetation consists chiefly of grasses, sedges, and shrubs. This marsh is several square miles in area and contains a deposit of muck and impure peat from 1 to 5 feet thick. Dachnowski ${ }^{51}$ thus describes the area:

With the exception of the part on Purgatory Brook, the most striking feature of this deposit is an extensive surface layer of diatomaceous siliceous material adjacent to the river. The deposit ranges in thickness from 8 inches to 4 feet and is derived almost wholly from microscopic plants, mainly diatoms, which float freely in the quiet waters of ponds and ilvers. Throughout nearly the length of the river this layer rests on older beds of peaty material that range in thickness from 1 to 5 feet. Most of this peaty material is well decomposed and fairly compact and generally rests on micareous sand. The plant remains are derived in large part from transported organic material and from herbaceous und shrubby vegetation.

Soundings in the part of the narsh that lies on Purgatory Brook indicate a bed of diatonaceous earth as a bottom

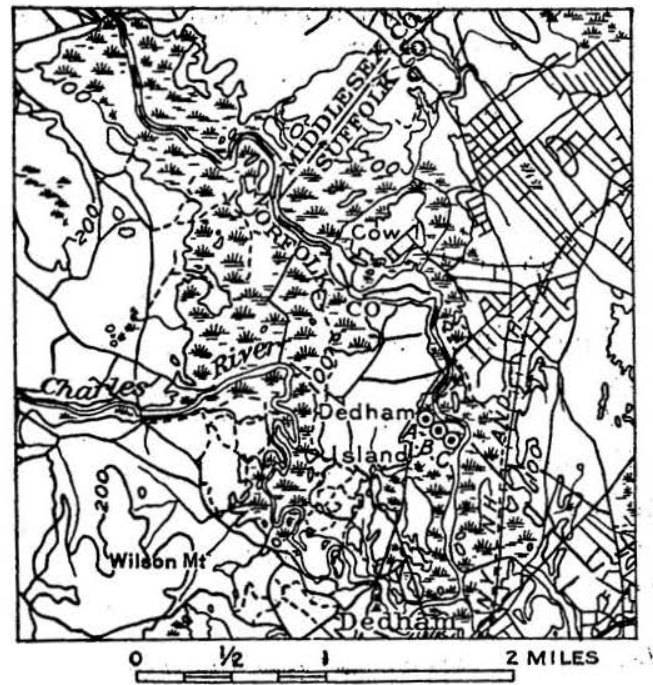

Figure 30.-Map of marsh adjoining Charles River west of Boston, Mass., showing approximate position of test borings for peat $(\mathrm{A}, \mathrm{B}, \mathrm{C})$.

ver in the deeper depressions. The overlying materials are types of fibrous und wicker-like peats. They are plant remains derived from vegetation units ihich appear to have succeeded each other in the formation of this deposit in sponse to changes in the ground-water table.

\section{PIYMOUTH COUNTY.}

Locality 33.-Great Cedar Swamp, about half a mile south of South Hanson, adjoins Monponset and Stump ponds on the north. Its location and area are shown on the map of the Abington quad-

\footnotetext{
Dachnowski, Alfred, unpublished notes.
} 
rangle, published by the United States Geological Survey. Forest trees and underbrush which would be difficult to remove are the principal forms of plant life, and the peat therefore contains many roots, logs, and pieces of wood.

Locality 34-A cranberry bog near Halifax is shown on the maps of the Abington and Middleboro quadrangles, published by the United States Geological Survey. A series of test holes in this bog gave the following results : ${ }^{52}$

Generalized log of test borings in cranberry bog near Halifax, Mass.

Feet.

Red to dark-brown fine-grained to granular peat, containing woody matter

Dark-brown well-decomposed to fine-grained peat, containing charred woody matter

Red to dark-brown peat, contrining leaf-mold and pine needles_ 1

Dark-brown to reddish-brown partly disintegrated, somewhat fibrous peat, containing roots, flbers, and woody matter

Reddish-brown fine-grained to flbrous peat, containing charred woody matter

Reddish-brown fibrous to matted peat, containing twigs and remains of heath shrubs.

Brown well-decomposed to partly fibrous peat, somewhat silty, containing woody matter

Yellowish-brown well-decomposed peat, containing twigs, pieces

of wood, and sand

Grayish coarse to fine, well-decomposed, stratified silty peat_-_- $1 \frac{1}{2}$

The living vegetation of this bog consists chiefly of sphagnum, the cranberry, and red maple and cedar trees.

Locality 35.-Great Cedar Swamp, about 4 miles northeast of Middleboro, is shown on the map of the Middleboro quadrangle, published by the United States Geological Survey. The dominant vegetation consists of red maple, hemlock, birch, cedar, and white pine trees. A series of test holes gave the following results: ${ }^{52}$

Logs of test borings in Great Cedar Swamp, 4 miles northeast of Middleboro, Mass.

Hole A, south end of swamp near west border :

Feet.

Dark-brown to reddish-brown fibrous peat, containing roots of living plants, twigs, and other vegetable matter

Black fibrous peat

1

Black to red fibrous to well-decomposed peat

Hole $B$, east of hole $A$, about halfway across swamp : Darkbrown to red partly decomposed to flbrous peat, containing twigs and other woody components

sachnowski, Alfred, unpublished notes. 
Hole $\mathbf{C}$, east of hole $\mathbf{B}$, about halfway between middle and east edge of swamp:

Feet.

Dark-brown disintegrated to fibrous peat, containing twigs and woody components

1

Black fine-grained peat

Black well-decomposed fine-grained peat

Hole D, east of hole C, south end of swamp near east margin :

Reddish-brown to black fine-grained peat________-_-_ 2

Black well-disintegrated peat, somewhat silty

Hole E, near center of swamp :

Dark-brown flbrous to disintegrated peat, containing twigs and woody components

Reddish-brown fibrous to disintegrated, plastic peat._._.-

Dark-brown well-decomposed peat

Red to dark-brown fine-grained peat, containing woody matter

Dark-brown well-decomposed peat.

Hole $\mathrm{F}$, midway between hole $\mathrm{E}$ and east edge of swamp: Same character of material as that obtained in hole E.

Hole $G$, east of hole $F$, near east margin of swamp: Darkbrown flbrous to disintegrated peat, containing woody matter

\section{WOROESTER COUNTY.}

Locality 36.-Great Swamp, between Dunn Brook and East Brookfeld River (see fig. 31), is about 350 acres in area and contains a deposit

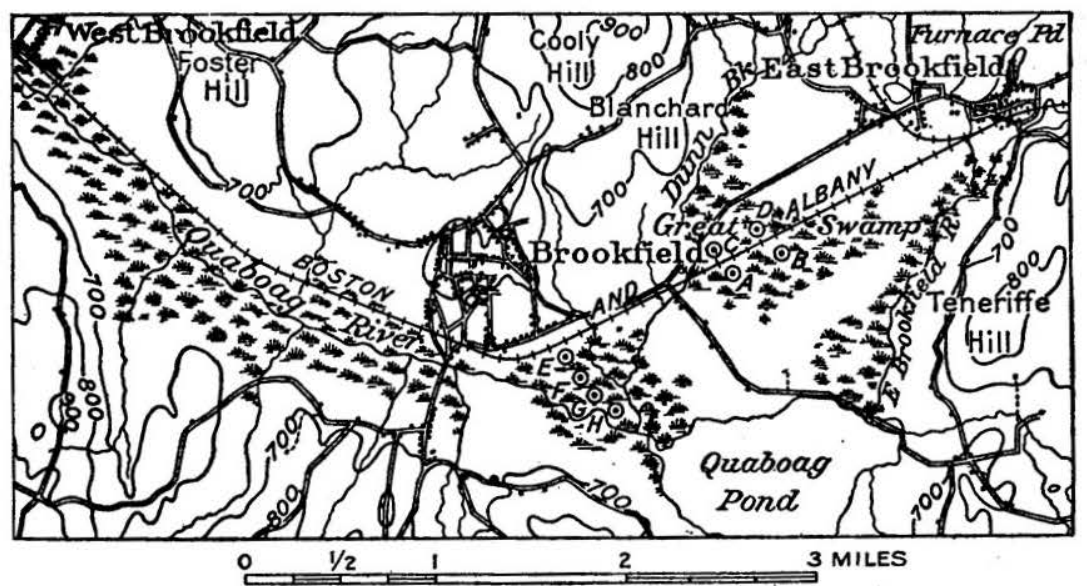

houre 31.-Map of Great Swamp and marsh adjoining Quaboag River, Worcester County, Mass., showing approximate position of test borings for peat.

of peat $3 \frac{1}{2}$ feet in average thickness, which should yield approximately 245,000 short tons of air-dried peat. It is thickly wooded with red maple, elm, alder, and other broad-leaved trees. A series of test oles gave the following results: 
Logs of test borings in Areat Swamp, between Dunn Brook and East Brookfeld River, Mass.

Feet.

Hole A, south of railroad track near west edge of swamp: Black impure peat and muck

Hole B, south of track near center of swamp: Black sandy peat

Hole $\mathrm{C}$, between track and wagon road near western edge of swamp: Brown well-decomposed, plastic peat

Hole D, midway between track and wagon road, near center of swamp: Brown well-decomposed, plastic peat_

A composite sample (see analysis 62 , p. 34 ) was obtained by mixing peat taken at intervals of 1 foot in depth from holes $C$ and $D$. The peat appears to be of good quality for fuel or fertilizer. However, as the deposit would be difficult to drain and clear and the thickness is variable, it is not a promising source of peat for commercial use.

Locality 37.-A marsh extends westward along Quaboag River from Quaboag Pond to West Brookfield, a distance of about 4 miles (see fig. 31), but only the part near the pond contains good peat. The living vegetation consists chiefly of sedges, grasses, and cattails, and the peat, which is made up chiefly of the remains of these plants, is well disintegrated. The peat deposit is about 350 acres in area and 7 feet in average thickness and should yield approximately 490,000 short tons of air-dried peat. A series of test holes put down at points along a line extending southeastward across the center of the depasit gave the following results:

Logs of test borings in marsh on Quaboag River, Mass.

Hole A, near north edge:

Black muck

Feet.

Brown well-decomposed, plastic peat

Hole B, about 200 yards southeast of hole A :

Black muck

Brown well-decomposed, plastic peat, composed of the remains of grasses and sedges.

Greenish soft pond peat_.

Hole C, about 200 yards southeast of hole B :

Black muck

Brown well-decomposed, plastic peat, composed of the remains of grasses and sedges

Hole $D$, about 200 yards southenst of hole $\mathrm{C}$ :

Black muck

Brown plastic peat, composed of the remains of grasses and sedges

Greenish soft pond peat

A composite sample (see analysis 63 , p. 34 ) was obtained by mixing peat taken at intervals of 2 feet in depth from each hole. The peat contains too much ash for use as fuel, but it may be of some value for agricultural purposes. 
Locality 38.-A marsh about a mile southwest of Harvard station, which is shown on the map of the Groton quadrangle, published by the United States Geological Survey, is about 640 acres in area. It is overgrown by cat-tails, sedges, reeds, small willows, and trees and shrubs. The deposit is of the built-up type and occupies the bottom land of Nashua River. The peat, which is firm and well decomposed, is overlain by 6 to 12 inches of black muck and is underlain by sand. The muck was probably formed as the result of a fire many years ago. A series of test borings gave the following results:

Logs of test borings in marsh on Nashua River, near Harvard station, Mass.

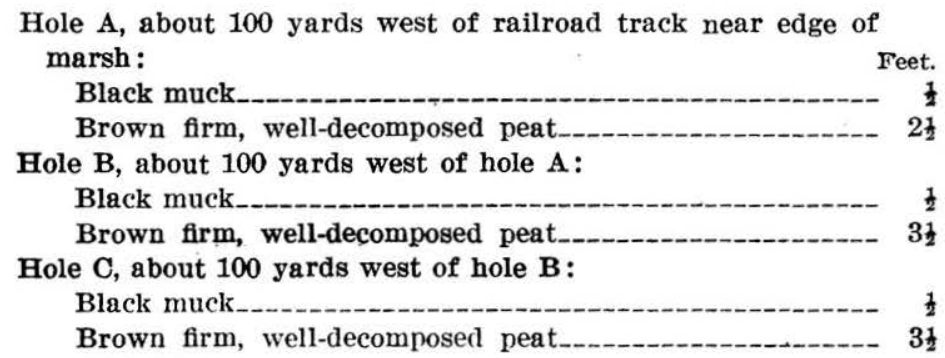

A composite sample (see analysis 28, p. 34 ) was obtained by mixing peat and muck taken at intervals of 1 foot in depth from each hole. According to the analysis the deposit as a whole is too high in ash for use as fuel, but the high ash is probably due to the overlying muck. The deposit could probably be most advantageously used for agricultural purposes.

\section{CONNECTICUT.}

\section{GENERAL FEATURES.}

Although some peat is found in western Connecticut, the largest and most valuable deposits occur in the northern and eastern parts of the State. Spruce and cedar swamps, which are the predominant types of undrained land, occur at the following places: Botsford, Neversink Pond, and New Fairfield, Fairfield County ; Berlin, Plantsville, Southington, South Windsor, and West Suffield, Hartford County; Benedict Pond, Chapinville, and Norfolk, Litchfield County; and Stonington, New London County. There are also many salt marshes along the coast, as well as numerous maple, ash, and elm swamps in central and eastern Connecticut. Marl underlies peat in some of the northern Connecticut deposits, notably in the tamarack swamps surrounding Twin Lakes, Litchfield County. A bed of marl 25 feet deep is reported to underlie West Twin Lake. The types of peat deposits and processes of formation in Connecticut are essentially the same as those in the other New England States. 
Although Connecticut has numerous and widely distributed peat deposits they are relatively small, and the total quantity of peat that could be obtained from them is much less than the quantity available in the deposits either of Vermont or Massachusetts. The deposits in Connecticut that have been examined are capable of yielding about $1,000,000$ short tons of air-dried peat. However, several of the large deposits and the numerous scattered small bogs have not been tested. Those deposits would probably yield at least 1,000,000 tons, and the total peat resources of the State would therefore amount to about $2,000,000$ short tons.

The following localities were tested for peat in the progress of the field work upon which this report is based.

\section{FAIRFIELD COUNTY.}

Locality 1.-A swamp which is shown on the maps of the Danbury and Derby quadrangles, published by the United States Geological Survey, begins about a quarter of a mile south of Botsford and extends southward for about $1 \frac{1}{2}$ miles. The swamp is crossed by the track of the New York, New Haven \& Hartford Railroad, and much of the area is owned by the railroad. The deposit covers several hundred acres, but only about 100 acres contain good peat, the average thickness of which, as indicated by test holes, is about 4 feet. The deposit is estimated to be capable of yielding about 80,000 short tons of air-dried peat. The living vegetation consists chiefly of deciduous trees, notably red maple and elm, though a few scattered spruce trees as well as some ferns and sphagnum are also present. Some of the sphagnum is of the large leafy type, suitable for surgical use. Test holes were made in different parts of the swamp, but only a few of them yielded peat. The peat; which is of the forest type, is shallow and fibrous and contains considerable woody fiber near the top but is plastic and well decomposed at a depth of 2 or 3 feet. The best peat was found in the following holes:

Logs of test borings in swamp south of Botsford, Conn.

Hole A, near north edge, east of railroad track: Dark-brown
peat, woody near top, clayey and fine grained below
Hole B, beside railroad track, about one-fourth of a mile south
of hole A: Brown fibrous peat, containing leaves and twigs_--
Hole $\mathrm{C}$, beside railroad track, about one-fourth of a mile south
of hole B: Brown fibrous to woody peat------
Hole $\mathrm{D}$, at roadside in north central part: Brown well-decom-
posed peat, containing twigs, leaves, and small pieces of
wood

\section{Feet.} 4

A composite sample (see analysis 57, p. 19) from a tract of about 15 acres in the area near the north edge of the swamp was obtained by mixing peat taken at intervals of 1 foot in depth from cach hole. 
The peat in this tract appears to be of good quality for fertilizer, but its texture makes it unsuitable for the manufacture of fuel. Analysis 58 (p. 19) gives the composition of a sample taken about half a mile south of Botsford on the west side of the New York, New Haven \& Hartford Railroad track and indicates that the peat in this area is suitable for fuel or fertilizer.

\section{HARTFORD COUNTY.}

Locality 2.-A bog about 3 miles north of Cromwell, which is shown on the map of the Middletown quadrangle, published by the United States Geological Survey, is about 37 acres in area, but not all of this acreage contains workable peat. A small filled basin about 10 acres in area and 10 feet in average depth contains the best peat. The area should yield approximately 20,000 short tons of air-dried peat. The living vegetation of the 10-acre tract consists chiefly of grasses, sedges, shrubs, and sphagnum. In the surrounding woods red maple, .alder, ash, and elm predominate. Except in the upper 2 feet the peat is well decomposed, plastic, and of homogeneous texture.

Logs of test borings in bog north of Cromwell, Conn.

$\begin{array}{lr}\text { Hole A, near south edge: } & \text { Feet. } \\ \text { Brown fibrous peat-- } & 2 \\ \text { Brown well-decomposed, soft plastic peat, consisting chiefly } & \\ \quad \text { of the remains of grasses and sedges_- } & 4 \\ \text { Green soft clayey pond muck- } & 4 \\ \text { Hole B, near center: Peat and muck similar to that in hole A_- } & 12 \\ \text { Hole C, north edge: Peat and muck similar to that in hole A_ } & 10\end{array}$

A composite sample (see analysis 59, p. 19) was obtained by mixing peat and muck taken at intervals of 2 feet in depth from each hole. The material as a whole appears to be too high in ash for use as fuel. The nitrogen content is 1.32 per cent. It may be, howlever, that the upper 6 feet could be used for fuel, but care should ibe taken to avoid the lower layers of pond muck, which are very high in ash. The deposit has been leased by A. N. Pierson, of Cromwell, Conn., who has attempted to substitute powdered peat for coal for use in heating his greenhouses.

MIDDLESEX COUNTY.

Locality 3.-A small bog about one-quarter of a mile southeast of Hocality 2 , which is only 2 acres in area, contains a deposit of peat and muck about 3 feet deep. The living vegetation is made up largely of grasses, sedges, and sphagnum. The deposit consists of fibrous peat and muck containing much sand. It has been leased by A. N. Pierson, of Cromwell, Conn., who attempted to use the peat and muck for fuel, but the ash content was so high that the 
project was unsuccessful. Analysis 60 (p. 19) gives the composition of a specimen taken from Pierson's stock pile and shows that the ash content is 58.25 per cent and the nitrogen 1.48 per cent.

Locality 4.-A marsh near the confluence of Mattabesset and Connecticut rivers, about one-fourth of a mile north of the railroad station at Middletown, which is shown on the map of the Middletown quadrangle, published by the United States Geological Survey, contains a deposit about 600 acres in area that consists of only shallow muck. Locality 5.-Durham Meadows, which are shown on the map of the Guilford quadrangle, published by the United States Geological Sur-

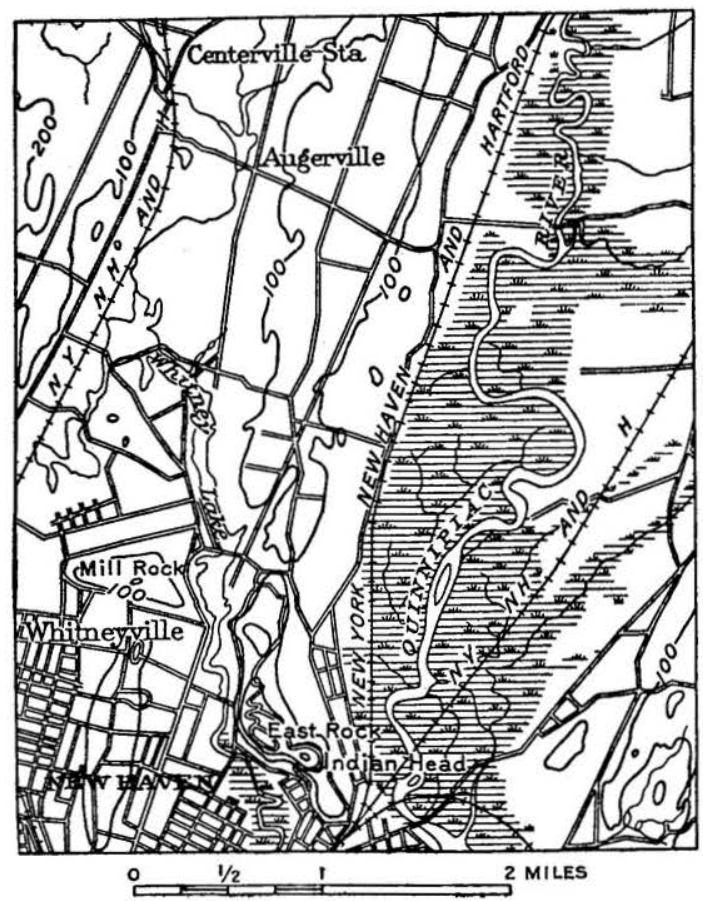

FivURe 32.-Map of salt marsh adjoining Quinnipiac River, New Haven County, Conn. vey, extend along both sides of Coginchaug River, about onefourth of a mile west of Durham. These meadows are about 1,000 acres in area but contain no peat of commercial importance.

\section{NEW HAVEN COUNTY.}

Locality -A salt marsh that adjoins Quinnipiac River between North Haven and New Haven (see fig. 32) is several hundred acres in area, but only the part near the Davis brickyard, about 4 miles north of New Haven, a tract of about 300 acres, contains high-grade peat. The thickness of the deposit ranges from 4 to 7 feet, and the average depth is about 5 feet. This tract should yield approximately 300,000 short tons of air-dried peat.

The deposit as a whole consists of 2 to 3 feet of salt-marsh peat underlain by 2 or 3 feet of fresh-water peat. (See Pl. XV, A.) The fresh-water peat is dark brown to black, well decomposed, and plastic and contains well-preserved remains of ferns, sedges, and grasses. The salt-marsh peat is fibrous and somewhat silty. The section illustrated in part by Plate XV, $A$, was exposed by excavations for clay. Several old stumps of trees 12 inches in diameter 


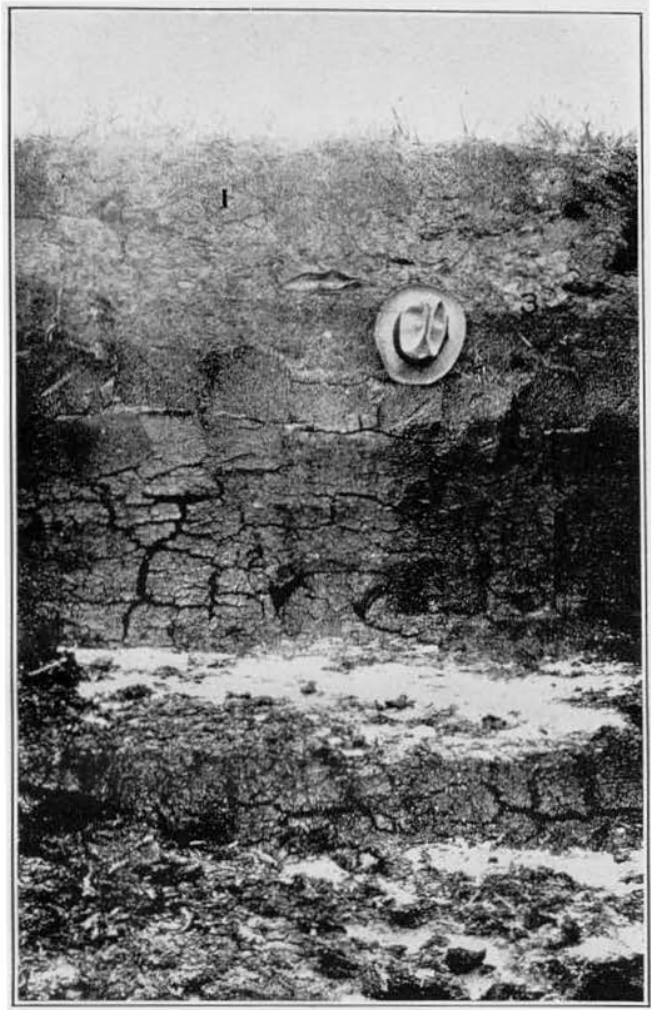

A. CROSS SECTION OF COMPOSITE PEAT BED NEAR NEW HAVEN, CONN.

1, Salt-marsh peat; 2 , fresh-water peat; 3 , contact.

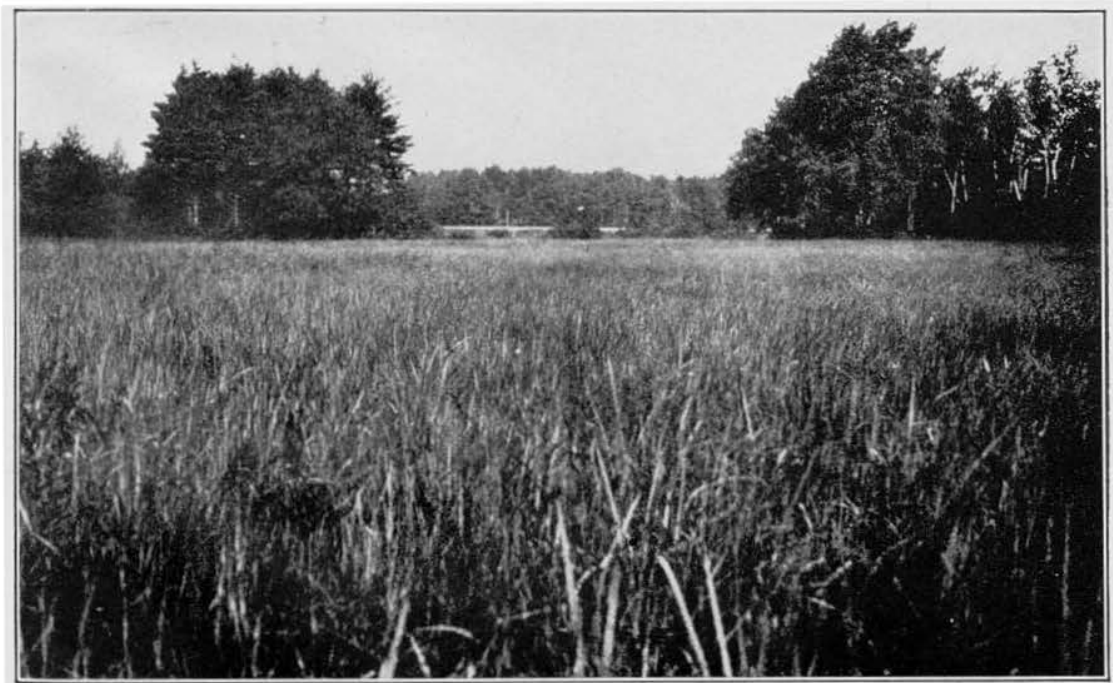

B. TYIICAL PEAT MEADOW NEAR LOWELL, MASS. 

stand in place in the excavations at the bottom of the fresh-water peat. Specimens were taken from the exposed section, which is oneeighth of a mile long, and from a hole put down a few yards from the clay pit.

Analysis 53 (p. 19) shows the composition of a combined sample of fresh-water and salt-water peat taken from the hole, and analysis 54 (p. 19) that of a sample taken from a pile of peat which had been exposed to the weather for a year. The high ash content shown in analysis 54 is ascribed to the admixture of dust from the adjoining clay pit. Analyses 53a-d (p. 19) show the composition of samples of both the salt-marsh and fresh-water peat collected by George E. Nichols.

Thickness of beds of salt-marsh and fresh-water peat in boring in marsh on Quinnipiac River, Conn., sampled for analysis.

$\begin{array}{ll}\text { Salt-marsh peat (analysis 53a) } & \text { Feet. } \\ \text { Salt-marsh peat (analysis 53b) } & 1 \frac{1}{2} \\ \text { Fresh-water peat (analysis 53c) } & 1 \\ \text { Fresh-water peat (analysis 53d) } & \end{array}$

Both types of peat contain an unusually large percentage of sulphur. The average nitrogen content of the salt-marsh peat exceeds 2 per cent.

Several years ago peat from this deposit was used for fuel, but the plant has been dismantled. Much of the peat in the vicinity of the brickyard has been stripped from the land in order to obtain the underlying clay. If an attempt is made to produce fuel from this deposit the top layer of impure, silty salt-water peat should be removed to a depth of at least 2 feet.

Locality 7.-A salt marsh about 1 mile west of Short Beach and 8 miles southeast of New Haven, which is shown on the map of the New Haven quadrangle, published by the United States Geological Survey, contains a deposit about 200 acres in area and 6 feet in average thickness, which should be capable of yielding approximately 240,000 short tons of air-dried muck. Five test holes were made along a line 100 yards east of and parallel to an electric railway track that crosses the marsh. Analysis 55 (p. 19) shows the composition of a composite sample taken from these holes and indicates that the deposit is of no value for fuel or fertilizer.

Locality 8.-A deposit near New Haven, about one-fourth of a mile east of Granniss Corner, which is shown on the map of the New Haven quadrangle, published by the United States Geological Survey, is about 75 acres in area and $6 \frac{1}{2}$ feet in average thickness. It is estimated that this deposit is capable of yielding 97,500 short tons of air-dried peat. 
The peat is of the built-up type and is dark brown, well decomposed, plastic, and free from woody components. A composite sample (see analysis 56, p. 19) was obtained by mixing peat taken at intervals of 1 foot in depth from test holes put down along a line through the center. Although physically suitable for fuel, the peat in this deposit is so high in ash that only low-grade fuel could be made from it.

Locality 9.-A salt marsh at the mouth of Stony Creek is crossed by the tracks of the New York, New Haven \& Hartford Railroad and by a suburban electric railway. The deposit is about 200 acres in area and 5 feet in average thickness and should yield approximately 200,000 short tons of air-dried peat and muck. A series of test holes gave the following results:

Logs of test borings in salt marsh at mouth of Stomy Creek, $2 \frac{1}{2}$ miles east of Branford, Conn.

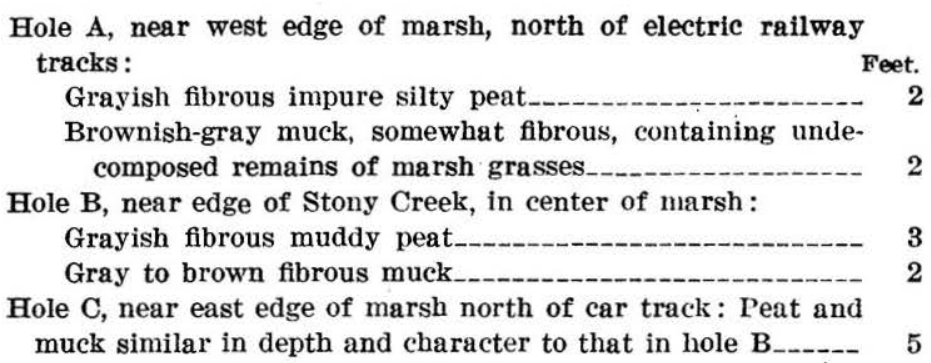

As the underlying muck is too impure to be of commercial value only the upper 2 feet of peat was sampled. A composite specimen (see analysis 61, p. 19) was taken from the sides of a small drainage ditch that crosses the marsh. The high content of ash is believed to be partly due to inorganic impurities mixed with the peat in the excavation of this ditch.

Data relating to the area, depth, and chemical composition of several other peat deposits in this county are given in the table of analyses (pp. 19-22).

RHODE ISLAND.

The most valuable peat deposits in Rhode Island occur in the fresh-water bogs and swamps of Bristol, Providence, and Washington counties. Although there are many salt marshes on the coast they contain little peat of economic value. The peat of Rhode Island is similar in origin and physical and chemical properties to that of Connecticut and other New England States. The swamps and bogs of Rhode Island are estimated to be capable of yielding $1,000,000$ short tons of air-dried peat. 
BRISTOL COUNTY.

Locality 1.-A deposit about half a mile west of Barrington, which is shown on the map of the Narragansett Bay quadrangle, published by the United States Geological Survey, was formerly about 300 acres in area, but the peat has been stripped from 200 acres in order to obtain the underlying clay. The average thickness of the peat in the remaining 100 -acre tract is only about $2 \frac{1}{2}$ feet. It is dark brown to black, rather fibrous, and earthy. The deposit was originally wooded, but it has been cleared and drained. Analysis 52 (p. 49) shows that the peat is too high in ash for use as fuel and too low in nitrogen for use as fertilizer. Other deposits in Bristol County are reported to contain high-grade peat, but they were not tested.

PROVIDENOE COUNTY.

Locality 2.-A meadow adjoining Cherry Brook about a mile south of Woonsocket, which is about 200 acres in area, contains black muck and impure peat from 1 to $3 \frac{1}{2}$ feet deep. Four test holes were put down along a line through the center of the meadow, from which a composite sample was taken. Analysis 49 (p. 49) shows the chemical composition of this sample of the deposit, which consists largely of muck.

Locality 3.-A marsh adjoining Blacksione River between Valley Falls and Lonsdale, which is about 200 acres in area, contains only sandy muck.

Locality 4.-A swamp about $1 \frac{1}{2}$ miles northwest of West Greenville, which is shown on the map of the Burrillville quadrangle, published by the United States Geological Survey, contains a deposit which is about 50 acres in area and which exceeds 25 feet in maximum thickness. If the average depth is 15 feet the deposit should yield approximately 150,000 tons of air-dried peat and muck. The living vegetation consists chiefly of maple, elm, ash, and alder trees. The peat is composite in origin and ranges in texture from fibrous to plastic. Two test holes put down in the center of the swamp adjoining a road that crosses the deposit gave the following results:

Log of test borings in swamp nearWest Greenville, R. I.

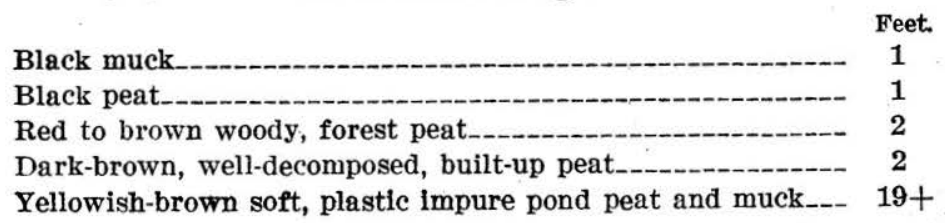

Analysis 51 (p. 49) shows the character of a composite sample obtained from these holes and indicates that the deposit is unfit for furel. This is probably the deepest peat deposit in Rhode Island, but it is not favorably situated for commercial use. 
WASEINGTON DOUNTY.

Locality 5.-A marsh adjoining Chapman Pond, about 2 miles east of Westerly, which is shown on the map of the Stonington quadrangle, published by the United States Geological Survey, is approximately 600 acres in area, but on account of high water the large tract south of the pond could not be sampled. The tract north of the pond is about 50 acres in area and contains a deposit 6 feet in average thickness, which should yield about 60,000 tons of airdried peat. If the peat in the large area south of the pond is of the same average thickness the entire deposit contains approximately 720,000 tons of peat. The peat near the pond is of the filled-basin type, but that more remote is of the built-up type. The living vegetation consists chiefly of an inner zone of cat-tails, sedges, and grasses and an outer margin of poplar, elm, and willow trees. A series of test borings gave the following results:

Logs of test borings near Chapman Pond, Westerly, R. I.

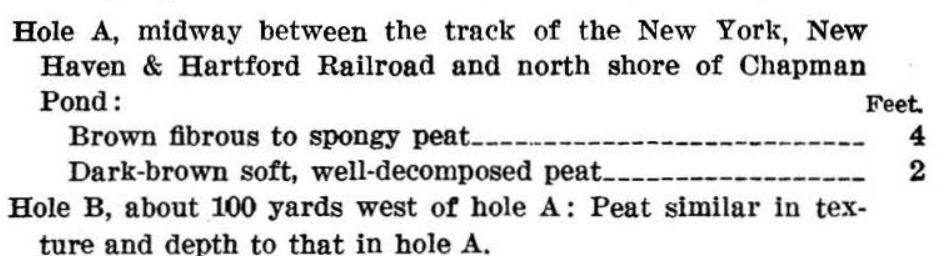

A composite sample (see analysis 50, p. 49) taken from these holes shows the quality of the peat in the northern part of the deposit.

Locality 6.-Several test holes were put down in a bog about 3 miles east of the railroad station at Westerly and 1 mile east of Chapman Pond, which forms an arm of the large bog adjoining Pawcatuck River (see locality 7). These test holes were put down at intervals of about 100 feet near the railroad track that crosses the bog but failed to show peat. The deposit appears to consist of 12 to 16 inches of black muck underlain by sand.

Locality 7.-In the part of the bog that adjoins Pawcatuck River about 4 miles west of Bradford the living vegetation consists chiefly of the cranberry, heath surubs, sphagnum, grasses, and sedgesplants that grow on typical peat bogs-but no valuable peat was found. Maple, elm, and ash trees constitute the marginal vegetation. The surface consists of a layer of muck about 2 feet deep.

Locality 8.-A swamp on Pawcatuck River about 1 mile east of locality 7, which is shown on the map of the Stonington quadrangle, published by the United States Geological Survey, is densely wooded, and in August, 1918, was covered with water from 6 to 12 inches deep. No peat of economic value was found. A deposit of undecomposed plant remains and black muck about 1 foot thick constitutes the surface layer. 
Locality 9.-Indian Cedar Swamp, which is shown on the map of the Charlestown quadrangle, published by the United States Geological Survey, lies about 1 mile south of the railroad station at Wood River Junction. This swamp is about $1 \frac{1}{2}$ miles long and 700 acres in area. Samples were taken around the margin, but on account of the surface water the interior was not tested. In the part sampled only 1 foot of muck was found. The swamp is densely forested. Peat of workable depth may occur in the interior, but such extensive clearing and road building would be necessary that the present value of the deposit is doubtful.

Locality 10.-Great Swamp, between West Kingston and Kenyon, in August, 1918, was mostly flooded. Where it was examined around the margin no peat of economic value was found. Deep peat is reported to occur in the interior of the swamp, but this report was not confirmed.

\section{OTHER STATES.}

Conditions are favorable for the formation of peat in the upper valley of Red River, in North Dakota, and in the region east of James River, in South Dakota, but these areas were not examined for this report. Small accumulations of muck and peat have been noted in Lake Marsh, Hamlin County, and Madison Lake, Lake County, S. Dak., but the material in these localities probably contains a large proportion of inorganic impurities and may not be of workable depth.

\section{ATLANTIC COASTAL REGION.}

VIRGINIA AND NORTH CAROLINA.

GENERAL FEATURES.

Although some peat is found in the tidal flats on Potomac River, the most extensive deposits in Virginia occur in the Dismal Swamp district, which extends southward from Portsmouth into the northern counties of North Carolina. Peat also occurs throughout an area about 50 miles wide along the coast of North Carolina from Albemarle Sound to the northeastern boundary of South Carolina, as well as in parts of the upper valleys of Roanoke, Neuse, and Cape Fear rivers. The peat, which is largely of the built-up type, originated in relatively flat, undrained salt marshes along the coast and in freshwater swamps farther inland. The deposits of Virginia and North Carolina are estimated to be capable of yielding 700,000,000 short tons of air-dried peat. The Dismal Swamp lies in both Virginia and North Carolina and contains most of the peat in these States, and therefore the two States are considered under one heading.

ARIINGTON AND FAIRFAX COUNTIES, VA.

There are several small undrained areas in the eastern parts of Arlington and Fairfax counties, Va., adjoining Potomac River. 
The marshes south of Alexander Island, at the mouth of Fourmile Run, and in Hunting Creek, as well as the tidal marsh about 2 miles south of Alexandria, were examined, but only shallow bluish-gray impure peat and silty muck, consisting largely of the remains of marsh grasses, were found. These areas are illustrated on the map of Washington and vicinity, published by the United States Geological Survey. The tidal marsh south of Alexandria is nearly 2 miles in length and ranges from about one-eighth to one-half mile in width. Its surface lies below the water level of Potomac River at high tide and consequently it is usually covered with water from a few inches to about 2 feet in depth. The living vegetation consists largely of wild rice, flags, and marsh grasses. If this marsh were drained the soil would probably yield good truck crops, but it seems doubtful whether drainage would be profitable. Similar marshes occur at the mouths of numerous tributaries to Potomac River in other counties.

\section{DISMAL SWAMP DISTRICT.}

GEOGRAPHY AND GEOLOGY.

The Dismal Swamp district is in the Coastal Plain of southeastern Virginia and northeastern North Carolina. (See Pl. XVI.) It lies roughly between parallels $36^{\circ} 15^{\prime}$ and $36^{\circ} 45^{\prime} \mathrm{N}$. and meridians $76^{\circ} 5^{\prime}$ and $76^{\circ} 35^{\prime}$ W., and approximately includes Norfolk County and the eastern part of Nansemond County, Va., and Perquimans, Pasquotank, Camden, and Currituck counties, N. C. As the limits of the swamp depend largely upon rainfall and vegetation, as well as topography, they are rather irregular and are not sharply defined.

The swamp is traversed from Deep Creek, Va., to South Mills, N. C., by the Dismal Swamp Canal and is cut by numerous smaller canals and ditches radiating from Lake Drummond. The Norfolk Southern Railroad skirts the eastern, southern, and western parts of the swamp, and its north end is crossed by the Virginian, Seaboard Air Line, and Norfolk \& Western railroads.

The total area of the Dismal Swamp is about 2,200 square miles, of which a little more than 700 square miles has been drained by the Dismal Swamp Canal and other ditches. A large part of the swamp is owned by the Roper Lumber Co., Norfolk, Va., and by the Richmond Cedar Works, Richmond, Va.

The region as a whole is but sparsely populated, and the chief industries in the reclaimed areas are lumbering and agriculture.

The peat deposits of the Dismal Swamp lie in shallow basins that originated in an extensive depression of the Columbia group of formations. During the deposition of these formations the mouth of James River was some distance southwest of its present mouth, ${ }^{54}$

${ }^{54}$ Darton, N. H., U. S. Geol. Survey Geol. Atlas, Norfolk folio (No. 80), pl. 1, 1902. 


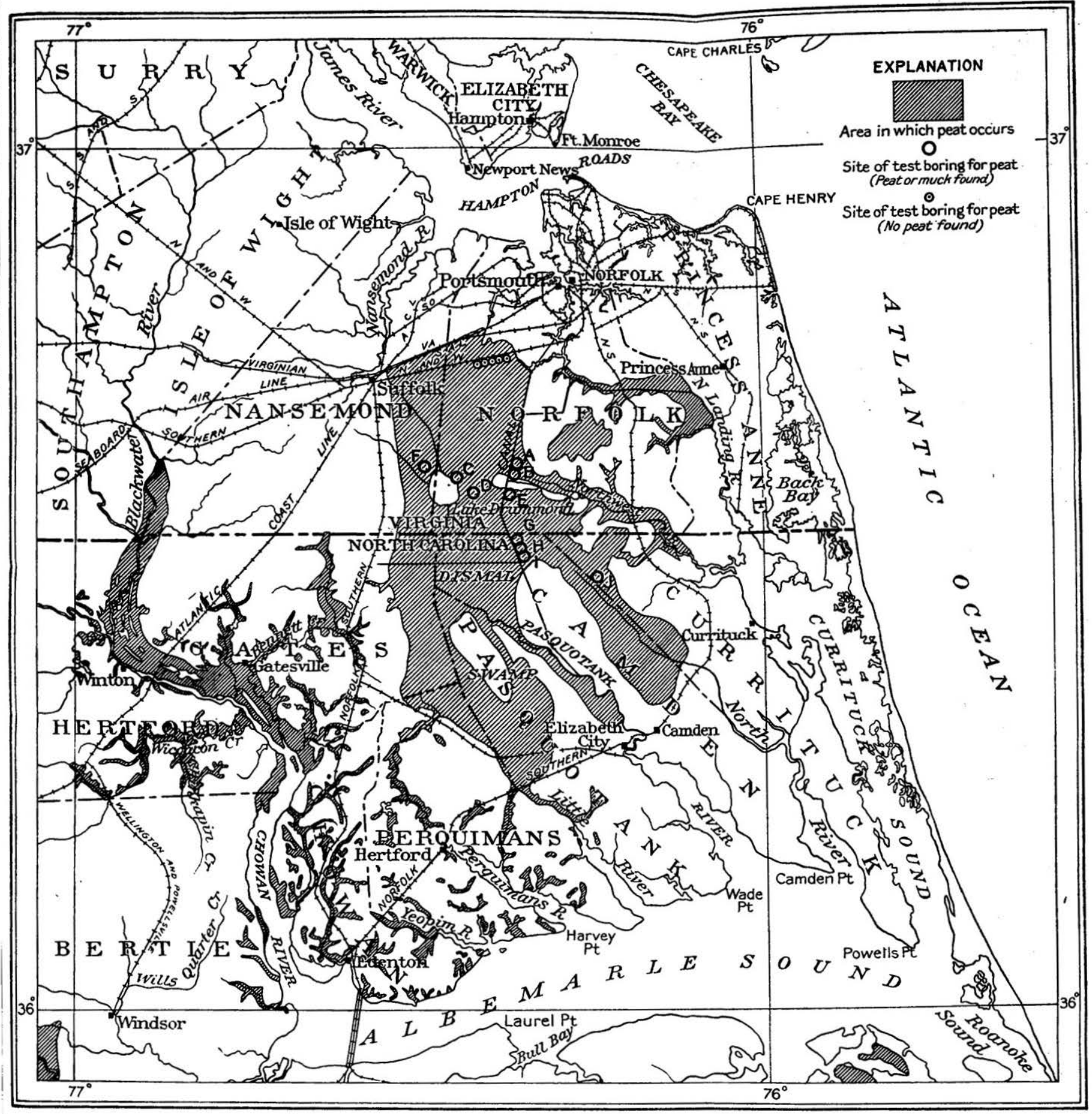

$10 \quad 0$

10

20

30 Miles

MAP OF SOUTHEASTERN VIRGINIA AND NORTHEASTERN NORTH CAROLINA SHOWING DISTRIBUTION OF PEAT AND LOCATION OF TEST BORINGS IN THE DISMAL SWAMP 

and the sediment it laid down probably formed a bar or delta east of the swamp. When the land was subsequently uplifted, the mouth of the James was diverted to its present position, and between the terrace formed by its delta and the Nansemond escarpment there remained a large, poorly drained depression in the surface. The normal precipitation of the region is about 52.08 inches and the average humidity is 73 per cent. Thus favored by topographic and climatic conditions, the surface was soon saturated or covered with water and luxuriant vegetation, and it has remained in substantially the same state since that time.

While the swamp was young most of the peat was formed below water level and the deposits were largely of the filled-basin kind. Later many basins in the region were filled to the general level of the surrounding country by vegetal accumulations, and much of the surface water was drained off through the Dismal Swamp Canal and subsidiary ditches. Thus the Green Sea, which was originally connected with the main morass, has been detached by the draining of the intervening area. Many marginal sections have also been reclaimed for agricultural use by small ditches and are no longer swampy, except in very wet weather. However, the greater part of the swamp is still so poorly drained and so choked with plant growth that it is continually saturated or covered with water. The average depth of the water is only a few inches. In the western part the water is in places 2 feet deep, and peat is still forming there under water. In the eastern part the water seldom stands above ground, although in many places it keeps the surface so highly saturated that peat is still accumulating. In the thoroughly drained sections the formation of peat has been superseded by that of leaf mold.

FLORA.

The Dismal Swamp lies at the junction of the coniferous and deciduous forest regions of the eastern coast of the United States. Its flora comprises plants of a great number of species and is interesting because it shows a mingling of the northern and southern land floras. In the earlier stages of peat formation algae and mosses probably grew profusely in its shallow waters, building up deposits of fine-grained peat, which are now found in the bottoms of the basins. As the remains deposited by these plants accumulated the basins became shallower, enabling the pondweeds (Potamogeton), the water lilies (Castalia and Nymphaea), and the lake bulrush (Scirpus) to establish themselves temporarily. ${ }^{55}$ In some of the higher parts of the morass the bog-meadow and bog-heath stages, in which the Carex and Andromeda-Ledum associations predomi-

ss Davis, C. A., U. S. Geol. Survey Bull. 376, p. 13, 1909. 
nated, may have followed in small areas. As the surficial depressions were relatively shallow, and as logs are found in nonfibrous peat at depths of 5 to 10 feet in many sections of the swamp, it is believed that deciduous and coniferous trees more or less fully superseded the other flora at an early date and contributed the greater part of the dead vegetation from which the peat deposits of the region were formed.

The present flora of the Dismal Swamp includes aquatic plants, the fern and peat-moss association, deciduous and coniferous trees, and associated undergrowth. Nearly the whole region is forested, and the plant associations are so intimate that it is difficult to delimit the distinct formations. However, the following ecologic classification, which follows roughly the classification used by Schenck in "Biologie der Wassergewächse," shows the most abundant plants that are now contributing to the formation of peat:

Submersed:

Utricularia spp.

Riccia fluitans.

Philotria canadensis.

Sphagnum kearneyi.

Callitriche heterophylla.

Juncus repens.

Isnardia palustris.

Floating on the surface:

Spirodela polyrhiza.

Castalia odorata.

Nymphaea advena.

Aquatic plants.

Floating on the surface:

Nelumbo lutea.

Potamogeton lonchites.

Callitriche heterophylla.

Rising above the surface:

Sparganium androcladum.

Myriophyllum heterophyllum.

Woodwardia virginica.

Eriophorum virginicum.

Decodon verticillatus.

Limodorum tuberosum.

Sphagnum cymbifollum.

Black gum association.

Water ash (Fraxinus caroliniana).

Rattan (Berchemia scandens).

Yellow jessamine (Gelsemium semper-

virens).

Cross vine (Bignonia capreolata).

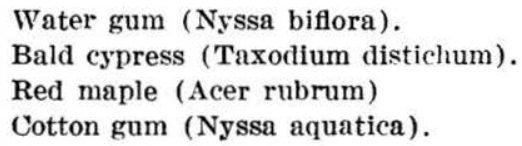

White cedar association.

Loblolly pine (Pinus taeda).

Sweet bay (Magnolia virginiana).

White cedar (Chamaecyparis thyoides).

Shrubs (Ericaceae association).

Cane (Arundinaria macrosperma).

Aquatic plants are found in nearly all the wetter parts of the swamp, notably in some of the abandoned ditches, which have been completely filled with them. However, in areas of dense shade they make little headway and are of minor importance in the formation of peat. 


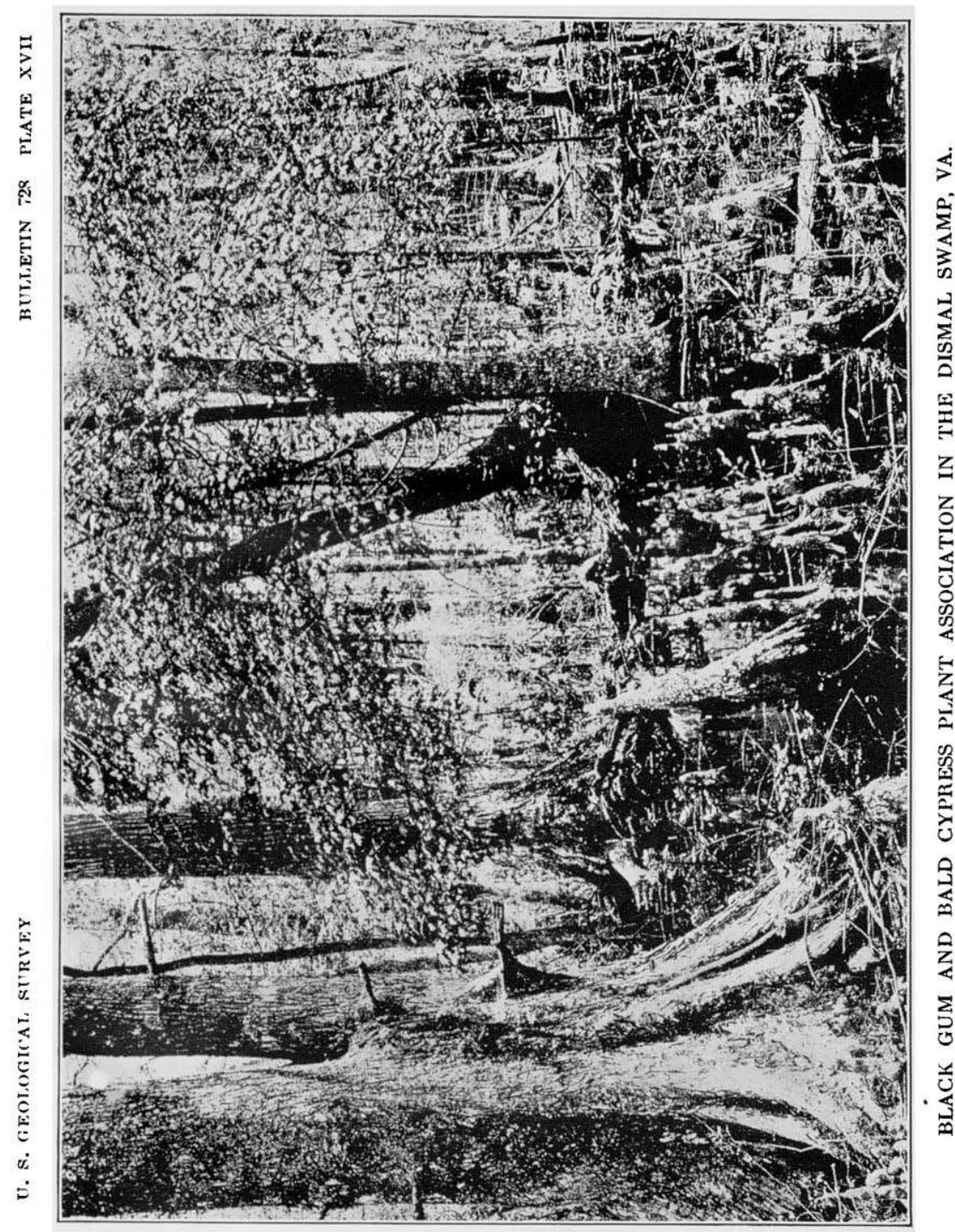



Ferns and peat mosses are found in the open parts of the region. Sphagnum cymbifolium grows in the shallow water and among the stipes of the Woodwardia in the higher parts of the peat area. The roots are usually submerged, and the stems, which range in length from 6 to 18 inches, rise above the surface.

The water gum, locally known as black'gum, and white cedar or juniper forest associations predominate throughout the region and contribute the greater part of the dead vegetation that is now accumulating. The densely forested wetter part of the morass, especially the area surrounding Lake Drummond, is known as the blackgum swamp. (See Pl. XVII.) It is characterized by the profuse growth of black gum and its associated undergrowth, although red maple is also abundant. Here immense quantities of peat are now accumulating. The bald cypress is also still found in some parts of the black-gum swamp but probably was more abundant in earlier years. In fact, the most striking feature of the region is the weird aspect presented by the cypress knees in the interior and the belt of old weathered cypress stumps standing on the margin of Lake Drummond and hung with Spanish moss. These stumps, which have survived the attacks of the weather for many years, and the numerous well-preserved cypress logs that are encountered several feet beneath the surface indicate that cypress formerly contributed much more dead regetation to the peat deposits than now.

The open, light parts of the morass, known locally as juniper swamp, are largely occupied by the white-cedar association. In earlier years these areas were completely forested by white cedar, but now, owing in part to the work of man, many of them bear a growth of shrubs and cane.

PROPERTIES.

The two leading kinds of peat in the Dismal Swamp are known locally as "black-gum peat" and "juniper peat." The "black-gum peat," which is dark brown or black, thoroughly decomposed, and relatively homogeneous in structure, is found in what were formerly the wetter parts of the region, especially near Lake Drummond, and wears a growth of black gum, red maple, and bald cypress. It is well humified and almost destitute of fibrous structure. When dry it breaks easily, leaving lusterless fracture surfaces. "Juniper peat," which ranges from dark to light brown in color and is rather fibrous, is found in the light or open swamp and bears a growth of white cedar, pine, sweet bay, shrubs, and cane. Decomposition is not far advanced and the peat contains many stems, roots, and logs. On the eastern margin of the swamp near the source of Northwest River there is a typical area of this material. When dry it hardens in lump form and breaks with difficulty. 
The chemical composition of peat in the Dismal Swamp is shown by the analyses given in the table (p. 50). "Black-gum peat" (samples 1,2, and 5), because of its thorough decomposition, contains more nitrogen and fixed carbon than "juniper peat" (sample 4) and therefore is less acidic. It also contains less ash and is of greater commercial value. Where the ash content exceeds 8 per cent it consists chiefly of alumina and silica in the form of clay and sand.

ASSOCIATED MARL.

Shell beds or so-called marls underlie the peat deposits at many places in nearly all the counties of the Dismal Swamp region. Although no outcropping beds were observed in the areas tested for peat, it is said that many of these strata have been penetrated by wells and extensively exposed by drainage excavations. Shells thrown upon the bank of Dismal Swamp Canal by dredges were seen near Deep Creek and Wallaceton, Va., and Lilly and Moyock, N. C. Several years ago a dredge of the Lake Drummond Canal \& Water Co. in deepening the canal feeder penetrated a shell bed about midway between the source and mouth of the feeder. A large quantity of shell "marl" was thrown upon the bank at this point, but on account of the action of the dredge and of the weather since that time it is now disintegrated and mixed with sand and clay. W. C. Mansfield, who identified the following fossils collected at this locality, believes that they are of Pleistocene origin:

Venus mercenaria Linné.

Ostrea virginica Gmelin.

Arca transversa Say.

It has been said ${ }^{58}$ that the age represented by these fossils is Pliocene, but as they are also found in the Pleistocene and underlie peat of late Pleistocene origin, it is probable that they belong to the Pleistocene series.

An estimate of the quality of shell "marl" in the Dismal Swamp region is not available, but if the material occurs in workable quantities and can be cheaply excavated, many peat areas in this region that on account of the acidity of the soil are now valueless for general farming could perhaps be economically treated with lime from these shell beds and made to yield large alkaline-soil crops.

DISTRIBUTION AND QUANTITY.

The Dismal Swamp covers approximately 2,200 square miles, of which a little more than 700 square miles has been permanently drained to a depth of 3 feet or more by Dismal Swamp Canal and smaller ditches. (See Pl. XVI.) Much of the drained land is

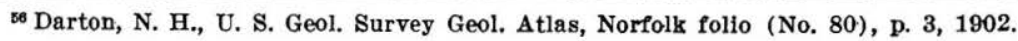


farmed. In the remaining 1,500 square miles peat deposits ranging in depth from 1 foot to 20 feet are found. The thickest beds lie in the region east and northeast of Lake Drummond, where peat 18 feet deep was exposed by comparatively recent excavations. The peat in this area is black and low in inorganic impurities and is probably the best in the swamp. In general, the depth of the peat gradually decreases toward the edge of the swamp, where the peat finally merges into the sands of the adjoining areas. The eastern border is deeply indented by large tracts that have been drained, cleared, and cultivated. Some valuable peat is found in the southern and southeastern parts of the morass, but the northern and western parts contain few deposits large enough to be of commercial importance.

The Dismal Swamp is not entirely covered with peat-in fact, not more than half of it contains peat of commercial value. In some parts of the swamp the peat is too shallow to be worked profitably, and in others it contains so many roots, stumps, and logs that excavation by present methods would be impracticable. In many areas the peat has been destroyed by forest fires. In some of the heavily forested areas peat has never formed to great depths, perhaps because of the presence of excessive surface water and dense shade, which prevented the growth of shrubs, mosses, grasses, reeds, ferns, and other prolific peat-forming plants. From numerous test borings, observations made along the banks of drainage canals, and information furnished by drainage engineers who are familiar with the swamps, it is estimated that the average thickness of the peat is 7 feet. On the assumption that the uncultivated area of the Dismal Swamp is 1,500 square miles, that about one-half of this area is covered with peat averaging 7 feet in depth, and that the bog will yield 200 tons of dry peat per acre-foot, then the total available peat in the Dismal Swamp is $672,000,000$ tons.

AREAS TESTED.

Norfolk County, Va.-The best peat in the Dismal Swamp is found in Norfolk County, Va., in the territory northeast of Lake Drummond. This area is close to the truck-farming section of Virginia, to which the peat could be cheaply transported by means of the Dismal Swamp Canal. As shown by the analyses (p. 50) much of this peat is high in thermal value and contains relatively little ash and a fair percentage of nitrogen. It could therefore be used for fuel or as a nitrogenous ingredient of commercial fertilizer.

It is reported that in 1860 a peat-fuel plant was erected near the site of hole A, described below, but that on account of economic conditions which arose with the outbreak of the Civil War it was unsuccessful, and the machinery was dismantled. So far as known this 
is the only attempt that has ever been made to market peat from the Dismal Swamp.

As shown on Plate XVI, test borings for peat were made along the Norfolk \& Western Railway, the Dismal Swamp Canal, the canal feeder, and the eastern shore of Lake Drummond. No peat of consequence was found in the region traversed by the railway, but in the other areas the test borings gave the following results:

Hole A was drilled at a point 1 mile north of Wallaceton and 300 feet east of Dismal Swamp Canal. Nonfibrous peat, ranging in color from dark brown to black, was found to a depth of 10 feet. Sam. ple 1 was taken at a point 4 feet from the surface, and sample 2 at 6 feet. (See analyses, p. 50.)

Hole B was made on the north bank of Northwest River, 300 feet east of Dismal Swamp Canal. Here 9 feet of peat similar in physical characteristics to the specimens taken from hole A was found. Samples 3 and 4 were taken at depths of 4 and 6 feet, respectively.

Hole $\mathrm{C}$ was sunk to a depth of 9 feet at a point midway between Jericho Canal and the source of the feeder ditch by which water is supplied to Dismal Swamp Canal. About 8 feet of peat, underlain by white sand, was found here, but as it seemed to be similar to the material from holes A and B no sample was taken for analysis.

Hole D, in which 10 feet of peat underlain by alternate layers of white sand and blue clay was found, was made 200 feet north of the water gate in the Dismal Swamp Canal feeder. The surface layer of this peat is black and well decomposed, but the subsurface layers range in color from dark to light brown. Sample 5 was taken 4 feet below the surface.

Hole $\mathrm{E}$ was drilled on the bank of Dismal Swamp Canal opposite the mouth of the feeder. As the peat was rather fibrous and only 3 feet deep at this point it was not sampled for analysis.

Nansemond County, Va.-Hole F was sunk on the south side of Washington Ditch about a mile northwest of Lake Drummond, in Nansemond County. This boring, as well as four others at intervals of three-quarters of a mile northwestward along this ditch, failed to show peat in commercial quantities. From 1 to 3 feet of muck was found, and sample 6, consisting of a composite mixture of material from the surface to a depth of 3 feet, was taken in order to show its character. The absence of peat in this area may be due to the dense stand of mature timber and the excessive quantity of water that cover it, preventing the growth of shrubs, mosses, and other prolific peat-forming plants.

Currituck County, N. C.-Hole J, in Currituck County, N. C., was put down on the north side of Old Swampy Road about 800 . yards northeast of the line between Currituck and Camden counties. It penetrated 7 feet of black, thoroughly decomposed peat. The 
area of this deposit is approximately 100 square miles, and for the most part the material is of the built-up type, though the deposit contains some filled-basin peat. As shown by the analyses (p. 50), the peat is unusually high in thermal value, contains little ash and a relatively high percentage of nitrogen, and seems to possess much value as a source of fuel and fertilizer.

Camden County, N. C.-Holes G, H, and I, which were sunk half a mile apart along Dismal Swamp Canal, and half a mile to $1 \frac{1}{2}$ miles northwest of Lilly, Camden County, N. C., failed to show peat of commercial value. Muck averaging 3 feet in depth was found, but it was not sampled. These tests, however, do not prove that there is no peat in workable quantities in the county. The vegetation in the region consists chiefly of maples, sedges, grasses, canes, ferns, and mosses. Sphagnum moss suitable for surgical dressings is abundant, and it is reported that pure sphagnum covers mant square miles in the territory west of Dismal Swamp Canal.

Other counties.-Large quantities of peat are said to occur in Perquimans and Pasquotank counties, N. C., but they were not examined for this report.

\section{SOUTH CAROLINA.}

Although peat occurs in marshes and swamps adjoining Peedee, Santee, and Combahee rivers and in salt marshes along the coast, notably in Georgetown and Horry counties, many of the deposits are small and shallow and contain a large proportion of inorganic impurities. According to Sloan, ${ }^{57}$ the ash content of the peat where tested in the counties mentioned is about 29 per cent, which shows that it is of no value for fuel.

GEORGIA.

Peat is found near Pineora, Effingham County, in the Okefenokee Swamp, and at other places in the southeastern part of Georgia. The deposit near Pineora is reported to be about 600 acres in area and 5 feet deep and to be suitable for fertilizer.

\section{FLORIDA.}

General features.-Peat deposits are distributed over almost the entire State of Florida, and that State probably contains more peat than any other, except Minnesota and Wisconsin. The deposits that have been examined appear to contain 1,000,000,000 tons, and the Everglades, which have not been extensively tested, probably

8n Sloan, Earle, Catalogue of the mineral localitles of South Carolina: South Carolina Jeol. Survey Bull. 2, ser. 4, p. 362, 1908.

$91065^{\circ}-22-14$ 
contain an equal quantity. The State is therefore believed to be capable of yielding $2,000,000,000$ short tons of air-dried peat.

For the sake of convenience the deposits are roughly assigned to the following 14 regions, illustrated by Plate XVIII, and though these regions are largely geographic, the peat shows some differences in origin and quality. The data here given were taken in substance from a report by the Florida State Geological Survey. ${ }^{58}$ For analyses of peats of Florida see pages 23-26.

West coast region.-The west coast region includes a belt of land a few miles wide along the western coast, which contains small quantities of good peat in depressions among sand dunes. Some peat is also found in the estuaries of Blackwater, Apalachicola, and other rivers, but these deposits contain so much inorganic material that they are of little economic value.

West, pine-hill region.-The climatic and floral conditions in the west pine-hill region are favorable for the formation of peat, but the topography is generally unfavorable. However, some peat of fair quality is reported to occur in some of the Tyty swamps.

West limestone region.-The quantity of peat in the west limestone region is small.

Middle hammock region.-Because of the few streams and the thorough drainage of the basins in the middle hammock region it contains relatively little peat.

Lime-sink region.-The lime-sink region does not contain much peat.

Middle flatwood region.-Numerous deposits of shallow peat occur in the ponds throughout the middle flatwood region.

Gulf hammock region.-Large peat deposits occur in lakes, rivers, and estuaries in the Gulf hammock region. The best deposit thus far tested is that at Lake Panasoffkee, Sumter County, which contains peat 20 feet deep of good quality for fuel or fertilizer.

Lake region.-Thousands of small lakes containing extensive peat deposits occur throughout the lake region. In fact, peat of excellent quality is said to be found in nearly every square mile. The swamps and bogs are of different types, and consequently the peat varies in composition.

East flatwood region.-The east flatwood region contains extensive peat deposits in the estuary of St. Johns River. As the river is navigable, the peat could readily be transported to market. The predominant vegetation in this region consists of long-leaf and slash pine, saw palmetto, pond cypress, and sweet and black gum trees.

East coast region.-In a narrow region along the eastern coast of Florida from the northern boundary to Cape Florida, a distance of

s $\mathrm{Harper,} \mathrm{R.} \mathrm{M.,} \mathrm{Prellminary} \mathrm{report} \mathrm{on} \mathrm{the} \mathrm{peat} \mathrm{deposits} \mathrm{of} \mathrm{Florida:} \mathrm{Florida} \mathrm{Geol.}$ Survey Third Ann. Rept., pp. 197-375, 1910 

more than 350 miles, barrier beaches, sand dunes, and lagoons containing fresh and salt water are common. Many of the fresh-water lagoons contain good peat.

South flatwood region.-The south flatwood region embraces the northern part of the Everglades and contains one of the most extensive peat deposits in the United States.

Miami limestone region.-The Miami limestone region, which includes part of the Everglades, contains many peat deposits, most of which are of swamp and estuarine origin.

Coast prairie region.-The peat resources of the coast prairie region are reported to be relatively scanty.

Key region.-The key region, though it is not a commercial source of peat fuel, is interesting because most of its peat was formed by the decomposition of débris from mangrove trees.

\section{OTHER STATES.}

As shown on Plate I, peat occurs in areas in southern Delaware and southeastern Maryland, but they were not examined for this report.

\section{OTHER REGIONS.}

GULF COAST.

Peat occurs in a narrow belt of land along the coast of Alabama, Mississippi, Louisiana, and Texas, but so far as the writers are aware, the deposits have not been examined.

\section{CALTFORNIA.}

Although California contains several large deposits of impure peat and muck, areas of undrained land in that State are relatively small compared with those of the States in the glacial and Atlantic coastal regions that contain the most notable deposits of peat. The principal deposits occur in Lower Klamath Lake, Siskiyou County, in the lower valleys of Sacramento and San Joaquin rivers, and in Los Angeles and Orange counties. Some peat is also found in San Bernardino County and in the marshes adjoining San Francisco Bay and Salinas River. Many of the valley deposits, especially those near Sacramento and Stockton, contain so much sand and silt that has been deposited by the rivers and are so deeply buried by hydraulic mining débris that they are of doubtful value for fuel, but much of this peat contains a high percentage of nitrogen and could be advantageously used as a direct fertilizer or as an ingredient of commercial fertilizers. The peat lands in the lower valleys of Sacramento and San Joaquin rivers are very fertile and bring high prices when sold for agricultural use. 
The following excerpt ${ }^{59}$ contains the best data available relating to the distribution and quaritity of peat in California:

The large undrained river valleys of California are overgrown by tule, duck weeds, cat-tails, and other plants, and should therefore contain peat deposits. The deepest deposits are believed to occur in the area extending from Carquinez Straits to Stockton and Riovista, embracing 12 townships, 432 square miles. or 276,480 acres. The peat probably ranges from 6 to 80 feet in depth, or an average of 43 feet. The eastern part has been covered by debris from hydaulic mines, and in other portions the peat is somewhat sandy. Probably about one-half of the total area, or 138,240 acres contains peat. Of this area 60,000 acres are known to contain good peat to a depth of at least 6 feet. This tract is therefore estimated to be capable of ylelding $72,000,000$ short tons of air-dried peat. If the entire 138,240 -acre tract contains peat 43 feet in average depth it should yield $1,188,864,000$ tons, but it is probable that most of the material outside of the 60,000 -acre tract is muck. It is unfortunate for the peat industry that this land is among the most valuable in California for agricultural purposes, as it is therefore questionable whether the peat would prove more valuable for fuel or fertilizer than for farming.

OREGON AND WASHINGTON.

The topography of Oregon and Washington is unfavorable for the accumulation of large deposits' of peat. However, many small undrained areas adjoining lakes and rivers in the central and eastern parts of these States contain small peat beds, but it is doubtful whether they are of commercial extent.

Peat has been reported to occur in some of the lakes and river valleys of Cowlitz, King, Pierce, and Snohomish counties, Wash., but most of it is believed to be high in ash and unfit for fuel.

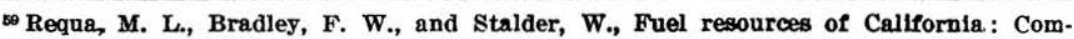
monwealth Club of San Francisco Trans., 1912. 


\section{INDEX.}

A.

Acknowledgments for ald.

Page.

Addison County, vt., deposits in. 156-15y

Alabama, limestone in

peat in

Alburg, Vt., deposits near._._._. 168-164

Alden, W. C., acknowledgment to

Allen County, Ind., deposit in_-.- 112, 113

Analyses of peat.

19-57

method followed in......... 18-19

Antiseptic dressing, use of peat as_ 4,73

Arlington County, Va., deposits in- 191-192

Ash in peat, amount and analyses

See also Analyses of peat.

B.

Bald cypress association in the Dismal Swamp, plate showing

Bandages, use of peat in

Bastin, E. S., and Davis, C. A., cited--_-_-_-_--12

Beals, E. A., acknowledgment to-_- 2 analyses supplied by

Berkshire County, Mass., deposit in - 165-166

Beyer, S. W., cited._._. 29

Big Island, N. Y., reclaimed peat s w a m p near, plate showing

Black-gum association in the Dismal Swamp, plate showIng - 194

“ Black-gum peat," nature of -- 195, 196 Bloomingdale, N. Y., deposit near_ 129-131 Bog, definition of

Botsford, Conn., deposit south of -- 184-185

Bramford, Conn., deposit near.-.- 188

Briquets, peat, process of making-- 71-72

Bristol County, R. I., deposit in

Bristol Pond, Vt., plate showing--- 156 spruce-sphagnum bog on shore of, plate showing _.. 156

Brookfleld, Mass., deposits near-- 181-182

Built-up deposit, definition of.--_-- 8 examples of formation of plant constituents of -........ 10 plate showing -

Burchard, E. F., and Elmley, W. E., cited_... 82-91

Burlington, Vt., deposits north of- 159-160 Burning of peat deposits_..... 95 Butler, R. G., acknowledgment to-_ 2 By-products, recovery of, from peat_ 72,78 c.

Prge. 201-202

California, deposits in Camden County, N. C., test borings in - 198 Canada, preparation of peat fuel in - 70-71 Carbon, content of, in peat_...-- 16 See also Analyses of peat.

Carbonization, process of Carlisle, Mass., deposits in Cat Tail Valley, Ily., deposits in -- 110-111 Cayuga County, N. Y., deposits in- 126 Celery, raising of, on peat soil_._. 63 , $126,137,146$

Cellulose, formation and decomposition of $6-7$

Charcoal, peat, former use of 72 Charles River, Mass., deposits on_ 178-179 Chemical changes in the formation of peat _- 6-7

Chemical composition of peat_....- 16-57 Cheshire County, N. H., deposits

in

Chittenden County, vt., deposits in

Cicero Swamp, Onondaga County, N. Y., de s c r ip t 1 on of

native section of, plate showing - 136

Climate, influence of, on the formation of peat.

Climbing-bog deposits, description of, by N. S. Shaler.-- 147-148

occurence of 8

Clinton County, N. Y., deposits in $126-127$

Cloth, use of peat in._. 74

Coal, formation of 7

Coke, preparation of, from peat.-- 72

Colors of peat... 15

Columbla County, Pa., deposits in- 123-124

Composite peat bed, cross section of, plate showing-_-_ 186

features of

formation of

Concord River, Mass., deposits on - 173-174

Connecticut, analyses of peat and muck from _._._. 19-22

deposits in, nature and extent of -

Consumption of peat__._._._. 75

Contoocook River, N. H., deposits on

Cooper, H. M., analyses by- $19,27,34-35$, $43,44,45,49$

Corn raised on peat land, plate showing 
Coville, Frederick V., acknowledg ment. to

Crawford County, Pa., deposit in-- 124 Cromwell, Conn., deposit north of 185 Crops suited to peat soll_____.__- 8, 68, 64 Currituck County, N. C., peat in. 198-199 Cut peat, preparation of..._. 69

\section{D.}

Dachnowski, Alfred, acknowledg. cited._- ment to

Davis, Charles A., acknowledgment to cited_- 15, 30-33, 46-47, 54-57, 97-102

Definitions of peat and muck._._. 4 Dekalb County, Ind., deposits in 112 , 113-114

Delaware, limestone in peat in - 201

Deposits, distribution of, by States 91-202 largest__.________________ 91 selection of, for working_____ 77-79 types of, plates showing _.____ 8 Dickinson County, Mich., deposit in_- 97 Disinfectant, use of peat for....- 66 Dismal Swamp, N. C.-Va., areas tested in areas tested in, map showingblack gum and bald cypress association in, plate showing - 194

flora of _geography and geology of -.- 192-193 “ marl" in _._._._._. 196 peat in, formation of _._-_ 192-193 map showing distribution of

192

nature and extent of_-_-_ 196-199

Dutchess County, N. Y., deposits in $127-128$

E.

Earthy peat, definition of East Monkton, vt., deposits near_ 157-159 Eaton County, Mich., deposit in Elkhart County, Ind., deposits in 112, 114-115

Emmons, W. H., acknowledgment to Essex County, Mass., deposits in - 166-168 Essex County, N. Y., deposits in -- 128-133 Evolution of a peat bog, plates showing

F.

Fairfield County, Conn., deposit in - - 184-185

Feed for stock, use of peat in ..._. 4,66 Bertilizer, preparation of peat for - - 61-62, selection of a deposit for $76-77,79-80$ value of peat as_..._-_ $2-3,17,59-61$ Fibrous peat, definition of.
Filled-basin deposit, definition of.-. Past 8 examples of ............... 125 formation of plant constituents of plate showing - 8

Fishkill, N. Y., deposit near-_._._ 127-128 Floras, common types of peat deposits based on...-.- , 8-9

Florida, analyses of peat and muck from

limestone in

peat in map showing locations of $\quad 200$

Franklin County, N. Y., deposits in -

Franklin County, Vt., deposits in $160-161$ Fremont, N. H., deposit near..._. 155-156 Fresh-water deposits, formation of $\quad 11$ Fuel, dry hand-cut, plate showing - 62 preparation of peat for $67,68,69-73$, $76-77,79-80$ value of peat as _._._- $3-4,18,66-69$ Fulton County, Ind., deposits in - 115-116

\section{G.}

Gas, production of, from peat

Genesee County, N.Y., deposits in - 138-135 Geologic conditions aiding the formation of peat._._. 5-6

Georgia, limestone in 84 peat in

Glaciation, influence of, on the formation of peat._...-.

Grand Isle County, vt., deposits in

H.

Haanel, B. F., cited______- 70-71

Halifax, Mass., deposit near._._._ 180

Hartford County, Conn., deposit in _- 185

Harvard, Mass., deposit near.-..-_ 183

Herkimer County, N. Y., deposits in

Hice, R. R., acknowledgment to _-_ 2

Hills, J. L., and Hollister, F. M., cited-- 161

Hillsborough County, N. H., deposits in - - 152-154

Houghton County, Mich., deposits in -

Huels, F. W., cited.

\section{I.}

IIInois, analyses of peat and muck from._. $27,58-59$

deposits in, nature and extent of -

limestone in

Indiana, analyses of peat from _..- 27-28 deposits in, nature and extent of -

limestone in Industry, peat, conditions governing - $76-80$ 
Pare.

Iowa, analyses of peat from__-_ 29,58 deposits. In - 102-104

Himestone in

Iron Cótinty, Mich.; deposits in

Isle Lá 'Motte, Vt., deposit near-- 162-163

J.

Jasper County, Ind., deposit in -.- 116 Jennings, O. E., acknowledgment to

Johnson, s. W., cited--

2

"Juniper peat," nature of

14,17 195-196

\section{K.}

Kalamazoo County, Mich., deposits in - 99-100

Kane County, Ill., deposit in_-..- 105

Kankakee County, Ill., deposit in_- 105

Kentucky, limestone in_.._...... 86, 88

Knox, G. D., cited.

Kosclusko County, Ind., deposit in- 116

\section{I.}

Lake Clear, N. Y., deposft sonth of 131

Lake County, Ill., deposits in deposits in, map showing locabution of ........- 104

Land, undrained, types of -.... 7-8

Lane, Franklin K., cited.....- 62-63

Lapeer County, Mich., deposit in.- 100

Lee County, Ill., deposits in

Lexington, Mass., deposit in.--- 171-172

Limestone, distribution of, by

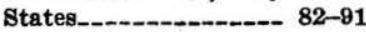

Litter, stable, use of peat for.-. 66

Littleton, Mass., deposits in _..._- 168-169

Louisiana, limestone in

peat in

Lowell, Mass., deposit near._.-169-170

peat meadow near, plate showing

186

Luce County, Mich., deposits in.-.- 100

Lyons, R. E., analyses by

\section{M.}

Maçhine peat, preparation of--.-- 69-71 Maine, analyses of peat and muck from

deposits in, map showing location of - 146

nature and extent of

Marl, deposition of

value of, on peat solls_._._._._ 82

Marquette County, Mich., deposit in - 100

Marsh, definition of - 7-8

Marshall County, Ind., deposits in.- 117

Maryland, limestone in

peat in

Mason County, Ill., deposit in

Massachusetts, analyses of peat and muck from

deposits in, nature and extent of -

Mecosta County, Mich., deposit in

Methuen, Mass., deposit near--_- 167-168
Michigan, analyses of peat and muck

Paga. from.-..--

deposits in, nature and extent of -

limestone in

northern peninsula of, map showing distribution of peat in - 96

Middleboro, Mass., deposit near-- 180-181

Middlesex County, Conn., depostts

in - $185-186$

Middlesex County, Mass., deposits in -

Minnesota, analyses of peat and muck from _._- $37-42,58$

cultivation of peat soll in.-- 64

deposits in, nature and extent of

93-94

limestone in

northern, map showing distribution of peat in 92

plates showing types of peat deposits in

Mississippi, limestone in

peat in

Mississippi Valley, lower, scarcity of peat in

Montezuma Marsh, N. Y., description of -

Moss, sphagnum, distribution and quality of

sphagnum, imports of

Mountain regions, scarcity of peat in -...-- 6 Muck, analyses of $35-43,45-48,49,50,51-57$

definition of

Mud baths, use of peat for

"Muskeg" swamps, location and extent of _._.

Muskegon County, Mich., deposit in_ 101

N.

Nansemond County, Va., test borings in

198

Neponset River, Mass., deposit on New England, distribution of limestone in

New Hampshire, analyses of peat and muck from

deposits in, nature and extent of _.

New Haven, Conn., cross section of peat bed near, plate showing -..--..--..-

New Haven County, Conn., deposits in - 186-188

New Jersey, analyses of peat and muck from -..-.-.- 44-45

cultivation of peat soil in_..-- 63-64 deposits In, nature and extent of

limestone in northern, map showing distribution of peat in 


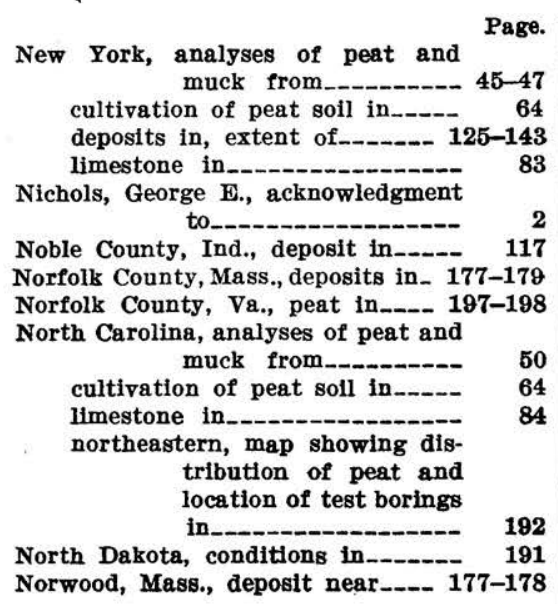

o.

Oak Orchard Swamp, N. Y., description of -._._-_- 133-135

Oakland County, Mich., deposit in - 101-102 Ohio, analyses of peat and muck from.

deposits in, map showing distribution of nature and extent of -.- 120-122 limestone in........ $86,87-88$ Onions, raising of, on peat soil--- 63,64 , Onondaga County, N. Y., deposits $126,137,146$ in

Orange County, N.Y., deposits in.- 138-140 Oregon, occurrence of peat in _...-- 202 Origin of peat._._. 5-7 Orleans County, N. Y., deposits in - 133-135

\section{P.}

Packing material, use of peat as_-Paper, use of peat in Parmelee, C. W., and McCourt, W. E., cited____ 44-45

Parsons, A. L., cited__._. 135

Peat meadow, typical, near Lowell, Mass., plate showing-

Peat moss. See Moss.

Peat swamp, reclaimed, near Big Island, N. Y., plate showing -

Pennsylvania, analyses of peat from deposits in, nature and extent of

limestone in

Perkins, George H., acknowledgment to

Philadelphia County, Pa., deposits inPhysical composition of peat.-.--- 13-16 Pitchy peat, definition of _-_._- 14 Plains, scarcity of peat on Plants, fertilizer, equipment for fertilizer and fuel, location of _ 76-77 operation of
Plants and concerns prepạring peat, distribution of

Plattsburg, N. Y., deposits near-- 126-127 Plymouth County, Mass., deposits

in

Pond, peat-forming, plates showing $\quad 10$

Ponemah, N. H., deposits near--- 152-153

Port Kent, N. Y., deposit near.-- 128-129

Powdered peat, preparation of -.-.- 71

Production of peat from 1916 to

1921 -

Propertles of peat________- 13-59

Providence County, R. I., deposits in 189

Pulaski County, Ind., deposit in -- 117-118

Purpose of the report._._._._- 1-2

\section{Q.}

Quaboag Pond, Mass., deposits near-_._-_. 181-182

R.

Raquette River, N. Y., deposit on- 132-133

Rate of formation of peat

Requa, M. L., Bradley, F. W., and Stalder, W., cited..- 202

Revere Beach, Mass., deposit near_166-167

Rhode Island, analyses of peat and muck from

deposits in, nature and extent of -

Rockingham County, N. H., deposits in, extent of

Rouses Point, N. Y., deposit south of Rutland County, vt., deposits in.--- 165

S.

St. Clair County, Mich., deposit in -- 102 St. Joseph County, Ind., deposits in - 118-119

St. Lawrence County, N. Y., deposits In

Salisbury, vt., deposits near Salt-marsh deposits, examples of _-_ 125 formation of _... 11-12 Scope of the report Seneca County, N. Y., deposit in.- 140-r43 Shaler, N. S., cited_-__-_-_-_ 147-148 Shiawassee County, Mich., deposit in_ 102 Shirley, Mass., deposit in _._._._. 175 Slane, description of plate showing - 62 Soil, peat, cultivation of $126,137,139,140,146$ peat, preparation of _..._._._- 64-65 value of marl on.____- 82 South Carolina, limestone in peat in South Dakota, deposits in_._._._. 91, 191 Sphagnum. See Moss. Spruce-sphagnum bog, plate show-

ing -

Stanton, F. M., analyses by $30-33,34,36,43,50,54-57$

Starke County, Ind., deposits in _.-- 118 Steuben County, Ind., deposits in.- 120 
Page.

Stock feed, use of peat in $4,66,75$

Subsidence, formation of basins by --

Sudbury River, Mass., deposits on- 175-176

Suffolk County, Mass., deposit in - 166-167

Sussex County, N. J., deposit in_- 144-145

Swamp, definition of

Syracuse, N. Y., deposits north of - 135-138

$$
\text { T. }
$$

Tamarack swamp, plate showing--- $\quad 92$ Tazewell County, Ill., deposit in

Tennessee, limestone in

Texas, occurrence of peet in

Texture of peat._._._- 13-15

Topography, influence of, on the formation of peat_..._._. $5,6,8$

Tupper Lake, N. Y., deposit northwest of

Turfy peat, definition of 132-133

\section{D.}

United States, map of, showing distribution of peat deposits and plants for preparing peat.-.- In pocket.

United States Bureau of Mines, analyses supplied by --

$27,34,35,37-43,45,48-49$

Uses of peat........- 2-4, 59-74

\section{V.}

Van Hise, C. R., cited_-_-_-_-- 68

Vegetation, kinds that form peat- 6,14-15 Vermont, analyses of peat and muck from

deposits in, extent of -..- 156-165

Vermont Agricultural Experiment Station, analyses supplied by
Virginia, analyses of peat and muck from.

cultivation of peat soil in

Pagø. deposits in, nature and extent of

limestone in 84

southeastern, map showing distribution of peat and location of test borings in

W.

Wallkill River, N. Y., deposit on-- 138-140

Walpole, Mass., deposit in _._._._ 178

Warren County, N. J., deposit in - 145-146

Washington, occurrence of peat in-- 202

Washington County, R. I., deposits in - 190-191

Washtenaw County, Mich., deposit in_ 102

Water, capacity of peat for

Wayne County, N. Y., deposit in.- 140-143

West Greenville, R. I., deposit near- 189

West Virginia, limestone in_._._. 84

Westerly, R. I., deposits near

White, David, acknowledgment to _-_ 2

Whiteside County, Ill., deposits in - 110-111

Wickham Marsh, near Port Kent, N. Y., description of_ 128-129

Winning of a peat swamp, plates showing -..- 136

Wisconsin, analyses of peat and muck from_-_._- 51-57, 58 deposits in, nature and extent of - 94-96

limestone in... 85

map of, showing distribution of undrained land _...- 94

Wood, peat as a substitute for --_-- 74 Worcester County, Mass., deposits in - 





$$
\text { I19.3:728 }
$$

(MAQ)1 


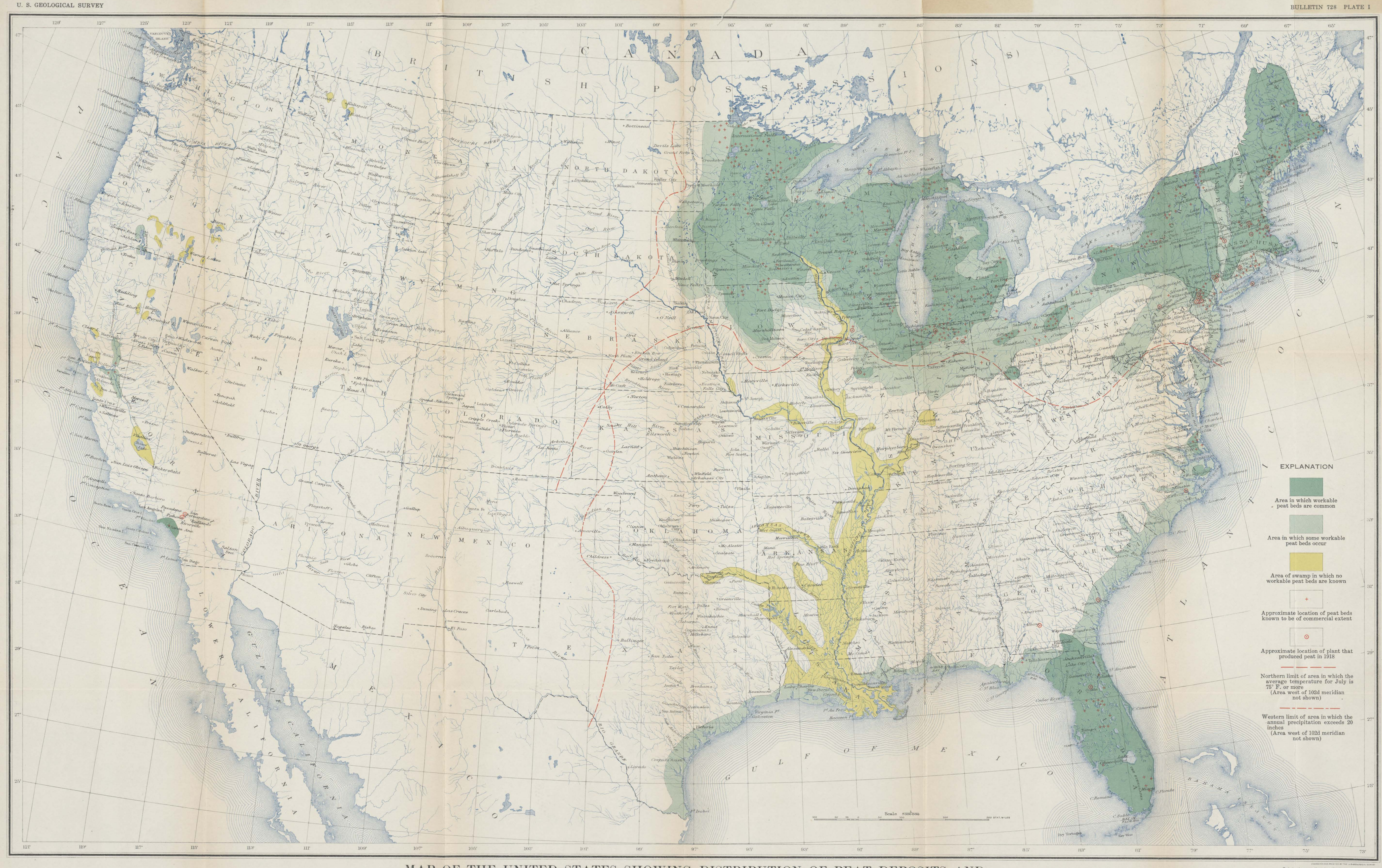




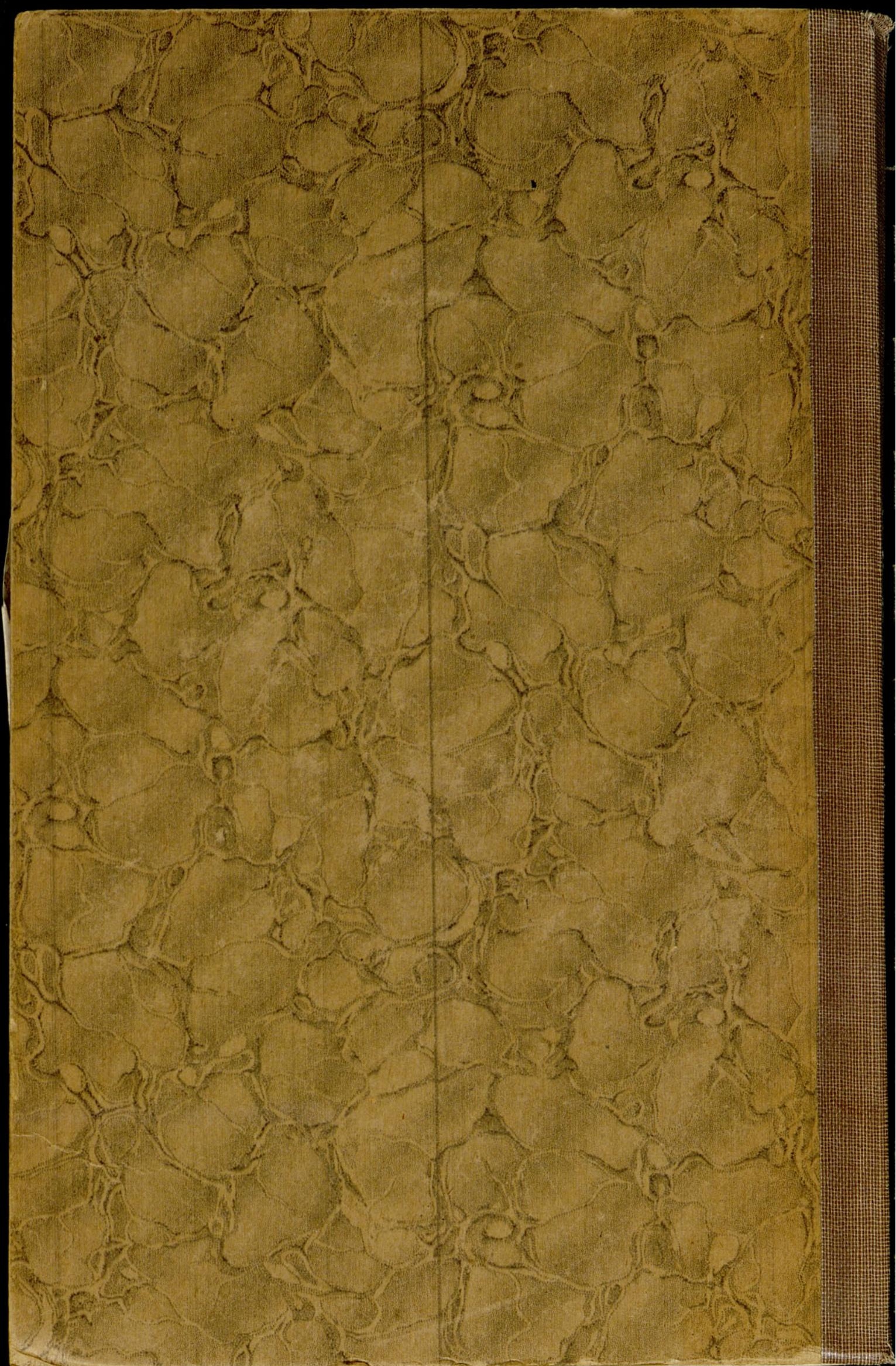

